\title{
Environmentally Friendly Protocol for the Oxidative lodofunctionalization of Olefins in a Green Solvent
}

Wei Yi, Peng-Fei Wang, Meng Lu, Qian-Qian Liu, Xue Bai, Kai-Die Chen, Jing-Wen Zhang and Gong-Qing Liu*

School of Pharmacy, Nantong University, 19 Qixiu Road, Nantong 226001, People's Republic of China

E-mail: gqliu@ntu.edu.cn

\section{Content}

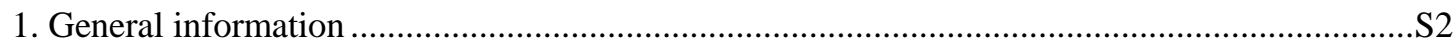

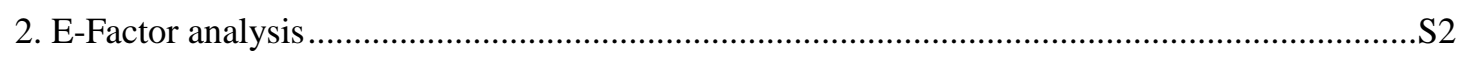

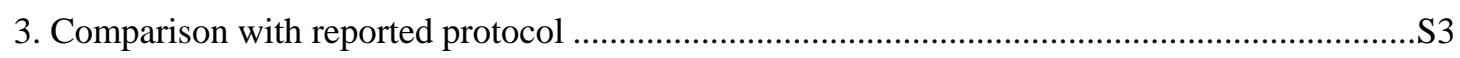

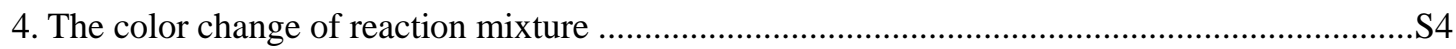

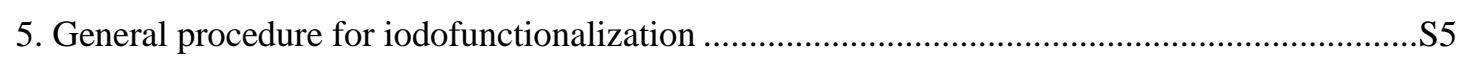

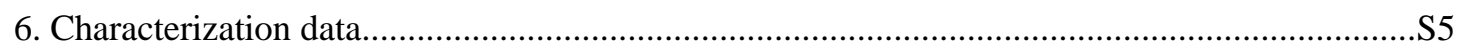

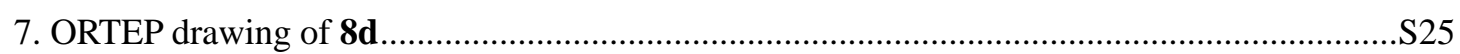

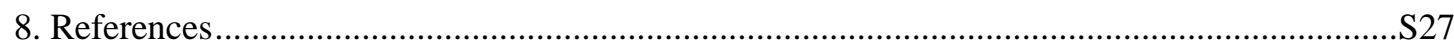

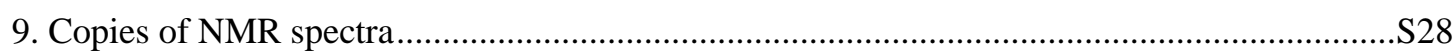

Number of Pages: 96

Number of Figures: 2

Number of Tables: 6 


\section{General information}

Reagents were used as received without further purification unless otherwise indicated. Solvents were dried and distilled prior to use. Reactions were monitored with thin layer chromatography using silica gel $\mathrm{GF}_{254}$ plates. Organic solutions were concentrated in vacuo with a rotavapor. Flash column chromatography was performed using silica gel (200-300 meshes). Petroleum ether used had a boiling point range of $60-90^{\circ} \mathrm{C}$. Melting points were measured on a digital melting point apparatus without correction of the thermometer. Nuclear magnetic resonance spectra were recorded at ambient temperature (unless otherwise stated) at $400 \mathrm{MHz}\left(100 \mathrm{MHz}\right.$ for $\left.{ }^{13} \mathrm{C}\right)$ in $\mathrm{CDCl}_{3}$. Chemical shifts were reported in ppm $(\delta)$ using TMS as internal standard, and spin-spin coupling constants $(J)$ were given in Hz. High resolution mass spectrometry (HRMS) analyses were carried out on an FTICR HR-ESI-MS.

Substrates 1a-1o were prepared by coupling of carboxylic acids and allylamine described by Borhan et al. ${ }^{1} \mathrm{~N}$-alkenyl sulfonamides $\mathbf{3 a - 3} \mathbf{p}^{2}$, N-Boc acrylamide $\mathbf{5 e}^{3}$ and alcohol $\mathbf{5} \mathbf{d}^{3}$ were prepared according to our previous works. Acid 5a and styrene derivatives were purchased from chemical reagent company. Substrate $\mathbf{5 b}$ was synthesized via aldehyde allylation, subsequent Mitsunobu reaction with $\mathrm{N}$-hydroxyphthalimide, N-deprotection and N-sulfonylation according to Chemler's report. ${ }^{4}$ Oxime 5c was prepared from allylbromide and aldehyde. ${ }^{5}$

\section{E-Factor analysis}

According to its original definition (Green Chem. 2007, 9, 1273), the Sheldon E factor value (mass of waste/mass of product) takes into account only the mass of waste generated in a process, and its calculation is performed by simply dividing the sum of the molecular weight of all substances produced by molecular weight of the desired products, with reference to the stoichiometric equation. Thus, the amount of silica gel and the mass of solvent used for work-up and chromatography are usually not included in the calculation. We have followed this trend in our own calculation.

This work:

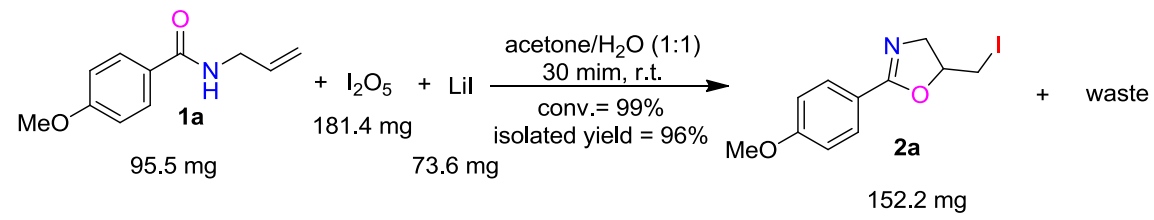

Total amount of reactants: $95.5+181.4+73.6=350.5 \mathrm{mg}$

Amount of final product: $152.2 \mathrm{mg}$

Amount of waste: $350.5-152.2=198.3 \mathrm{mg}$

E-Factor $=$ Amount of waste/Amount of product $=198.3 / 150.6=1.32$ 
Our previous work (

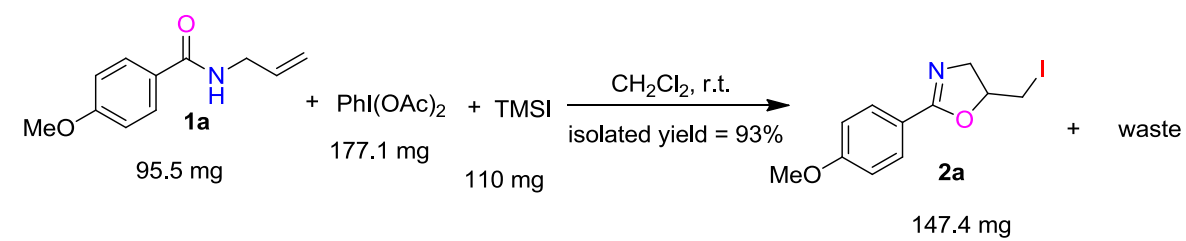

Total amount of reactants: $95.5+171.1+110=376.6 \mathrm{mg}$

Amount of final product: $147.4 \mathrm{mg}$

Amount of waste: $376.6-147.4=229.2 \mathrm{mg}$

E-Factor $=$ Amount of waste/Amount of product $=229.2 / 147.4=1.55$

Krishna's work (Synth. Commun. 2018, 48, 1001-1007):

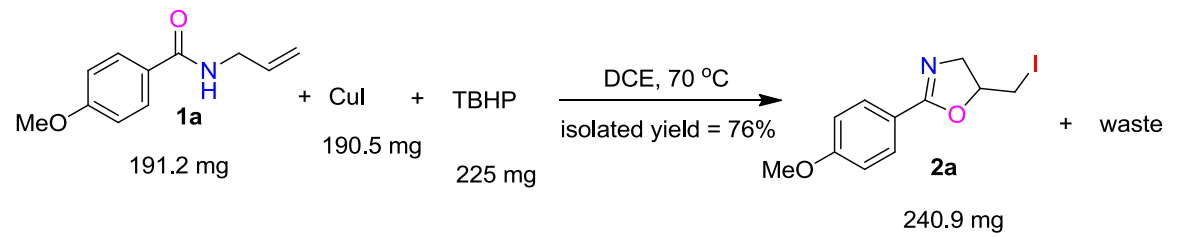

Total amount of reactants: $191.2+190.5+225=606.7 \mathrm{mg}$

Amount of final product: $240.9 \mathrm{mg}$

Amount of waste: $606.7-240.9=365.8 \mathrm{mg}$

E-Factor $=$ Amount of waste/Amount of product $=365.8 / 240.9=1.52$

\section{Comparison with reported protocol}

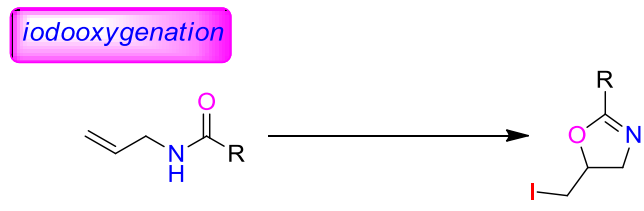

Org. Lett.2006, 3335-3337

This work

$[+]$ transition-metal-free: $t$ - $\mathrm{BuOCl}$,

$[+]$ transition-metal-free: $\mathrm{I}_{2} \mathrm{O}_{5}$,

$[+]$ inorganic iodine source: $\mathrm{NaI}$

[+] inorganic iodine source: LiI

[-] reaction condition: $\mathrm{Ar}$

$[-] \mathrm{CH}_{3} \mathrm{CN}$

[-] one additional chromatographic step

[-] $95 \%$ yield, one substrate
$[+]$ mild reaction condition: air

[+] green solvent: $\mathrm{H}_{2} \mathrm{O}$ /acetone

[+] extraction

[+] 85-96\% yields, 15 substrates

lodoamination

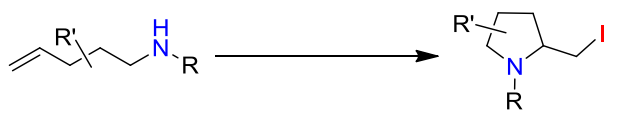

Angew. Chem. Int. Ed. 2012, 51, 3923

[-] transition-metal: $\mathrm{Cu}(\mathrm{OTf})_{2}, \mathrm{MnO}_{2}$,
This work

[+] transition-metal-free: $\mathrm{I}_{2} \mathrm{O}_{5}$, 
[-] organic iodine source: 2-iodopropane

[-] harsh reaction condition: $105{ }^{\circ} \mathrm{C}, \mathrm{Ar}$

[-] $\mathrm{CH}_{3} \mathrm{CN}$

[-] one additional chromatographic step

[-] $15-90 \%$ yields

[+] up to $93 \%$ ee
[+] inorganic iodine source: LiI

[+] mild reaction condition: r.t, air

$[+]$ green solvent: $\mathrm{H}_{2} \mathrm{O}$ /acetone

[+] extraction

[+] 62-93\% yields

[-] NA

iodohydroxylation

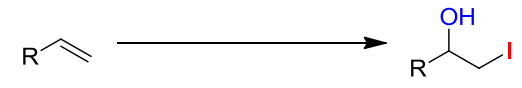

This work

[+] transition-metal-free: $\mathrm{I}_{2} \mathrm{O}_{5}$,

[+] inorganic iodine source: LiI

$[+]$ mild reaction condition: r.t,

$[+]$ green solvent: $\mathrm{H}_{2} \mathrm{O}$ /acetone

[+] extraction

[+] 49-92\% yields

[+] complex organic molecules

\section{iodoetherification}

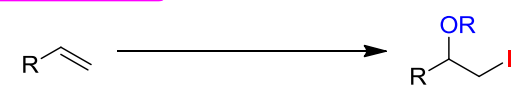

Green Chemistry, 2002, 4, 325-327

This work

[-] transition-metal: EPZ-10 or $\mathrm{ZnCl}_{2}$

$[+]$ transition-metal-free: $\mathrm{I}_{2} \mathrm{O}_{5}$,

[+] iodine source: $\mathrm{I}_{2}$

[+] inorganic iodine source: LiI

[-] reaction condition: reflux under an argon

$[+]$ mild reaction condition: r.t, air

[-] one additional chromatographic step

$[+]$ extraction

\section{The color change of reaction mixture}

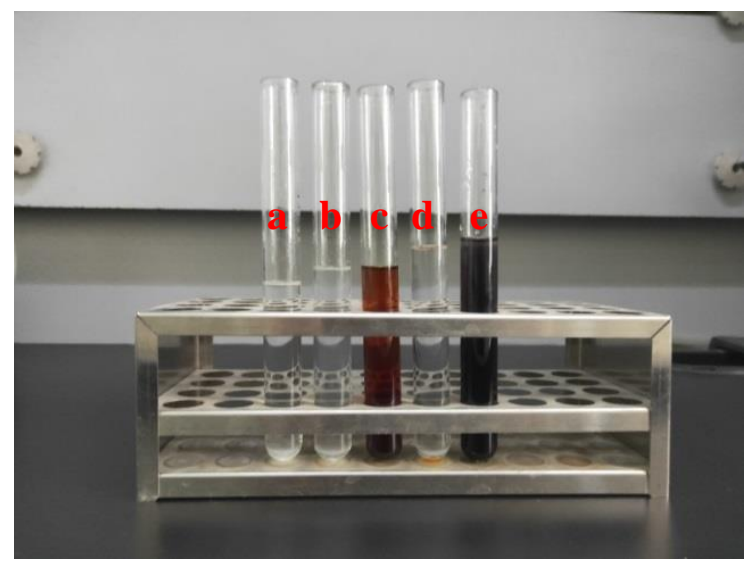

Figure S1.

(a) The acetone/ $\mathrm{H}_{2} \mathrm{O}$ solution with LiI

(b) The acetone/ $\mathrm{H}_{2} \mathrm{O}$ solution with $\mathrm{I}_{2} \mathrm{O}_{5}$ 
(c) The acetone $/ \mathrm{H}_{2} \mathrm{O}$ solution with $\mathrm{LiI}$ and $\mathrm{I}_{2} \mathrm{O}_{5}$

(d) The acetone $/ \mathrm{H}_{2} \mathrm{O}$ solution with $\mathrm{LiI}$ and $\mathrm{I}_{2} \mathrm{O}$ after adding a saturated aqueous $\mathrm{Na}_{2} \mathrm{~S}_{2} \mathrm{O}_{3}$

(e) The acetone/ $\mathrm{H}_{2} \mathrm{O}$ solution with $\mathrm{LiI}$ and $\mathrm{I}_{2} \mathrm{O}$ after adding starch

\section{General procedure for iodofunctionalization}

The reaction was carried out in an open air system. To a $25 \mathrm{~mL}$ flask were added 0.5 mmol substrate, $0.55 \mathrm{mmol}_{2} \mathrm{O}_{5}, 0.55 \mathrm{mmol} \mathrm{LiI}$ and $10 \mathrm{~mL}$ of acetone/water $(1: 1)$. The reaction mixture was stirred at room temperature until complete disappearance of the starting material as shown by TLC ( $c a .0 .5 \mathrm{~h}$ ). The reaction was next quenched with a saturated aqueous $\mathrm{Na}_{2} \mathrm{~S}_{2} \mathrm{O}_{3}$ solution and extracted with EtOAc. The combined organic layers were washed with brine, dried and concentrated to give a crude residue, which was purified by flash column chromatography. In most of the reactions, the products obtained were essentially pure by NMR analysis and further silica gel chromatography was not necessary.

\section{Characterization data}

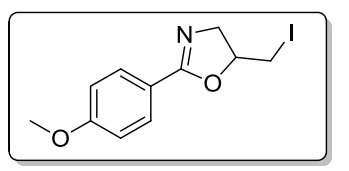

5-(Iodomethyl)-2-(4-methoxyphenyl)-4,5-dihydrooxazole (2a). Compound 2a was prepared according to the general procedure and isolated as a white solid (152 $\mathrm{mg}, 96 \%$ yield) after flash chromatography (petroleum ether/ethyl acetate $=3 / 1$ ). $\mathrm{mp}=$ 104-106 ${ }^{\circ} \mathrm{C} ;{ }^{1} \mathrm{H}$ NMR $\left(400 \mathrm{MHz}, \mathrm{CDCl}_{3}\right) \delta / \mathrm{ppm}=7.80-7.78(\mathrm{~m}, 2 \mathrm{H}), 6.84-6.80(\mathrm{~m}$, 2H), $4.70-4.69(\mathrm{~m}, 1 \mathrm{H}), 4.06(\mathrm{dd}, J=14.9,9.6 \mathrm{~Hz}, 1 \mathrm{H}), 3.71(\mathrm{~s}, 3 \mathrm{H}), 3.68(\mathrm{dd}, J=$ 14.9, $6.3 \mathrm{~Hz}, 1 \mathrm{H}), 3.26-3.20(\mathrm{~m}, 2 \mathrm{H}) .{ }^{13} \mathrm{C} \mathrm{NMR}\left(100 \mathrm{MHz}, \mathrm{CDCl}_{3}\right) \delta / \mathrm{ppm}=163.2$, $162.2,129.9,119.9,113.7,78.2,60.7,55.4,8.0$. Spectral data are in good agreement with literature values. ${ }^{6}$

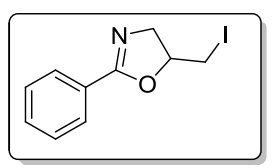

5-(Iodomethyl)-2-phenyl-4,5-dihydrooxazole (2b). Compound 2 b was prepared according to the general procedure and isolated as an oil (135 $\mathrm{mg}, 94 \%$ yield) after a wash with saturated aqueous $\mathrm{Na}_{2} \mathrm{~S}_{2} \mathrm{O}_{3}$ and evaporation. ${ }^{1} \mathrm{H} \mathrm{NMR}\left(400 \mathrm{MHz}, \mathrm{CDCl}_{3}\right.$ ) $\delta / \mathrm{ppm}=7.86-7.84(\mathrm{~m}, 2 \mathrm{H}), 7.40-7.36(\mathrm{~m}, 1 \mathrm{H}), 7.34-7.31(\mathrm{~m}, 2 \mathrm{H}), 4.74-4.63(\mathrm{~m}$, $1 \mathrm{H}), 4.05(\mathrm{dd}, J=15.1,9.6 \mathrm{~Hz}, 1 \mathrm{H}), 3.70(\mathrm{dd}, J=15.1,6.6 \mathrm{~Hz}, 1 \mathrm{H}), 3.27$ (dd, $J=$ $10.3,4.9 \mathrm{~Hz}, 1 \mathrm{H}), 3.21(\mathrm{dd}, J=10.3,7.0 \mathrm{~Hz}, 1 \mathrm{H}) .{ }^{13} \mathrm{C} \mathrm{NMR}\left(100 \mathrm{MHz}, \mathrm{CDCl}_{3}\right)$ $\delta / \mathrm{ppm}=163.5,131.6,128.4,128.2,127.4,78.3,60.7,7.9$. Spectral data are in good agreement with literature values. ${ }^{6}$ 


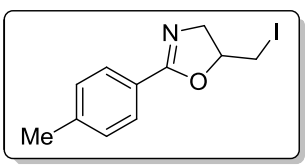

5-(Iodomethyl)-2-(p-tolyl)-4,5-dihydrooxazole (2c). Compound 2c was prepared according to the general procedure and isolated as a white solid (137 mg, 91\% yield) after a wash with saturated aqueous $\mathrm{Na}_{2} \mathrm{~S}_{2} \mathrm{O}_{3}$ and evaporation. $\mathrm{mp}=95-96{ }^{\circ} \mathrm{C} ;{ }^{1} \mathrm{H}$ NMR $\left(400 \mathrm{MHz}, \mathrm{CDCl}_{3}\right) \delta / \mathrm{ppm}=7.75(\mathrm{~d}, J=7.8 \mathrm{~Hz}, 1 \mathrm{H}), 7.14(\mathrm{~d}, J=7.8 \mathrm{~Hz}, 1 \mathrm{H})$, $4.73-4.70(\mathrm{~m}, 1 \mathrm{H}), 4.08(\mathrm{dd}, J=15.1,9.6 \mathrm{~Hz}, 1 \mathrm{H}), 3.71(\mathrm{dd}, J=15.1,6.4 \mathrm{~Hz}, 1 \mathrm{H})$, $3.29-3.23(\mathrm{~m}, 2 \mathrm{H}), 2.31(\mathrm{~s}, 3 \mathrm{H}) .{ }^{13} \mathrm{C}$ NMR $\left(100 \mathrm{MHz}, \mathrm{CDCl}_{3}\right) \delta / \mathrm{ppm}=163.6,141.9$, $129.1,128.2,124.7,78.2,60.7,21.6,7.9$. Spectral data are in good agreement with literature values. ${ }^{6}$

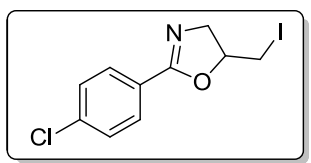

2-(4-Chlorophenyl)-5-(iodomethyl)-4,5-dihydrooxazole (2d). Compound 2d was prepared according to the general procedure and isolated as an oil (147 $\mathrm{mg}, 92 \%$ yield) after a wash with saturated aqueous $\mathrm{Na}_{2} \mathrm{~S}_{2} \mathrm{O}_{3}$ and evaporation. ${ }^{1} \mathrm{H}$ NMR (400 MHz, $\left.\mathrm{CDCl}_{3}\right) \delta / \mathrm{ppm}=7.78(\mathrm{~d}, J=8.5 \mathrm{~Hz}, 1 \mathrm{H}), 7.30(\mathrm{~d}, J=8.5 \mathrm{~Hz}, 1 \mathrm{H}), 4.74-4.71(\mathrm{~m}, 1 \mathrm{H})$, $4.09(\mathrm{dd}, J=14.7,10.3 \mathrm{~Hz}, 1 \mathrm{H}), 3.71(\mathrm{dd}, J=14.7,5.9 \mathrm{~Hz}, 1 \mathrm{H}), 3.36-3.12(\mathrm{~m}, 2 \mathrm{H})$. ${ }^{13} \mathrm{C}$ NMR $\left(100 \mathrm{MHz}, \mathrm{CDCl}_{3}\right) \delta / \mathrm{ppm}=162.7,137.8,129.6,128.7,125.9,78.5,60.7$, 7.7. Spectral data are in good agreement with literature values. ${ }^{6}$

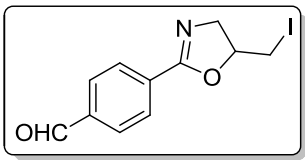

4-(5-(Iodomethyl)-4,5-dihydrooxazol-2-yl)benzaldehyde (2e). Compound 2e was prepared according to the general procedure and isolated as a white solid (140 $\mathrm{mg}, 89 \%$ yield) after a wash with saturated aqueous $\mathrm{Na}_{2} \mathrm{~S}_{2} \mathrm{O}_{3}$ and evaporation. $\mathrm{mp}=62-63{ }^{\circ} \mathrm{C}$; ${ }^{1} \mathrm{H}$ NMR $\left(400 \mathrm{MHz}, \mathrm{CDCl}_{3}\right) \delta / \mathrm{ppm}=10.00(\mathrm{~s}, 1 \mathrm{H}), 8.04-8.02(\mathrm{~m}, 2 \mathrm{H}), 7.87-7.85$ $(\mathrm{m}, 2 \mathrm{H}), 4.86-4.67(\mathrm{~m}, 1 \mathrm{H}), 4.18-4.12(\mathrm{~m}, 1 \mathrm{H}), 3.80-3.75(\mathrm{~m}, 1 \mathrm{H}), 3.36-3.20$ $(\mathrm{m}, 2 \mathrm{H}) .{ }^{13} \mathrm{C} \mathrm{NMR}\left(100 \mathrm{MHz}, \mathrm{CDCl}_{3}\right) \delta / \mathrm{ppm}=191.7,162.6,138.1,132.7,129.6$, $128.8,78.6,61.0,7.6$. Spectral data are in good agreement with literature values. ${ }^{6}$

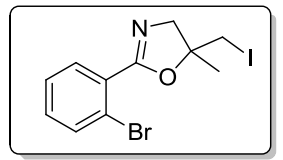

2-(2-Bromophenyl)-5-(iodomethyl)-5-methyl-4,5-dihydrooxazole (2f). Compound 2f was prepared according to the general procedure and isolated as an oil (173 mg, 91\% yield) after a wash with saturated aqueous $\mathrm{Na}_{2} \mathrm{~S}_{2} \mathrm{O}_{3}$ and evaporation. ${ }^{1} \mathrm{H}$ NMR (400 $\left.\mathrm{MHz}, \mathrm{CDCl}_{3}\right) \delta / \mathrm{ppm}=7.63(\mathrm{dd}, J=7.7,1.9 \mathrm{~Hz}, 1 \mathrm{H}), 7.56(\mathrm{dd}, J=7.9,1.3 \mathrm{~Hz}, 1 \mathrm{H})$, $7.28(\mathrm{td}, J=7.5,1.3 \mathrm{~Hz}, 1 \mathrm{H}), 7.25-7.14(\mathrm{~m}, 1 \mathrm{H}), 3.99(\mathrm{~d}, J=15.0 \mathrm{~Hz}, 1 \mathrm{H}), 3.81(\mathrm{~d}$, $J=15.0 \mathrm{~Hz}, 1 \mathrm{H}), 3.43(\mathrm{~d}, J=10.5 \mathrm{~Hz}, 1 \mathrm{H}), 3.36(\mathrm{~d}, J=10.5 \mathrm{~Hz}, 1 \mathrm{H}), 1.64(\mathrm{~s}, 3 \mathrm{H})$. 
${ }^{13} \mathrm{C} \mathrm{NMR}\left(100 \mathrm{MHz}, \mathrm{CDCl}_{3}\right) \delta / \mathrm{ppm}=161.1,132.8,130.7,130.4,128.5,126.1,120.8$, 83.2, 64.8, 24.3, 12.9. HRMS-ESI: calc. for $\left[\mathrm{C}_{11} \mathrm{H}_{11} \mathrm{BrINO}+\mathrm{H}\right]^{+}: \mathrm{m} / \mathrm{z}=379.9147$, found: 379.9139 .

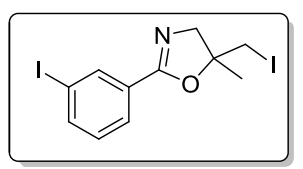

5-(Iodomethyl)-2-(3-iodophenyl)-5-methyl-4,5-dihydrooxazole (2g). Compound 2g was prepared according to the general procedure and isolated as an oil $(188 \mathrm{mg}, 88 \%$ yield) after a wash with saturated aqueous $\mathrm{Na}_{2} \mathrm{~S}_{2} \mathrm{O}_{3}$ and evaporation. ${ }^{1} \mathrm{H}$ NMR (400 $\left.\mathrm{MHz}, \mathrm{CDCl}_{3}\right) \delta / \mathrm{ppm}=8.20(\mathrm{t}, J=1.7 \mathrm{~Hz}, 1 \mathrm{H}), 7.82(\mathrm{dt}, J=7.8,1.3 \mathrm{~Hz}, 1 \mathrm{H}), 7.73(\mathrm{dt}$, $J=8.0,1.4 \mathrm{~Hz}, 1 \mathrm{H}), 7.08(\mathrm{t}, J=7.8 \mathrm{~Hz}, 1 \mathrm{H}), 3.94(\mathrm{~d}, J=15.2 \mathrm{~Hz}, 1 \mathrm{H}), 3.76(\mathrm{~d}, J=$ $15.2 \mathrm{~Hz}, 1 \mathrm{H}), 3.38(\mathrm{~d}, J=10.6 \mathrm{~Hz}, 1 \mathrm{H}), 3.32(\mathrm{~d}, J=10.5 \mathrm{~Hz}, 1 \mathrm{H}), 1.60(\mathrm{~s}, 3 \mathrm{H}) .{ }^{13} \mathrm{C}$ NMR $\left(100 \mathrm{MHz}, \mathrm{CDCl}_{3}\right) \delta / \mathrm{ppm}=160.4,139.2,135.9,129.0,128.6,126.3,92.8,83.1$, 64.5, 24.3, 12.8. HRMS-ESI: calc. for $\left[\mathrm{C}_{11} \mathrm{H}_{11} \mathrm{I}_{2} \mathrm{NO}+\mathrm{H}\right]^{+}: \mathrm{m} / \mathrm{z}=427.9008$, found: 427.9000 .

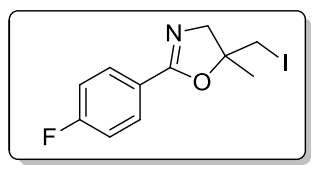

2-(4-Fluorophenyl)-5-(iodomethyl)-5-methyl-4,5-dihydrooxazole (2h). Compound $\mathbf{2 h}$ was prepared according to the general procedure and isolated as an oil (145 mg, 91\% yield) after a wash with saturated aqueous $\mathrm{Na}_{2} \mathrm{~S}_{2} \mathrm{O}_{3}$ and evaporation. ${ }^{1} \mathrm{H}$ NMR (400 $\left.\mathrm{MHz}, \mathrm{CDCl}_{3}\right) \delta / \mathrm{ppm}=7.99-7.77(\mathrm{~m}, 2 \mathrm{H}), 7.14-6.93(\mathrm{~m}, 2 \mathrm{H}), 3.94(\mathrm{~d}, J=15.0 \mathrm{~Hz}$, $1 \mathrm{H}), 3.77(\mathrm{~d}, J=15.0 \mathrm{~Hz}, 1 \mathrm{H}), 3.39$ (d, $J=10.5 \mathrm{~Hz}, 1 \mathrm{H}), 3.34(\mathrm{~d}, J=10.5 \mathrm{~Hz}, 1 \mathrm{H})$, 1.61 (s, 3H). ${ }^{19} \mathrm{~F}$ NMR $\left(376 \mathrm{MHz}, \mathrm{CDCl}_{3}\right) \delta / \mathrm{ppm}=-107.82 .{ }^{13} \mathrm{C} \mathrm{NMR}(100 \mathrm{MHz}$, $\left.\mathrm{CDCl}_{3}\right) \delta / \mathrm{ppm}=165.0,162.5,161.1,129.4(\mathrm{~d}, J=8.8 \mathrm{~Hz}), 114.5(\mathrm{~d}, J=22.0 \mathrm{~Hz})$, 83.1, 64.4, 24.3, 12.9. HRMS-ESI: calc. for $\left[\mathrm{C}_{11} \mathrm{H}_{11} \mathrm{FINO}+\mathrm{H}\right]^{+}: \mathrm{m} / \mathrm{z}=319.9948$, found: 319.9935 .

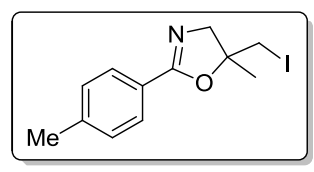

5-(Iodomethyl)-5-methyl-2-(p-tolyl)-4,5-dihydrooxazole (2i). Compound $2 \mathbf{i}$ was prepared according to the general procedure and isolated as an oil (143 $\mathrm{mg}, 91 \%$ yield) after a wash with saturated aqueous $\mathrm{Na}_{2} \mathrm{~S}_{2} \mathrm{O}_{3}$ and evaporation. ${ }^{1} \mathrm{H}$ NMR (400 MHz, $\left.\mathrm{CDCl}_{3}\right) \delta / \mathrm{ppm}=7.84(\mathrm{~d}, J=8.0 \mathrm{~Hz}, 2 \mathrm{H}), 7.24(\mathrm{~d}, J=8.0 \mathrm{~Hz}, 2 \mathrm{H}), 4.02(\mathrm{~d}, J=15.0$ $\mathrm{Hz}, 1 \mathrm{H}), 3.84(\mathrm{~d}, J=15.0 \mathrm{~Hz}, 1 \mathrm{H}), 3.47(\mathrm{~d}, J=10.5 \mathrm{~Hz}, 1 \mathrm{H}), 3.43(\mathrm{~d}, J=10.5 \mathrm{~Hz}$, $1 \mathrm{H}), 2.41(\mathrm{~s}, 3 \mathrm{H}), 1.69(\mathrm{~s}, 3 \mathrm{H}) .{ }^{13} \mathrm{C} \mathrm{NMR}\left(100 \mathrm{MHz}, \mathrm{CDCl}_{3}\right) \delta / \mathrm{ppm}=163.0,141.8$, 129.1, 128.1, 125.0, 83.6, 65.5, 25.3, 21.59, 14.1. HRMS-ESI: calc. for $\left[\mathrm{C}_{12} \mathrm{H}_{14} \mathrm{INO}+\mathrm{H}\right]^{+}: \mathrm{m} / \mathrm{z}=316.0198$, found: 316.0188 . 


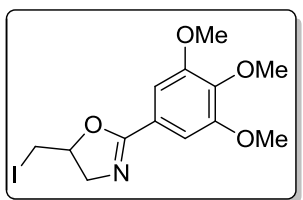

5-(Iodomethyl)-2-(3,4,5-trimethoxyphenyl)-4,5-dihydrooxazole (2j). Compound $2 \mathbf{j}$ was prepared according to the general procedure and isolated as an oil (162 mg, 86\% yield) after flash chromatography (petroleum ether/ethyl acetate $=7 / 1) .{ }^{1} \mathrm{H}$ NMR $(400$ $\left.\mathrm{MHz}, \mathrm{CDCl}_{3}\right) \delta / \mathrm{ppm}=7.20(\mathrm{~s}, 1 \mathrm{H}), 7.19(\mathrm{~s}, 1 \mathrm{H}), 4.86-4.78(\mathrm{~m}, 2 \mathrm{H}) 4.19(\mathrm{dd}, J=$ 15.2, 9.5, Hz, 1H), 3.90 (s, 3H), 3.89 (s, 3H), 3.88 (s, 3H), 3.82 (dd, $J=15.1,6.6 \mathrm{~Hz}$, $1 \mathrm{H}), 3.41(\mathrm{dd}, J=10.2,4.6 \mathrm{~Hz}, 1 \mathrm{H}), 3.35(\mathrm{dd}, J=10.1,7.1 \mathrm{~Hz}, 1 \mathrm{H}) .{ }^{13} \mathrm{C}$ NMR $(100$ $\left.\mathrm{MHz}, \mathrm{CDCl}_{3}\right) \delta / \mathrm{ppm}=163.4,153.0,140.9,122.6,105.3,78.4,61.0,60.9,60.8,56.2$, 7.8. HRMS-ESI: calc. for $\left[\mathrm{C}_{13} \mathrm{H}_{16} \mathrm{INO}_{4}+\mathrm{H}\right]^{+}: \mathrm{m} / \mathrm{z}=378.0202$, found: 378.0210 .

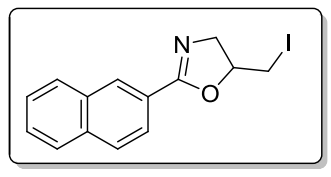

5-(Iodomethyl)-2-(naphthalen-2-yl)-4,5-dihydrooxazole (2k). Compound 2k was prepared according to the general procedure and isolated as a white solid (148 $\mathrm{mg}, 88 \%$ yield) after flash chromatography (petroleum ether/ethyl acetate $=4 / 1$ ) $. \mathrm{mp}=$ 100-101 ${ }^{\circ} \mathrm{C} ;{ }^{1} \mathrm{H}$ NMR (400 MHz, $\mathrm{CDCl}_{3}$ ) $\delta / \mathrm{ppm}=8.32$ (s, 1H), 7.91 (dd, $J=8.2,1.4$ $\mathrm{Hz}, 1 \mathrm{H}), 7.84-7.77(\mathrm{~m}, 1 \mathrm{H}), 7.73(\mathrm{t}, J=8.2 \mathrm{~Hz}, 2 \mathrm{H}), 7.49-7.33(\mathrm{~m}, 2 \mathrm{H}), 4.76-$ $4.62(\mathrm{~m}, 1 \mathrm{H}), 4.09(\mathrm{dd}, J=15.2,9.5 \mathrm{~Hz}, 1 \mathrm{H}), 3.72(\mathrm{dd}, J=15.2,6.6 \mathrm{~Hz}, 1 \mathrm{H}), 3.29-$ 3.19 (m, 2H). ${ }^{13} \mathrm{C}$ NMR $\left(100 \mathrm{MHz}, \mathrm{CDCl}_{3}\right) \delta / \mathrm{ppm}=163.6,134.7,132.6,128.9,128.8$, $128.2,127.8,127.6,126.6,124.8,124.7,78.4,60.9,7.9$. Spectral data are in good agreement with literature values. ${ }^{6}$

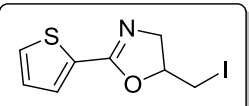

5-(Iodomethyl)-2-(thiophen-2-yl)-4,5-dihydrooxazole (2l). Compound 2l was prepared according to the general procedure and isolated as an oil (132 $\mathrm{mg}, 90 \%$ yield) after a wash with saturated aqueous $\mathrm{Na}_{2} \mathrm{~S}_{2} \mathrm{O}_{3}$ and evaporation. ${ }^{1} \mathrm{H}$ NMR (400 MHz, $\left.\mathrm{CDCl}_{3}\right) \delta / \mathrm{ppm}=7.54(\mathrm{dd}, J=3.7,1.2 \mathrm{~Hz}, 1 \mathrm{H}), 7.40(\mathrm{dd}, J=5.0,1.3 \mathrm{~Hz}, 1 \mathrm{H}), 7.01(\mathrm{dd}$, $J=5.0,3.7 \mathrm{~Hz}, 1 \mathrm{H}), 4.78-4.71(\mathrm{~m}, 1 \mathrm{H}), 4.09(\mathrm{dd}, J=15.0,9.4 \mathrm{~Hz}, 1 \mathrm{H}), 3.71(\mathrm{dd}, J=$ 15.0, $6.6 \mathrm{~Hz}, 1 \mathrm{H}), 3.31(\mathrm{dd}, J=10.3,4.8 \mathrm{~Hz}, 1 \mathrm{H}), 3.25(\mathrm{dd}, J=10.3,7.1 \mathrm{~Hz}, 1 \mathrm{H}) .{ }^{13} \mathrm{C}$ NMR (100 MHz, $\left.\mathrm{CDCl}_{3}\right) \delta / \mathrm{ppm}=158.3,129.5,129.1,129.0,126.6,77.8,59.7,6.2$. Spectral data are in good agreement with literature values. ${ }^{7}$

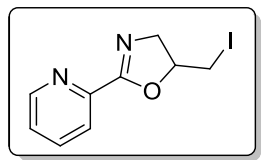

5-(Iodomethyl)-2-(pyridin-2-yl)-4,5-dihydrooxazole (2m). Compound $2 \mathrm{~m}$ was prepared according to the general procedure and isolated as a white solid (127 $\mathrm{mg}, 88 \%$ yield) after a wash with saturated aqueous $\mathrm{Na}_{2} \mathrm{~S}_{2} \mathrm{O}_{3}$ and evaporation. ${ }^{1} \mathrm{H}$ NMR (400 
$\left.\mathrm{MHz}, \mathrm{CDCl}_{3}\right) \delta / \mathrm{ppm}=8.63-8.62(\mathrm{~m}, 1 \mathrm{H}), 7.96-7.94(\mathrm{~m}, 1 \mathrm{H}), 7.720-7.68(\mathrm{~m}, 1 \mathrm{H})$, $7.33-7.30(\mathrm{~m}, 1 \mathrm{H}), 4.86-4.75(\mathrm{~m}, 1 \mathrm{H}), 4.17(\mathrm{dd}, J=15.6,9.6 \mathrm{~Hz}, 1 \mathrm{H}), 3.79$ (dd, $J$ $=15.6,7.0 \mathrm{~Hz}, 1 \mathrm{H}), 3.38(\mathrm{dd}, J=10.1,4.1 \mathrm{~Hz}, 1 \mathrm{H}), 3.29(\mathrm{dd}, J=10.1,7.7 \mathrm{~Hz}, 1 \mathrm{H})$. ${ }^{13} \mathrm{C} \mathrm{NMR}\left(100 \mathrm{MHz}, \mathrm{CDCl}_{3}\right) \delta / \mathrm{ppm}=162.6,149.7,146.3,136.7,125.9,123.9,79.0$, $61.0,7.8$. Spectral data are in good agreement with literature values. ${ }^{6}$

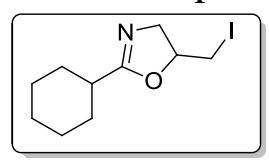

2-Cyclohexyl-5-(iodomethyl)-4,5-dihydrooxazole (2n). Compound 2n was prepared according to the general procedure and isolated as an oil (118 $\mathrm{mg}, 81 \%$ yield) after flash chromatography (petroleum ether/ethyl acetate $=2 / 1) .{ }^{1} \mathrm{H}$ NMR $(400 \mathrm{MHz}$, $\left.\mathrm{CDCl}_{3}\right) \delta / \mathrm{ppm}=4.57-4.34(\mathrm{~m}, 1 \mathrm{H}), 3.86(\mathrm{dd}, J=14.2,9.8 \mathrm{~Hz}, 1 \mathrm{H}), 3.49(\mathrm{dd}, J=$ 14.2, $6.2 \mathrm{~Hz}, 1 \mathrm{H}), 3.19-3.17(\mathrm{~m}, 2 \mathrm{H}), 2.22(\mathrm{t}, J=11.1 \mathrm{~Hz}, 1 \mathrm{H}), 1.87$ (s, 2H), $1.72-$ $1.69(\mathrm{~m}, 2 \mathrm{H}), 1.60(\mathrm{~d}, J=7.8 \mathrm{~Hz}, 1 \mathrm{H}), 1.46-1.27(\mathrm{~m}, 2 \mathrm{H}), 1.30-1.12(\mathrm{~m}, 3 \mathrm{H}) .{ }^{13} \mathrm{C}$ NMR $\left(100 \mathrm{MHz}, \mathrm{CDCl}_{3}\right) \delta / \mathrm{ppm}=170.7,77.2,60.0,37.4,29.8,29.7,25.8,25.6,25.6$, 8.5. 8. Spectral data are in good agreement with literature values. ${ }^{6}$

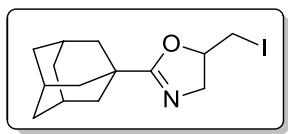

5-(Iodomethyl)-2-adamantyl-4,5-dihydrooxazole (2o). Compound 20 was prepared according to the general procedure and isolated as an oil (147 $\mathrm{mg}, 85 \%$ yield) after flash chromatography (petroleum ether/ethyl acetate $=2 / 1) .{ }^{1} \mathrm{H}$ NMR (400 MHz, $\left.\mathrm{CDCl}_{3}\right) \delta / \mathrm{ppm}=4.47-4.40(\mathrm{~m}, 1 \mathrm{H}), 3.86-3.82(\mathrm{~m}, 1 \mathrm{H}), 3.62-3.39(\mathrm{~m}, 1 \mathrm{H}), 3.17(\mathrm{t}$, $J=4.9 \mathrm{~Hz}, 2 \mathrm{H}), 1.92-1.90(\mathrm{~m}, 3 \mathrm{H}), 1.82(\mathrm{~s}, 6 \mathrm{H}), 1.65(\mathrm{~s}, 6 \mathrm{H}) .{ }^{13} \mathrm{C} \mathrm{NMR}(100 \mathrm{MHz}$, $\left.\mathrm{CDCl}_{3}\right) \delta / \mathrm{ppm}=173.2,76.9,59.8,39.4,36.5,35.3,27.8,8.6$. Spectral data are in good agreement with literature values. ${ }^{6}$

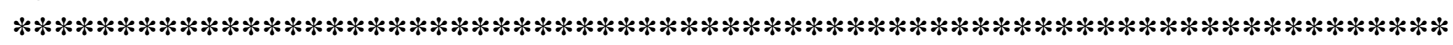

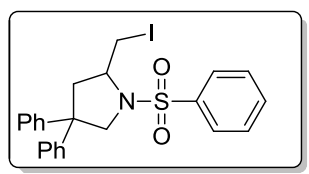

2-(Iodomethyl)-4,4-diphenyl-1-(phenylsulfonyl)pyrrolidine (4a). Compound 4a was prepared according to the general procedure and isolated as a white solid (226 mg, $90 \%$ yield) after a wash with saturated aqueous $\mathrm{Na}_{2} \mathrm{~S}_{2} \mathrm{O}_{3}$ and evaporation. mp $=$ 153-154 ${ }^{\circ} \mathrm{C} ;{ }^{1} \mathrm{H}$ NMR $\left(400 \mathrm{MHz}, \mathrm{CDCl}_{3}\right) \delta / \mathrm{ppm}=7.71-7.60(\mathrm{~m}, 2 \mathrm{H}), 7.45(\mathrm{t}, J=7.5$ $\mathrm{Hz}, 1 \mathrm{H}), 7.36-7.32(\mathrm{~m}, 2 \mathrm{H}), 7.23-7.15(\mathrm{~m}, 4 \mathrm{H}), 7.14-7.00(\mathrm{~m}, 6 \mathrm{H}), 4.35(\mathrm{~d}, J=10.3$ $\mathrm{Hz}, 1 \mathrm{H}), 3.85-3.77(\mathrm{~m}, 1 \mathrm{H}), 3.74(\mathrm{~d}, J=10.3 \mathrm{~Hz}, 1 \mathrm{H}), 3.59$ (dd, $J=9.6,3.0 \mathrm{~Hz}, 1 \mathrm{H})$, 2.82-2.69 (m, 2H), $2.60(\mathrm{dd}, J=13.1,5.3 \mathrm{~Hz}, 1 \mathrm{H}) ;{ }^{13} \mathrm{C}$ NMR $\left(100 \mathrm{MHz}, \mathrm{CDCl}_{3}\right)$ $\delta / \mathrm{ppm}=143.5,143.2,136.1,131.8,128.1,127.7,127.6,126.2,125.8,125.7,125.0$, $125.2,59.4,58.1,51.1,42.8,10.3$. Spectral data are in good agreement with literature values. $^{8}$ 


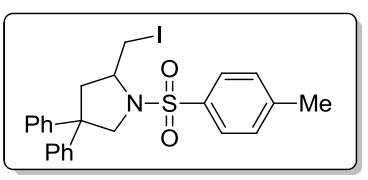

2-(Iodomethyl)-4,4-diphenyl-1-tosylpyrrolidine (4b). Compound 4b was prepared according to the general procedure and isolated as a white solid $(230 \mathrm{mg}, 89 \%$ yield) after a wash with saturated aqueous $\mathrm{Na}_{2} \mathrm{~S}_{2} \mathrm{O}_{3}$ and evaporation. $\mathrm{mp}=180-182{ }^{\circ} \mathrm{C} ;{ }^{1} \mathrm{H}$ NMR $\left(400 \mathrm{MHz}, \mathrm{CDCl}_{3}\right) \delta / \mathrm{ppm}=7.50(\mathrm{~d}, J=8.1 \mathrm{~Hz}, 2 \mathrm{H}), 7.18-7.17(\mathrm{~m}, 4 \mathrm{H})$, 7.10-6.96 (m, 8H), $4.35(\mathrm{~d}, J=10.3 \mathrm{~Hz}, 1 \mathrm{H}), 3.82-3.72(\mathrm{~m}, 1 \mathrm{H}), 3.67(\mathrm{~d}, J=10.3 \mathrm{~Hz}$, $1 \mathrm{H}), 3.59$ (dd, $J=9.5,2.9 \mathrm{~Hz}, 1 \mathrm{H}), 2.77-2.71(\mathrm{~m}, 2 \mathrm{H}), 2.56(\mathrm{dd}, J=13.1,5.2 \mathrm{~Hz}, 1 \mathrm{H})$, 2.29 (s, 3H); ${ }^{13} \mathrm{C} \mathrm{NMR}\left(100 \mathrm{MHz}, \mathrm{CDCl}_{3}\right) \delta / \mathrm{ppm}=143.6,143.3,142.6,132.8,128.7$, 127.7, 127.6, 126.3, 125.7, 125.5, 125.4, 125.2, 59.3, 58.1, 51.1, 42.7, 20.5, 10.5. Spectral data are in good agreement with literature values. ${ }^{8}$

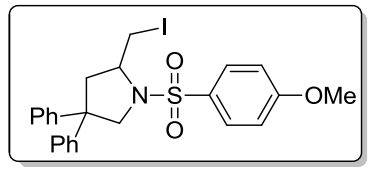

2-(Iodomethyl)-1-((4-methoxyphenyl)sulfonyl)-4,4-diphenylpyrrolidine

(4c).

Compound $\mathbf{4} \mathbf{c}$ was prepared according to the general procedure and isolated as a yellow solid (226 mg, 85\% yield) after flash chromatography (petroleum ether/ethyl acetate $=10 / 1) . \mathrm{mp}=163-165{ }^{\circ} \mathrm{C} .{ }^{1} \mathrm{H} \mathrm{NMR}\left(400 \mathrm{MHz}, \mathrm{CDCl}_{3}\right) \delta / \mathrm{ppm}=7.57(\mathrm{~d}, J=$ $8.9 \mathrm{~Hz}, 2 \mathrm{H}), 7.19$ (q, $J=3.2 \mathrm{~Hz}, 4 \mathrm{H}), 7.07$ (ddt, $J=12.0,8.4,4.6 \mathrm{~Hz}, 6 \mathrm{H}), 6.79$ (d, $J$ $=8.9 \mathrm{~Hz}, 2 \mathrm{H}), 4.36(\mathrm{~d}, J=10.2 \mathrm{~Hz}, 1 \mathrm{H}), 3.82-3.74(\mathrm{~m}, 4 \mathrm{H}), 3.67(\mathrm{~d}, J=10.3 \mathrm{~Hz}$, $1 \mathrm{H}), 3.59(\mathrm{dd}, J=9.6,3.1 \mathrm{~Hz}, 1 \mathrm{H}), 2.81-2.70(\mathrm{~m}, 2 \mathrm{H}), 2.58(\mathrm{dd}, J=13.1,5.2 \mathrm{~Hz}$, $1 \mathrm{H}) .{ }^{13} \mathrm{C} \mathrm{NMR}\left(100 \mathrm{MHz}, \mathrm{CDCl}_{3}\right) \delta / \mathrm{ppm}=161.9,143.7,143.4,128.4,127.6(\mathrm{t}, J=$ $4.5 \mathrm{~Hz}$ ), 125.8, 125.6 (d, $J=1.8 \mathrm{~Hz}), 125.3,113.3,59.4,58.2,54.6,51.2,42.8,10.5$. ESI-HRMS: calc. for $\left[\mathrm{C}_{24} \mathrm{H}_{24} \mathrm{INO}_{3} \mathrm{~S}+\mathrm{H}\right]^{+}: \mathrm{m} / \mathrm{z}=534.0600$, found: 534.0638 .

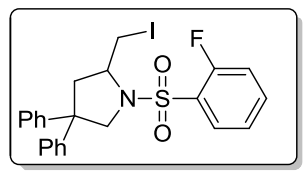

1-((2-Fluorophenyl)sulfonyl)-2-(iodomethyl)-4,4-diphenylpyrrolidine

(4d).

Compound $\mathbf{4 d}$ was prepared according to the general procedure and isolated as a yellow solid (242 mg, 93\% yield) after a wash with saturated aqueous $\mathrm{Na}_{2} \mathrm{~S}_{2} \mathrm{O}_{3}$ and evaporation. $\mathrm{mp}=112-114{ }^{\circ} \mathrm{C} .{ }^{1} \mathrm{H}$ NMR $\left(400 \mathrm{MHz}, \mathrm{CDCl}_{3}\right) \delta / \mathrm{ppm}=7.77-7.71(\mathrm{~m}$, 1H), $7.42-7.34(\mathrm{~m}, 1 \mathrm{H}), 7.22-7.17(\mathrm{~m}, 2 \mathrm{H}), 7.15-7.04(\mathrm{~m}, 8 \mathrm{H}), 7.01(\mathrm{t}, J=6.9 \mathrm{~Hz}$, $1 \mathrm{H}), 6.96-6.87(\mathrm{~m}, 1 \mathrm{H}), 4.18(\mathrm{dd}, J=25.3,10.8 \mathrm{~Hz}, 2 \mathrm{H}), 3.94(\mathrm{dd}, J=13.0,7.3 \mathrm{~Hz}$, $1 \mathrm{H}), 3.51(\mathrm{dd}, J=9.9,2.8 \mathrm{~Hz}, 1 \mathrm{H}), 3.07-3.00(\mathrm{~m}, 1 \mathrm{H}), 2.96(\mathrm{dd}, J=13.0,7.0 \mathrm{~Hz}$, $1 \mathrm{H}), 2.52(\mathrm{dd}, J=13.0,7.6 \mathrm{~Hz}, 1 \mathrm{H}) .{ }^{13} \mathrm{C} \mathrm{NMR}\left(100 \mathrm{MHz}, \mathrm{CDCl}_{3}\right) \delta / \mathrm{ppm}=159.0$, 156.5, 143.6, 142.7, $133.8(\mathrm{~d}, J=8.5 \mathrm{~Hz}), 129.3,127.6(\mathrm{~d}, J=7.1 \mathrm{~Hz}), 125.8(\mathrm{~d}, J=$ $5.5 \mathrm{~Hz}), 125.5,125.3,123.3(\mathrm{~d}, J=3.7 \mathrm{~Hz}), 116.5,116.2,58.6,57.8,51.5,43.6,10.7$. ESI-HRMS: calc. for $\left[\mathrm{C}_{23} \mathrm{H}_{21} \mathrm{FINO}_{2} \mathrm{~S}+\mathrm{H}\right]^{+}: \mathrm{m} / \mathrm{z}=522.0400$, found: 522.0435 . 


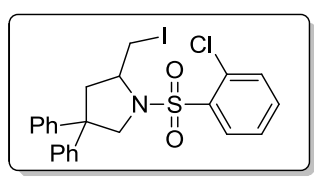

1-((2-Chlorophenyl)sulfonyl)-2-(iodomethyl)-4,4-diphenylpyrrolidine

(4e).

Compound 4e was prepared according to the general procedure and isolated as a yellow solid (236 mg, 88\% yield) after a wash with saturated aqueous $\mathrm{Na}_{2} \mathrm{~S}_{2} \mathrm{O}_{3}$ and evaporation. $\mathrm{mp}=147-149{ }^{\circ} \mathrm{C} .{ }^{1} \mathrm{H}$ NMR $\left(400 \mathrm{MHz}, \mathrm{CDCl}_{3}\right) \delta / \mathrm{ppm}=8.03(\mathrm{~d}, J=8.3$ $\mathrm{Hz}, 1 \mathrm{H}), 7.46-7.41(\mathrm{~m}, 2 \mathrm{H}), 7.38-7.33(\mathrm{~m}, 1 \mathrm{H}), 7.32-7.26(\mathrm{~m}, 4 \mathrm{H}), 7.26-7.20$ $(\mathrm{m}, 5 \mathrm{H}), 7.16(\mathrm{dd}, J=13.3,6.2 \mathrm{~Hz}, 1 \mathrm{H}), 4.60(\mathrm{~d}, J=10.8 \mathrm{~Hz}, 1 \mathrm{H}), 4.12(\mathrm{~d}, J=10.8$ $\mathrm{Hz}, 1 \mathrm{H}), 4.01(\mathrm{~d}, J=6.6 \mathrm{~Hz}, 1 \mathrm{H}), 3.42(\mathrm{~d}, J=10.2 \mathrm{~Hz}, 1 \mathrm{H}), 3.06(\mathrm{dd}, J=10.1,7.8 \mathrm{~Hz}$, $1 \mathrm{H}), 2.98(\mathrm{dd}, J=11.1,6.7 \mathrm{~Hz}, 1 \mathrm{H}), 2.64(\mathrm{dd}, J=12.7,8.4 \mathrm{~Hz}, 1 \mathrm{H}) .{ }^{13} \mathrm{C}$ NMR $(100$ $\left.\mathrm{MHz}, \mathrm{CDCl}_{3}\right) \delta / \mathrm{ppm}=144.6,143.7,138.5,133.6(\mathrm{~d}, J=2.9 \mathrm{~Hz}), 132.6,132.2,130.9$, $128.8(\mathrm{~d}, J=1.6 \mathrm{~Hz}), 127.1(\mathrm{~d}, J=2.3 \mathrm{~Hz}), 126.8(\mathrm{~d}, J=4.5 \mathrm{~Hz}), 126.6,126.5,59.6$, 59.4, 52.6, 45.2, 11.4. ESI-HRMS: calc. for $\left[\mathrm{C}_{23} \mathrm{H}_{21} \mathrm{ClINO}_{2} \mathrm{~S}+\mathrm{H}\right]^{+}: \mathrm{m} / \mathrm{z}=538.0104$, found: 538.0100 .

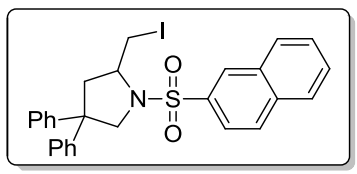

2-(Iodomethyl)-1-(naphthalen-2-ylsulfonyl)-4,4-diphenylpyrrolidine

(4f).

Compound $\mathbf{4 f}$ was prepared according to the general procedure and isolated as a yellow solid (235 mg, 85\% yield) after flash chromatography (petroleum ether/ethyl acetate $=12 / 1) . \mathrm{mp}=146-148{ }^{\circ} \mathrm{C} .{ }^{1} \mathrm{H}$ NMR $\left(400 \mathrm{MHz}, \mathrm{CDCl}_{3}\right) \delta / \mathrm{ppm}=8.21(\mathrm{~s}, 1 \mathrm{H})$, $7.83(\mathrm{dd}, J=20.3,8.0 \mathrm{~Hz}, 2 \mathrm{H}), 7.73(\mathrm{~d}, J=8.7 \mathrm{~Hz}, 1 \mathrm{H}), 7.59-7.53(\mathrm{~m}, 2 \mathrm{H}), 7.23-$ $7.07(\mathrm{~m}, 6 \mathrm{H}), 6.94(\mathrm{~d}, J=7.4 \mathrm{~Hz}, 2 \mathrm{H}), 6.85(\mathrm{t}, J=7.6 \mathrm{~Hz}, 2 \mathrm{H}), 6.77(\mathrm{t}, J=7.2 \mathrm{~Hz}$, $1 \mathrm{H}), 4.37(\mathrm{~d}, J=10.5 \mathrm{~Hz}, 1 \mathrm{H}), 3.89(\mathrm{t}, J=9.3 \mathrm{~Hz}, 2 \mathrm{H}), 3.68(\mathrm{dd}, J=9.6,3.0 \mathrm{~Hz}, 1 \mathrm{H})$, $2.94-2.79(\mathrm{~m}, 2 \mathrm{H}), 2.53(\mathrm{dd}, J=13.2,6.0 \mathrm{~Hz}, 1 \mathrm{H}) .{ }^{13} \mathrm{C} \mathrm{NMR}\left(100 \mathrm{MHz}, \mathrm{CDCl}_{3}\right)$ $\delta / \mathrm{ppm}=143.7,142.9,133.9,133.3,131.1,128.4,128.6,127.8,127.7,127.6,127.4$, $126.8,126.4,125.8,125.5,125.4,125.1,121.5,59.36,58.4,51.4,43.1,10.5$. ESI-HRMS: calc. for $\left[\mathrm{C}_{27} \mathrm{H}_{24} \mathrm{INO}_{2} \mathrm{~S}+\mathrm{H}\right]^{+}: \mathrm{m} / \mathrm{z}=554.0651$, found: 554.0629 .

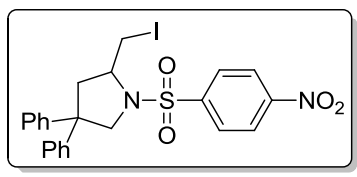

2-(Iodomethyl)-1-((4-nitrophenyl)sulfonyl)-4,4-diphenylpyrrolidine

(4g).

Compound $\mathbf{4 g}$ was prepared according to the general procedure and isolated as a yellow solid (170 mg, 62\% yield) after flash chromatography (petroleum ether/ethyl acetate $=40 / 1)$. H NMR $\left(400 \mathrm{MHz}, \mathrm{CDCl}_{3}\right) \delta / \mathrm{ppm}=8.02(\mathrm{~d}, J=8.9 \mathrm{~Hz}, 2 \mathrm{H}), 7.66(\mathrm{~d}$, $J=8.9 \mathrm{~Hz}, 2 \mathrm{H}), 7.26-6.90(\mathrm{~m}, 10 \mathrm{H}), 4.30(\mathrm{~d}, J=10.7 \mathrm{~Hz}, 1 \mathrm{H}), 4.09(\mathrm{dd}, J=10.7,1.3$ $\mathrm{Hz}, 2 \mathrm{H}), 3.83-3.76(\mathrm{~m}, 1 \mathrm{H}), 3.66(\mathrm{dd}, J=9.8,2.8 \mathrm{~Hz}, 1 \mathrm{H}), 3.15(\mathrm{t}, J=9.4 \mathrm{~Hz}, 1 \mathrm{H})$, 3.11-3.04 (m, 1H), $2.39(\mathrm{dd}, J=13.5,7.9 \mathrm{~Hz}, 1 \mathrm{H}) ;{ }^{13} \mathrm{C} \mathrm{NMR}\left(100 \mathrm{MHz}, \mathrm{CDCl}_{3}\right)$ $\delta / \mathrm{ppm}=148.8,143.5,142.4,142.3,127.8,127.7,127.0,125.9,125.7,125.4,125.3$, 
123.2, 59.3, 59.2, 51.7, 43.4, 10.5Spectral data are in good agreement with literature values. $^{8}$

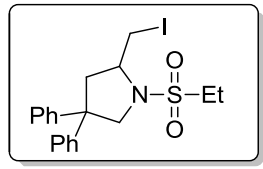

1-(Ethylsulfonyl)-2-(iodomethyl)-4,4-diphenylpyrrolidine (4h). Compound $\mathbf{4 h}$ was prepared according to the general procedure and isolated as a yellow solid (177 $\mathrm{mg}$, $78 \%$ yield) after flash chromatography (petroleum ether/ethyl acetate $=13 / 1) . \mathrm{mp}=$ 118-120 ${ }^{\circ} \mathrm{C} .{ }^{1} \mathrm{H}$ NMR $\left(400 \mathrm{MHz}, \mathrm{CDCl}_{3}\right) \delta / \mathrm{ppm}=7.41(\mathrm{~d}, J=7.6 \mathrm{~Hz}, 2 \mathrm{H}), 7.32(\mathrm{dd}, J$ $=15.7,7.6 \mathrm{~Hz}, 4 \mathrm{H}), 7.24-7.19(\mathrm{~m}, 4 \mathrm{H}), 4.45(\mathrm{~d}, J=10.8 \mathrm{~Hz}, 1 \mathrm{H}), 4.03(\mathrm{~d}, J=10.8$ $\mathrm{Hz}, 1 \mathrm{H}), 3.84(\mathrm{q}, J=8.6 \mathrm{~Hz}, 1 \mathrm{H}), 3.57(\mathrm{dd}, J=10.1,2.6 \mathrm{~Hz}, 1 \mathrm{H}), 3.31(\mathrm{dd}, J=10.0$, $7.9 \mathrm{~Hz}, 1 \mathrm{H}), 3.12$ (dd, $J=12.9,6.6 \mathrm{~Hz}, 1 \mathrm{H}), 2.76(\mathrm{dt}, J=14.7,7.3 \mathrm{~Hz}, 1 \mathrm{H}), 2.71-$ $2.62(\mathrm{~m}, 1 \mathrm{H}), 2.58(\mathrm{dd}, J=13.0,8.5 \mathrm{~Hz}, 1 \mathrm{H}), 1.26(\mathrm{t}, J=7.4 \mathrm{~Hz}, 3 \mathrm{H}) .{ }^{13} \mathrm{C}$ NMR $(100$ $\left.\mathrm{MHz}, \mathrm{CDCl}_{3}\right) \delta / \mathrm{ppm}=143.7,143.1,127.8,127.7,126.0,125.8,125.7,125.5,58.4$, 58.2, 52.2, 45.8, 43.8, 11.7, 7.0. ESI-HRMS: calc. for $\left[\mathrm{C}_{19} \mathrm{H}_{22} \mathrm{INO}_{2} \mathrm{~S}+\mathrm{H}\right]^{+}: \mathrm{m} / \mathrm{z}=$ 456.0494, found: 456.0488 .

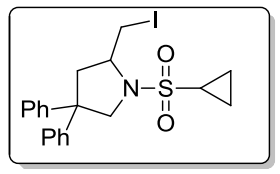

1-(Cyclopropylsulfonyl)-2-(iodomethyl)-4,4-diphenylpyrrolidine (4i). Compound 4i was prepared according to the general procedure and isolated as a white solid (215 mg, $92 \%$ yield) after a wash with saturated aqueous $\mathrm{Na}_{2} \mathrm{~S}_{2} \mathrm{O}_{3}$ and evaporation.mp = 125-127 ${ }^{\circ} \mathrm{C} .{ }^{1} \mathrm{H}$ NMR $\left(400 \mathrm{MHz}, \mathrm{CDCl}_{3}\right) \delta=7.32(\mathrm{~d}, J=7.5 \mathrm{~Hz}, 2 \mathrm{H}), 7.26-7.18(\mathrm{~m}$, $4 \mathrm{H}), 7.18-7.10(\mathrm{~m}, 4 \mathrm{H}), 4.28(\mathrm{dd}, J=10.8,1.4 \mathrm{~Hz}, 1 \mathrm{H}), 4.11(\mathrm{~d}, J=10.8 \mathrm{~Hz}, 1 \mathrm{H})$, $3.94-3.85(\mathrm{~m}, 1 \mathrm{H}), 3.52(\mathrm{dd}, J=9.8,2.8 \mathrm{~Hz}, 1 \mathrm{H}), 3.19-3.09(\mathrm{~m}, 1 \mathrm{H}), 2.46(\mathrm{dd}, J=$ $13.2,8.0 \mathrm{~Hz}, 1 \mathrm{H}), 1.75(\mathrm{tt}, J=8.0,4.9 \mathrm{~Hz}, 1 \mathrm{H}), 1.28-1.16(\mathrm{~m}, 1 \mathrm{H}), 1.04-0.91(\mathrm{~m}$, $2 \mathrm{H}), 0.61(\mathrm{dd}, J=8.0,1.5 \mathrm{~Hz}, 2 \mathrm{H}) .{ }^{13} \mathrm{C} \mathrm{NMR}\left(100 \mathrm{MHz}, \mathrm{CDCl}_{3}\right) \delta / \mathrm{ppm}=143.8$, 143.4, 127.8, 127.7, 125.9, 125.8, 125.8, 125.5, 58.7, 58.5, 52.2, 43.6, 27.5, 11.4, 4.2, 3.7. ESI-HRMS: calc. for $\left[\mathrm{C}_{20} \mathrm{H}_{22} \mathrm{INO}_{2} \mathrm{~S}+\mathrm{H}\right]^{+}: \mathrm{m} / \mathrm{z}=468.0494$, found: 468.0469 .

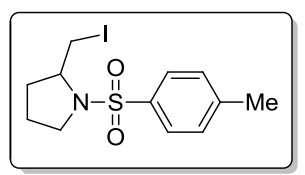

2-(Iodomethyl)-1-tosylpyrrolidine $(\mathbf{4} \mathbf{j})$. Compound $\mathbf{4 j}$ was prepared according to the general procedure and isolated as a white solid (162 $\mathrm{mg}, 89 \%$ yield) after a wash with saturated aqueous $\mathrm{Na}_{2} \mathrm{~S}_{2} \mathrm{O}_{3}$ and evaporation. $\mathrm{mp}=90^{\circ} \mathrm{C} ;{ }^{1} \mathrm{H} \mathrm{NMR}\left(400 \mathrm{MHz}, \mathrm{CDCl}_{3}\right.$ ) $\delta / \mathrm{ppm}=7.65(\mathrm{~d}, J=8.0 \mathrm{~Hz}, 2 \mathrm{H}), 7.26(\mathrm{~d}, J=8.0 \mathrm{~Hz}, 2 \mathrm{H}), 3.65(\mathrm{dd}, J=11.2,5.4 \mathrm{~Hz}$, $1 \mathrm{H}), 3.53(\mathrm{dd}, J=9.5,2.5 \mathrm{~Hz}, 1 \mathrm{H}), 3.40(\mathrm{dd}, J=10.0,5.9 \mathrm{~Hz}, 1 \mathrm{H}), 3.21-3.06(\mathrm{~m}, 2 \mathrm{H})$, $2.36(\mathrm{~s}, 3 \mathrm{H}), 1.88-1.64(\mathrm{~m}, 3 \mathrm{H}), 1.44(\mathrm{dd}, J=11.3,5.9 \mathrm{~Hz}, 1 \mathrm{H}) ;{ }^{13} \mathrm{C} \mathrm{NMR}(100 \mathrm{MHz}$, $\left.\mathrm{CDCl}_{3}\right) \delta / \mathrm{ppm}=143.8,134.1,129.9,127.5,60.7,50.1,31.9,23.9,21.6,11.7$. Spectral data are in good agreement with literature values. 


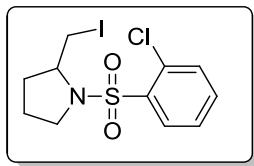

1-((2-Chlorophenyl)sulfonyl)-2-(iodomethyl)pyrrolidine (4k). Compound 4k was prepared according to the general procedure and isolated as a white solid (165 $\mathrm{mg}, 86 \%$ yield) after flash chromatography (petroleum ether/ethyl acetate $=20 / 1$ ). $\mathrm{mp}=$ 48-50 ${ }^{\circ} \mathrm{C} .{ }^{1} \mathrm{H}$ NMR $\left(400 \mathrm{MHz}, \mathrm{CDCl}_{3}\right) \delta / \mathrm{ppm}=8.08(\mathrm{dd}, J=7.9,1.4 \mathrm{~Hz}, 1 \mathrm{H}), 7.51$ $(\mathrm{dd}, J=12.2,4.8 \mathrm{~Hz}, 2 \mathrm{H}), 7.43-7.38(\mathrm{~m}, 1 \mathrm{H}), 4.19-4.11(\mathrm{~m}, 1 \mathrm{H}), 3.49$ (ddd, $J=$ 12.4, 7.5, $4.8 \mathrm{~Hz}, 2 \mathrm{H}), 3.44-3.37(\mathrm{~m}, 1 \mathrm{H}), 3.18(\mathrm{t}, J=9.7 \mathrm{~Hz}, 1 \mathrm{H}), 2.08(\mathrm{dt}, J=17.4$, $7.4 \mathrm{~Hz}, 1 \mathrm{H}), 2.01-1.91(\mathrm{~m}, 2 \mathrm{H}), 1.85-1.75(\mathrm{~m}, 1 \mathrm{H}) .{ }^{13} \mathrm{C} \mathrm{NMR}\left(100 \mathrm{MHz}, \mathrm{CDCl}_{3}\right)$ $\delta / \mathrm{ppm}=135.8,132.8,131.2,131.11,131.10,126.1,59.7,48.6,31.2,23.1,9.7$. ESI-HRMS: calc. for $\left[\mathrm{C}_{11} \mathrm{H}_{13} \mathrm{CIINO}_{2} \mathrm{~S}+\mathrm{H}\right]^{+}: \mathrm{m} / \mathrm{z}=385.9478$, found: 385.9491 .

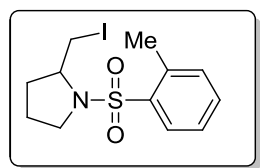

2-(Iodomethyl)-1-(o-tolylsulfonyl)pyrrolidine (41). Compound 41 was prepared according to the general procedure and isolated as a white solid (151 $\mathrm{mg}, 83 \%$ yield) after flash chromatography (petroleum ether/ethyl acetate $=20 / 1$ ). $\mathrm{mp}=36-37{ }^{\circ} \mathrm{C} ;{ }^{1} \mathrm{H}$ NMR $\left(400 \mathrm{MHz}, \mathrm{CDCl}_{3}\right) \delta / \mathrm{ppm}=7.85(\mathrm{~d}, J=8.3 \mathrm{~Hz}, 1 \mathrm{H}), 7.40(\mathrm{t}, J=7.5 \mathrm{~Hz}, 1 \mathrm{H})$, 7.27-7.23 (m, 2H), 4.01-3.90 (m, 1H), $3.37(\mathrm{dd}, J=9.8,2.9 \mathrm{~Hz}, 1 \mathrm{H}), 3.32-3.20(\mathrm{~m}$, $2 \mathrm{H}), 3.07(\mathrm{t}, J=9.5 \mathrm{~Hz}, 1 \mathrm{H}), 2.60(\mathrm{~s}, 3 \mathrm{H}), 2.06-1.95(\mathrm{~m}, 1 \mathrm{H}), 1.94-1.80(\mathrm{~m}, 2 \mathrm{H})$, $1.80-1.66(\mathrm{~m}, 1 \mathrm{H}) ;{ }^{13} \mathrm{C} \mathrm{NMR}\left(100 \mathrm{MHz}, \mathrm{CDCl}_{3}\right) \delta / \mathrm{ppm}=138.1,136.9,133.1,132.9$, 129.6, 126.3, 59.8, 49.9, 32.4, 24.2, 20.9, 11.3 Spectral data are in good agreement with literature values. ${ }^{8}$

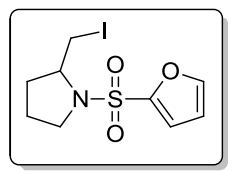

1-(Furan-2-ylsulfonyl)-2-(iodomethyl)pyrrolidine $\quad \mathbf{4 m}$ ). Compound $\mathbf{4 m}$ was prepared according to the general procedure and isolated as a white solid (147 $\mathrm{mg}, 86 \%$ yield) after flash chromatography (petroleum ether/ethyl acetate $=10 / 1$ ). $\mathrm{mp}=$ 100-102 ${ }^{\circ} \mathrm{C} .{ }^{1} \mathrm{H}$ NMR $\left(400 \mathrm{MHz}, \mathrm{CDCl}_{3}\right) \delta / \mathrm{ppm}=7.61(\mathrm{dd}, J=7.0,4.4 \mathrm{~Hz}, 2 \mathrm{H}), 7.14$ $(\mathrm{dd}, J=4.9,3.8 \mathrm{~Hz}, 1 \mathrm{H}), 3.77-3.69(\mathrm{~m}, 1 \mathrm{H}), 3.58(\mathrm{dd}, J=9.7,3.1 \mathrm{~Hz}, 1 \mathrm{H}), 3.52(\mathrm{dd}$, $J=10.5,5.9 \mathrm{~Hz}, 1 \mathrm{H}), 3.24(\mathrm{t}, J=9.7 \mathrm{~Hz}, 2 \mathrm{H}), 1.87(\mathrm{dd}, J=17.2,10.8 \mathrm{~Hz}, 3 \mathrm{H}), 1.58$ $-1.49(\mathrm{~m}, 1 \mathrm{H}) .{ }^{13} \mathrm{C} \mathrm{NMR}\left(100 \mathrm{MHz}, \mathrm{CDCl}_{3}\right) \delta / \mathrm{ppm}=135.8,131.5,131.1,126.6,60.1$, 49.3, 31.0, 22.9, 10.3. ESI-HRMS: calc. for $\left[\mathrm{C}_{9} \mathrm{H}_{12} \mathrm{INO}_{3} \mathrm{~S}_{2}+\mathrm{H}\right]^{+}: \mathrm{m} / \mathrm{z}=341.9661$, found: 341.9653 .

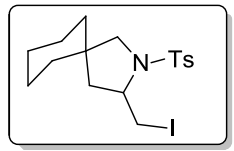

3-(Iodomethyl)-2-tosyl-2-azaspiro[4.5]decane (4n). Compound 4n was prepared 
according to the general procedure and isolated as a white solid (197 $\mathrm{mg}, 91 \%$ yield) after flash chromatography (petroleum ether/ethyl acetate $=25 / 1$ ) $. \mathrm{m} .=114-116^{\circ} \mathrm{C}$; ${ }^{1} \mathrm{H}$ NMR $\left(400 \mathrm{MHz}, \mathrm{CDCl}_{3}\right) \delta / \mathrm{ppm}=7.67(\mathrm{~d}, J=8.0 \mathrm{~Hz}, 2 \mathrm{H}), 7.26(\mathrm{~d}, J=8.0 \mathrm{~Hz}$, $2 \mathrm{H}), 3.69(\mathrm{dd}, J=9.5,2.7 \mathrm{~Hz}, 1 \mathrm{H}), 3.54(\mathrm{td}, J=8.8,2.6 \mathrm{~Hz}, 1 \mathrm{H}), 3.37-3.23(\mathrm{~m}, 2 \mathrm{H})$, $3.09(\mathrm{~d}, J=11.0 \mathrm{~Hz}, 1 \mathrm{H}), 2.36(\mathrm{~s}, 3 \mathrm{H}), 1.92(\mathrm{dd}, J=12.9,7.2 \mathrm{~Hz}, 1 \mathrm{H}), 1.45(\mathrm{dd}, J=$ 13.0, $8.6 \mathrm{~Hz}, 1 \mathrm{H}), 1.39-1.26(\mathrm{~m}, 4 \mathrm{H}), 1.20-0.95(\mathrm{~m}, 4 \mathrm{H}), 0.72-0.65(\mathrm{~m}, 1 \mathrm{H}), 0.54(\mathrm{dd}$, $J=11.5,5.9 \mathrm{~Hz}, 1 \mathrm{H}) ;{ }^{13} \mathrm{C} \mathrm{NMR}\left(100 \mathrm{MHz}, \mathrm{CDCl}_{3}\right) \delta / \mathrm{ppm}=143.7,134.7,129.7$, 127.5, 59.3, 59.2, 45.9, 41.4, 36.1, 33.9, 25.9, 23.7, 22.7, 21.6, 13.7; Spectral data are in good agreement with literature values. ${ }^{8}$

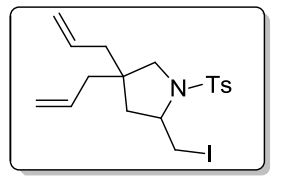

4,4-Diallyl-2-(iodomethyl)-1-tosylpyrrolidine (4o). Compound 4o was prepared according to the general procedure and isolated as an oil (178 $\mathrm{mg}, 80 \%$ yield) after flash chromatography (petroleum ether/ethyl acetate $=30 / 1) .{ }^{1} \mathrm{H}$ NMR $(400 \mathrm{MHz}$, $\left.\mathrm{CDCl}_{3}\right) \delta / \mathrm{ppm}=7.67(\mathrm{~d}, J=8.2 \mathrm{~Hz}, 2 \mathrm{H}), 7.26(\mathrm{~d}, J=8.0 \mathrm{~Hz}, 2 \mathrm{H}), 5.67-5.56(\mathrm{~m}, 1 \mathrm{H})$, 5.49-5.39 (m, 1H), 5.05-4.88 (m, 3H), $4.73(\mathrm{~d}, J=16.9 \mathrm{~Hz}, 1 \mathrm{H}), 3.68-3.56(\mathrm{~m}, 2 \mathrm{H})$, $3.33(\mathrm{t}, J=8.8 \mathrm{~Hz}, 1 \mathrm{H}), 3.22-3.11(\mathrm{~m}, 2 \mathrm{H}), 2.36(\mathrm{~s}, 3 \mathrm{H}), 2.03(\mathrm{~d}, J=7.3 \mathrm{~Hz}, 2 \mathrm{H})$, $1.93(\mathrm{dd}, J=13.1,7.2 \mathrm{~Hz}, 1 \mathrm{H}), 1.62-1.50(\mathrm{~m}, 2 \mathrm{H}), 1.42(\mathrm{dd}, J=14.0,7.9 \mathrm{~Hz}, 1 \mathrm{H})$; ${ }^{13} \mathrm{C} \mathrm{NMR}\left(100 \mathrm{MHz}, \mathrm{CDCl}_{3}\right) \delta / \mathrm{ppm}=143.9,135.1,133.5,133.1,129.8,127.5,118.7$, $59.4,58.7,43.7,43.3,40.4,39.2,21.6,13.5$. Spectral data are in good agreement with literature values. ${ }^{8}$

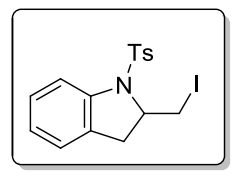

2-(Iodomethyl)-1-tosylindoline (4p). Compound $4 p$ was prepared according to the general procedure and isolated as a white solid (159 $\mathrm{mg}, 77 \%$ yield) after flash chromatography (petroleum ether/ethyl acetate $=25 / 1$ ). $\mathrm{mp}=151-153{ }^{\circ} \mathrm{C} ;{ }^{1} \mathrm{H}$ NMR $\left(400 \mathrm{MHz}, \mathrm{CDCl}_{3}\right) \delta / \mathrm{ppm}=7.57(\mathrm{~d}, J=8.1 \mathrm{~Hz}, 1 \mathrm{H}), 7.48(\mathrm{~d}, J=8.1 \mathrm{~Hz}, 2 \mathrm{H})$, 7.20-7.07 (m, 3H), 7.00-6.93 (m, 2H), 4.30-4.24 (m, 1H),3.58 (dd, $J=9.7,3.4 \mathrm{~Hz}$, $1 \mathrm{H}), 3.18(\mathrm{t}, J=9.9 \mathrm{~Hz}, 1 \mathrm{H}), 2.86(\mathrm{dd}, \mathrm{J}=16.7,9.3 \mathrm{~Hz}, 1 \mathrm{H}), 2.76(\mathrm{dd}, J=16.7,3.0$ $\mathrm{Hz}, 1 \mathrm{H}), 2.27(\mathrm{~s}, 3 \mathrm{H}) ;{ }^{13} \mathrm{C} \mathrm{NMR}\left(100 \mathrm{MHz}, \mathrm{CDCl}_{3}\right) \delta / \mathrm{ppm}=144.3,141.2,134.4$, $130.5,129.8,128.1,127.1,125.3,124.9,116.8,62.5,34.9,21.6,11.6$; Spectral data are in good agreement with literature values. ${ }^{8}$

$* * * * * * * * * * * * * * * * * * * * * * * * * * * * * * * * * * * * * * * * * * * * * * * * * * * * * * * * * * * * * * * * * * * * * * * *$

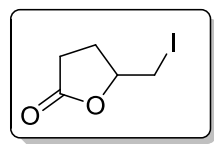

5-(Iodomethyl)dihydrofuran-2(3H)-one (6a). Compound $\mathbf{6 a}$ was prepared according to the general procedure and isolated as a yellow oil (107 $\mathrm{mg}, 95 \%$ yield) after a wash with saturated aqueous $\mathrm{Na}_{2} \mathrm{~S}_{2} \mathrm{O}_{3}$ and evaporation. ${ }^{1} \mathrm{H}$ NMR (400 MHz, $\left.\mathrm{CDCl}_{3}\right) \delta / \mathrm{ppm}=4.56-4.48(\mathrm{~m}, 1 \mathrm{H}), 3.36(\mathrm{dd}, J=10.5,4.6 \mathrm{~Hz}, 1 \mathrm{H}), 3.29(\mathrm{dd}, J=$ 
10.5, $6.6 \mathrm{~Hz}, 1 \mathrm{H}), 2.69-2.49(\mathrm{~m}, 2 \mathrm{H}), 2.49-2.38(\mathrm{~m}, 1 \mathrm{H}), 2.03-1.90(\mathrm{~m}, 1 \mathrm{H}) .{ }^{13} \mathrm{C}$ NMR $\left(100 \mathrm{MHz}, \mathrm{CDCl}_{3}\right) \delta / \mathrm{ppm}=176.4,78.5,28.9,28.1$, 8.0. Spectral data are in good agreement with literature values. ${ }^{9}$

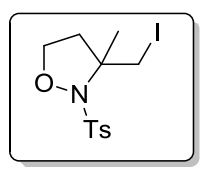

3-(Iodomethyl)-3-methyl-2-tosylisoxazolidine (6b). Compound $6 \mathbf{b}$ was prepared according to the general procedure and isolated as a white solid (171 mg, 90\% yield) after flash chromatography (petroleum ether/ethyl acetate $=15 / 1$ ). $\mathrm{mp}=79-81{ }^{\circ} \mathrm{C} .{ }^{1} \mathrm{H}$ NMR (400 MHz, $\left.\mathrm{CDCl}_{3}\right) \delta / \mathrm{ppm}=7.78(\mathrm{~d}, J=8.3 \mathrm{~Hz}, 2 \mathrm{H}), 7.25(\mathrm{~d}, J=8.3 \mathrm{~Hz}, 2 \mathrm{H})$, $4.22(\mathrm{dd}, J=15.1,7.4 \mathrm{~Hz}, 1 \mathrm{H}), 3.92(\mathrm{dd}, J=14.5,7.5 \mathrm{~Hz}, 1 \mathrm{H}), 3.45(\mathrm{~d}, J=10.2 \mathrm{~Hz}$, $1 \mathrm{H}), 3.37(\mathrm{~d}, J=10.2 \mathrm{~Hz}, 1 \mathrm{H}), 2.49(\mathrm{ddd}, J=12.4,8.5,6.2 \mathrm{~Hz}, 1 \mathrm{H}), 2.37-2.31(\mathrm{~m}$, $4 \mathrm{H}), 1.81(\mathrm{~s}, 3 \mathrm{H}) .{ }^{13} \mathrm{C} \mathrm{NMR}\left(100 \mathrm{MHz}, \mathrm{CDCl}_{3}\right) \delta / \mathrm{ppm}=144.8,135.2,129.5,128.9$, 69.3, 69.2, 41.2, 23.2, 21.7, 16.3. ESI-HRMS: calc. for $\left[\mathrm{C}_{12} \mathrm{H}_{16} \mathrm{INO}_{3} \mathrm{~S}+\mathrm{H}\right]^{+}: \mathrm{m} / \mathrm{z}=$ 381.9974, found: 381.9964 .

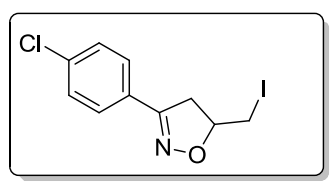

3-(4-Chlorophenyl)-5-(iodomethyl)-5-methyl-4,5-dihydroisoxazole

$(6 \mathbf{c})$.

Compound $\mathbf{6 c}$ was prepared according to the general procedure and isolated as a white solid (133 mg, 83\% yield) after flash chromatography (petroleum ether/ethyl acetate $=18 / 1) . \mathrm{mp}=82-84{ }^{\circ} \mathrm{C} .{ }^{1} \mathrm{H}$ NMR $\left(400 \mathrm{MHz}, \mathrm{CDCl}_{3}\right) \delta / \mathrm{ppm}=7.53(\mathrm{~d}, J=8.6$ $\mathrm{Hz}, 2 \mathrm{H}), 7.31(\mathrm{~d}, J=8.6 \mathrm{~Hz}, 2 \mathrm{H}), 4.94-4.79(\mathrm{~m}, 1 \mathrm{H}), 3.42(\mathrm{dd}, J=17.0,10.4 \mathrm{~Hz}$, $1 \mathrm{H}), 3.35(\mathrm{dd}, J=10.1,4.1 \mathrm{~Hz}, 1 \mathrm{H}), 3.19-3.15(\mathrm{~m}, 1 \mathrm{H}), 3.12(\mathrm{dd}, J=15.2,4.8 \mathrm{~Hz}$, 1H). ${ }^{13} \mathrm{C}$ NMR $\left(100 \mathrm{MHz}, \mathrm{CDCl}_{3}\right) \delta / \mathrm{ppm}=153.9,135.3,128.1,127.0,126.6,79.6$, $39.9,6.4$. Spectral data are in good agreement with literature values. ${ }^{10}$

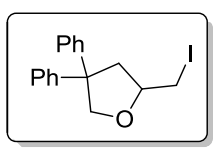

2-(Iodomethyl)-4,4-diphenyltetrahydrofuran (6d). Compound 6d was prepared according to the general procedure and isolated as an oil (142 mg, 78\% yield) after flash chromatography (petroleum ether/ethyl acetate $=50 / 1$ ). $\mathrm{mp}=54-55{ }^{\circ} \mathrm{C} ;{ }^{1} \mathrm{H}$ NMR $\left(400 \mathrm{MHz}, \mathrm{CDCl}_{3}\right) \delta=7.29-6.94(\mathrm{~m}, 10 \mathrm{H}), 4.61(\mathrm{dd}, J=8.8,0.8 \mathrm{~Hz}, 1 \mathrm{H}), 4.12$ $(\mathrm{d}, J=8.8 \mathrm{~Hz}, 1 \mathrm{H}), 4.06-3.94(\mathrm{~m}, 1 \mathrm{H}), 3.19(\mathrm{dd}, J=9.9,5.0 \mathrm{~Hz}, 1 \mathrm{H}), 3.13(\mathrm{dd}, J=$ 9.9, $6.8 \mathrm{~Hz}, 1 \mathrm{H}), 2.66(\mathrm{dd}, J=12.2,5.9 \mathrm{~Hz}, 1 \mathrm{H}), 2.34(\mathrm{dd}, J=12.3,9.1 \mathrm{~Hz}, 1 \mathrm{H}) ;{ }^{13} \mathrm{C}$ NMR $\left(100 \mathrm{MHz}, \mathrm{CDCl}_{3}\right) \delta=145.8,145.0,128.6,128.5,127.2,127.0,126.8,126.6$, 77.9, 77.5, 56.5, 45.3, 10.7. Spectral data are in good agreement with literature values. $^{8}$

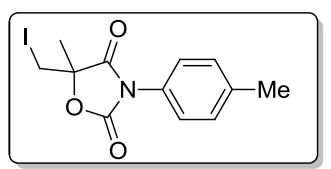


5-(Iodomethyl)-5-methyl-3-(p-tolyl)oxazolidine-2,4-dione (6e). Compound 6e was prepared according to the general procedure and isolated as a white solid (140 mg, 81\% yield) after flash chromatography (petroleum ether/ethyl acetate $=15 / 1) ; \mathrm{mp}=106$ $108{ }^{\circ} \mathrm{C} .{ }^{1} \mathrm{H}$ NMR $\left(400 \mathrm{MHz}, \mathrm{CDCl}_{3}\right) \delta / \mathrm{ppm}=7.32(\mathrm{~d}, J=8.6 \mathrm{~Hz}, 2 \mathrm{H}), 7.29(\mathrm{~d}, J=8.6$ $\mathrm{Hz}, 2 \mathrm{H}), 3.67(\mathrm{~d}, J=11.3 \mathrm{~Hz}, 1 \mathrm{H}), 3.51(\mathrm{~d}, J=11.3 \mathrm{~Hz}, 1 \mathrm{H}), 2.39$ (s, 3H), 1.83 (s, $3 \mathrm{H}) .{ }^{13} \mathrm{C}$ NMR $\left(100 \mathrm{MHz}, \mathrm{CDCl}_{3}\right) \delta / \mathrm{ppm}=171.5,151.9,138.4,129.0,127.0,124.6$, $82.1,20.7,20.2,5.4$. Spectral data are in good agreement with literature values. ${ }^{3}$

******************************************************************************<smiles>OC(CI)c1ccccc1</smiles>

2-Iodo-1-phenylethanol (8a). Compound 8a was prepared according to the general procedure and isolated as an oil (103 $\mathrm{mg}, 83 \%$ yield) after flash chromatography (petroleum ether/ethyl acetate $=8 / 1) .{ }^{1} \mathrm{H} \mathrm{NMR}\left(400 \mathrm{MHz}, \mathrm{CDCl}_{3}\right) \delta / \mathrm{ppm}=7.39-7.32$ $(\mathrm{m}, 5 \mathrm{H}), 4.82(\mathrm{dd}, J=5.4,3.2 \mathrm{~Hz}, 1 \mathrm{H}), 3.48(\mathrm{dd}, J=10.3,3.7 \mathrm{~Hz}, 1 \mathrm{H}), 3.39$ (dd, $J=$ 10.3, 8.7 Hz, 1H), $2.73(\mathrm{~d}, J=2.7 \mathrm{~Hz}, 1 \mathrm{H}) .{ }^{13} \mathrm{C} \mathrm{NMR}\left(100 \mathrm{MHz}, \mathrm{CDCl}_{3}\right) \delta / \mathrm{ppm}=$ $140.1,127.6,127.3,124.7,73.0,14.2$. Spectral data are in good agreement with literature values. $^{11}$

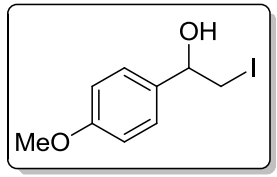

2-Iodo-1-(4-methoxyphenyl)ethanol (8b). Compound $8 \mathrm{~b}$ was prepared according to the general procedure and isolated as an oil (118 $\mathrm{mg}, 85 \%$ yield) after flash chromatography (petroleum ether/ethyl acetate $=5 / 1) .{ }^{1} \mathrm{H}$ NMR $\left(400 \mathrm{MHz}, \mathrm{CDCl}_{3}\right)$ $\delta / \mathrm{ppm}=7.18(\mathrm{~d}, J=8.7 \mathrm{~Hz}, 2 \mathrm{H}), 6.79(\mathrm{~d}, J=8.7 \mathrm{~Hz}, 2 \mathrm{H}), 4.72-4.63(\mathrm{~m}, 1 \mathrm{H}), 3.71(\mathrm{~s}$, $3 \mathrm{H}), 3.36-3.27(\mathrm{~m}, 2 \mathrm{H}), 2.62(\mathrm{~d}, J=2.6 \mathrm{~Hz}, 1 \mathrm{H}) .{ }^{13} \mathrm{C} \mathrm{NMR}\left(100 \mathrm{MHz}, \mathrm{CDCl}_{3}\right)$ $\delta / \mathrm{ppm}=159.56,133.4,127.1,114.1,73.7,55.4,15.5$. Spectral data are in good agreement with literature values. ${ }^{12}$

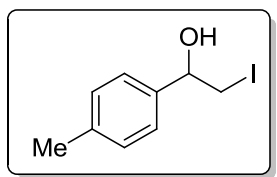

2-Iodo-1-(p-tolyl)ethanol (8c). Compound 8c was prepared according to the general procedure and isolated as an oil (120 mg, 92\% yield) after flash chromatography (petroleum ether/ethyl acetate $=10 / 1) .{ }^{1} \mathrm{H} \mathrm{NMR}\left(400 \mathrm{MHz}, \mathrm{CDCl}_{3}\right) \delta / \mathrm{ppm}=7.15(\mathrm{~d}, J$ $=8.1 \mathrm{~Hz}, 2 \mathrm{H}), 7.08(\mathrm{~d}, J=8.1 \mathrm{~Hz}, 2 \mathrm{H}), 4.72-4.63(\mathrm{~m}, 1 \mathrm{H}), 3.34(\mathrm{dd}, J=10.3,3.9$ $\mathrm{Hz}, 1 \mathrm{H}), 3.27(\mathrm{dd}, J=10.2,8.6 \mathrm{~Hz}, 1 \mathrm{H}), 2.65-2.56(\mathrm{~m}, 1 \mathrm{H}), 2.26(\mathrm{~s}, 3 \mathrm{H}) .{ }^{13} \mathrm{C} \mathrm{NMR}$ $\left(100 \mathrm{MHz}, \mathrm{CDCl}_{3}\right) \delta / \mathrm{ppm}=137.2,137.1,128.3,124.7,72.8,20.2$, 14.3. Spectral data are in good agreement with literature values. ${ }^{12}$ 


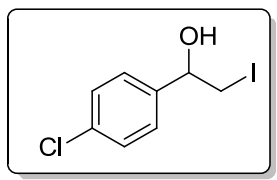

1-(4-Chlorophenyl)-2-iodoethanol (8d). Compound 8d was prepared according to the general procedure and isolated as white solid (127 $\mathrm{mg}, 90 \%$ yield) after a wash with saturated aqueous $\mathrm{Na}_{2} \mathrm{~S}_{2} \mathrm{O}_{3}$ and evaporation. $\mathrm{mp}=78-80{ }^{\circ} \mathrm{C} .{ }^{1} \mathrm{H} \mathrm{NMR}(400 \mathrm{MHz}$, $\left.\mathrm{CDCl}_{3}\right) \delta / \mathrm{ppm}=7.33(\mathrm{~d}, J=8.5 \mathrm{~Hz}, 2 \mathrm{H}), 7.29(\mathrm{~d}, J=8.5 \mathrm{~Hz}, 2 \mathrm{H}), 4.78(\mathrm{dd}, J=8.5$, $3.3 \mathrm{~Hz}, 1 \mathrm{H}), 3.44(\mathrm{dd}, J=10.3,3.8 \mathrm{~Hz}, 1 \mathrm{H}), 3.34(\mathrm{dd}, J=10.3,8.5 \mathrm{~Hz}, 1 \mathrm{H}), 2.73(\mathrm{~s}$, $1 \mathrm{H}) .{ }^{13} \mathrm{C}$ NMR $\left(100 \mathrm{MHz}, \mathrm{CDCl}_{3}\right) \delta / \mathrm{ppm}=138.6,133.0,127.8,126.1,72.2,13.9$. Spectral data are in good agreement with literature values. ${ }^{12}$

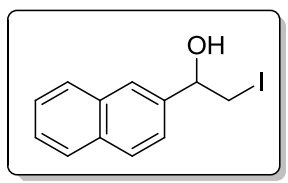

2-Iodo-1-(naphthalen-2-yl)ethanol (8e). Compound 8e was prepared according to the general procedure and isolated as a white solid (130 mg, 87\% yield) after a wash with saturated aqueous $\mathrm{Na}_{2} \mathrm{~S}_{2} \mathrm{O}_{3}$ and evaporation. $\mathrm{mp}=46-48{ }^{\circ} \mathrm{C} .{ }^{1} \mathrm{H} \mathrm{NMR}(400 \mathrm{MHz}$, $\left.\mathrm{CDCl}_{3}\right) \delta / \mathrm{ppm}=7.70(\mathrm{dd}, \mathrm{J}=9.2,3.5 \mathrm{~Hz}, 4 \mathrm{H}), 7.38(\mathrm{dd}, \mathrm{J}=6.3,3.2 \mathrm{~Hz}, 2 \mathrm{H}), 7.30(\mathrm{dd}$, $\mathrm{J}=8.5,1.6 \mathrm{~Hz}, 1 \mathrm{H}), 4.82(\mathrm{dd}, \mathrm{J}=8.4,3.3 \mathrm{~Hz}, 1 \mathrm{H}), 3.41(\mathrm{dd}, \mathrm{J}=10.3,3.7 \mathrm{~Hz}, 1 \mathrm{H})$, $3.33(\mathrm{dd}, \mathrm{J}=10.3,8.6 \mathrm{~Hz}, 1 \mathrm{H}), 2.73(\mathrm{~s}, 1 \mathrm{H}) .{ }^{13} \mathrm{C} \mathrm{NMR}\left(100 \mathrm{MHz}, \mathrm{CDCl}_{3}\right) \delta / \mathrm{ppm}=$ 137.4, 132.1, 132.1, 127.5, 127.0, 126.7, 125.4, 125.2, 123.9, 122.4, 73.0, 14.0. Spectral data are in good agreement with literature values. ${ }^{13}$

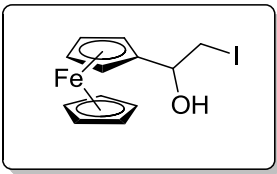

Cyclopenta-2,4-dien-1-yl(2-(1-hydroxy-2-iodoethyl)cyclopenta-2,4-dien-1-yl)iron (8f). Compound $\mathbf{8 f}$ was prepared according to the general procedure and isolated as an oil (160 mg, 90\% yield) after a wash with saturated aqueous $\mathrm{Na}_{2} \mathrm{~S}_{2} \mathrm{O}_{3}$ and evaporation. ${ }^{1} \mathrm{H}$ NMR $\left(400 \mathrm{MHz}, \mathrm{CDCl}_{3}\right) \delta / \mathrm{ppm}=4.50-4.20(\mathrm{~m}, 10 \mathrm{H}), 3.43(\mathrm{dd}, J=9.9,3.1 \mathrm{~Hz}$, $1 \mathrm{H}), 3.30(\mathrm{dd}, J=18.8,9.2 \mathrm{~Hz}, 1 \mathrm{H}), 2.34(\mathrm{~s}, 1 \mathrm{H}) .{ }^{13} \mathrm{C} \mathrm{NMR}\left(100 \mathrm{MHz}, \mathrm{CDCl}_{3}\right)$ $\delta / \mathrm{ppm}=68.9, \quad 68.8, \quad 68.2,67.8, \quad 66.9,64.8, \quad 11.7$. ESI-HRMS: calc. for $\left[\mathrm{C}_{12} \mathrm{H}_{13} \mathrm{FeIO}+\mathrm{H}\right]^{+}: \mathrm{m} / \mathrm{z}=355.9361$, found: 355.9343 .

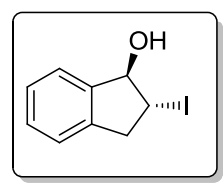

anti-2-Iodo-2,3-dihydro-1H-inden-1-ol (8g). Compound 8g was prepared according to the general procedure and isolated as a colorless solid (98 $\mathrm{mg}, 75 \%$ yield) after flash chromatography (petroleum ether/ethyl acetate $=12 / 1) . \mathrm{mp}=114-116{ }^{\circ} \mathrm{C} .{ }^{1} \mathrm{H}$ NMR (400 MHz, $\left.\mathrm{CDCl}_{3}\right) \delta / \mathrm{ppm}=7.43-7.40(\mathrm{~m}, 1 \mathrm{H}), 7.27(\mathrm{dd}, J=8.7,5.2 \mathrm{~Hz}, 2 \mathrm{H})$, $7.24-7.20(\mathrm{~m}, 1 \mathrm{H}), 5.38(\mathrm{t}, J=5.6 \mathrm{~Hz}, 1 \mathrm{H}), 4.19(\mathrm{dd}, J=14.3,7.4 \mathrm{~Hz}, 1 \mathrm{H}), 3.58(\mathrm{dd}$, $J=16.2,7.3 \mathrm{~Hz}, 1 \mathrm{H}), 3.30(\mathrm{dd}, J=16.2,8.0 \mathrm{~Hz}, 1 \mathrm{H}), 2.53(\mathrm{~d}, J=5.6 \mathrm{~Hz}, 1 \mathrm{H}) .{ }^{13} \mathrm{C}$ 
NMR $\left(100 \mathrm{MHz}, \mathrm{CDCl}_{3}\right) \delta / \mathrm{ppm}=142.1,141.0,128.8,127.6,124.4,123.9,85.0,42.3$, 30.2. Spectral data are in good agreement with literature values. ${ }^{12}$

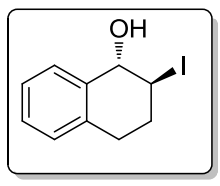

anti-2-Iodo-1,2,3,4-tetrahydronaphthalen-1-ol $(\mathbf{8 h})$. Compound $8 \mathrm{~h}$ was prepared according to the general procedure and isolated as an oil (114 mg, 83\% yield) after flash chromatography (petroleum ether/ethyl acetate $=8 / 1) .{ }^{1} \mathrm{H}$ NMR $(400 \mathrm{MHz}$, $\left.\mathrm{CDCl}_{3}\right) \delta / \mathrm{ppm}=7.41-7.37(\mathrm{~m}, 1 \mathrm{H}), 7.18-7.14(\mathrm{~m}, 2 \mathrm{H}), 7.05-7.01(\mathrm{~m}, 1 \mathrm{H}), 4.94-$ $4.86(\mathrm{~m}, 1 \mathrm{H}), 4.44-4.35(\mathrm{~m}, 1 \mathrm{H}), 2.83(\mathrm{t}, J=6.3 \mathrm{~Hz}, 2 \mathrm{H}), 2.46(\mathrm{~s}, 1 \mathrm{H}), 2.41-2.33$ $(\mathrm{m}, 1 \mathrm{H}), 2.22(\mathrm{dt}, J=13.9,6.7 \mathrm{~Hz}, 1 \mathrm{H}) .{ }^{13} \mathrm{C} \mathrm{NMR}\left(100 \mathrm{MHz}, \mathrm{CDCl}_{3}\right) \delta / \mathrm{ppm}=134.2$, 134.1, 127.7, 127.6, 127.1, 125.6, 74.0, 35.0, 29.7, 28.2. Spectral data are in good agreement with literature values. ${ }^{14}$

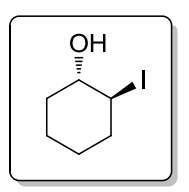

anti-2-Iodocyclohexanol (8i). Compound $\mathbf{8 i}$ was prepared according to the general procedure and isolated as an oil (104 $\mathrm{mg}, 92 \%$ yield) after a wash with saturated aqueous $\mathrm{Na}_{2} \mathrm{~S}_{2} \mathrm{O}_{3}$ and evaporation. ${ }^{1} \mathrm{H}$ NMR $\left(400 \mathrm{MHz}, \mathrm{CDCl}_{3}\right) \delta / \mathrm{ppm}=3.98(\mathrm{ddd}, J$ $=12.3,9.7,4.3 \mathrm{~Hz}, 1 \mathrm{H}), 3.60(\mathrm{td}, J=9.8,4.2 \mathrm{~Hz}, 1 \mathrm{H}), 2.62(\mathrm{~s}, 1 \mathrm{H}), 2.45-2.37(\mathrm{~m}$, $1 \mathrm{H}), 2.08-2.02(\mathrm{~m}, 1 \mathrm{H}), 2.01-1.91(\mathrm{~m}, 1 \mathrm{H}), 1.81-1.74(\mathrm{~m}, 1 \mathrm{H}), 1.50-1.41(\mathrm{~m}$, $1 \mathrm{H}), 1.37-1.16(\mathrm{~m}, 3 \mathrm{H}) .{ }^{13} \mathrm{C} \mathrm{NMR}\left(100 \mathrm{MHz}, \mathrm{CDCl}_{3}\right) \delta / \mathrm{ppm}=74.8,42.1,37.5,32.8$, $26.9,23.4$. Spectral data are in good agreement with literature values. ${ }^{12}$

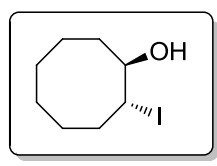

anti-2-Iodocyclooctanol (8j). Compound $\mathbf{8 j}$ was prepared according to the general procedure and isolated as an oil (93 $\mathrm{mg}, 73 \%$ yield) after flash chromatography (petroleum ether/ethyl acetate $=20 / 1) .{ }^{1} \mathrm{H} \mathrm{NMR}\left(400 \mathrm{MHz}, \mathrm{CDCl}_{3}\right) \delta / \mathrm{ppm}=4.46(\mathrm{ddd}$, $J=9.7,6.9,3.0 \mathrm{~Hz}, 1 \mathrm{H}), 4.07(\mathrm{dd}, J=9.4,7.6 \mathrm{~Hz}, 1 \mathrm{H}), 2.29-2.18(\mathrm{~m}, 2 \mathrm{H}), 2.17-$ $2.06(\mathrm{~m}, 1 \mathrm{H}), 2.04-1.94(\mathrm{~m}, 1 \mathrm{H}), 1.70(\mathrm{ddd}, J=10.0,7.7,3.8 \mathrm{~Hz}, 5 \mathrm{H}), 1.55(\mathrm{ddd}, J$ $=24.7,10.9,3.8 \mathrm{~Hz}, 2 \mathrm{H}), 1.45-1.33(\mathrm{~m}, 2 \mathrm{H}) .{ }^{13} \mathrm{C} \mathrm{NMR}\left(100 \mathrm{MHz}, \mathrm{CDCl}_{3}\right) \delta / \mathrm{ppm}=$ $77.2,49.4,33.2,31.3,25.8,24.8,24.6,24.4$. Spectral data are in good agreement with literature values. ${ }^{15}$

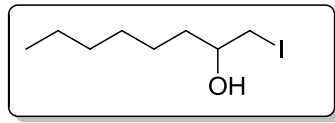

1-Iodooctan-2-ol (8k). Compound $\mathbf{8 k}$ was prepared according to the general procedure and isolated as an oil $(83 \mathrm{mg}, 65 \%$ yield) after flash chromatography (petroleum ether/ethyl acetate $=15 / 1) .{ }^{1} \mathrm{H}$ NMR $\left(400 \mathrm{MHz}, \mathrm{CDCl}_{3}\right) \delta / \mathrm{ppm}=3.45(\mathrm{~d}, J$ 
$=4.3 \mathrm{~Hz}, 1 \mathrm{H}), 3.32(\mathrm{dd}, J=10.2,3.5 \mathrm{~Hz}, 1 \mathrm{H}), 3.17(\mathrm{dd}, J=10.1,6.7 \mathrm{~Hz}, 1 \mathrm{H}), 2.12(\mathrm{~d}$, $J=4.5 \mathrm{~Hz}, 1 \mathrm{H}), 1.48(\mathrm{dd}, J=13.5,6.3 \mathrm{~Hz}, 2 \mathrm{H}), 1.33-1.14(\mathrm{~m}, 8 \mathrm{H}), 0.82(\mathrm{t}, J=6.7$ $\mathrm{Hz}, 3 \mathrm{H}) .{ }^{13} \mathrm{C} \mathrm{NMR}\left(100 \mathrm{MHz}, \mathrm{CDCl}_{3}\right) \delta / \mathrm{ppm}=70.0,35.6,30.7,28.1,24.6,21.6,15.8$, 13.1. Spectral data are in good agreement with literature values. ${ }^{14}$

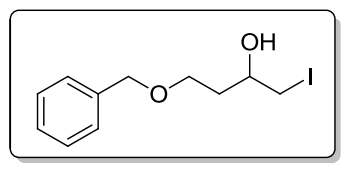

4-(Benzyloxy)-1-iodobutan-2-ol (81). Compound 81 was prepared according to the general procedure and isolated as an oil (67 $\mathrm{mg}, 44 \%$ yield) after flash chromatography (petroleum ether/ethyl acetate $=10 / 1) .{ }^{1} \mathrm{H}$ NMR $\left(400 \mathrm{MHz}, \mathrm{CDCl}_{3}\right)$ $\delta / \mathrm{ppm}=7.32-7.18(\mathrm{~m}, 5 \mathrm{H}), 4.43(\mathrm{~s}, 2 \mathrm{H}), 3.75-3.67(\mathrm{~m}, 1 \mathrm{H}), 3.62(\mathrm{ddd}, J=9.7,6.4$, $4.7 \mathrm{~Hz}, 1 \mathrm{H}), 3.56(\mathrm{ddd}, J=9.5,7.2,4.6 \mathrm{~Hz}, 1 \mathrm{H}), 3.22(\mathrm{dd}, J=10.1,4.9 \mathrm{~Hz}, 1 \mathrm{H}), 3.15$ $(\mathrm{dd}, J=10.1,6.1 \mathrm{~Hz}, 2 \mathrm{H}), 1.88-1.72(\mathrm{~m}, 2 \mathrm{H}) \cdot{ }^{13} \mathrm{C} \mathrm{NMR}\left(100 \mathrm{MHz}, \mathrm{CDCl}_{3}\right)$ $\delta / \mathrm{ppm}=136.7,127.5,126.8,126.7,72.3,69.1,66.9,34.8,13.3$. ESI-HRMS: calc. for $\left[\mathrm{C}_{11} \mathrm{H}_{15} \mathrm{IO}_{2}+\mathrm{H}\right]^{+}: \mathrm{m} / \mathrm{z}=307.0195$, found: 307.0163 .

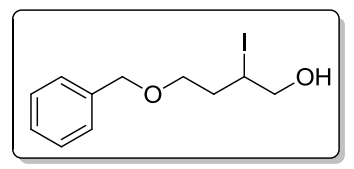

4-(Benzyloxy)-2-iodobutan-1-ol (81'). Compound 81' was prepared according to the general procedure and isolated as an oil (40 $\mathrm{mg}, 26 \%$ yield) after flash chromatography (petroleum ether/ethyl acetate $=10 / 1) .{ }^{1} \mathrm{H}$ NMR $\left(400 \mathrm{MHz}, \mathrm{CDCl}_{3}\right)$ $\delta / \mathrm{ppm}=7.33-7.19(\mathrm{~m}, 5 \mathrm{H}), 4.45(\mathrm{~s}, 2 \mathrm{H}), 4.38-4.30(\mathrm{~m}, 1 \mathrm{H}), 3.69(\mathrm{t}, J=6.2 \mathrm{~Hz}$, $2 \mathrm{H}), 3.63-3.56(\mathrm{~m}, 1 \mathrm{H}), 3.54-3.46(\mathrm{~m}, 1 \mathrm{H}), 2.76(\mathrm{t}, J=6.9 \mathrm{~Hz}, 1 \mathrm{H}), 2.16-2.00(\mathrm{~m}$, 2H). ${ }^{13} \mathrm{C} \mathrm{NMR}\left(100 \mathrm{MHz}, \mathrm{CDCl}_{3}\right) \delta / \mathrm{ppm}=136.7,127.5,126.9,126.8,72.3,68.2,67.4$, 35.6, 35.4. ESI-HRMS: calc. for $\left[\mathrm{C}_{11} \mathrm{H}_{15} \mathrm{IO}_{2}+\mathrm{H}\right]^{+}: \mathrm{m} / \mathrm{z}=307.0195$, found: 307.0175 .

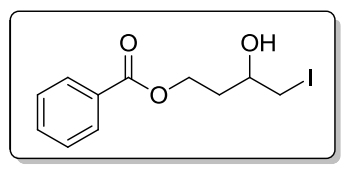

3-Hydroxy-4-iodobutyl benzoate $(\mathbf{8 m})$. Compound $\mathbf{8 m w a s}$ prepared according to the general procedure and isolated as an oil $(80 \mathrm{mg}$, 50\% yield) after flash chromatography (petroleum ether/ethyl acetate $=5 / 1) .{ }^{1} \mathrm{H} \mathrm{NMR}\left(400 \mathrm{MHz}, \mathrm{CDCl}_{3}\right)$ $\delta / \mathrm{ppm}=7.99-7.91(\mathrm{~m}, 2 \mathrm{H}), 7.54-7.45(\mathrm{~m}, 1 \mathrm{H}), 7.41-7.33(\mathrm{~m}, 2 \mathrm{H}), 4.52-4.43(\mathrm{~m}$, $1 \mathrm{H}), 4.37(\mathrm{dt}, J=11.1,5.4 \mathrm{~Hz}, 1 \mathrm{H}), 3.74-3.64(\mathrm{~m}, 1 \mathrm{H}), 3.34(\mathrm{dd}, J=10.3,4.0 \mathrm{~Hz}$, $1 \mathrm{H}), 3.22(\mathrm{dd}, J=10.3,6.5 \mathrm{~Hz}, 1 \mathrm{H}), 2.65-2.57(\mathrm{~m}, 1 \mathrm{H}), 2.09-1.97(\mathrm{~m}, 1 \mathrm{H}), 1.91-$ $1.81(\mathrm{~m}, 1 \mathrm{H}) .{ }^{13} \mathrm{C} \mathrm{NMR}\left(100 \mathrm{MHz}, \mathrm{CDCl}_{3}\right) \delta / \mathrm{ppm}=165.8,132.1,128.9,128.6,127.4$, 66.9, 60.6, 34.8, 14.2. ESI-HRMS: calc. for $\left[\mathrm{C}_{11} \mathrm{H}_{13} \mathrm{IO}_{3}+\mathrm{H}\right]^{+}: \mathrm{m} / \mathrm{z}=320.9988$, found: 320.9947.

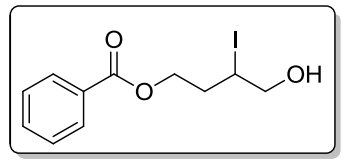


4-Hydroxy-3-iodobutyl benzoate (8m'). Compound 8m' was prepared according to the general procedure and isolated as an oil (45 $\mathrm{mg}, 28 \%$ yield) after flash chromatography (petroleum ether/ethyl acetate $=5 / 1) .{ }^{1} \mathrm{H} \mathrm{NMR}\left(400 \mathrm{MHz}, \mathrm{CDCl}_{3}\right)$ $\delta / \mathrm{ppm}=8.04-7.97(\mathrm{~m}, 2 \mathrm{H}), 7.55-7.48(\mathrm{~m}, 1 \mathrm{H}), 7.43-7.35(\mathrm{~m}, 2 \mathrm{H}), 5.14-5.07(\mathrm{~m}$, $1 \mathrm{H}), 3.67(\mathrm{dt}, J=10.2,5.1 \mathrm{~Hz}, 1 \mathrm{H}), 3.63-3.54(\mathrm{~m}, 1 \mathrm{H}), 3.46(\mathrm{dd}, J=10.8,4.5 \mathrm{~Hz}$, $1 \mathrm{H}), 3.38(\mathrm{dd}, J=10.8,5.6 \mathrm{~Hz}, 1 \mathrm{H}), 2.36(\mathrm{~s}, 1 \mathrm{H}), 2.04-1.86(\mathrm{~m}, 2 \mathrm{H}) .{ }^{13} \mathrm{C}$ NMR $(100$ $\left.\mathrm{MHz}, \mathrm{CDCl}_{3}\right) \delta / \mathrm{ppm}=165.5,132.5,128.9,128.4,127.5,69.3,57.3,36.6,7.5$. ESI-HRMS: calc. for $\left[\mathrm{C}_{11} \mathrm{H}_{13} \mathrm{IO}_{3}+\mathrm{H}\right]^{+}: \mathrm{m} / \mathrm{z}=320.9988$, found: 320.9959 .

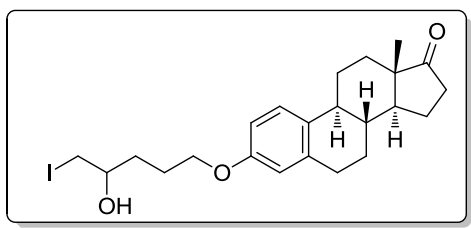

$(8 R, 9 S, 13 S, 14 S)-3-((4-H y d r o x y-5-i o d o p e n t y l) o x y)-13-m e t h y l-7,8,9,11,12,13,15,16-$ octahydro-6H-cyclopenta[a]phenanthren-17(14H)-one (8n).Compound $8 \mathrm{n}$ was prepared according to the general procedure and isolated as an oil (118 $\mathrm{mg}, 49 \%$ yield) after flash chromatography (petroleum ether/ethyl acetate $=4 / 1) .{ }^{1} \mathrm{H}$ NMR $(400 \mathrm{MHz}$, $\left.\mathrm{CDCl}_{3}\right) \delta / \mathrm{ppm}=7.12(\mathrm{~d}, J=8.6 \mathrm{~Hz}, 1 \mathrm{H}), 6.66-6.60(\mathrm{~m}, 1 \mathrm{H}), 6.59-6.54(\mathrm{~m}, 1 \mathrm{H})$, $3.91(\mathrm{t}, J=5.8 \mathrm{~Hz}, 2 \mathrm{H}), 3.60-3.51(\mathrm{~m}, 1 \mathrm{H}), 3.33(\mathrm{dd}, J=10.2,3.8 \mathrm{~Hz}, 1 \mathrm{H}), 3.19(\mathrm{dd}$, $J=10.2,6.7 \mathrm{~Hz}, 1 \mathrm{H}), 2.87-2.75(\mathrm{~m}, 2 \mathrm{H}), 2.43(\mathrm{dd}, J=18.8,8.5 \mathrm{~Hz}, 1 \mathrm{H}), 2.33-$ $2.29(\mathrm{~m}, 1 \mathrm{H}), 2.13-1.96(\mathrm{~m}, 2 \mathrm{H}), 1.94-1.69(\mathrm{~m}, 5 \mathrm{H}), 1.68-1.48(\mathrm{~m}, 3 \mathrm{H}), 1.44-$ $1.32(\mathrm{~m}, 4 \mathrm{H}), 1.28-1.15(\mathrm{~m}, 2 \mathrm{H}), 0.83(\mathrm{~s}, 3 \mathrm{H}) .{ }^{13} \mathrm{C} \mathrm{NMR}\left(100 \mathrm{MHz}, \mathrm{CDCl}_{3}\right) \delta / \mathrm{ppm}=$ 220, 155.7, 136.8, 131.2, 125.3, 113.5, 111.0, 69.6, 66.5, 49.3, 47.0, 42.9, 37.3, 34.9, $32.4,30.5,28.6,25.9,25.5,24.9,24.6,20.6,15.1,12.8$. ESI-HRMS: calc. for $\left[\mathrm{C}_{23} \mathrm{H}_{31} \mathrm{IO}_{3}+\mathrm{H}\right]^{+}: \mathrm{m} / \mathrm{z}=483.1396$, found: 483.1370 .

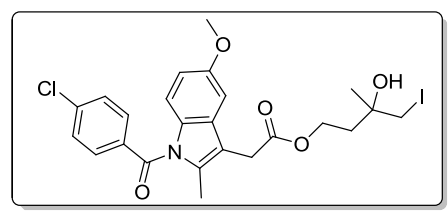

\section{3-Hydroxy-4-iodo-3-methylbutyl}

\section{2-(1-(4-chlorobenzoyl)-5-methoxy-2-methyl-1H-indol-3-yl)acetate}

(8o).

Compound 80 was prepared according to the general procedure and isolated as an oil (160 mg, 56\% yield) after flash chromatography (petroleum ether/ethyl acetate $=3 / 1$ ). ${ }^{1} \mathrm{H}$ NMR $\left(400 \mathrm{MHz}, \mathrm{CDCl}_{3}\right) \delta / \mathrm{ppm}=7.67(\mathrm{~d}, J=8.6 \mathrm{~Hz}, 2 \mathrm{H}), 7.47(\mathrm{~d}, J=10.8 \mathrm{~Hz}$, $2 \mathrm{H}), 6.93(\mathrm{~d}, J=2.5 \mathrm{~Hz}, 1 \mathrm{H}), 6.84(\mathrm{~d}, J=9.0 \mathrm{~Hz}, 1 \mathrm{H}), 6.66(\mathrm{dd}, J=9.0,2.5 \mathrm{~Hz}, 1 \mathrm{H})$, $4.30-4.23(\mathrm{~m}, 2 \mathrm{H}), 3.83(\mathrm{~s}, 3 \mathrm{H}), 3.67(\mathrm{~s}, 2 \mathrm{H}), 3.25(\mathrm{~d}, J=10.3 \mathrm{~Hz}, 1 \mathrm{H}), 3.20(\mathrm{~d}, J=$ $10.3 \mathrm{~Hz}, 1 \mathrm{H}), 2.39$ (s, 3H), $2.04(\mathrm{~s}, 1 \mathrm{H}), 2.01-1.97(\mathrm{~m}, 2 \mathrm{H}), 1.30(\mathrm{~s}, 3 \mathrm{H}) .{ }^{13} \mathrm{C}$ NMR $\left(100 \mathrm{MHz}, \mathrm{CDCl}_{3}\right) \delta / \mathrm{ppm}=169.6,167.3,155.0,138.3,134.9,132.8,130.2,129.8$, 129.5, 128.1, 114.0, 111.3, 110.7, 100.1, 68.8, 60.6, 54.7, 37.4, 29.4, 25.4, 20.8, 12.3. ESI-HRMS: calc. for $\left[\mathrm{C}_{24} \mathrm{H}_{25} \mathrm{ClINO}_{5}+\mathrm{H}\right]^{+}: \mathrm{m} / \mathrm{z}=570.0544$, found: 570.0536 .

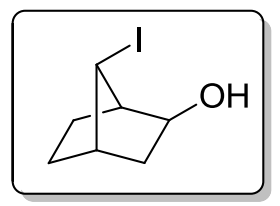


7-Iodobicyclo[2.2.1]heptan-2-ol (8p). Compound 8p was prepared according to the general procedure and isolated as an oil (79 $\mathrm{mg}, 66 \%$ yield) after flash chromatography (petroleum ether/ethyl acetate $=10 / 1) .{ }^{1} \mathrm{H}$ NMR $\left(400 \mathrm{MHz}, \mathrm{CDCl}_{3}\right)$ $\delta / \mathrm{ppm}={ }^{1} \mathrm{H}$ NMR $\left(400 \mathrm{MHz}, \mathrm{CDCl}_{3}\right) \delta 3.82-3.74(\mathrm{~m}, 1 \mathrm{H}), 3.66(\mathrm{~s}, 1 \mathrm{H}), 2.45(\mathrm{~d}, J=$ $3.4 \mathrm{~Hz}, 1 \mathrm{H}), 2.39(\mathrm{~s}, 1 \mathrm{H}), 2.24(\mathrm{~d}, J=10.5 \mathrm{~Hz}, 1 \mathrm{H}), 2.02-1.94(\mathrm{~m}, 2 \mathrm{H}), 1.57-1.48$ $(\mathrm{m}, 2 \mathrm{H}), 1.20-1.10(\mathrm{~m}, 1 \mathrm{H}), 1.00-0.92(\mathrm{~m}, 1 \mathrm{H}) .{ }^{13} \mathrm{C} \mathrm{NMR}\left(100 \mathrm{MHz}, \mathrm{CDCl}_{3}\right)$ $\delta / \mathrm{ppm}=75.6,48.9,43.0,41.6,29.3,24.7,23.9$. Spectral data are in good agreement with literature values. ${ }^{12}$

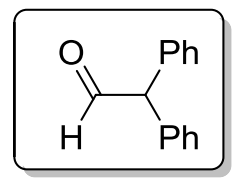

2,2-Diphenylacetaldehyde. (8q). Compound 8q was prepared according to the general procedure and isolated as an oil (82 $\mathrm{mg}, 63 \%$ yield) after flash chromatography (petroleum ether/ethyl acetate $=18 / 1) .{ }^{1} \mathrm{H}$ NMR $\left(400 \mathrm{MHz}, \mathrm{CDCl}_{3}\right)$ $\delta / \mathrm{ppm}=9.95(\mathrm{~d}, J=2.5 \mathrm{~Hz}, 1 \mathrm{H}), 7.40-7.35(\mathrm{~m}, 4 \mathrm{H}), 7.34-7.29(\mathrm{~m}, 2 \mathrm{H}), 7.25-7.19$ $(\mathrm{m}, 4 \mathrm{H}), 4.89$ (d, $J=2.5 \mathrm{~Hz}, 1 \mathrm{H}) .{ }^{13} \mathrm{C} \mathrm{NMR}\left(100 \mathrm{MHz}, \mathrm{CDCl}_{3}\right) \delta / \mathrm{ppm}=198.6,136.3$, 129.2, 129.0, 127.7, 64.1.Spectral data are in good agreement with literature values. ${ }^{12}$

*************************************************************************<smiles>CCOc1ccccc1C[IH]CCCO</smiles>

(1-Ethoxy-2-iodoethyl)benzene (9a). Compound 9a was prepared according to the general procedure and isolated as an oil (113 $\mathrm{mg}, 82 \%$ yield) after flash chromatography (petroleum ether/ethyl acetate $=30 / 1) .{ }^{1} \mathrm{H}$ NMR $\left(400 \mathrm{MHz}, \mathrm{CDCl}_{3}\right)$ $\delta / \mathrm{ppm}=7.44-7.29(\mathrm{~m}, 5 \mathrm{H}), 4.42(\mathrm{dd}, J=8.0,5.0 \mathrm{~Hz}, 1 \mathrm{H}), 3.51-3.41(\mathrm{~m}, 2 \mathrm{H}), 3.38$ - $3.32(\mathrm{~m}, 2 \mathrm{H}), 1.24(\mathrm{t}, J=7.0 \mathrm{~Hz}, 3 \mathrm{H}) .{ }^{13} \mathrm{C} \mathrm{NMR}\left(100 \mathrm{MHz}, \mathrm{CDCl}_{3}\right) \delta / \mathrm{ppm}=139.5$, $127.6,127.2,125.4,80.8,64.0,14.2,9.9$. Spectral data are in good agreement with literature values. ${ }^{16}$

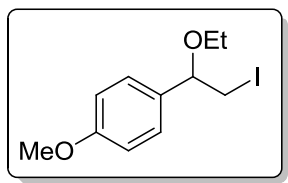

1-(1-Ethoxy-2-iodoethyl)-4-methoxybenzene (9b). Compound 9b was prepared according to the general procedure and isolated as an oil (113 $\mathrm{mg}, 74 \%$ yield) after flash chromatography (petroleum ether/ethyl acetate $=20 / 1) .{ }^{1} \mathrm{H}$ NMR $(400 \mathrm{MHz}$, $\left.\mathrm{CDCl}_{3}\right) \delta / \mathrm{ppm}=7.15(\mathrm{~d}, J=8.6 \mathrm{~Hz}, 1 \mathrm{H}), 6.80(\mathrm{~d}, J=8.6 \mathrm{~Hz}, 1 \mathrm{H}), 4.28(\mathrm{dd}, J=8.3$, $4.8 \mathrm{~Hz}, 1 \mathrm{H}), 3.72(\mathrm{~s}, 3 \mathrm{H}), 3.32(\mathrm{dd}, J=18.1,10.5 \mathrm{~Hz}, 2 \mathrm{H}), 3.26-3.17(\mathrm{~m}, 2 \mathrm{H}), 1.12$ $(\mathrm{t}, J=7.0 \mathrm{~Hz}, 3 \mathrm{H}) .{ }^{13} \mathrm{C} \mathrm{NMR}\left(100 \mathrm{MHz}, \mathrm{CDCl}_{3}\right) \delta / \mathrm{ppm}=158.5,131.5,126.6,112.9$, $80.3,63.7,54.2,14.2,10.3$. Spectral data are in good agreement with literature values. $^{17}$ 


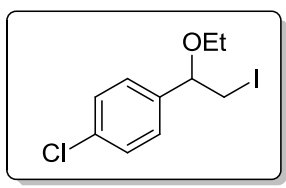

1-Chloro-4-(2-ethoxy-1-iodoethyl)benzene (9c). Compound 9c was prepared according to the general procedure and isolated as an oil (119 mg, 77\% yield) after flash chromatography (petroleum ether/ethyl acetate $=60 / 1) .{ }^{1} \mathrm{H}$ NMR $(400 \mathrm{MHz}$, $\left.\mathrm{CDCl}_{3}\right) \delta / \mathrm{ppm}=7.26(\mathrm{~d}, J=8.5 \mathrm{~Hz}, 2 \mathrm{H}), 7.19(\mathrm{~d}, J=8.5 \mathrm{~Hz}, 2 \mathrm{H}), 4.29(\mathrm{dd}, J=7.7$, $5.1 \mathrm{~Hz}, 1 \mathrm{H}), 3.35$ (q, $J=7.0 \mathrm{~Hz}, 2 \mathrm{H}), 3.27-3.17(\mathrm{~m}, 2 \mathrm{H}), 1.14(\mathrm{t}, J=7.0 \mathrm{~Hz}, 3 \mathrm{H})$. ${ }^{13} \mathrm{C} \mathrm{NMR}\left(100 \mathrm{MHz}, \mathrm{CDCl}_{3}\right) \delta / \mathrm{ppm}=138.0,133.0,127.8,126.9,80.0,64.1,14.1,9.4$. Spectral data are in good agreement with literature values. Spectral data are in good agreement with literature values. ${ }^{17}$

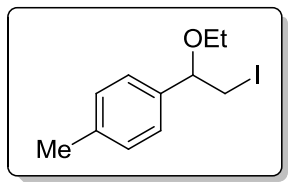

1-(1-Ethoxy-2-iodoethyl)-4-methylbenzene (9d). Compound 9d was prepared according to the general procedure and isolated as an oil (129 $\mathrm{mg}, 89 \%$ yield) after a wash with saturated aqueous $\mathrm{Na}_{2} \mathrm{~S}_{2} \mathrm{O}_{3}$ and evaporation. ${ }^{1} \mathrm{H} \mathrm{NMR}\left(400 \mathrm{MHz}, \mathrm{CDCl}_{3}\right.$ ) $\delta / \mathrm{ppm}=7.12(\mathrm{~d}, J=8.1 \mathrm{~Hz}, 1 \mathrm{H}), 7.08(\mathrm{~d}, J=8.0 \mathrm{~Hz}, 1 \mathrm{H}), 4.29(\mathrm{dd}, J=8.2,4.8 \mathrm{~Hz}$, $1 \mathrm{H}), 3.34$ (tdd, $J=9.3,7.0,2.2 \mathrm{~Hz}, 2 \mathrm{H}), 3.23(\mathrm{dd}, J=8.2,6.6 \mathrm{~Hz}, 2 \mathrm{H}), 2.25(\mathrm{~s}, 3 \mathrm{H})$, $1.13(\mathrm{t}, J=7.0 \mathrm{~Hz}, 3 \mathrm{H}) .{ }^{13} \mathrm{C}$ NMR $\left(100 \mathrm{MHz}, \mathrm{CDCl}_{3}\right) \delta / \mathrm{ppm}=137.0,136.5,128.3$, 125.4, 80.6, 63.8, 20.2, 14.1, 10.1. ESI-HRMS: calc. for $\left[\mathrm{C}_{11} \mathrm{H}_{15} \mathrm{IO}+\mathrm{H}\right]^{+}: \mathrm{m} / \mathrm{z}=$ 291.0246, found: 291.0220 .

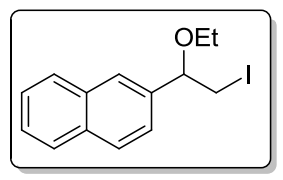

2-(1-Ethoxy-2-iodoethyl)naphthalene (9e). Compound 9e was prepared according to the general procedure and isolated as an oil (114 $\mathrm{mg}, 70 \%$ yield) after flash chromatography (petroleum ether/ethyl acetate $=70 / 1) .{ }^{1} \mathrm{H}$ NMR $\left(400 \mathrm{MHz}, \mathrm{CDCl}_{3}\right)$ $\delta / \mathrm{ppm}=7.75(\mathrm{~d}, J=8.1 \mathrm{~Hz}, 3 \mathrm{H}), 7.68(\mathrm{~s}, 1 \mathrm{H}), 7.41-7.37(\mathrm{~m}, 2 \mathrm{H}), 7.34(\mathrm{dd}, J=8.5$, $1.5 \mathrm{~Hz}, 1 \mathrm{H}), 4.47(\mathrm{dd}, J=8.3,4.8 \mathrm{~Hz}, 1 \mathrm{H}), 3.40-3.36(\mathrm{~m}, 2 \mathrm{H}), 3.35-3.26(\mathrm{~m}, 2 \mathrm{H})$, $1.15(\mathrm{t}, J=7.0 \mathrm{~Hz}, 3 \mathrm{H}) .{ }^{13} \mathrm{C} \mathrm{NMR}\left(100 \mathrm{MHz}, \mathrm{CDCl}_{3}\right) \delta / \mathrm{ppm}=136.8,132.3,132.1$, 127.6, 126.9, 126.7, 125.3, 125.1, 124.9, 122.8, 80.9, 64.1, 14.2, 9.7. ESI-HRMS: calc. for $\left[\mathrm{C}_{14} \mathrm{H}_{15} \mathrm{IO}+\mathrm{H}\right]^{+}: \mathrm{m} / \mathrm{z}=327.0246$, found: 327.02565 .

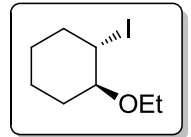

anti-1-Ethoxy-2-iodocyclohexane (9f). Compound 9f was prepared according to the general procedure and isolated as an oil (110 $\mathrm{mg}, 87 \%$ yield) after a wash with saturated aqueous $\mathrm{Na}_{2} \mathrm{~S}_{2} \mathrm{O}_{3}$ and evaporation. ${ }^{1} \mathrm{H} \mathrm{NMR}\left(400 \mathrm{MHz}, \mathrm{CDCl}_{3}\right) \delta / \mathrm{ppm}=$ $4.04-3.98(\mathrm{~m}, 1 \mathrm{H}), 3.61(\mathrm{dq}, J=14.0,7.0 \mathrm{~Hz}, 1 \mathrm{H}), 3.49(\mathrm{dq}, J=14.0,7.0 \mathrm{~Hz}, 1 \mathrm{H})$, 
$3.27(\mathrm{td}, J=8.9,4.0 \mathrm{~Hz}, 1 \mathrm{H}), 2.44-2.30(\mathrm{~m}, 1 \mathrm{H}), 2.14-2.04(\mathrm{~m}, 1 \mathrm{H}), 1.94(\mathrm{dt}, J=$ 21.1, 7.3 Hz, 1H), $1.83-1.72(\mathrm{~m}, 1 \mathrm{H}), 1.55-1.45(\mathrm{~m}, 1 \mathrm{H}), 1.34-1.23(\mathrm{~m}, 3 \mathrm{H}), 1.19$ $(\mathrm{t}, J=7.0 \mathrm{~Hz}, 3 \mathrm{H}) .{ }^{13} \mathrm{C} \mathrm{NMR}\left(100 \mathrm{MHz}, \mathrm{CDCl}_{3}\right) \delta / \mathrm{ppm}=81.4,63.8,37.0,35.1,30.4$, $26.2,22.7,14.6$. Spectral data are in good agreement with literature values. ${ }^{18}$

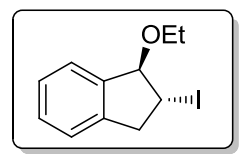

anti-1-Ethoxy-2-iodo-2,3-dihydro-1H-indene $(9 \mathrm{~g})$. Compound $9 \mathrm{~g}$ was prepared according to the general procedure and isolated as an oil (190 mg, 66\% yield) after flash chromatography (petroleum ether/ethyl acetate $=60 / 1) .{ }^{1} \mathrm{H}$ NMR $(400 \mathrm{MHz}$, $\left.\mathrm{CDCl}_{3}\right) \delta / \mathrm{ppm}=7.32(\mathrm{~d}, J=6.5 \mathrm{~Hz}, 1 \mathrm{H}), 7.19(\mathrm{dt}, J=7.6,6.0 \mathrm{~Hz}, 2 \mathrm{H}), 7.13(\mathrm{~d}, J=$ $6.8 \mathrm{~Hz}, 1 \mathrm{H}), 5.08(\mathrm{~d}, J=3.9 \mathrm{~Hz}, 1 \mathrm{H}), 4.40-4.31(\mathrm{~m}, 1 \mathrm{H}), 3.78(\mathrm{dd}, J=9.2,7.0 \mathrm{~Hz}$, $1 \mathrm{H}), 3.68-3.59(\mathrm{~m}, 2 \mathrm{H}), 3.19(\mathrm{dd}, J=17.0,5.0 \mathrm{~Hz}, 1 \mathrm{H}), 1.18(\mathrm{t}, J=7.0 \mathrm{~Hz}, 3 \mathrm{H}) .{ }^{13} \mathrm{C}$ NMR $\left(100 \mathrm{MHz}, \mathrm{CDCl}_{3}\right) \delta / \mathrm{ppm}=140.2$, 139.6, 127.9, 126.1, 123.9, 123.6, 90.8, 64.7, $42.4,25.5,14.7$. Spectral data are in good agreement with literature values. ${ }^{18}$

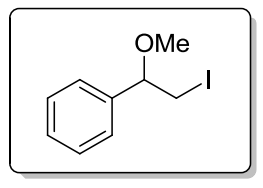

(2-Iodo-1-methoxyethyl)benzene (9h). Compound $9 \mathrm{~h}$ was prepared according to the general procedure and isolated as an oil (110 $\mathrm{mg}$, 84\% yield) after flash chromatography (petroleum ether/ethyl acetate $=10 / 1) .{ }^{1} \mathrm{H} \mathrm{NMR}\left(400 \mathrm{MHz}, \mathrm{CDCl}_{3}\right)$ $\delta / \mathrm{ppm}=7.30-7.28(\mathrm{~m}, 1 \mathrm{H}), 7.28-7.24(\mathrm{~m}, 2 \mathrm{H}), 7.24-7.21(\mathrm{~m}, 2 \mathrm{H}), 4.21(\mathrm{dd}, J=$ 7.8, $4.9 \mathrm{~Hz}, 1 \mathrm{H}), 3.24-3.22(\mathrm{~m}, 1 \mathrm{H}), 3.22-3.21(\mathrm{~m}, 1 \mathrm{H}), 3.21(\mathrm{~s}, 3 \mathrm{H}) .{ }^{13} \mathrm{C} \mathrm{NMR}$ $\left(100 \mathrm{MHz}, \mathrm{CDCl}_{3}\right) \delta / \mathrm{ppm}=138.7,127.6,127.4,125.5,82.5,56.3$, 9.5. Spectral data are in good agreement with literature values. ${ }^{16}$

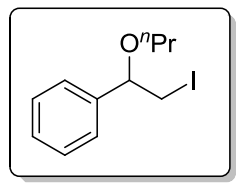

(1-Iodo-2-propoxyethyl)benzene (9i). Compound 9i was prepared according to the general procedure and isolated as an oil (126 mg, $87 \%$ yield) after flash chromatography (petroleum ether/ethyl acetate $=60 / 1) .{ }^{1} \mathrm{H}$ NMR $\left(400 \mathrm{MHz}, \mathrm{CDCl}_{3}\right)$ $\delta / \mathrm{ppm}=7.31-7.20(\mathrm{~m}, 5 \mathrm{H}), 4.30(\mathrm{dd}, J=8.3,4.7 \mathrm{~Hz}, 1 \mathrm{H}), 3.34-3.14(\mathrm{~m}, 4 \mathrm{H}), 1.58$ $-1.48(\mathrm{~m}, 2 \mathrm{H}), 0.86(\mathrm{t}, J=7.4 \mathrm{~Hz}, 3 \mathrm{H}) .{ }^{13} \mathrm{C} \mathrm{NMR}\left(100 \mathrm{MHz}, \mathrm{CDCl}_{3}\right) \delta / \mathrm{ppm}=139.5$, $127.6,127.2,125.5,80.9,70.3,21.9,9.9,9.8$. Spectral data are in good agreement with literature values. ${ }^{19}$

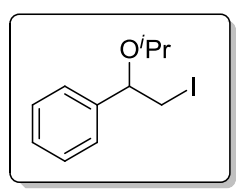

(2-Iodo-1-isopropoxyethyl)benzene (9j). Compound $\mathbf{9 j}$ was prepared according to the general procedure and isolated as an oil (117 $\mathrm{mg}, 81 \%$ yield) after flash chromatography (petroleum ether/ethyl acetate $=40 / 1) .{ }^{1} \mathrm{H} \mathrm{NMR}\left(400 \mathrm{MHz}, \mathrm{CDCl}_{3}\right)$ 
$\delta / \mathrm{ppm}=7.30-7.19(\mathrm{~m}, 5 \mathrm{H}), 4.28(\mathrm{dd}, J=8.4,4.5 \mathrm{~Hz}, 1 \mathrm{H}), 3.30-3.19(\mathrm{~m}, 2 \mathrm{H}), 3.04$ (dt, $J=15.4,8.9 \mathrm{~Hz}, 2 \mathrm{H}), 1.82(\mathrm{dp}, J=13.3,6.6 \mathrm{~Hz}, 1 \mathrm{H}), 0.85(\mathrm{dd}, J=11.3,6.7 \mathrm{~Hz}$, $6 \mathrm{H}) .{ }^{13} \mathrm{C} \mathrm{NMR}\left(100 \mathrm{MHz}, \mathrm{CDCl}_{3}\right) \delta / \mathrm{ppm}=139.5,127.6,127.2,125.4,81.0,75.4,27.6$, $18.5,18.5,9.9$. Spectral data are in good agreement with literature values. ${ }^{16}$

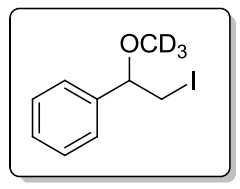

(2-Iodo-1-trideuteriomethoxyethyl)benzene (9k). Compound 9k was prepared according to the general procedure and isolated as an oil (109 $\mathrm{mg}, 82 \%$ yield) after flash chromatography (petroleum ether/ethyl acetate $=90 / 1) .{ }^{1} \mathrm{H}$ NMR $(400 \mathrm{MHz}$, $\left.\mathrm{CDCl}_{3}\right) \delta / \mathrm{ppm}=7.52-7.05(\mathrm{~m}, 1 \mathrm{H}), 4.22(\mathrm{dd}, J=7.8,5.0 \mathrm{~Hz}, 1 \mathrm{H}), 3.31-3.23(\mathrm{~m}$, $2 \mathrm{H}) .{ }^{13} \mathrm{C} \mathrm{NMR}\left(100 \mathrm{MHz}, \mathrm{CDCl}_{3}\right) \delta / \mathrm{ppm}=138.7,127.7,127.4,125.5,82.4,55.6-55.2$ (m), 9.5. Spectral data are in good agreement with literature values. ${ }^{20}$

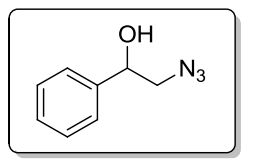

2-Azido-1-phenylethanol (10a). Following the general procedure for iodohydrins preparation using styrene ( $0.5 \mathrm{mmol}, 1$ equiv.), $\mathrm{I}_{2} \mathrm{O}_{5}(0.55 \mathrm{mmol}, 1.1$ equiv.) and LiI, ( $0.55 \mathrm{mmol}, 1.1$ equiv.) and usual work, the resulting residues were treated with $\mathrm{NaN}_{3}$ ( $1 \mathrm{mmol}, 2$ equiv.) in DMF $(2 \mathrm{~mL})$ and stirred at $70{ }^{\circ} \mathrm{C}$ until the completion of the reaction. The usual workup and purification by silica gel column chromatography afforded 10a in $89 \%$ isolated yield. ${ }^{1} \mathrm{H}$ NMR $\left(400 \mathrm{MHz}, \mathrm{CDCl}_{3}\right) \delta / \mathrm{ppm}=7.74-6.50$ $(\mathrm{m}, 5 \mathrm{H}), 4.69(\mathrm{dd}, J=7.5,4.2 \mathrm{~Hz}, 1 \mathrm{H}), 3.31(\mathrm{dd}, J=12.6,7.9 \mathrm{~Hz}, 1 \mathrm{H}), 3.25(\mathrm{dd}, J=$ 12.6, $4.2 \mathrm{~Hz}, 1 \mathrm{H}), 3.05$ (s, 1H). ${ }^{13} \mathrm{C}$ NMR $\left(100 \mathrm{MHz}, \mathrm{CDCl}_{3}\right) \delta / \mathrm{ppm}=140.7,128.7$, $128.4,126.0,73.4,57.9$. Spectral data are in good agreement with literature values. ${ }^{21}$<smiles>C1CC2CCCC(C1)O2</smiles>

5-Benzyldihydrofuran-2(3H)-one (10b). Following the general procedure for iodohydrins preparation using pent-4-enoic acid $5 \mathbf{a}(0.5 \mathrm{mmol}, 1$ equiv. $), \mathrm{I}_{2} \mathrm{O}_{5}(0.55$ mmol, 1.1 equiv.) and LiI ( $0.55 \mathrm{mmol}, 1.1$ equiv.) and usual work, the resulting residues $6 \mathbf{a}$ were treated with $\mathrm{NiBr}_{2}$ diglyme ( $0.05 \mathrm{mmol}, 0.1$ equiv.), 2,2'-bipyridine (0.06 mmol, 0.12 equiv.), Mn ( $1.5 \mathrm{mmol}, 3$ equiv.) and iodobenzene ( $0.5 \mathrm{mmol}, 1$ equiv.), followed by DMA (1 mL). The vial was then evacuated and backfilled with nitrogen three times. The reaction mixture was then stirred at room temperature overnight. The reaction mixture was diluted with EtOAc $(4 \mathrm{~mL})$ and water $(3 \mathrm{~mL})$ was then added. The organic layer was separated, and the aqueous layer was extracted with EtOAc $(2 \times 4 \mathrm{~mL})$. The combined organic layers were washed with water $(2 \times 4$ $\mathrm{mL}$ ), dried over $\mathrm{Na}_{2} \mathrm{SO}_{4}$ and concentrated under reduced pressure to give the crude product, which was purified by column chromatography (petroleum ether/ethyl acetate $=6 / 1$ ) to afforded $\mathbf{1 0 b}$ in $62 \%$ isolated yield (oil, $55 \mathrm{mg}$ ). ${ }^{1} \mathrm{H}$ NMR (400 MHz, 
$\left.\mathrm{CDCl}_{3}\right) \delta / \mathrm{ppm}=7.53-6.48(\mathrm{~m}, 5 \mathrm{H}), 4.66(\mathrm{dq}, J=7.5,6.3 \mathrm{~Hz}, 1 \mathrm{H}), 2.99(\mathrm{dd}, J=14.0$, $6.1 \mathrm{~Hz}, 1 \mathrm{H}), 2.85(\mathrm{dd}, J=14.0,6.2 \mathrm{~Hz}, 1 \mathrm{H}), 2.48-2.24(\mathrm{~m}, 2 \mathrm{H}), 2.23-2.14(\mathrm{~m}, 1 \mathrm{H})$, $1.95-1.84(\mathrm{~m}, 1 \mathrm{H}) .{ }^{13} \mathrm{C} \mathrm{NMR}\left(100 \mathrm{MHz}, \mathrm{CDCl}_{3}\right) \delta / \mathrm{ppm}=176.1,134.9,128.4,127.6$, 125.9, 79.8, 40.3, 27.6, 26.1. Spectral data are in good agreement with literature values. ${ }^{9}$

\section{ORTEP drawing of 8d}

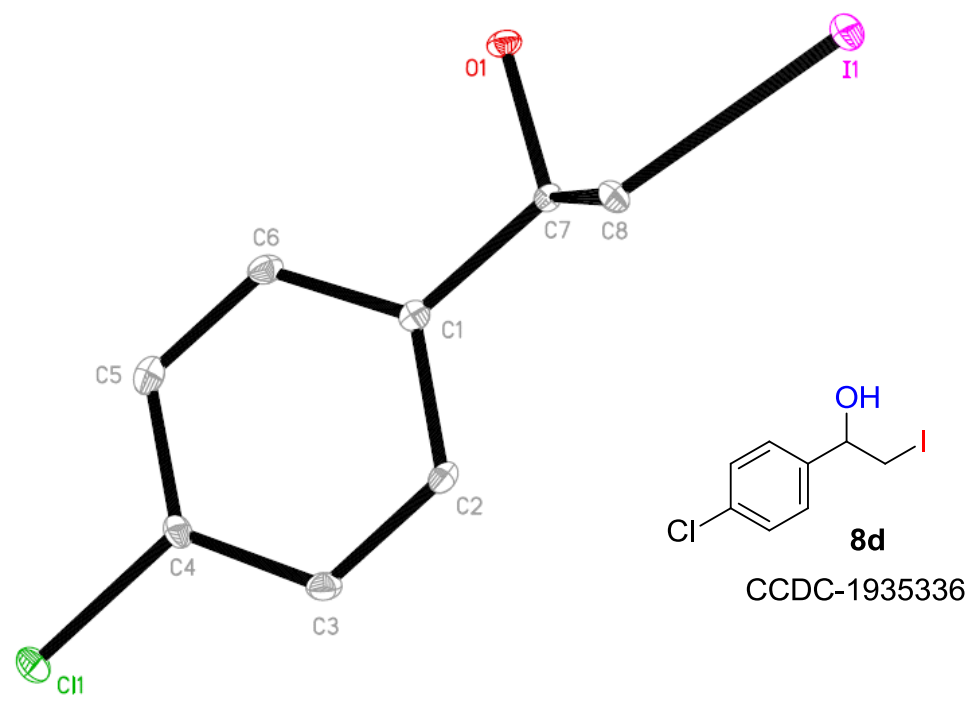

Figure S2. ORTEP drawing of 8d. Hydrogen atoms were omitted for clarity.

Table S1 Crystal data and structure refinement for $\mathbf{8 d}$.

\begin{tabular}{|c|c|}
\hline Empirical formula & $\mathrm{C}_{8} \mathrm{H}_{8} \mathrm{ClIO}$ \\
\hline Formula weight & 282.49 \\
\hline Temperature/K & $100.01(10)$ \\
\hline Crystal system & monoclinic \\
\hline Space group & $\mathrm{P} 2_{1} / \mathrm{n}$ \\
\hline $\mathrm{a} / \AA$ & $13.0502(7)$ \\
\hline $\mathrm{b} / \AA ̊ \AA$ & $4.5002(2)$ \\
\hline $\mathrm{c} / \AA ̊$ & $15.3524(8)$ \\
\hline $\mathrm{a} /{ }^{\circ}$ & 90 \\
\hline$\beta /{ }^{\circ}$ & $99.661(5)$ \\
\hline$y /{ }^{\circ}$ & 90 \\
\hline Volume $/ \AA^{3}$ & $888.84(8)$ \\
\hline $\mathrm{Z}$ & 4 \\
\hline$\rho c a l c g / \mathrm{cm}^{3}$ & 2.111 \\
\hline$\mu / \mathrm{mm}^{-1}$ & 3.842 \\
\hline $\mathrm{F}(000)$ & 536.0 \\
\hline Crystal size $/ \mathrm{mm}^{3}$ & $0.12 \times 0.11 \times 0.09$ \\
\hline Radiation & $\operatorname{MoKa}(\lambda=0.71073)$ \\
\hline
\end{tabular}




\begin{tabular}{|c|c|}
\hline $2 \mathrm{~T}$ range for data collection $/{ }^{\circ}$ & 4.486 to 49.944 \\
\hline Index ranges & $-13=\mathrm{h}=15,-5=\mathrm{k}=5,-16=1=18$ \\
\hline Reflections collected & 5115 \\
\hline Independent reflections & $1565\left[R_{\text {int }}=0.0244, R_{\text {sigma }}=0.0244\right]$ \\
\hline Data/restraints/parameters & $1565 / 0 / 104$ \\
\hline Goodness-of-fit on $\mathrm{F}^{2}$ & 1.077 \\
\hline Final $\mathrm{R}$ indexes $[\mathrm{I}>=2 \mathrm{~s}(\mathrm{I})]$ & $\mathrm{R} 1=0.0189, \mathrm{wR} 2=0.0455$ \\
\hline Final R indexes [all data] & $\mathrm{R} 1=0.0198, \mathrm{wR} 2=0.0461$ \\
\hline Largest diff. peak/hole / e $\AA^{-3}$ & $0.38 /-0.60$ \\
\hline
\end{tabular}

Table S2 Fractional Atomic Coordinates $\left(\times 10^{4}\right)$ and Equivalent Isotropic Displacement Parameters $\left(\AA^{2} \times 10^{3}\right)$ for $\mathbf{8 d}$.

\begin{tabular}{|c|c|c|c|c|}
\hline Atom & $\mathrm{x}$ & $\mathrm{y}$ & $\mathrm{z}$ & $\mathrm{U}(\mathrm{eq})$ \\
\hline $\mathrm{I} 1$ & $6622.7(2)$ & $8940.5(4)$ & $5107.9(2)$ & $12.97(9)$ \\
\hline C11 & $3481.8(6)$ & $1118.9(15)$ & \multicolumn{2}{|c|}{$9171.0(5) 17.07(17)$} \\
\hline O1 & $6889.8(15)$ & $9376(4)$ & $7343.4(13)$ & $12.8(4)$ \\
\hline C1 & $5283(2)$ & $6745(6)$ & $7508.9(18)$ & $10.5(6)$ \\
\hline C7 & $5875(2)$ & $8508(6)$ & $6914.2(18)$ & $11.0(6)$ \\
\hline C8 & $5929(2)$ & $6643(6)$ & $6094.9(18)$ & $12.3(6)$ \\
\hline C2 & $4244(2)$ & $5995(6)$ & $7206.4(19)$ & $13.6(6)$ \\
\hline C6 & $5749(2)$ & $5743(6)$ & $8340.9(18)$ & $13.9(6)$ \\
\hline C3 & $3690(2)$ & $4280(6)$ & $7711.7(19)$ & $13.9(6)$ \\
\hline C5 & $5206(2)$ & $4015(6)$ & $8853.1(19)$ & $15.9(7)$ \\
\hline C4 & $4183(2)$ & $3288(6)$ & $8531.5(18)$ & $12.4(6)$ \\
\hline
\end{tabular}

Table S3 Anisotropic Displacement Parameters $\left(\AA^{2} \times 10^{3}\right)$ for $8 d$.

\begin{tabular}{|c|c|c|c|c|c|c|}
\hline Atom & $\mathrm{U} 11$ & $\mathrm{U} 22$ & $\mathrm{U} 33$ & $\mathrm{U} 23$ & $\mathrm{U} 13$ & $\mathrm{U} 12$ \\
\hline I1 & $13.45(13)$ & $15.17(13)$ & $11.03(12)$ & $0.69(7)$ & $4.20(8)$ & $-0.45(7)$ \\
\hline C11 & $16.6(4)$ & $20.1(4)$ & $16.0(4)$ & $4.4(3)$ & $7.2(3)$ & $-2.0(3)$ \\
\hline O1 & $8.7(10)$ & $10.0(10)$ & $18.7(11)$ & $0.0(9)$ & $-0.2(8)$ & $-0.9(9)$ \\
\hline C1 & $11.2(15)$ & $8.4(13)$ & $11.8(14)$ & $-2.1(12)$ & $1.8(11)$ & $1.7(11)$ \\
\hline C7 & $9.2(15)$ & $11.5(14)$ & $12.0(14)$ & $2.0(11)$ & $1.1(11)$ & $1.4(11)$ \\
\hline C8 & $12.1(15)$ & $11.5(14)$ & $13.5(14)$ & $2.8(11)$ & $2.4(12)$ & $-3.1(11)$ \\
\hline C2 & $11.4(16)$ & $14.9(15)$ & $13.2(15)$ & $2.2(11)$ & $-1.7(12)$ & $1.3(11)$ \\
\hline C6 & $10.5(15)$ & $19.3(16)$ & $11.4(14)$ & $-1.8(12)$ & $0.5(12)$ & $-0.6(11)$ \\
\hline C3 & $9.0(15)$ & $16.9(15)$ & $15.9(15)$ & $-0.2(12)$ & $2.5(12)$ & $-0.8(11)$ \\
\hline C5 & $17.3(17)$ & $17.2(16)$ & $12.5(15)$ & $1.3(12)$ & $0.4(13)$ & $3.5(11)$ \\
\hline C4 & $14.3(15)$ & $11.9(14)$ & $12.3(14)$ & $1.8(12)$ & $5.7(12)$ & $0.9(11)$ \\
\hline
\end{tabular}


Table S4 Bond Lengths for $8 \mathbf{d}$.

\begin{tabular}{|c|c|c|c|c|c|}
\hline Aton & n Atom & Length/Å & Atom & Atom & Length/Å \\
\hline I1 & $\mathrm{C} 8$ & $2.155(3)$ & C7 & $\mathrm{C} 8$ & $1.524(4)$ \\
\hline $\mathrm{Cl1}$ & $\mathrm{C} 4$ & $1.748(3)$ & $\mathrm{C} 2$ & $\mathrm{C} 3$ & $1.382(4)$ \\
\hline $\mathrm{O} 1$ & C7 & $1.431(3)$ & C6 & C5 & $1.382(4)$ \\
\hline $\mathrm{C} 1$ & $\mathrm{C} 7$ & $1.516(4)$ & C3 & $\mathrm{C} 4$ & $1.388(4)$ \\
\hline $\mathrm{C} 1$ & $\mathrm{C} 2$ & $1.399(4)$ & C5 & $\mathrm{C} 4$ & $1.383(4)$ \\
\hline $\mathrm{C} 1$ & C6 & $1.394(4)$ & & & \\
\hline
\end{tabular}

Table S5 Bond Angles for 8d

\begin{tabular}{|c|c|c|c|c|c|c|c|}
\hline Atom & Atom & Atom & Angle ${ }^{\circ}$ & Atom & Atom & Atom & Angle $/^{\circ}$ \\
\hline $\mathrm{C} 2$ & $\mathrm{C} 1$ & $\mathrm{C} 7$ & 119.3(2) & $\mathrm{C} 3$ & $\mathrm{C} 2$ & $\mathrm{C} 1$ & 121.2(3) \\
\hline C6 & $\mathrm{C} 1$ & $\mathrm{C} 7$ & $122.3(2)$ & $\mathrm{C} 5$ & C6 & $\mathrm{C} 1$ & 121.2(3) \\
\hline C6 & $\mathrm{C} 1$ & $\mathrm{C} 2$ & $118.3(3)$ & $\mathrm{C} 2$ & $\mathrm{C} 3$ & $\mathrm{C} 4$ & $118.8(3)$ \\
\hline $\mathrm{O} 1$ & $\mathrm{C} 7$ & $\mathrm{C} 1$ & $113.0(2)$ & $\mathrm{C} 4$ & $\mathrm{C} 5$ & C6 & 119.1(3) \\
\hline $\mathrm{O} 1$ & $\mathrm{C} 7$ & $\mathrm{C} 8$ & $111.3(2)$ & $\mathrm{C} 3$ & $\mathrm{C} 4$ & $\mathrm{Cl} 1$ & $118.8(2)$ \\
\hline $\mathrm{C} 1$ & $\mathrm{C} 7$ & $\mathrm{C} 8$ & $107.9(2)$ & $\mathrm{C} 3$ & $\mathrm{C} 4$ & $\mathrm{C} 5$ & 121.4(3) \\
\hline $\mathrm{C} 7$ & $\mathrm{C} 8$ & I1 & $113.51(18)$ & $\mathrm{C} 5$ & $\mathrm{C} 4$ & $\mathrm{Cl} 1$ & $119.8(2)$ \\
\hline
\end{tabular}

Table S6 Hydrogen Atom Coordinates $\left(\AA \times 10^{4}\right)$ and Isotropic Displacement Parameters $\left(\AA^{2} \times 10^{3}\right)$ for $\mathbf{8 d}$.

\begin{tabular}{|c|c|c|c|c|}
\hline Atom & $x$ & $y$ & $z$ & $U(e q)$ \\
\hline H8A & 5231.3 & 6035.03 & 5836.01 & 15 \\
\hline H8B & 6328.35 & 4859.28 & 6270.64 & 15 \\
\hline H2 & 3920.76 & 6662.37 & 6655.13 & 16 \\
\hline H6 & 6435.86 & 6245.16 & 8555.09 & 17 \\
\hline H3 & 2998.14 & 3800.2 & 7506.16 & 17 \\
\hline H5 & 5524.84 & 3350.38 & 9406.25 & 19 \\
\hline H1 & $7260(20)$ & $7880(80)$ & $7420(20)$ & $18(9)$ \\
\hline
\end{tabular}

\section{References}

1. A. Jaganathan, A. Garzan, D. C. Whitehead, R. J. Staples and B. Borhan, Angew. Chem., Int. Ed., 2011, 50, 2593.

2. G.-Q. Liu, Z.-Y. Ding, L. Zhang, T.-T. Li, L. Li, L. Duan and Y.-M. Li, Adv. Synth. Catal., 2014, 356, 2303.

3. W. Yi, X.-X. Fang, Q.-Y. Liu and G.-Q. Liu, Eur. J. Org. Chem., 2018, 2018, 6671.

4. S. D. Karyakarte, T. P. Smith and S. R. Chemler, J. Org. Chem., 2012, 77, 7755.

5. X.-W. Zhang, Z.-F. Xiao, M.-M. Wang, Y.-J. Zhuang and Y.-B. Kang, Org. Biomol. Chem., 2016, 14, 7275.

6. G.-Q. Liu, C.-H. Yang and Y.-M. Li, J. Org. Chem., 2015, 80, 11339. 
7. K. Nagaraju, N. Rajesh and P. R. Krishna, Synth. Commun., 2018, 48, 1001.

8. G.-Q. Liu and Y.-M. Li, J. Org. Chem., 2014, 79, 10094.

9. J. P. Ariyarathna, F. Wu, S. K. Colombo, C. M. Hillary and W. Li, Org. Lett., 2018, $20,6462$.

10. S. W. Park, S.-H. Kim, J. Song, G. Y. Park, D. Kim, T.-G. Nam and K. B. Hong, Beilstein J. Org. Chem., 2018, 14, 1028.

11. M. Smietana, V. Gouverneur and C. Mioskowski, Tetrahedron Lett., 2000, 41, 193.

12. G. Asensio, C. Andreu, C. Boix-Bernardini, R. Mello and M. E. Gonzalez-Nunez, Org. Lett., 1999, 1, 2125.

13. D. S. Rao, T. R. Reddy, K. Babachary and S. Kashyap, Org. Biomol. Chem., 2016, 14, 7529.

14. M. Jereb, M. Zupan and S. Stavber, Green Chem., 2005, 7, 100.

15. A. R. De Corso, B. Panunzi and M. Tingoli, Tetrahedron Lett., 2001, 42, 7245.

16. R. d. S. Ribeiro, P. M. Esteves and M. C. S. de Mattos, Tetrahedron Lett., 2007, 48, 8747.

17. N. Chakraborty, S. Santra, S. K. Kundu, A. Hajra, G. V. Zyryanov and A. Majee, RSC Adv, 2015, 5, 56780 .

18. N. Iranpoor and M. Shekarriz, Tetrahedron, 2000, 56, 5209.

19. A. Onoe, S. Uemura and M. Okano, Bull. Chem. Soc. Jpn., 1974, 47, 2818.

20. T. K. Achar, S. Maiti and P. Mal, Org. Biomol. Chem., 2016, 14, 4654.

21. T. R. Reddy, D. S. Rao and S. Kashyap, Chem. Commun. , 2019, 55, 2833.

\section{Copies of NMR spectra}
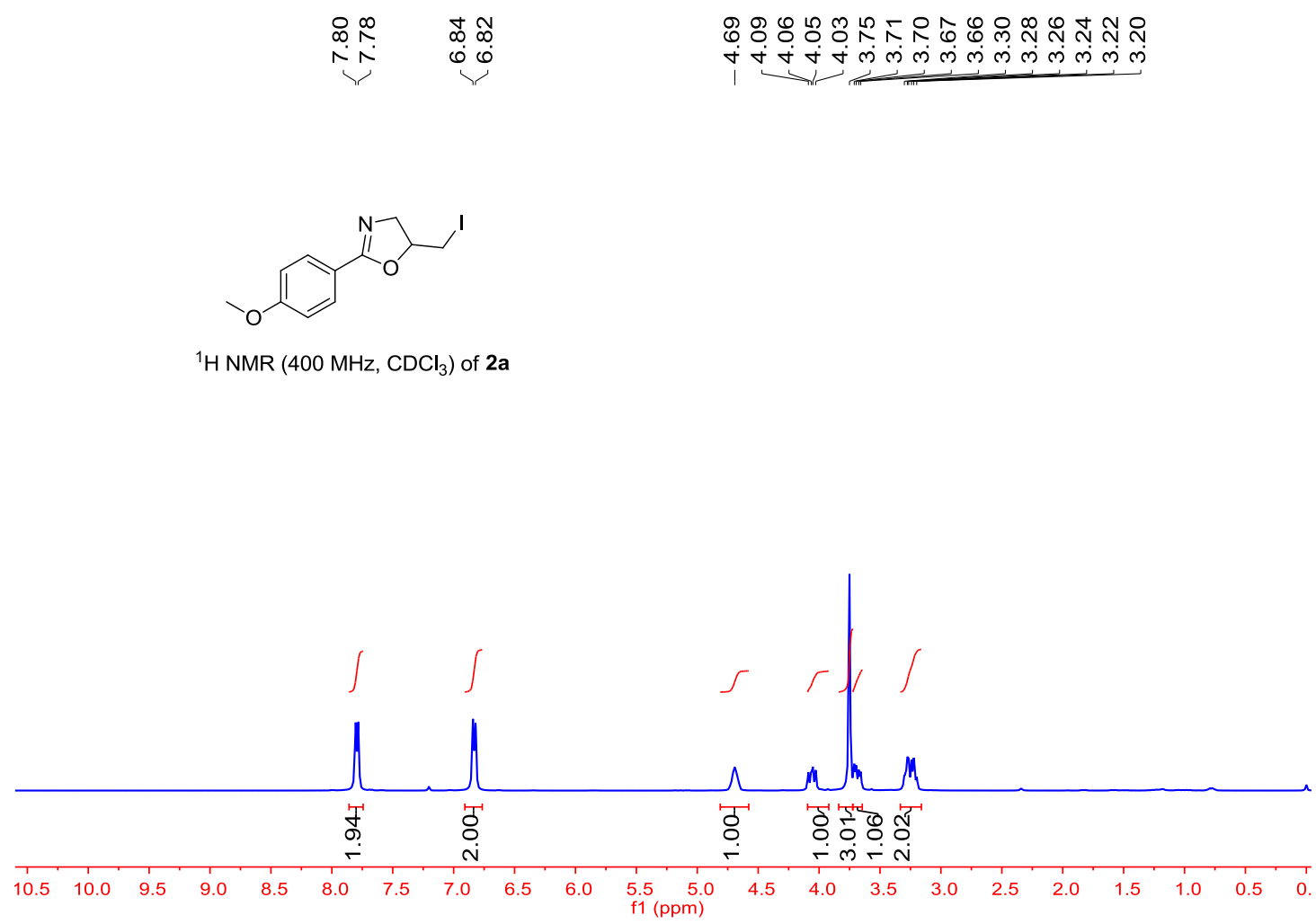

กับ

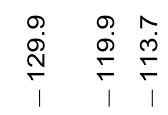

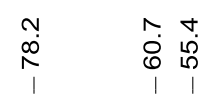

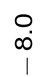

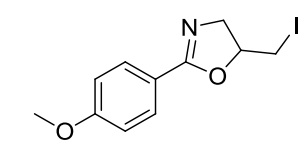

$\left.{ }^{13} \mathrm{C} \mathrm{NMR} \mathrm{(100} \mathrm{MHz,} \mathrm{CDCl}_{3}\right)$ of $2 \mathrm{a}$

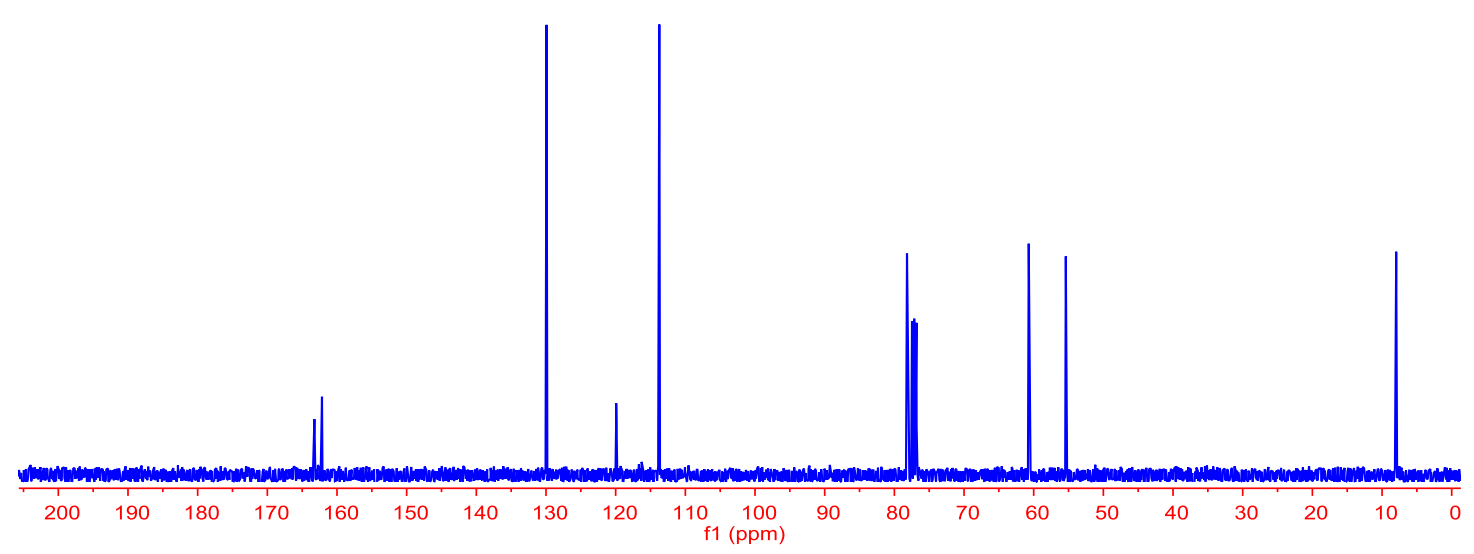

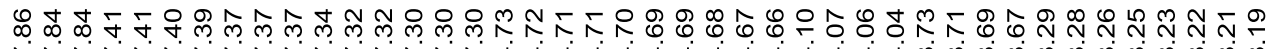

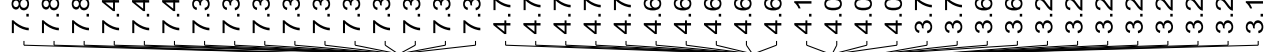

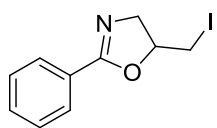

${ }^{1} \mathrm{H} \mathrm{NMR}\left(400 \mathrm{MHz}, \mathrm{CDCl}_{3}\right)$ of $\mathbf{2 b}$

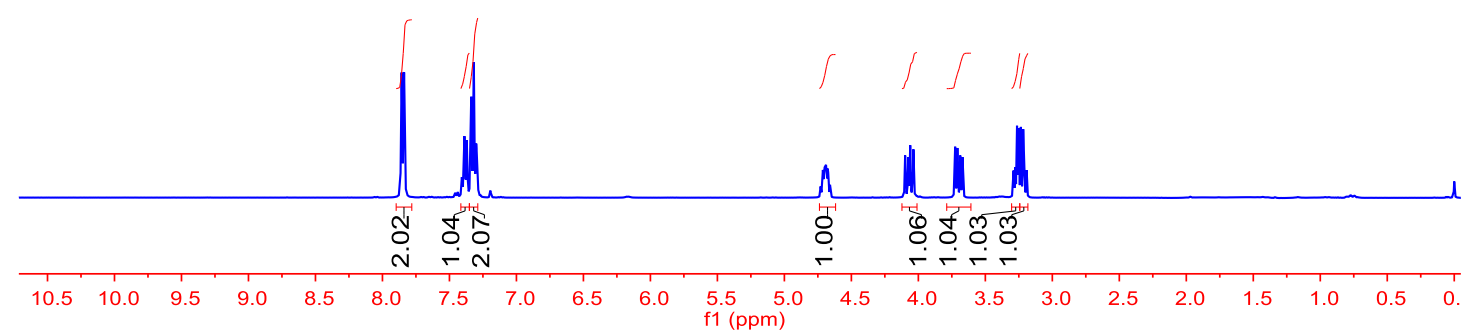




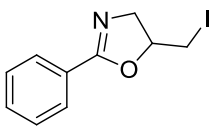

$\left.{ }^{13} \mathrm{C} \mathrm{NMR} \mathrm{(100} \mathrm{MHz,} \mathrm{CDCl}_{3}\right)$ of $\mathbf{2 b}$

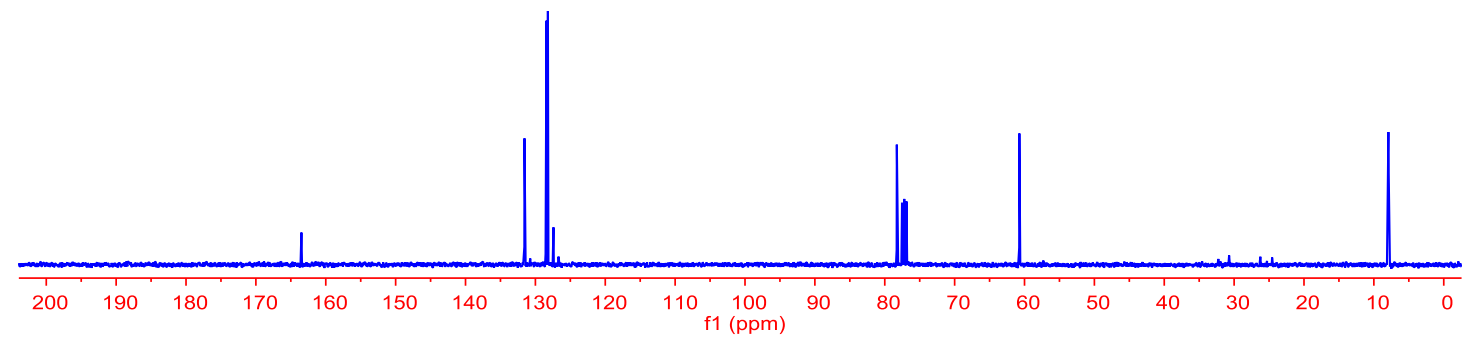

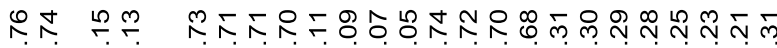

年

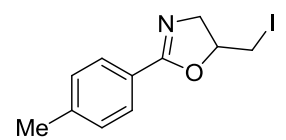

${ }^{1} \mathrm{H} \mathrm{NMR} \mathrm{(400} \mathrm{MHz,} \mathrm{CDCl}_{3}$ ) of $2 \mathrm{c}$

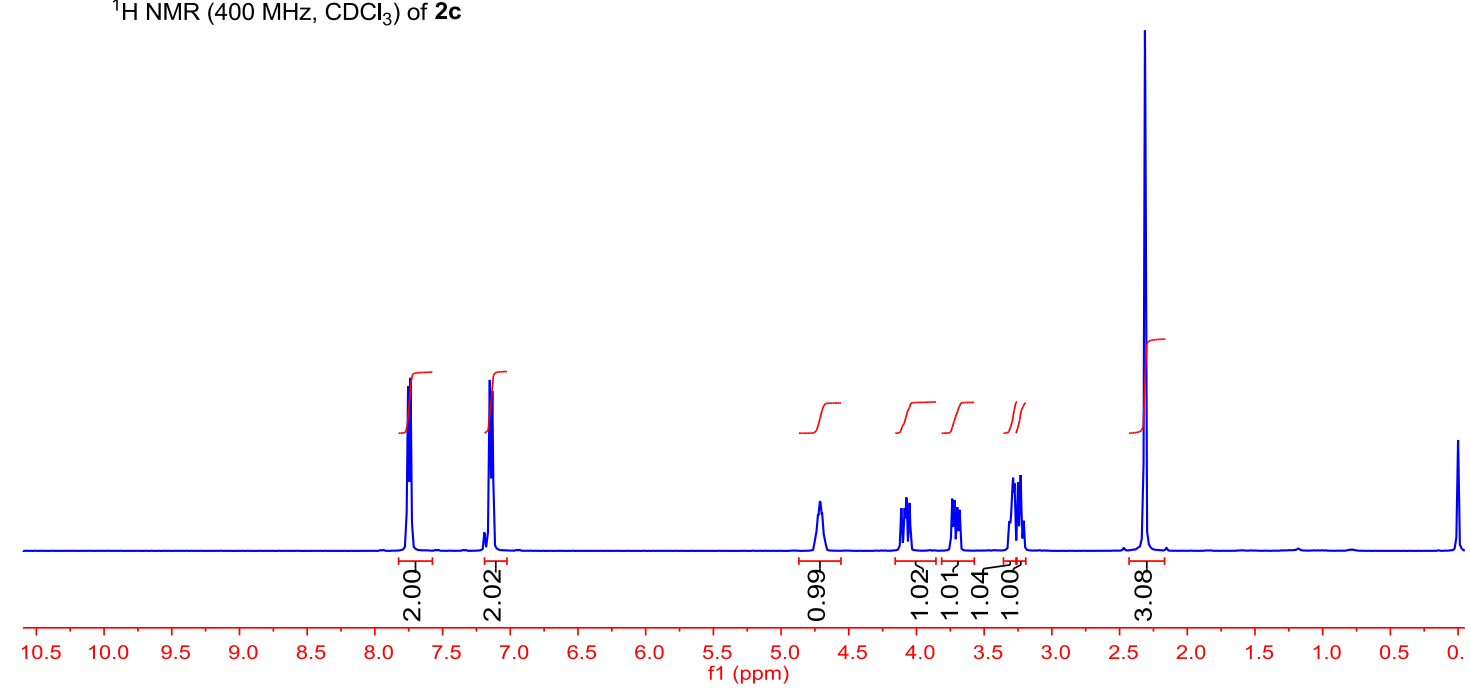




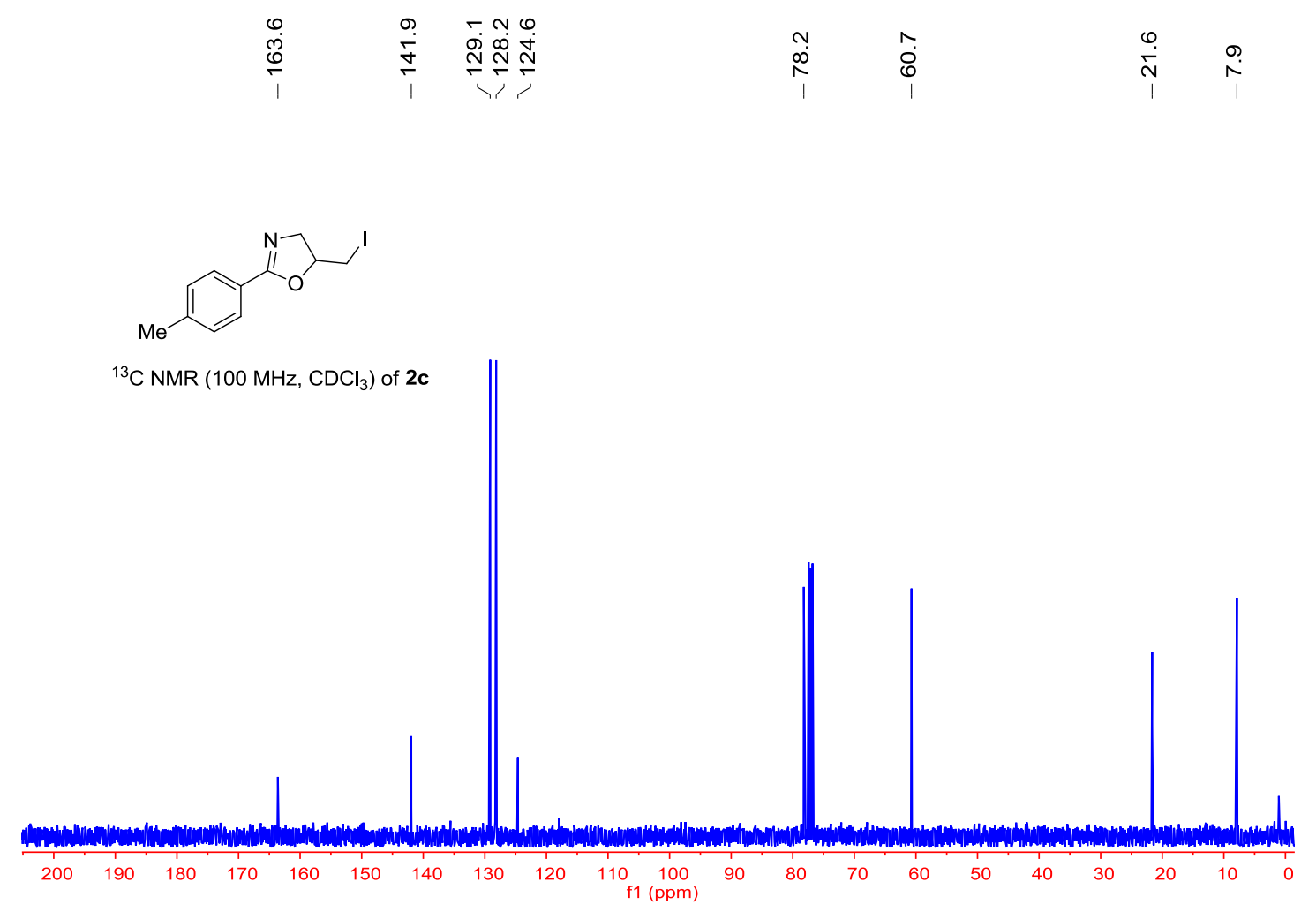

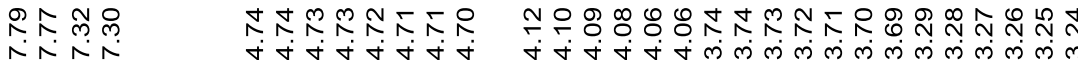

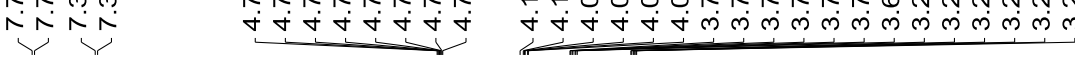

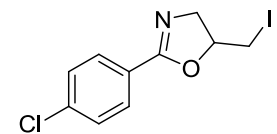

${ }^{1} \mathrm{H}$ NMR $\left(400 \mathrm{MHz}, \mathrm{CDCl}_{3}\right)$ of $\mathbf{2 d}$

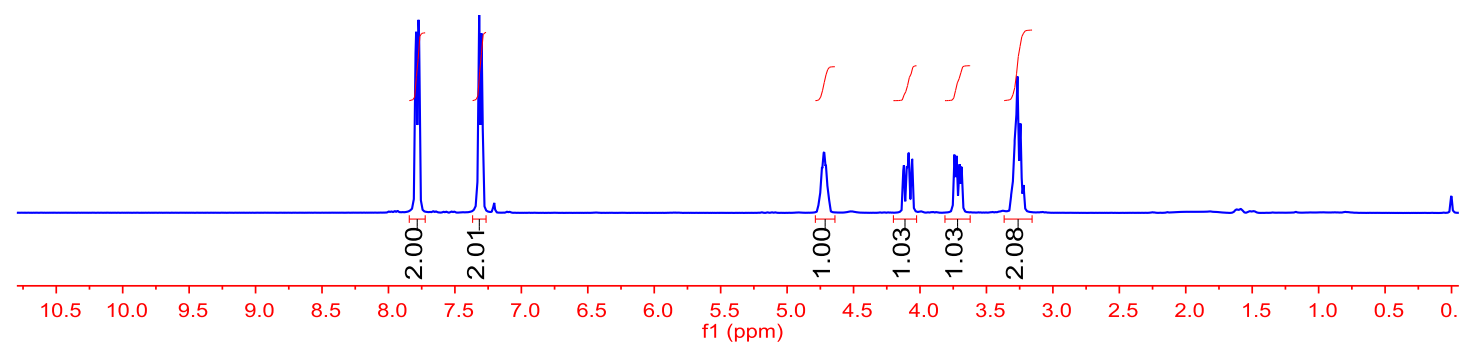




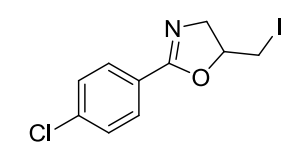

${ }^{13} \mathrm{C} \mathrm{NMR}\left(100 \mathrm{MHz}, \mathrm{CDCl}_{3}\right)$ of $\mathbf{2 d}$

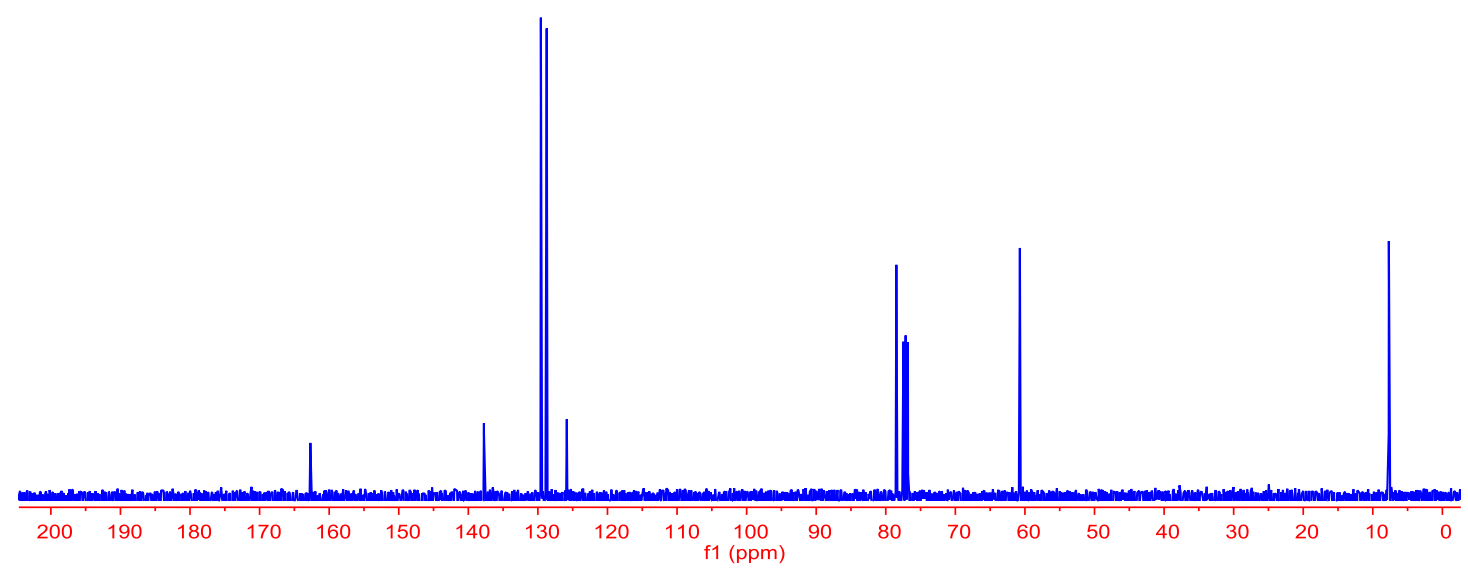

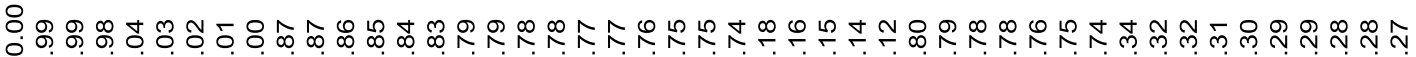

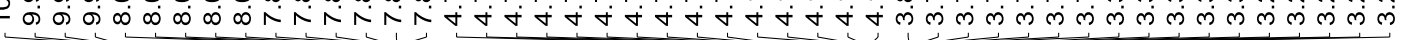

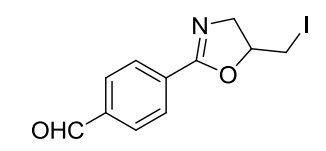

${ }^{1} \mathrm{H} \mathrm{NMR}\left(400 \mathrm{MHz}, \mathrm{CDCl}_{3}\right)$ of $2 \mathrm{e}$

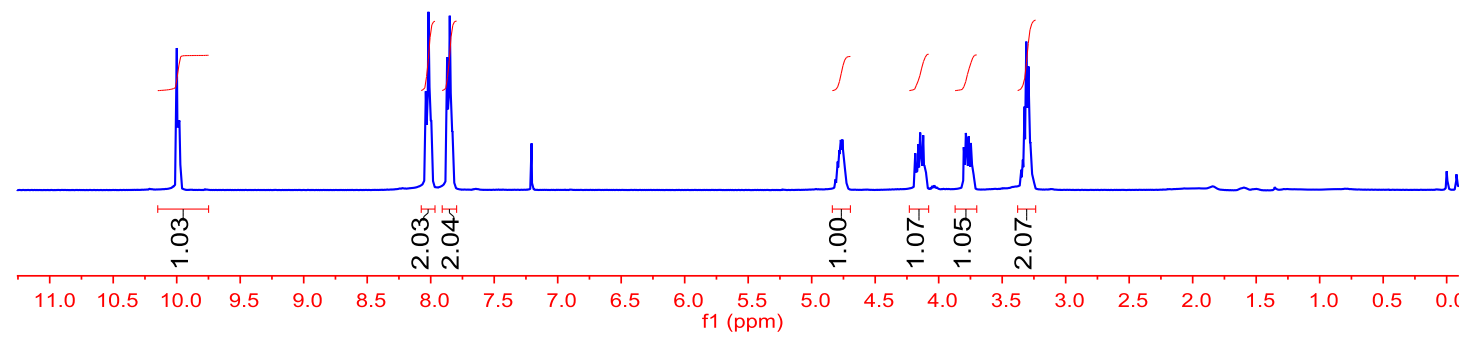




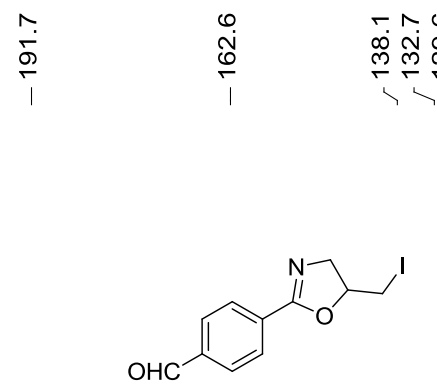

${ }^{13} \mathrm{C} \mathrm{NMR} \mathrm{(100} \mathrm{MHz,} \mathrm{CDCl}_{3}$ ) of $\mathbf{2 e}$

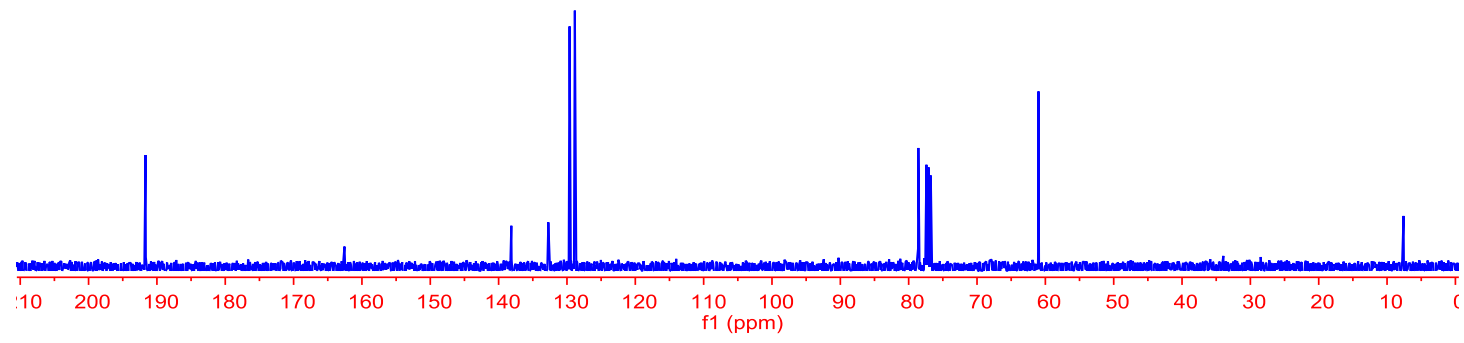

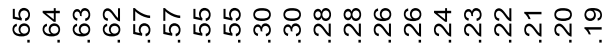

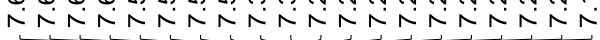

ธธ㇒ุำ मेंल लं लं

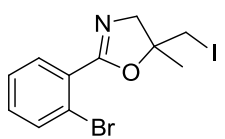

${ }^{1} \mathrm{H}$ NMR $\left(400 \mathrm{MHz}, \mathrm{CDCl}_{3}\right)$ of $\mathbf{2 f}$

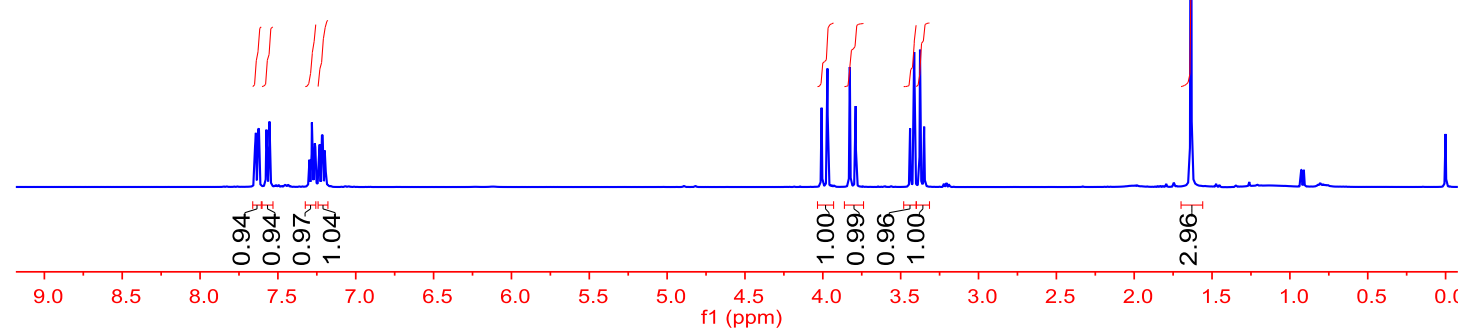




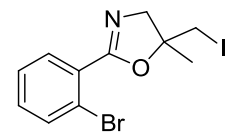

${ }^{13} \mathrm{C} \mathrm{NMR} \mathrm{(100} \mathrm{MHz,} \mathrm{CDCl}_{3}$ ) of $\mathbf{2 f}$

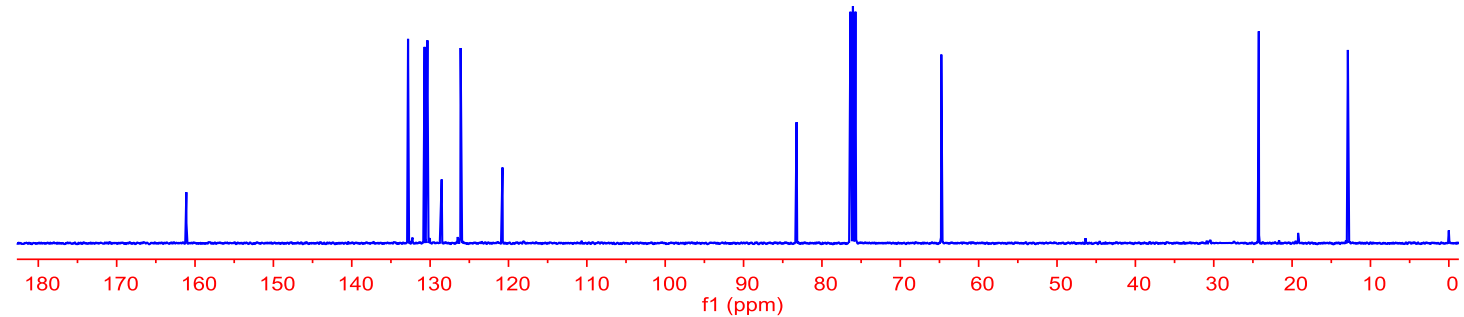

ำก윰ำ

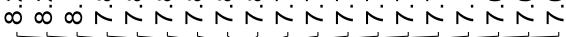

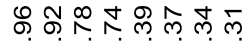
लंगुलंक्षिक

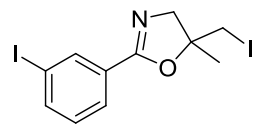

${ }^{1} \mathrm{H}$ NMR $\left(100 \mathrm{MHz}, \mathrm{CDCl}_{3}\right)$ of $\mathbf{2 g}$

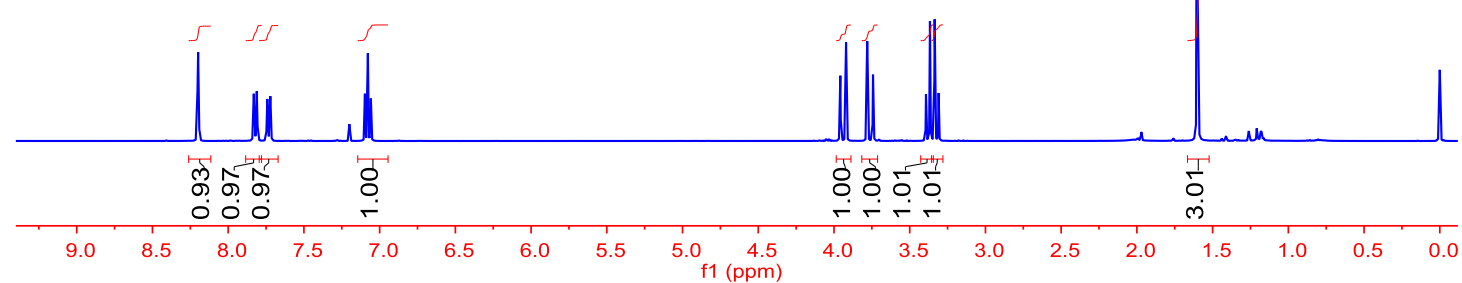




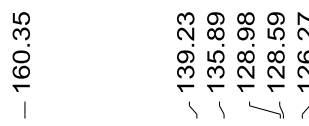

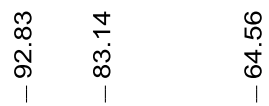

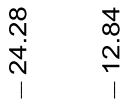

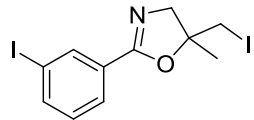

${ }^{13} \mathrm{C} \mathrm{NMR} \mathrm{(100} \mathrm{MHz,} \mathrm{CDCl}_{3}$ ) of $\mathbf{2 g}$

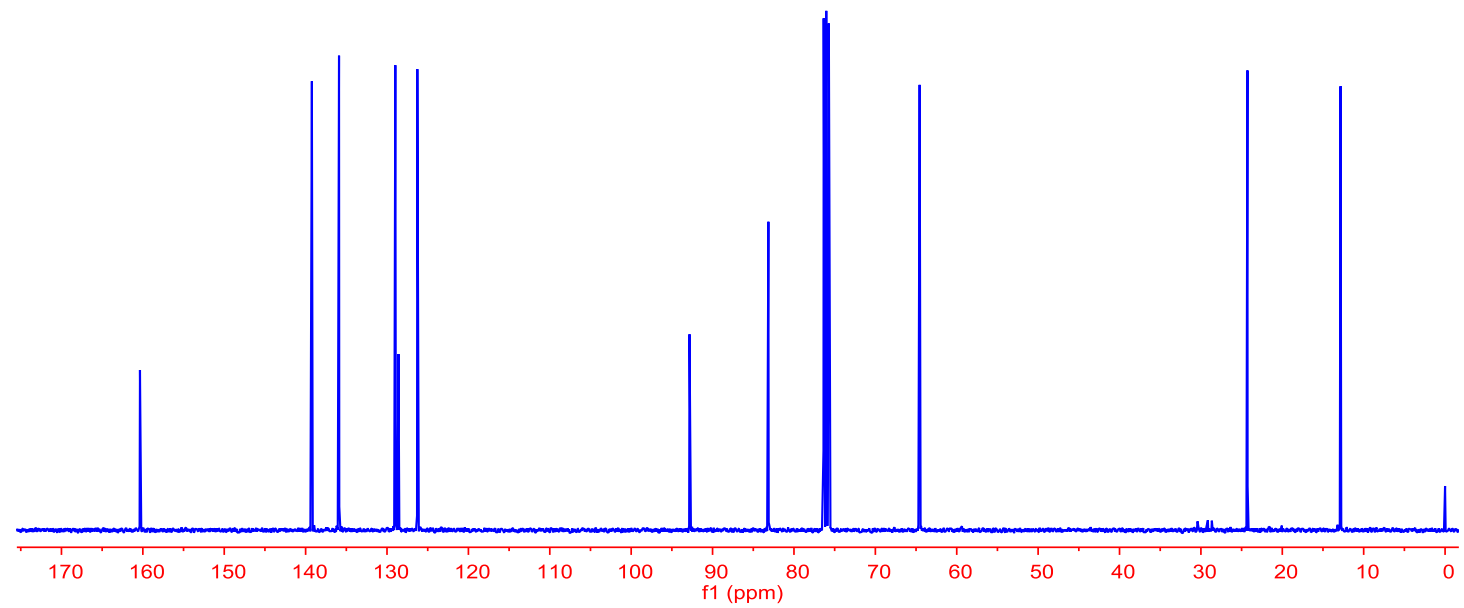

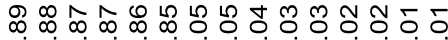

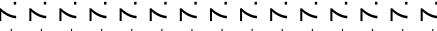

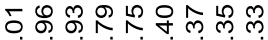

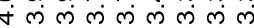

(

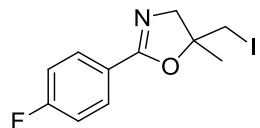

${ }^{1} \mathrm{H}$ NMR $\left(400 \mathrm{MHz}, \mathrm{CDCl}_{3}\right)$ of $\mathbf{2 h}$

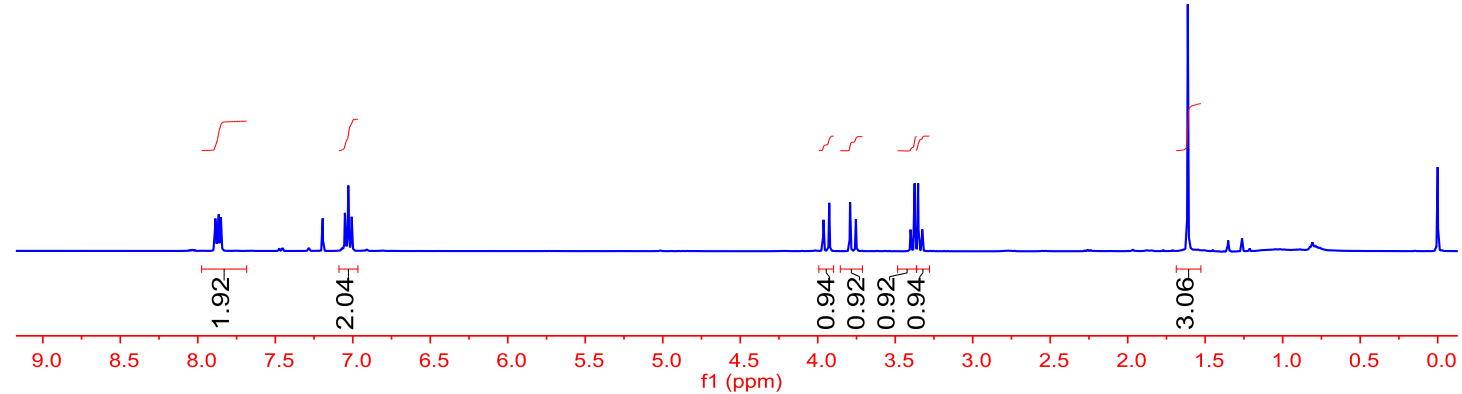




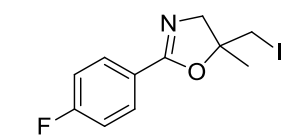

${ }^{19} \mathrm{~F} \mathrm{NMR} \mathrm{(376} \mathrm{MHz,} \mathrm{CDCl}_{3}$ ) of $\mathbf{2 h}$

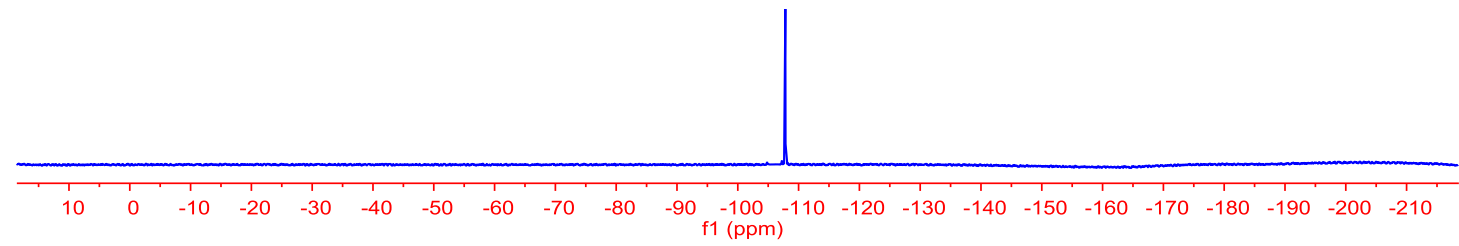

누윰

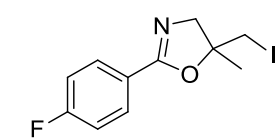

${ }^{13} \mathrm{C} \mathrm{NMR}\left(100 \mathrm{MHz}, \mathrm{CDCl}_{3}\right)$ of $\mathbf{2 h}$

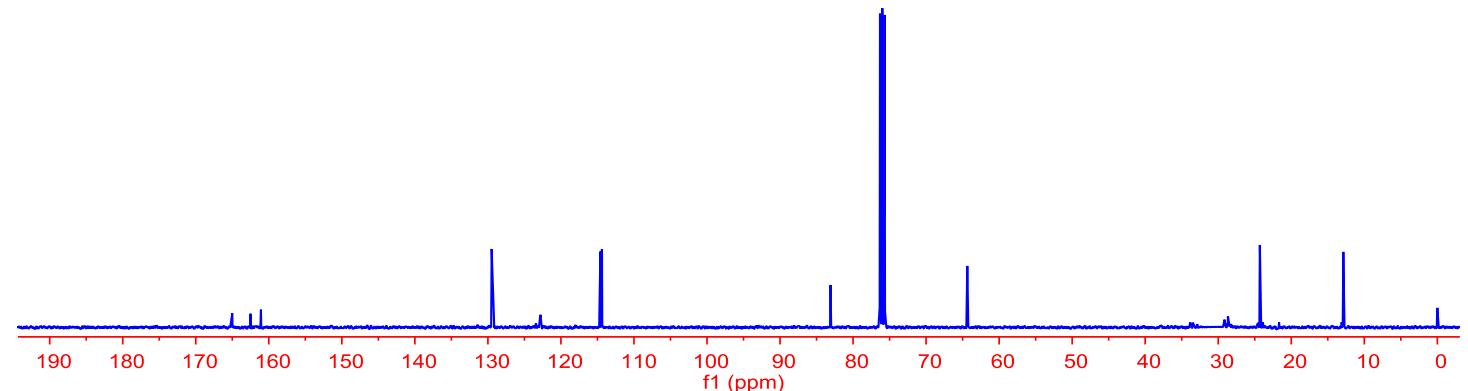




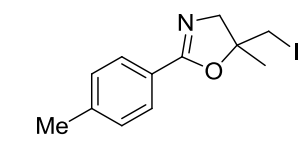

${ }^{1} \mathrm{H}$ NMR $\left(400 \mathrm{MHz}, \mathrm{CDCl}_{3}\right)$ of $\mathbf{2 i}$

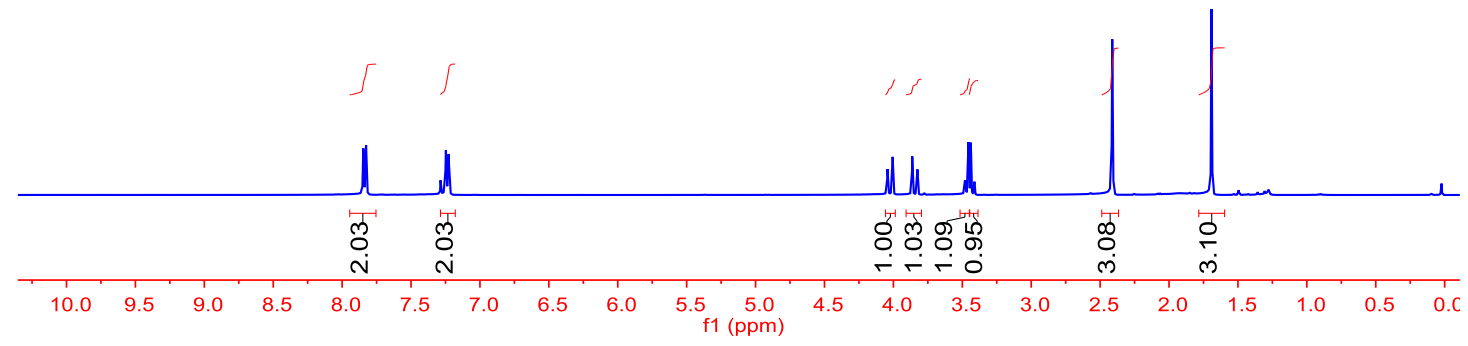

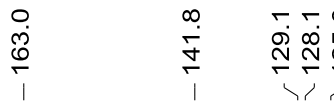

$\begin{array}{ll}0 & 10 \\ \infty & 1 \\ \infty & 0\end{array}$

ஸึ่

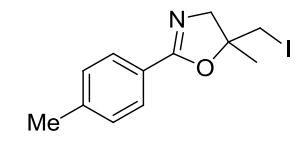

${ }^{13} \mathrm{C} \mathrm{NMR}\left(100 \mathrm{MHz}, \mathrm{CDCl}_{3}\right)$ of $\mathbf{2 i}$

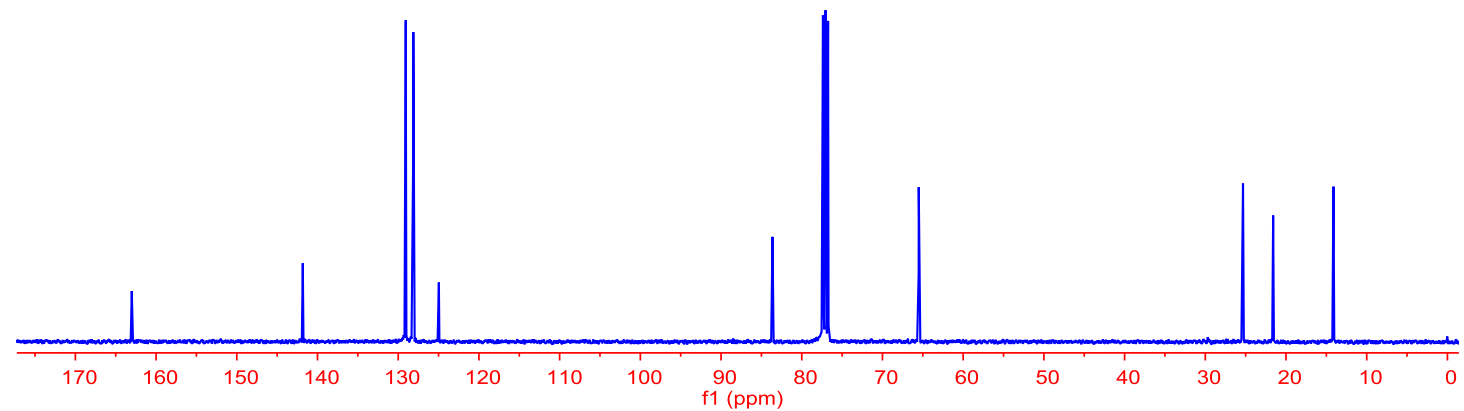




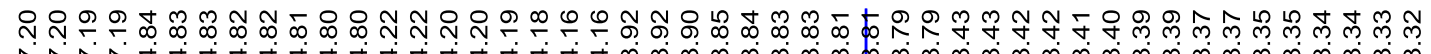

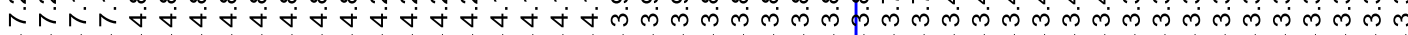

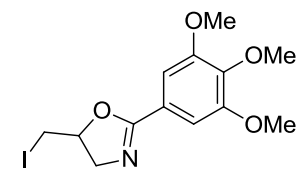

${ }^{1} \mathrm{H}$ NMR $\left(400 \mathrm{MHz}, \mathrm{CDCl}_{3}\right)$ of $\mathbf{2} \mathbf{j}$

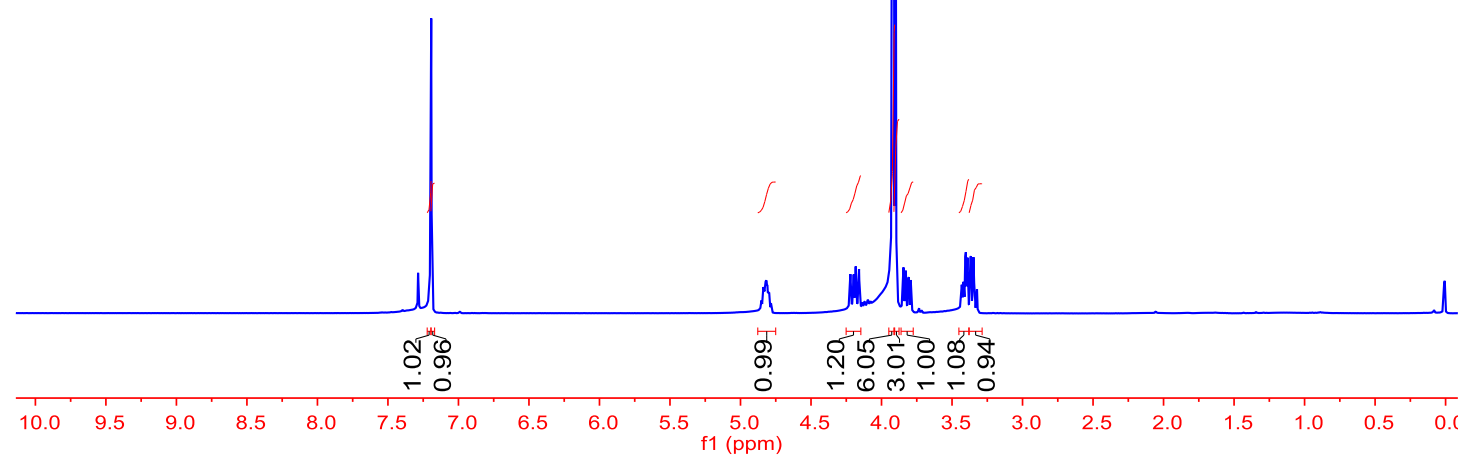

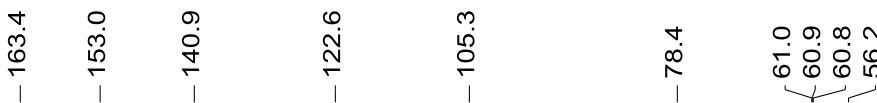

$\stackrel{\infty}{i}$

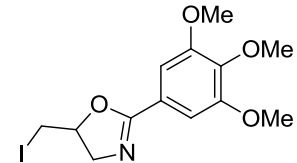

${ }^{13} \mathrm{C} \mathrm{NMR}\left(100 \mathrm{MHz}, \mathrm{CDCl}_{3}\right)$ of $2 \mathrm{j}$

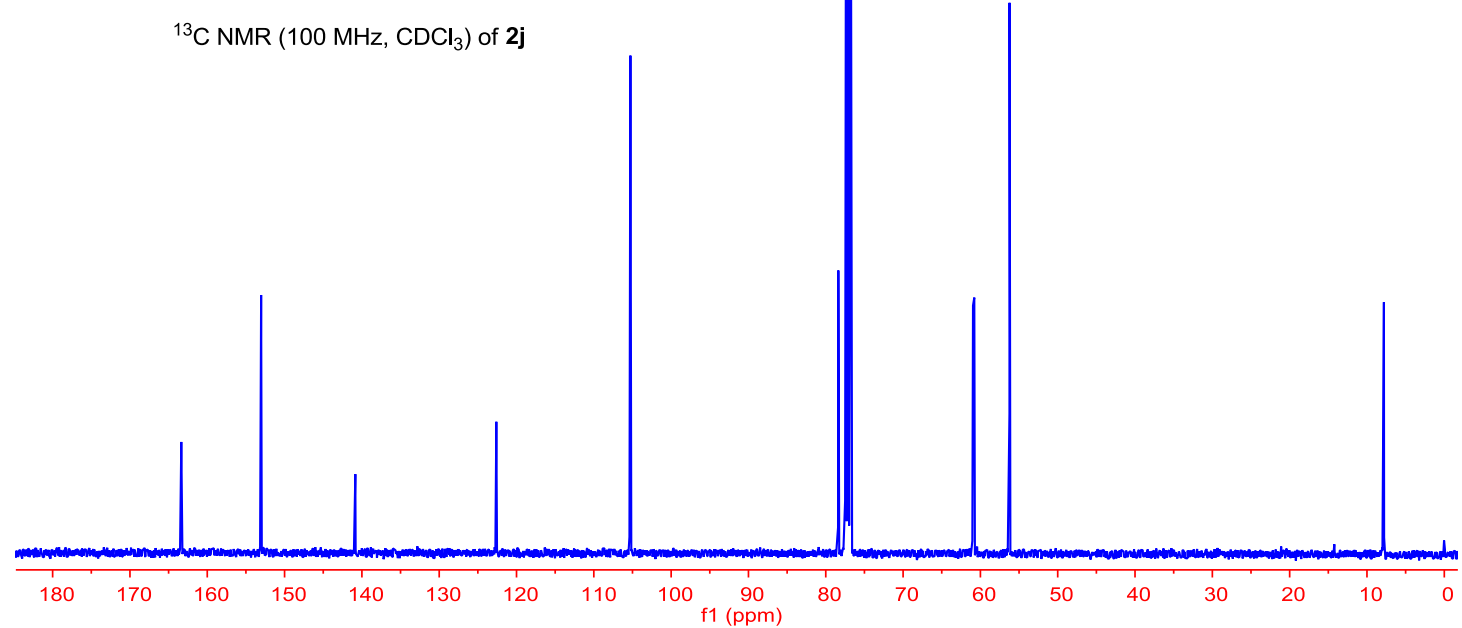




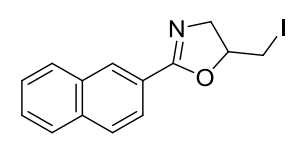

${ }^{1} \mathrm{H}$ NMR $\left(400 \mathrm{MHz}, \mathrm{CDCl}_{3}\right)$ of $\mathbf{2 k}$
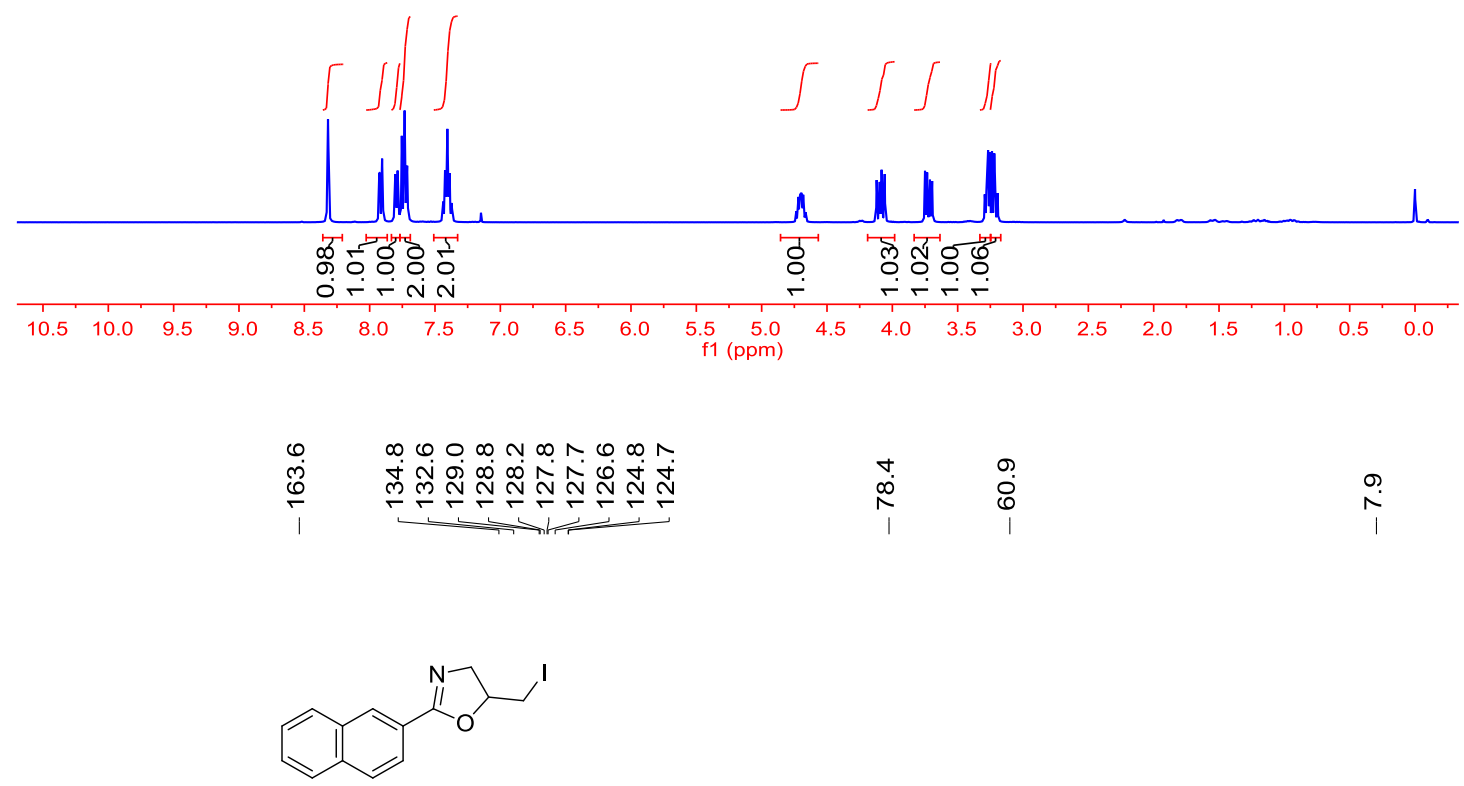

${ }^{13} \mathrm{C} \mathrm{NMR}\left(100 \mathrm{MHz}, \mathrm{CDCl}_{3}\right)$ of $\mathbf{2 k}$

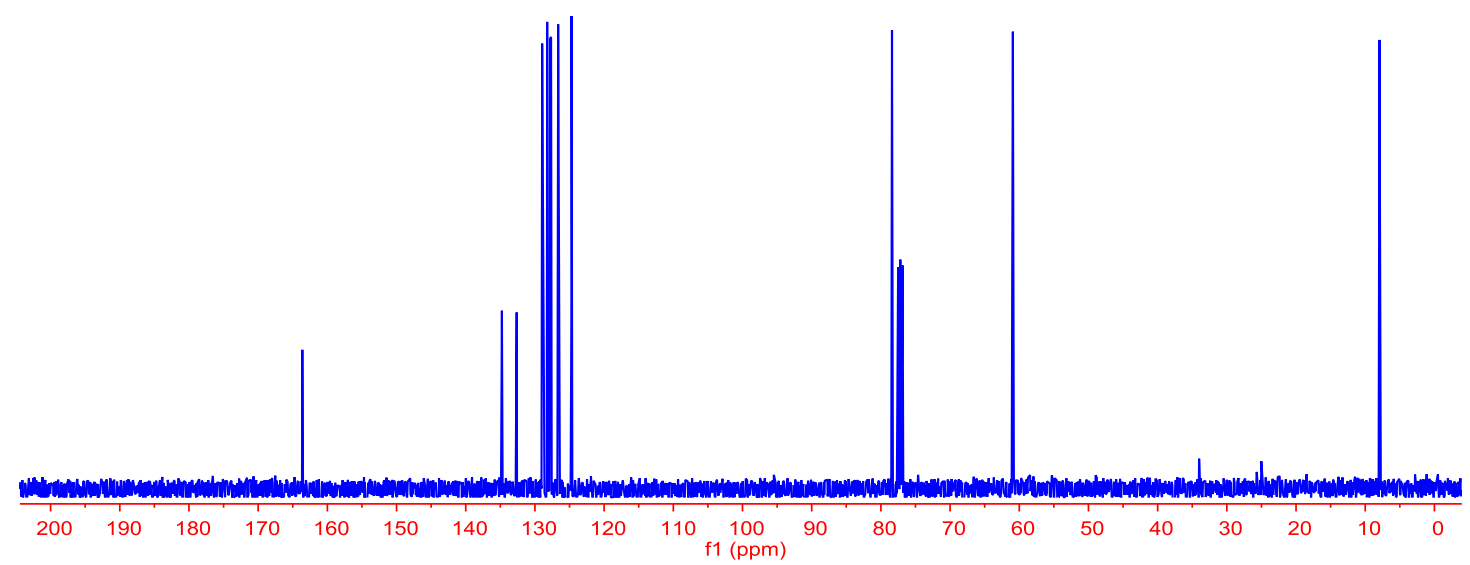




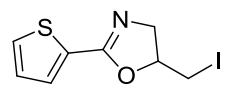

${ }^{1} \mathrm{H} \mathrm{NMR}\left(400 \mathrm{MHz}, \mathrm{CDCl}_{3}\right)$ of 2 I

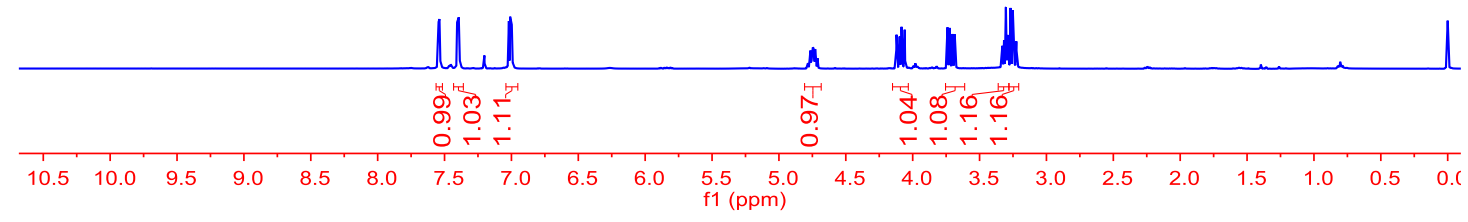

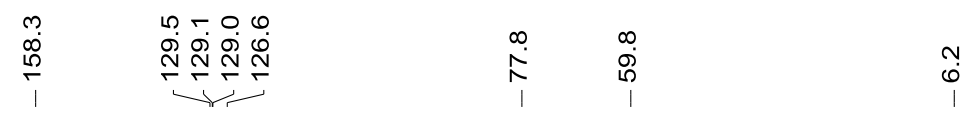

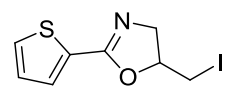

${ }^{13} \mathrm{C} \mathrm{NMR}\left(100 \mathrm{MHz}, \mathrm{CDCl}_{3}\right)$ of 2 I

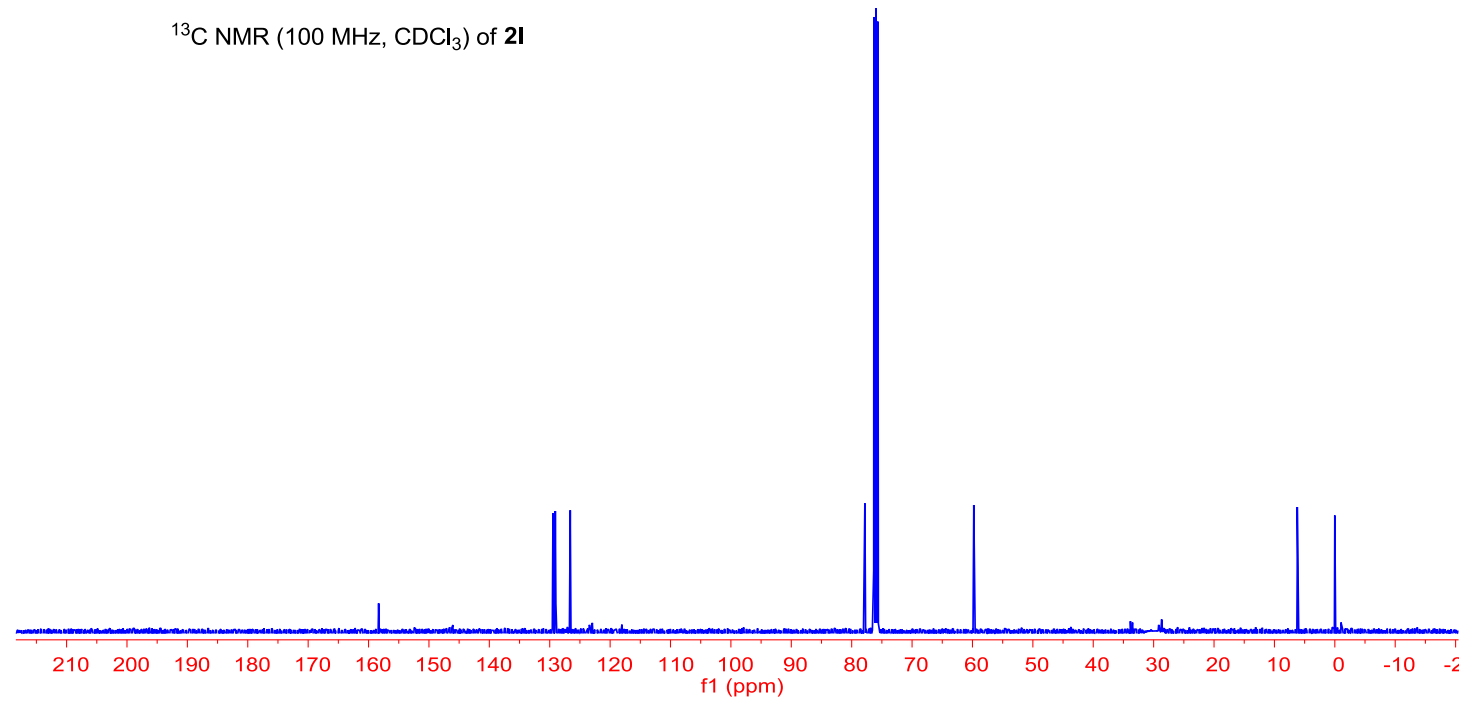




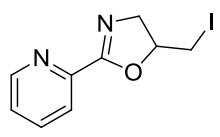

${ }^{1} \mathrm{H} \mathrm{NMR}\left(400 \mathrm{MHz}, \mathrm{CDCl}_{3}\right)$ of $\mathbf{2 m}$

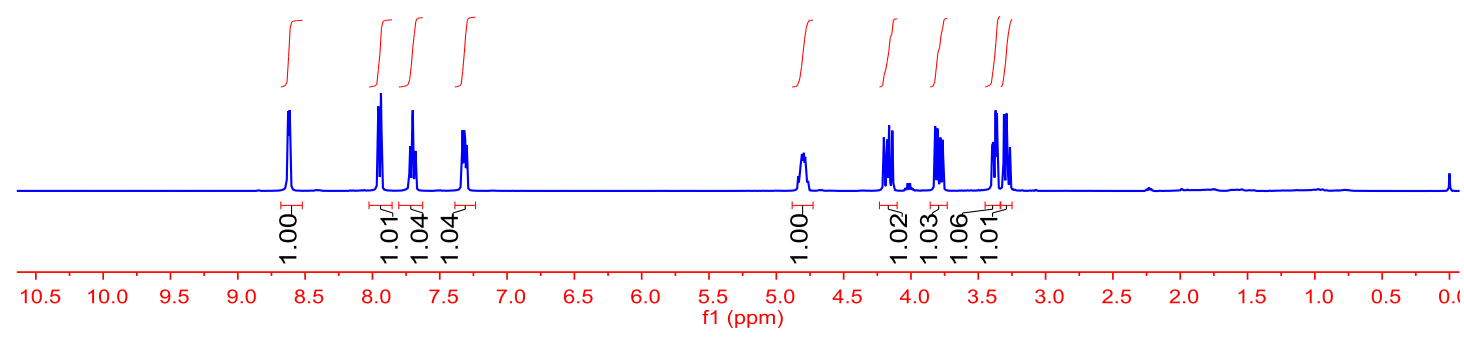

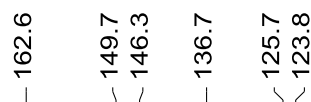

$\begin{array}{ll}\uparrow & 0 \\ i & \dot{0} \\ 1 & 1\end{array}$

$\stackrel{\infty}{i}$

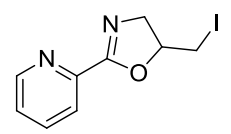

${ }^{13} \mathrm{C}$ NMR $\left(100 \mathrm{MHz}, \mathrm{CDCl}_{3}\right)$ of $\mathbf{2 m}$

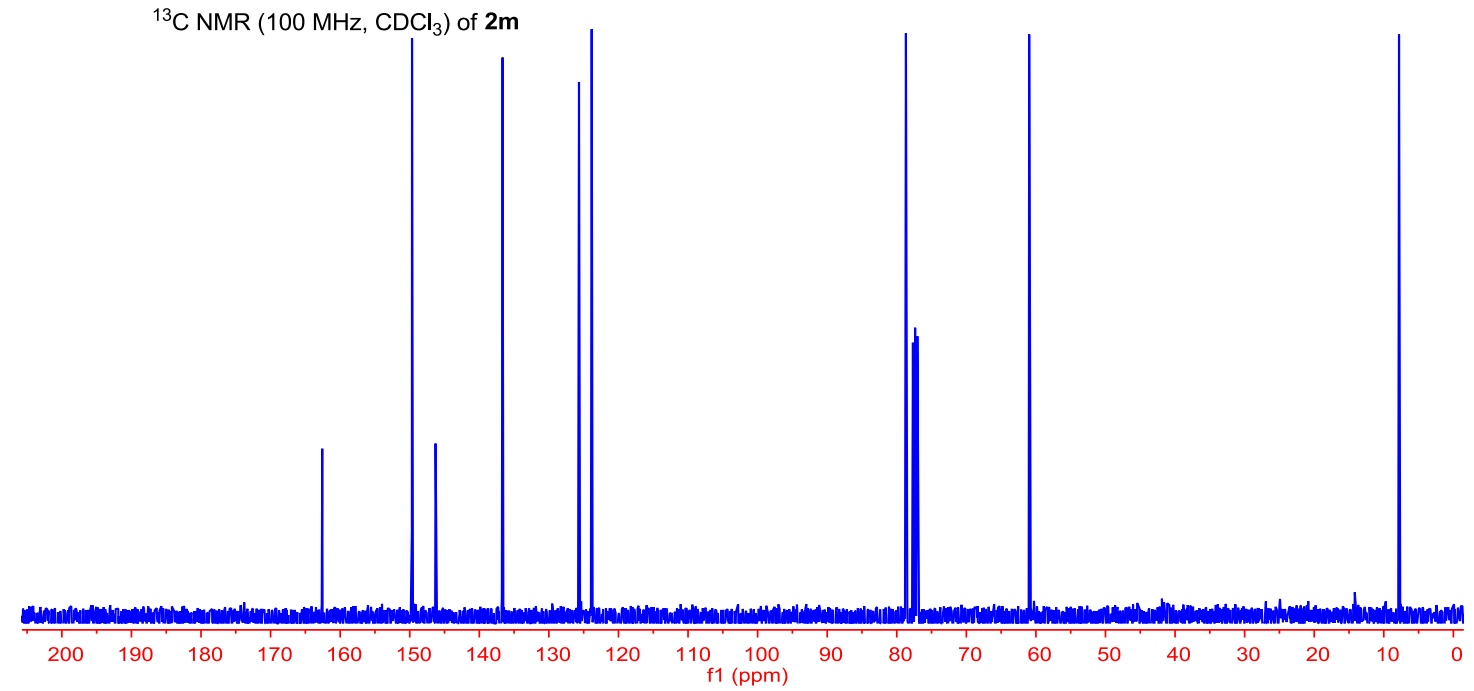




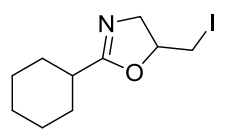

${ }^{1} \mathrm{H} \mathrm{NMR}\left(400 \mathrm{MHz}, \mathrm{CDCl}_{3}\right)$ of $\mathbf{2 n}$
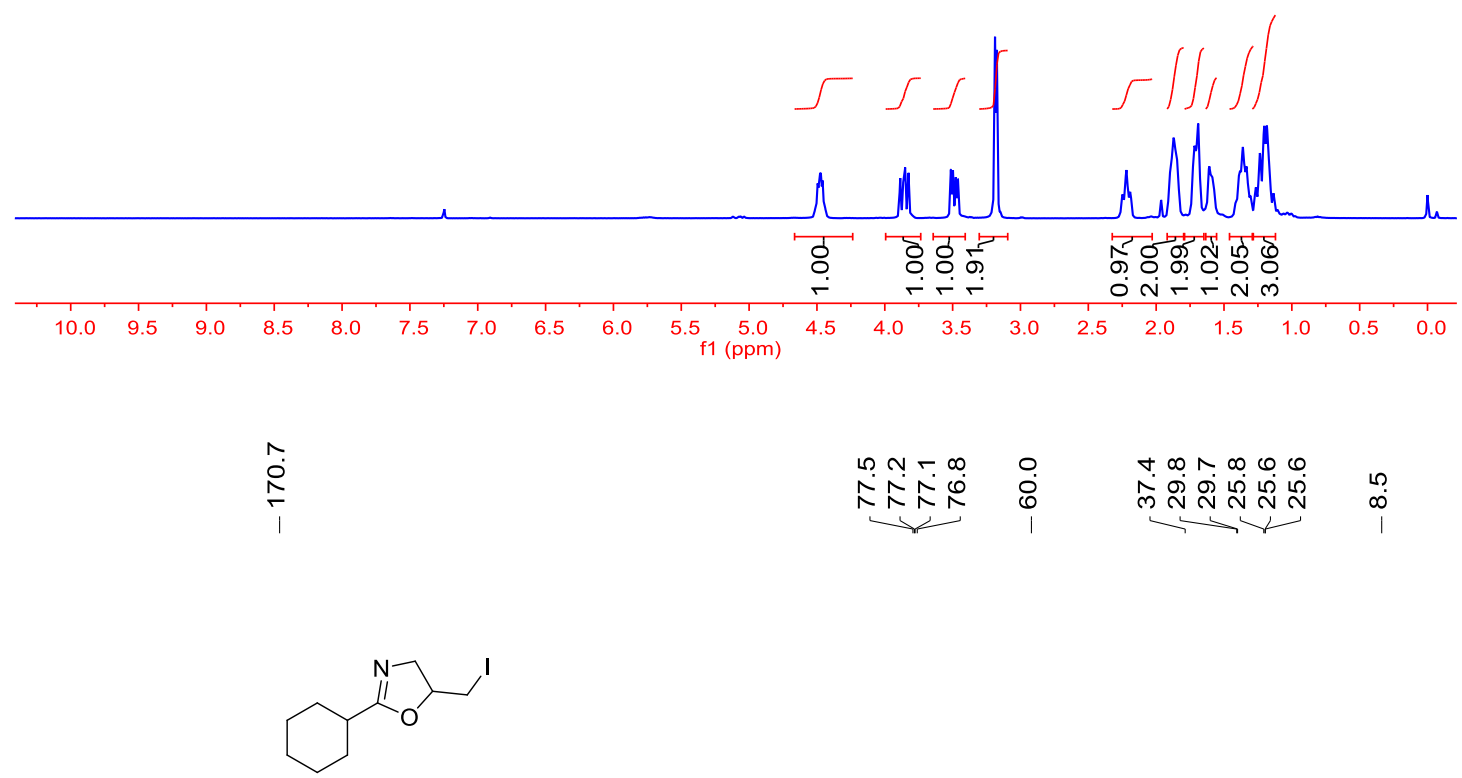

${ }^{13} \mathrm{H} \mathrm{NMR}\left(100 \mathrm{MHz}, \mathrm{CDCl}_{3}\right)$ of $\mathbf{2 n}$

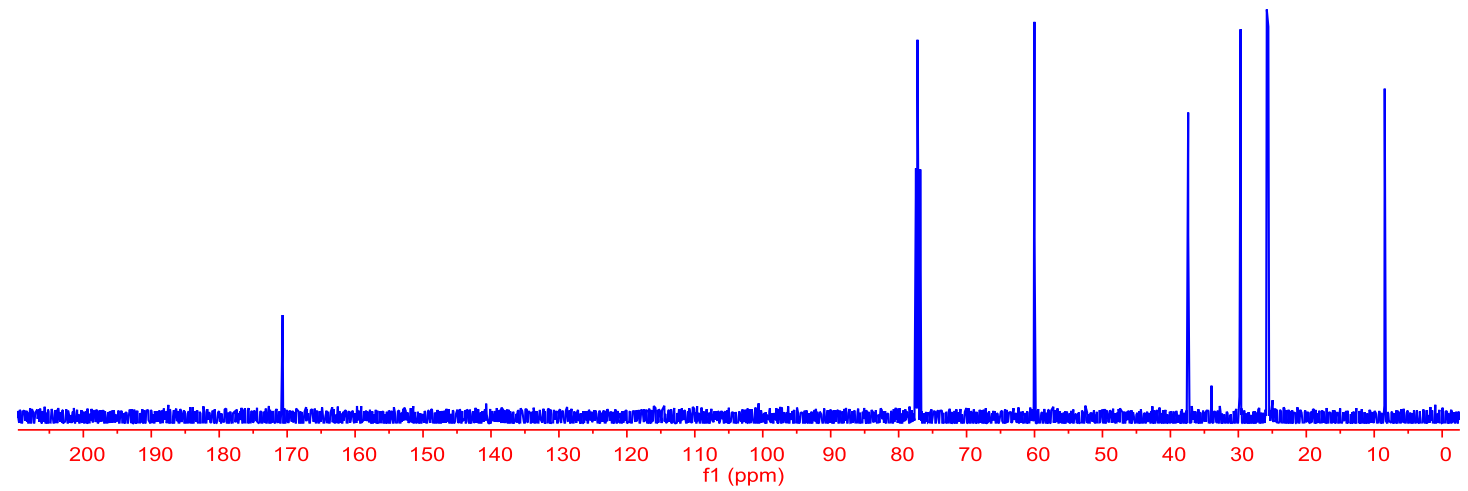




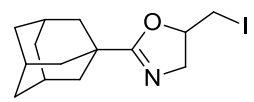

${ }^{1} \mathrm{H}$ NMR $\left(400 \mathrm{MHz}, \mathrm{CDCl}_{3}\right)$ of 20

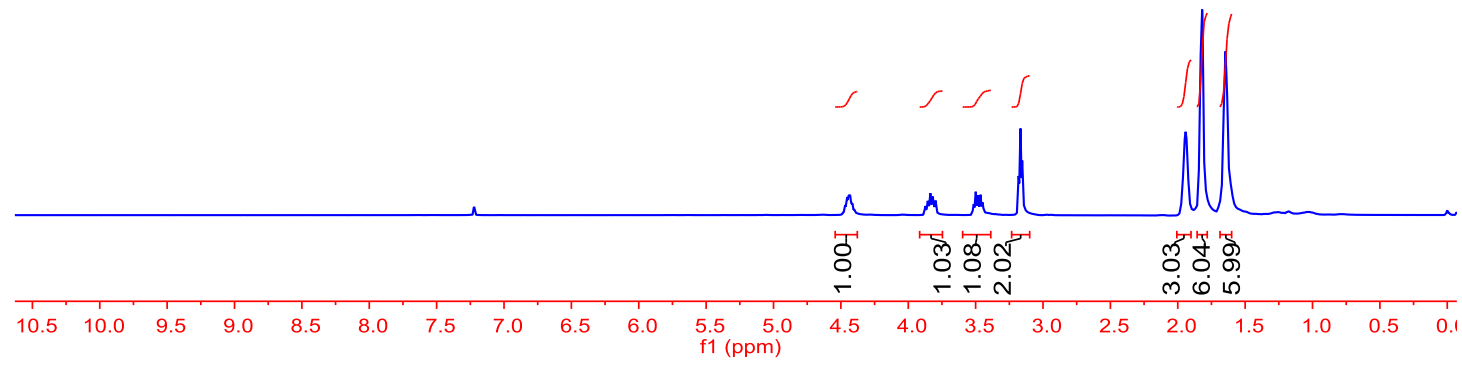

$\stackrel{N}{N}$

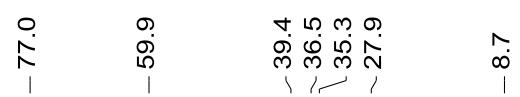

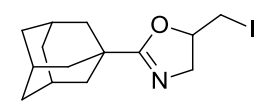

${ }^{13} \mathrm{C} \mathrm{NMR}\left(100 \mathrm{MHz}, \mathrm{CDCl}_{3}\right)$ of 20

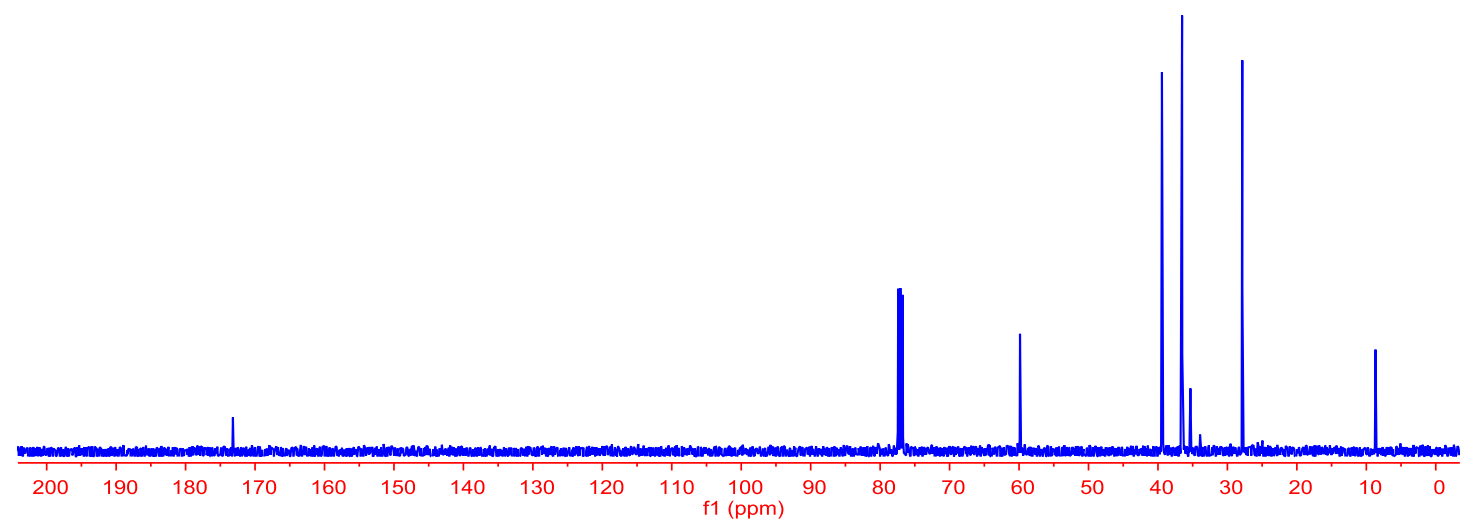




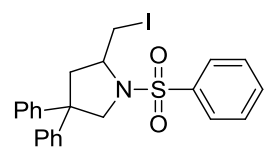

${ }^{1} \mathrm{H} \mathrm{NMR}\left(400 \mathrm{MHz}, \mathrm{CDCl}_{3}\right)$ of $\mathbf{4 a}$
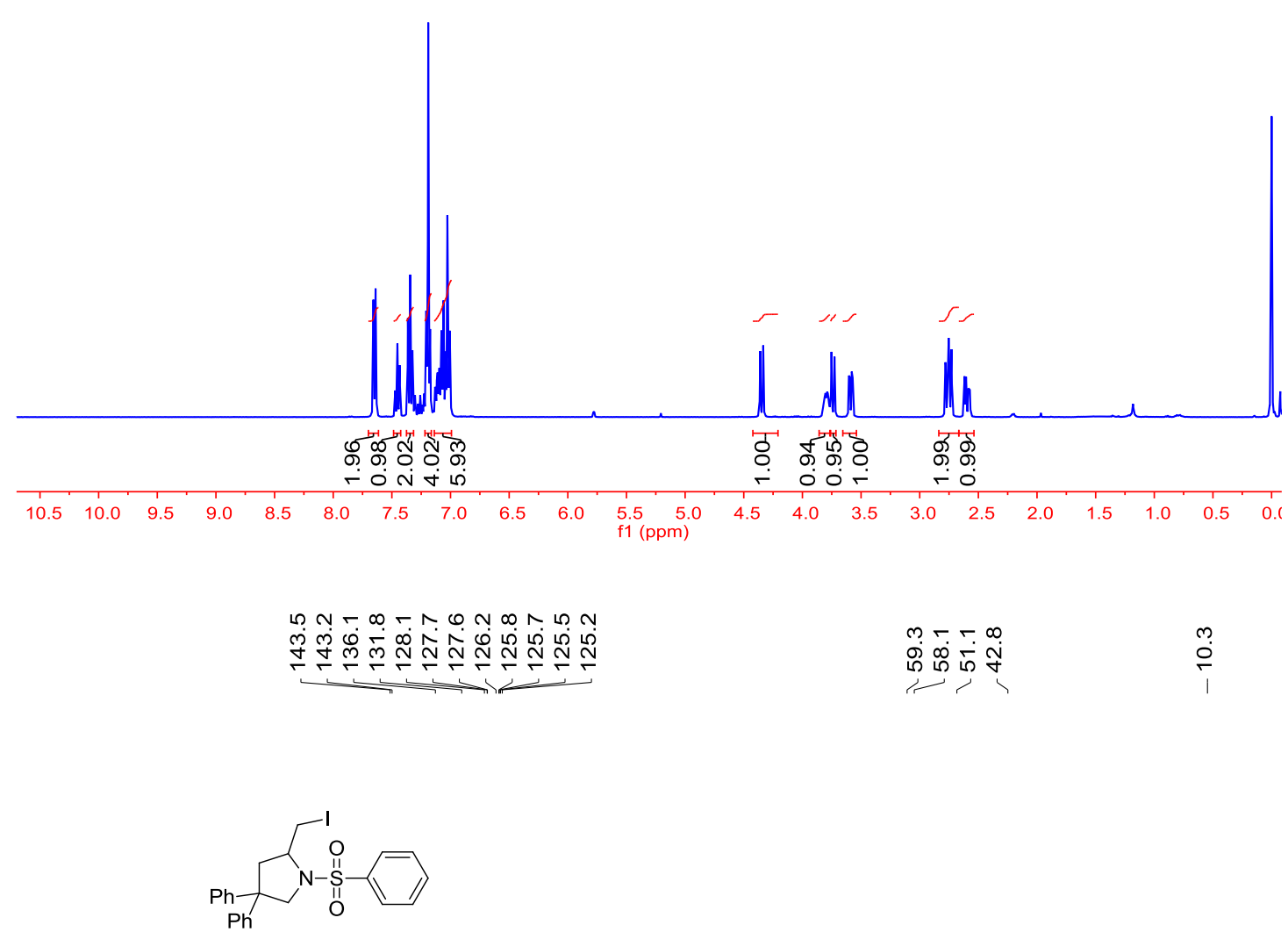

${ }^{13} \mathrm{C} \mathrm{NMR}\left(100 \mathrm{MHz}, \mathrm{CDCl}_{3}\right)$ of $4 \mathrm{a}$

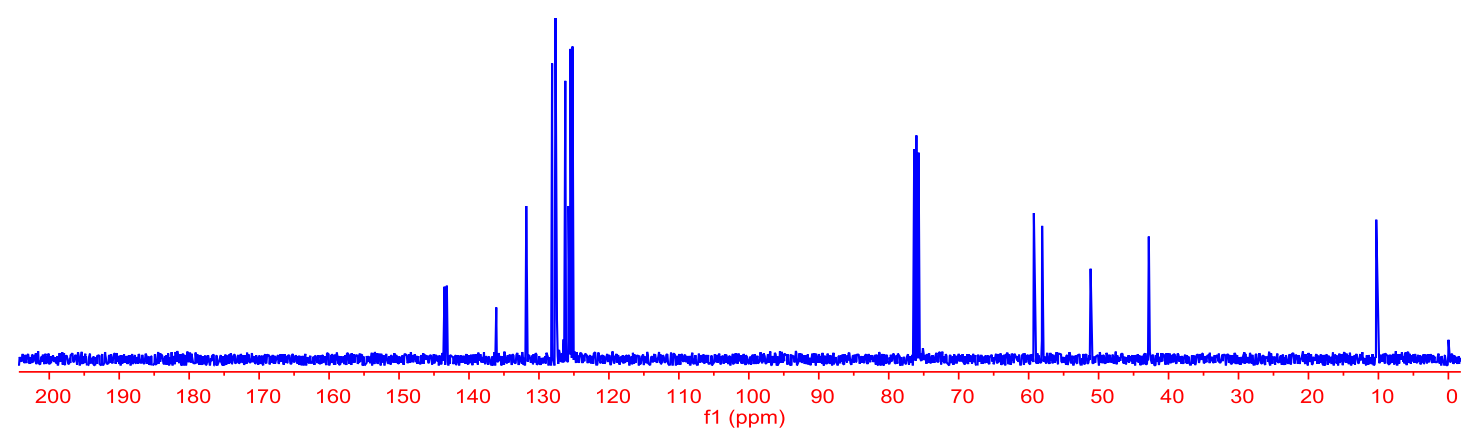




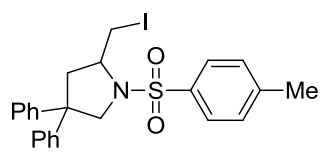

${ }^{1} \mathrm{H} \mathrm{NMR}\left(400 \mathrm{MHz}, \mathrm{CDCl}_{3}\right)$ of $\mathbf{4 b}$
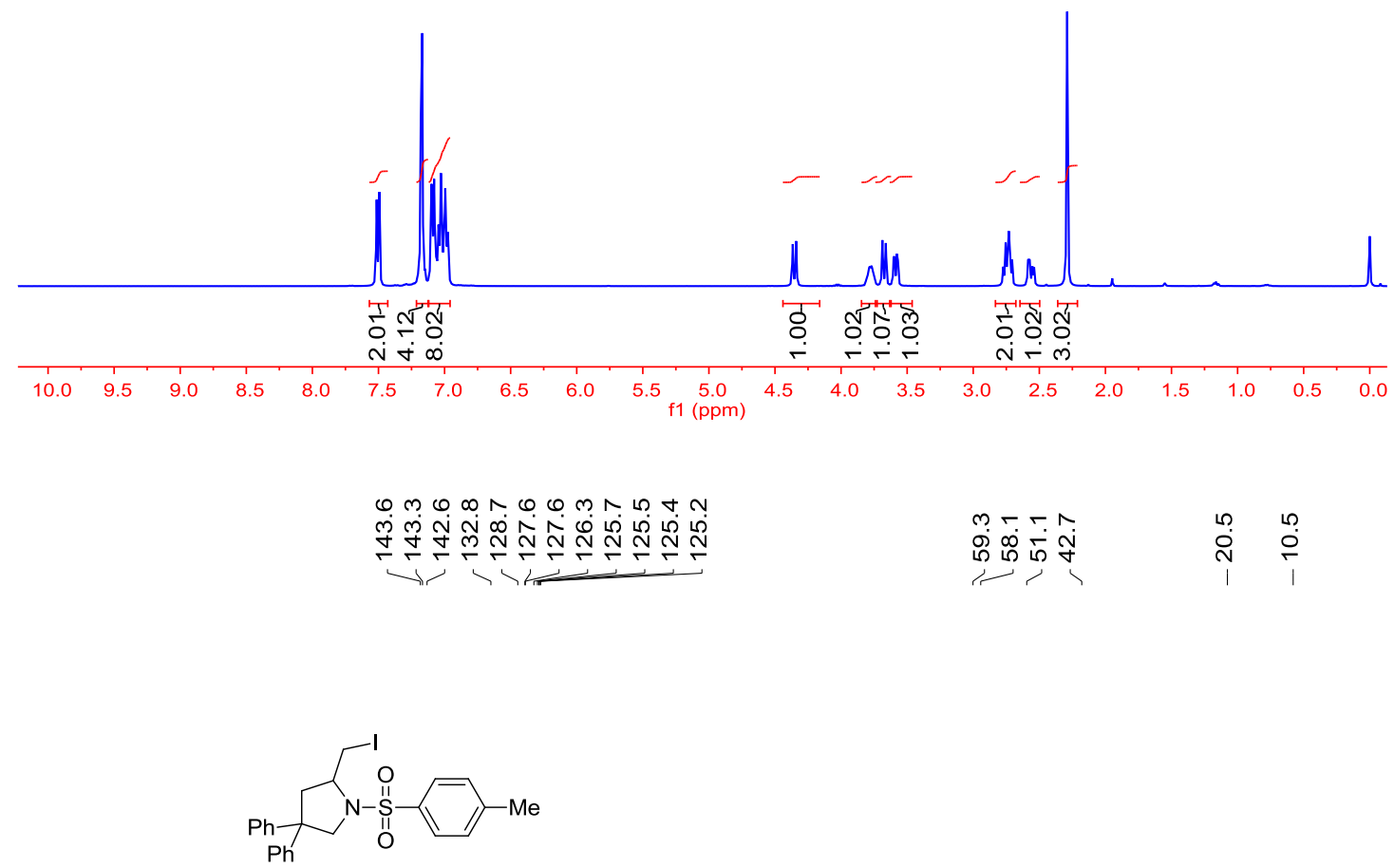

${ }^{13} \mathrm{H}$ NMR $\left(100 \mathrm{MHz}, \mathrm{CDCl}_{3}\right)$ of $\mathbf{4 b}$

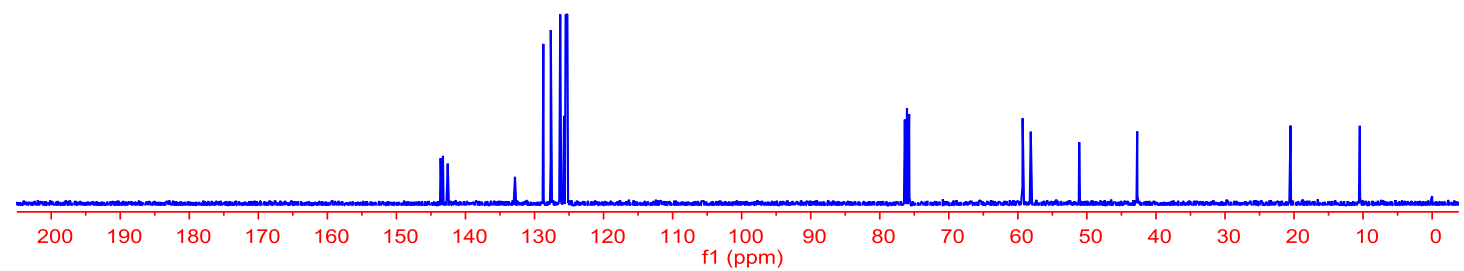




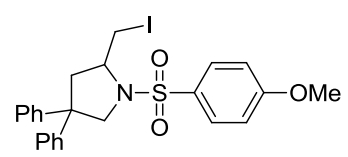

${ }^{1} \mathrm{H} \mathrm{NMR}\left(400 \mathrm{MHz}, \mathrm{CDCl}_{3}\right)$ of $4 \mathrm{c}$

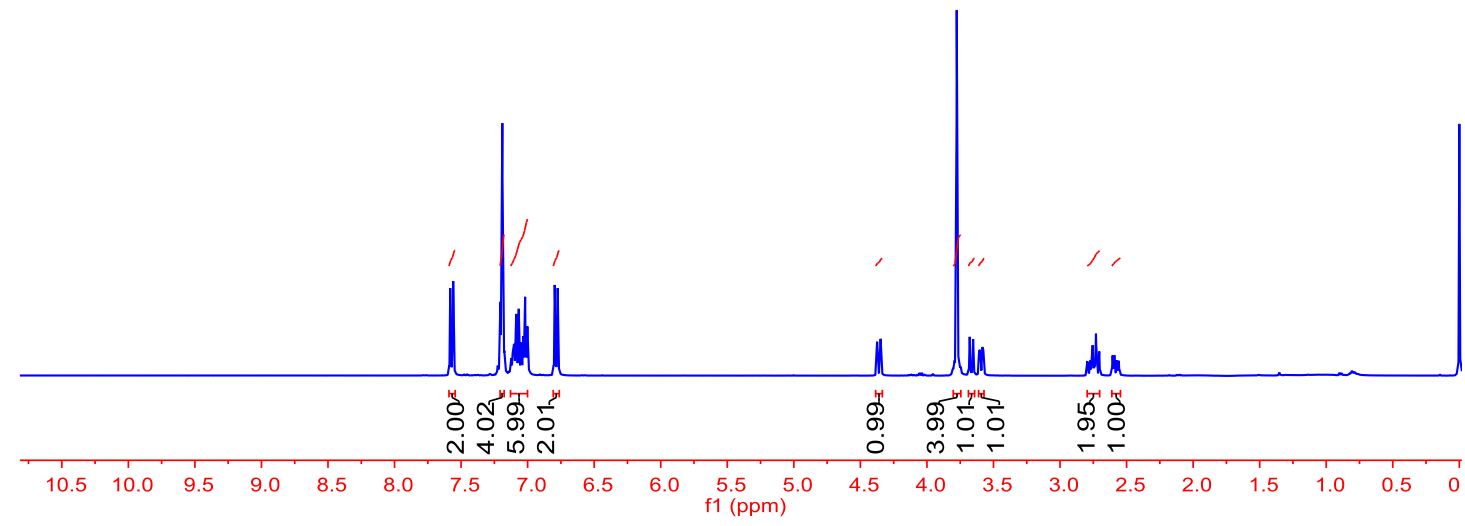

Oํ.

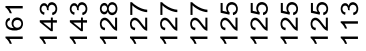

$\forall$ ก

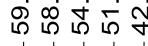

$\stackrel{1}{\stackrel{2}{0}}$

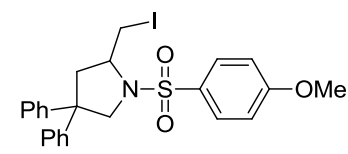

${ }^{13} \mathrm{C}$ NMR $\left(100 \mathrm{MHz}, \mathrm{CDCl}_{3}\right)$ of $4 \mathrm{c}$

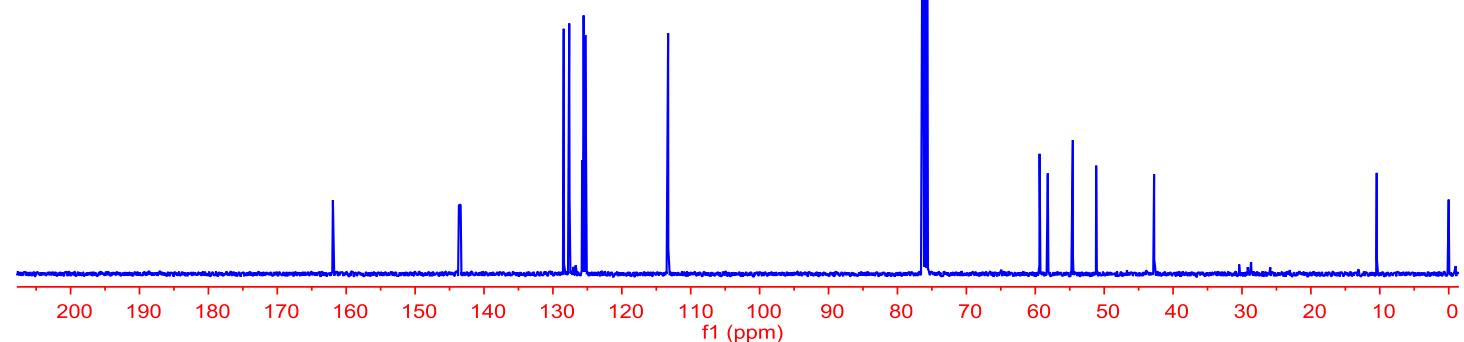




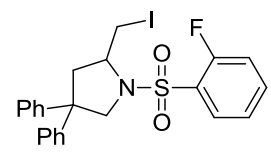

${ }^{1} \mathrm{H} \mathrm{NMR}\left(400 \mathrm{MHz}, \mathrm{CDCl}_{3}\right)$ of $\mathbf{4 d}$
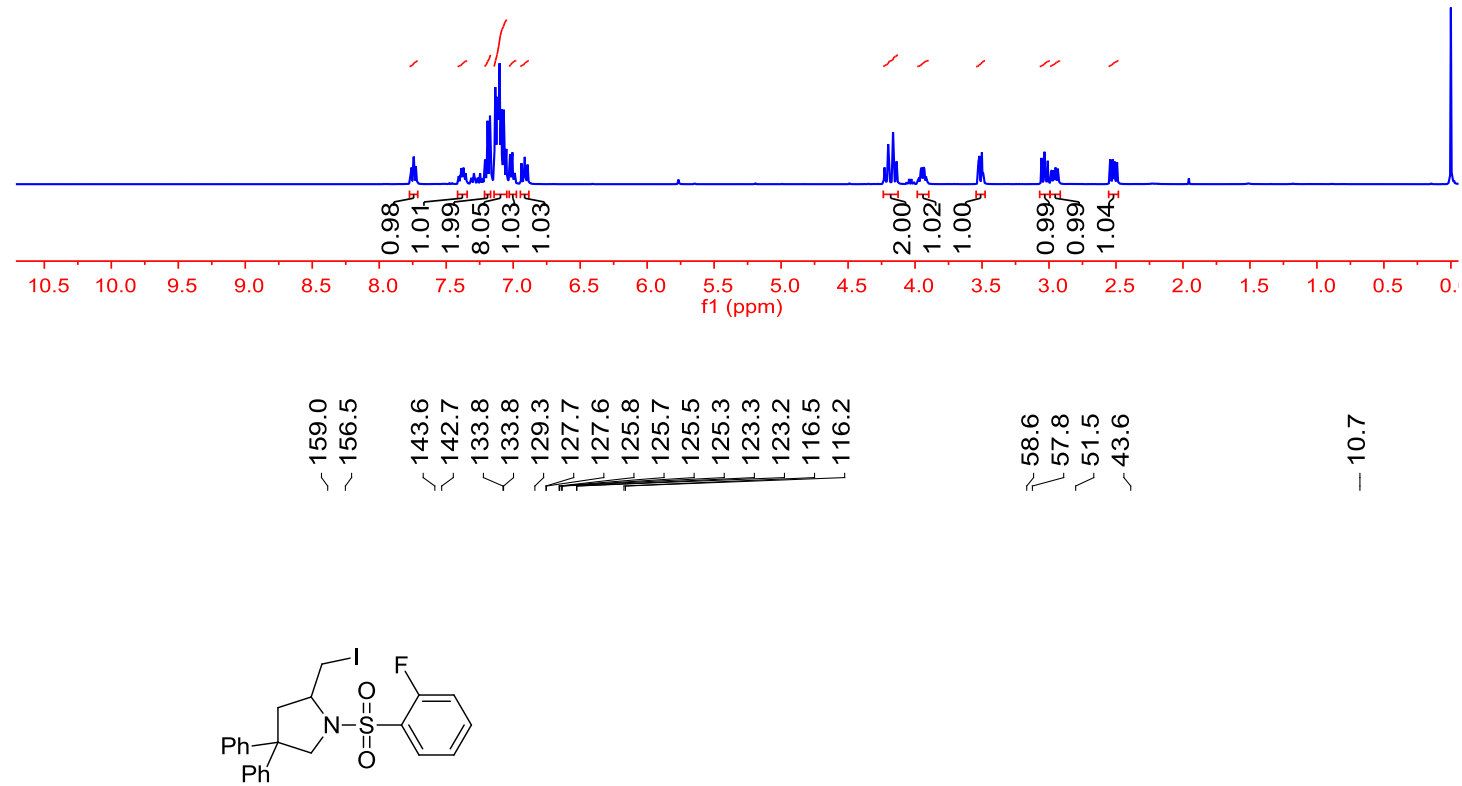

${ }^{13} \mathrm{C} \mathrm{NMR}\left(100 \mathrm{MHz}, \mathrm{CDCl}_{3}\right)$ of $\mathbf{4 d}$

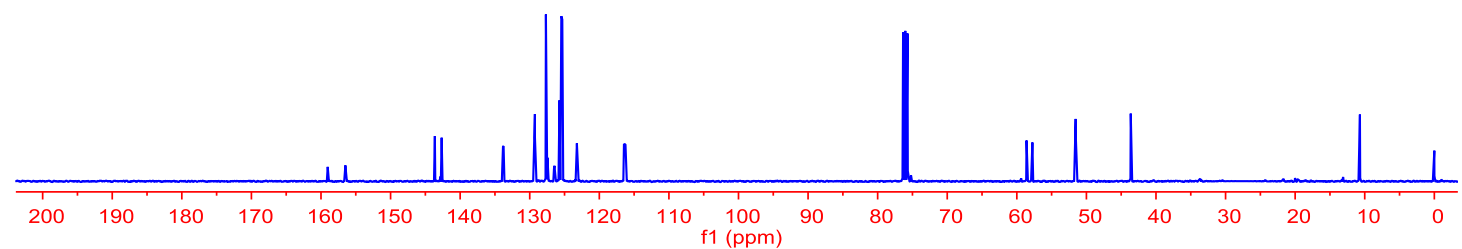




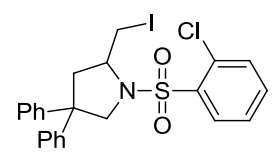

${ }^{1} \mathrm{H}$ NMR $\left(400 \mathrm{MHz}, \mathrm{CDCl}_{3}\right)$ of $\mathbf{4 e}$
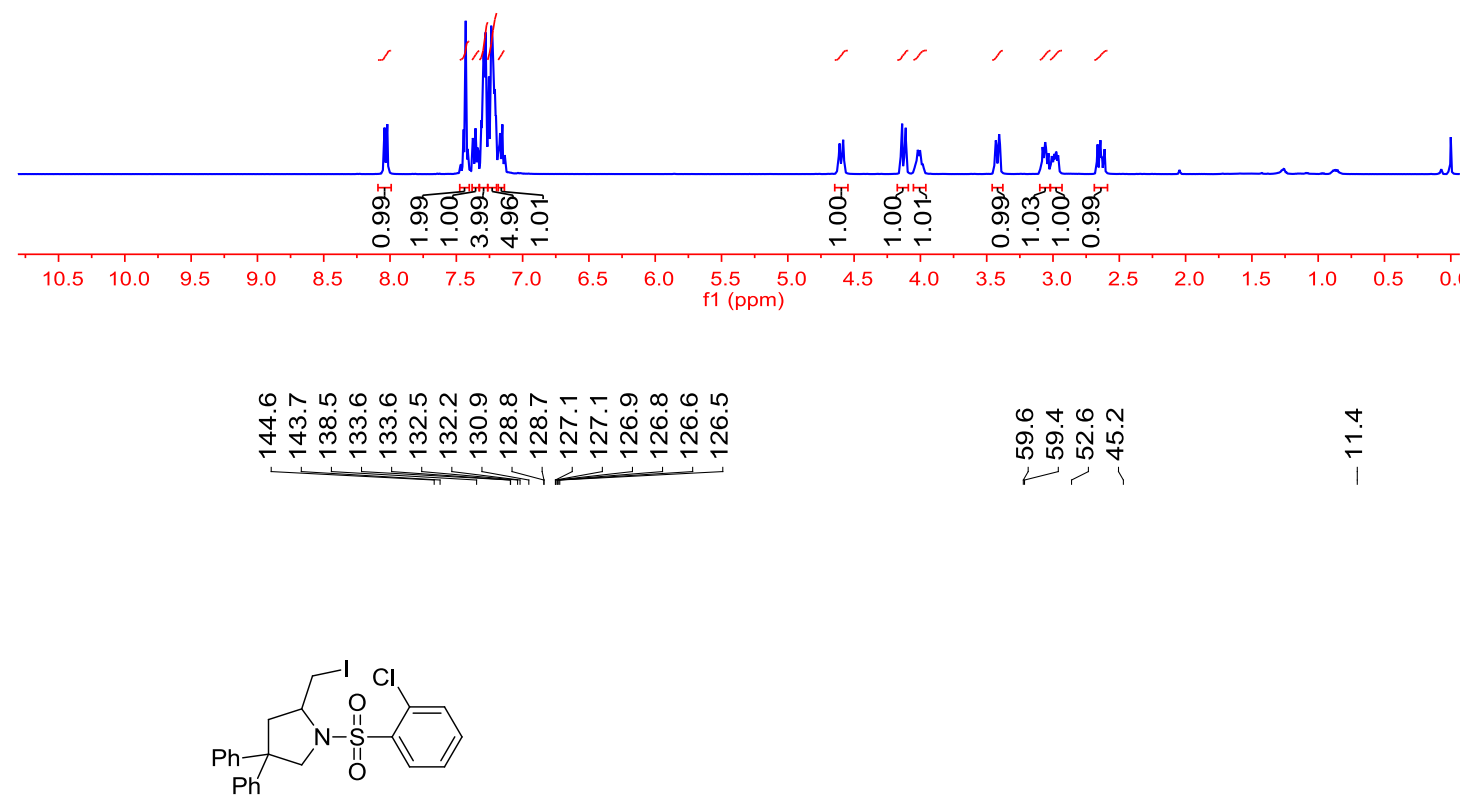

${ }^{13} \mathrm{C}$ NMR $\left(100 \mathrm{MHz}, \mathrm{CDCl}_{3}\right)$ of $4 \mathbf{e}$

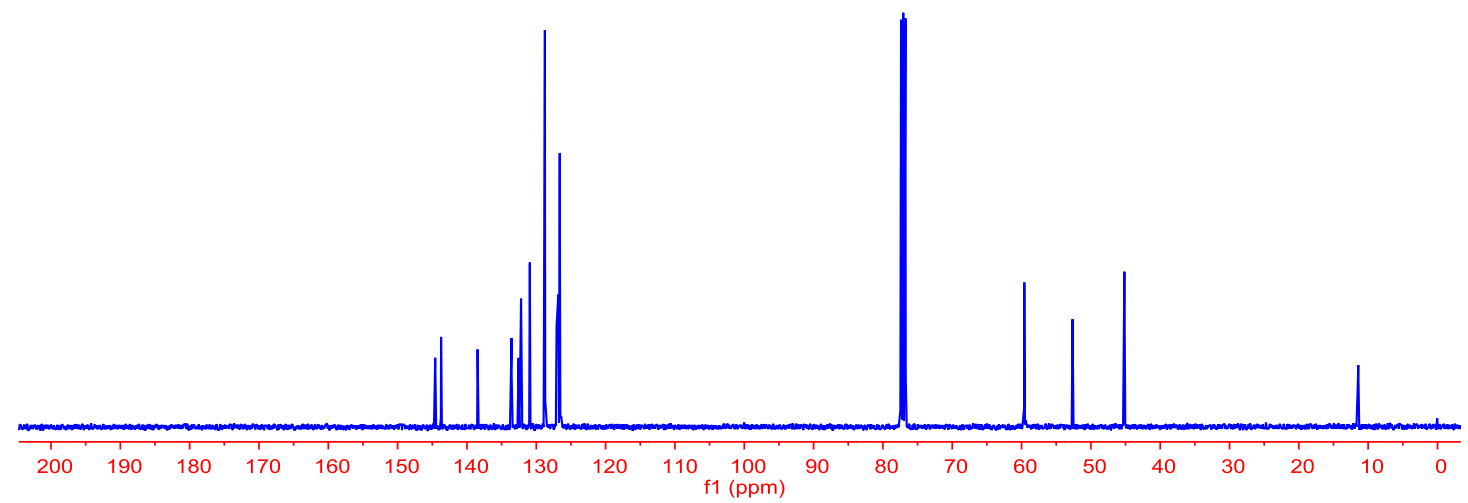




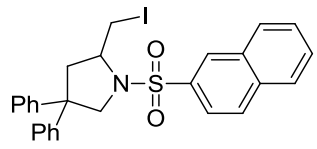

${ }^{1} \mathrm{H} \mathrm{NMR}\left(400 \mathrm{MHz}, \mathrm{CDCl}_{3}\right)$ of $\mathbf{4 f}$
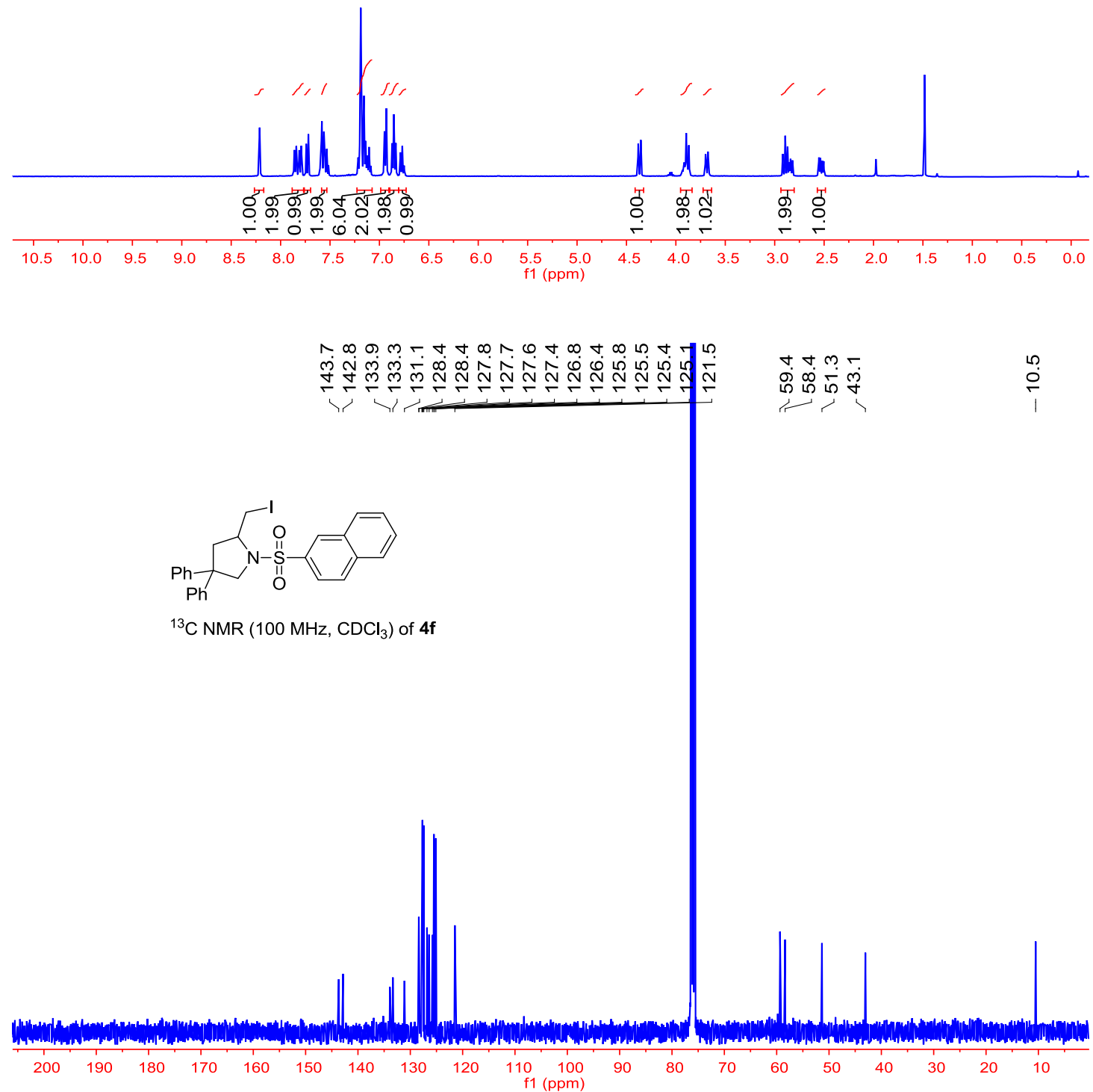


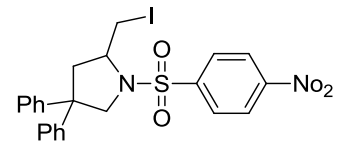

${ }^{1} \mathrm{H}$ NMR $\left(400 \mathrm{MHz}, \mathrm{CDCl}_{3}\right)$ of $\mathbf{4 g}$

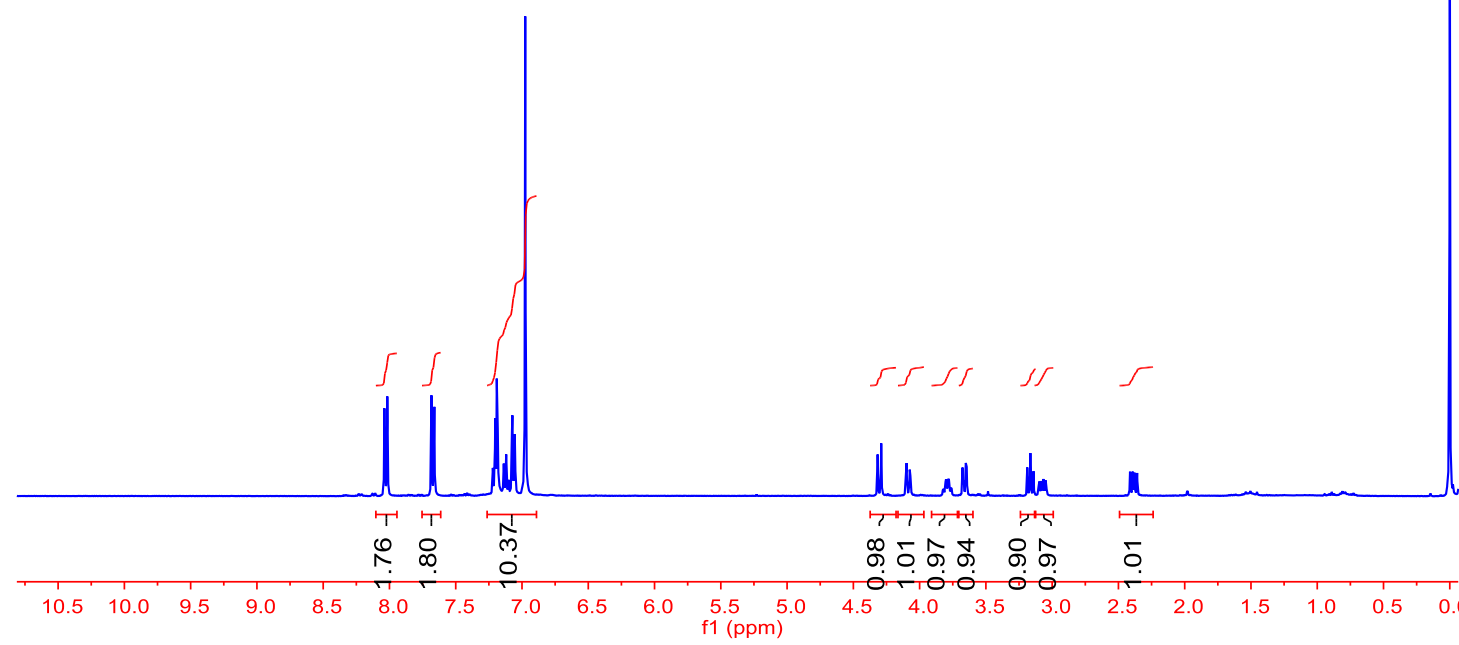

$\infty+\Delta m \infty$ t.

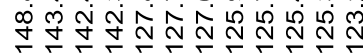

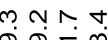

迥吕的寽

$\stackrel{\circ}{\circ}$

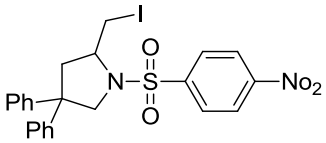

${ }^{13} \mathrm{C} \mathrm{NMR}\left(100 \mathrm{MHz}, \mathrm{CDCl}_{3}\right)$ of $\mathbf{4 g}$

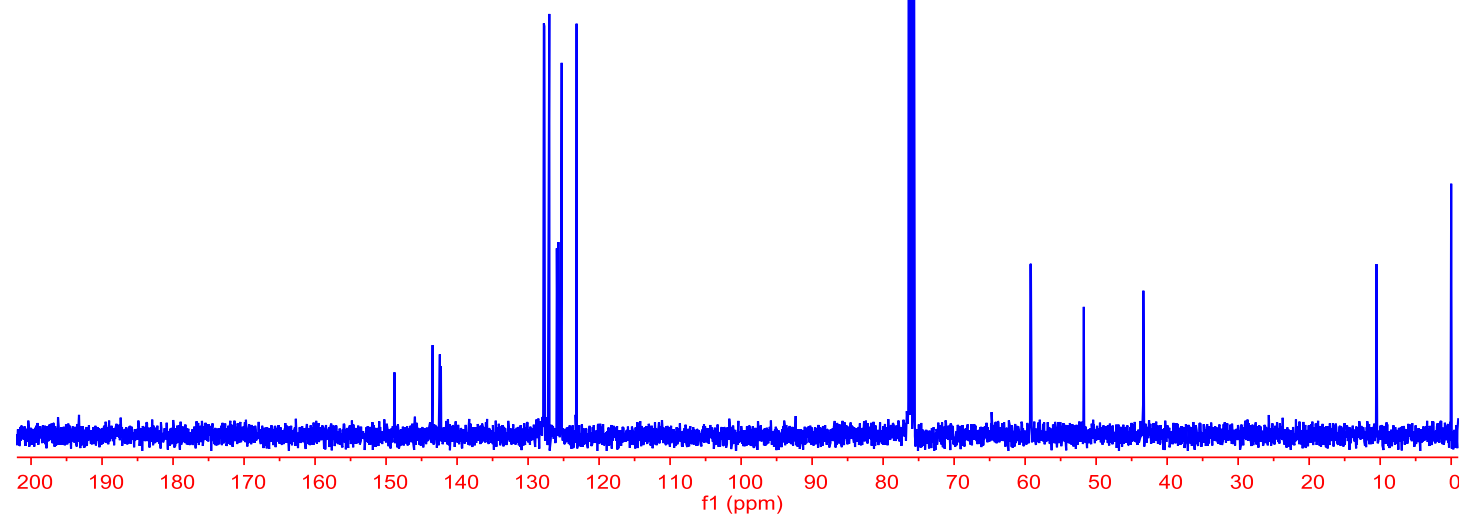




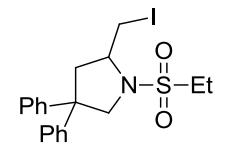

${ }^{1} \mathrm{H} \mathrm{NMR}\left(400 \mathrm{MHz}, \mathrm{CDCl}_{3}\right)$ of $4 \mathrm{~h}$
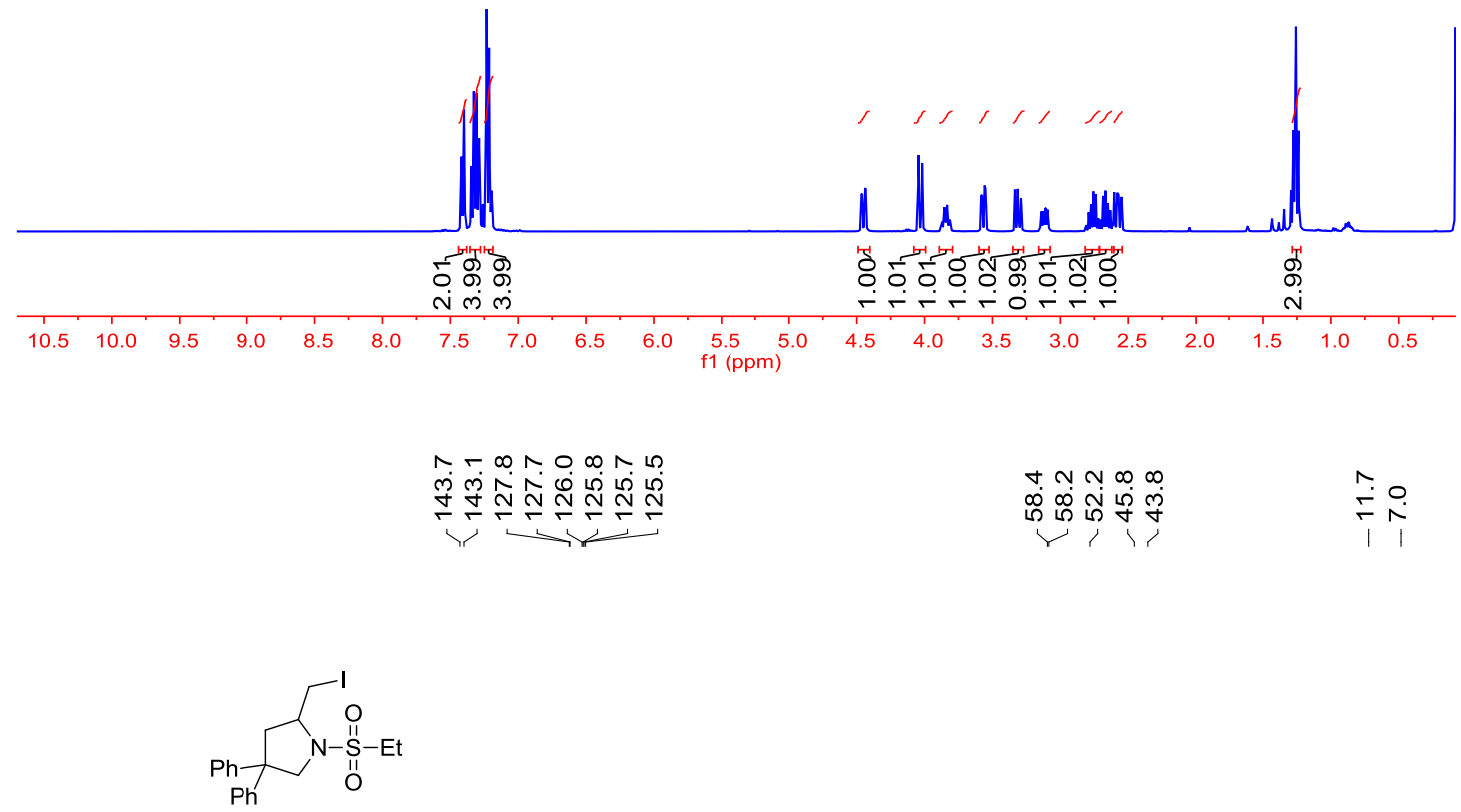

${ }^{13} \mathrm{C} \mathrm{NMR}\left(100 \mathrm{MHz}, \mathrm{CDCl}_{3}\right)$ of $4 \mathrm{~h}$

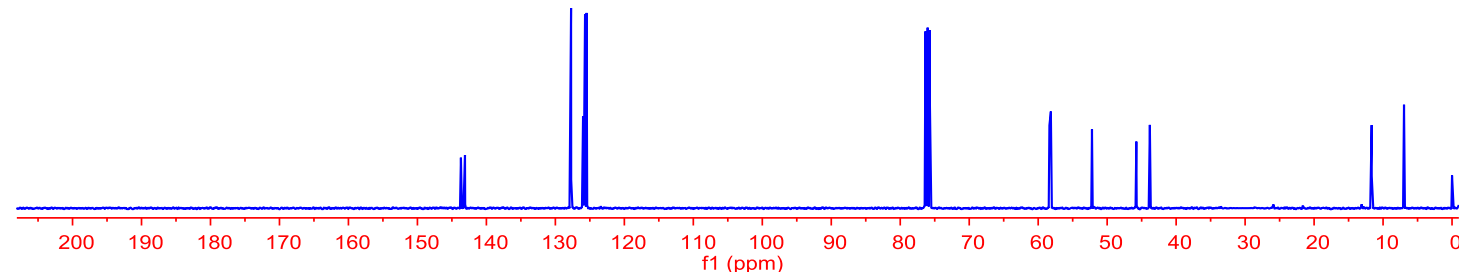




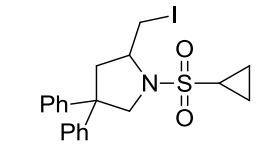

${ }^{1} \mathrm{H} \mathrm{NMR}\left(400 \mathrm{MHz}, \mathrm{CDCl}_{3}\right)$ of $4 \mathrm{i}$

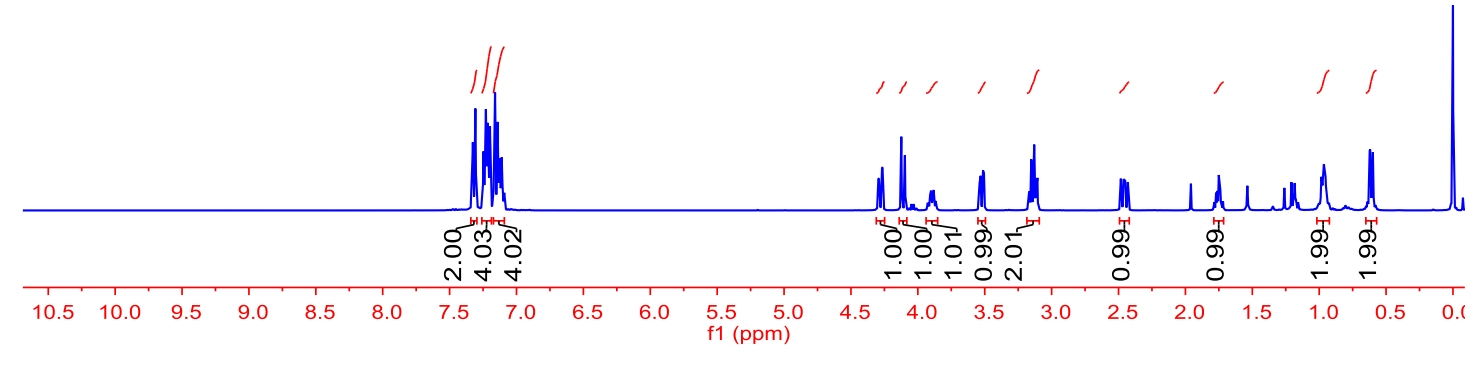

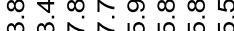

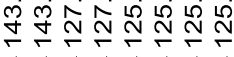

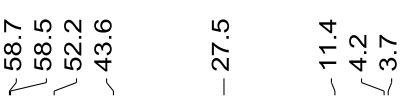

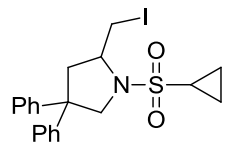

${ }^{13} \mathrm{C} \mathrm{NMR}\left(100 \mathrm{MHz}, \mathrm{CDCl}_{3}\right)$ of $4 \mathrm{i}$

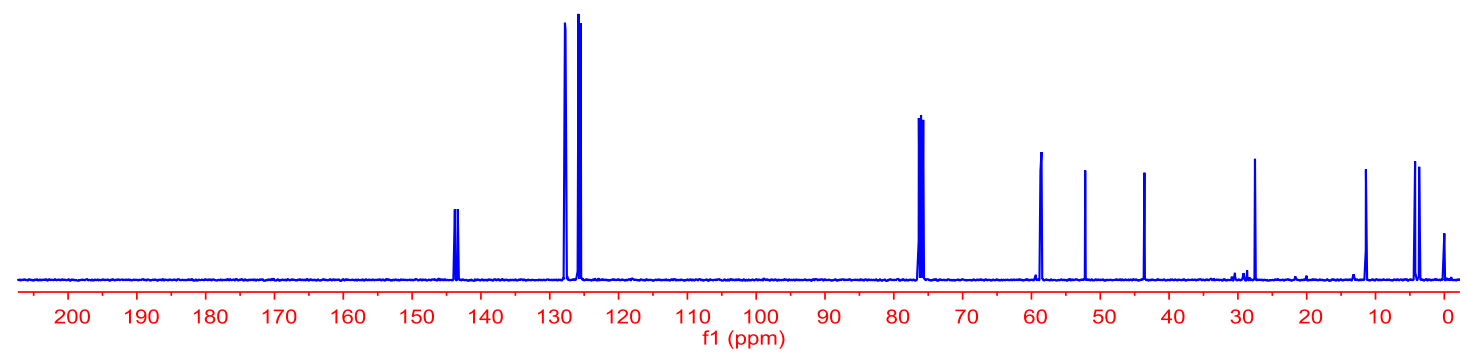




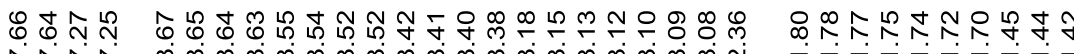

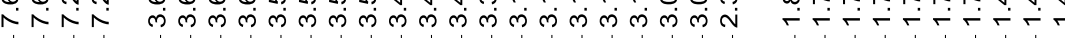

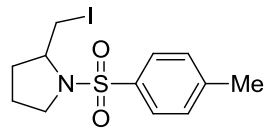

${ }^{1} \mathrm{H} \mathrm{NMR}\left(400 \mathrm{MHz}, \mathrm{CDCl}_{3}\right)$ of $4 \mathrm{j}$

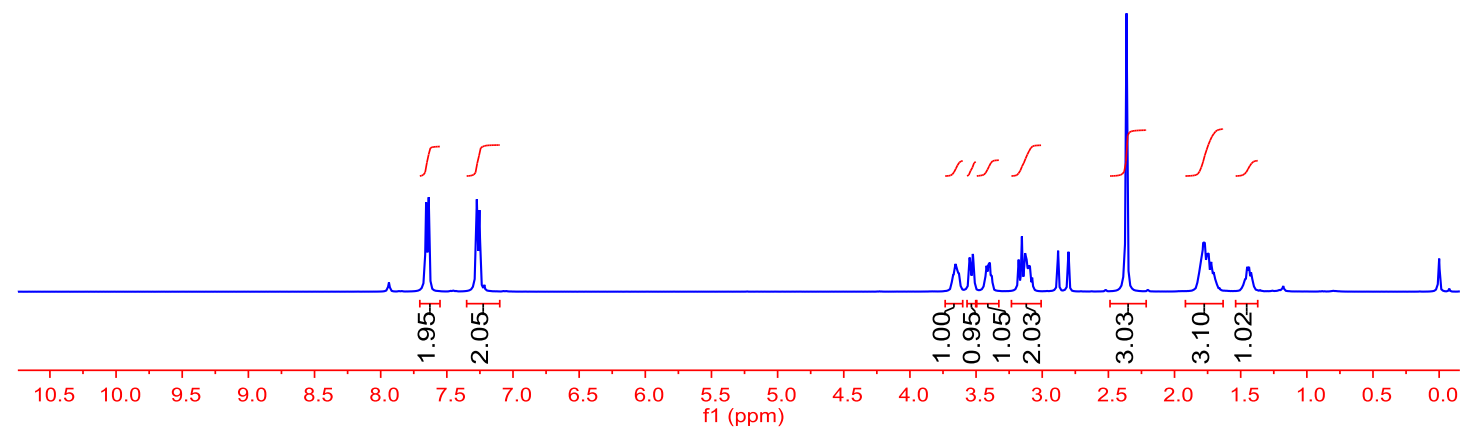

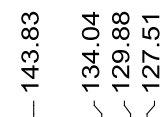

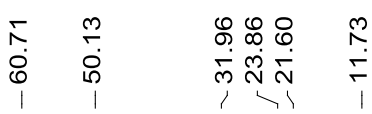

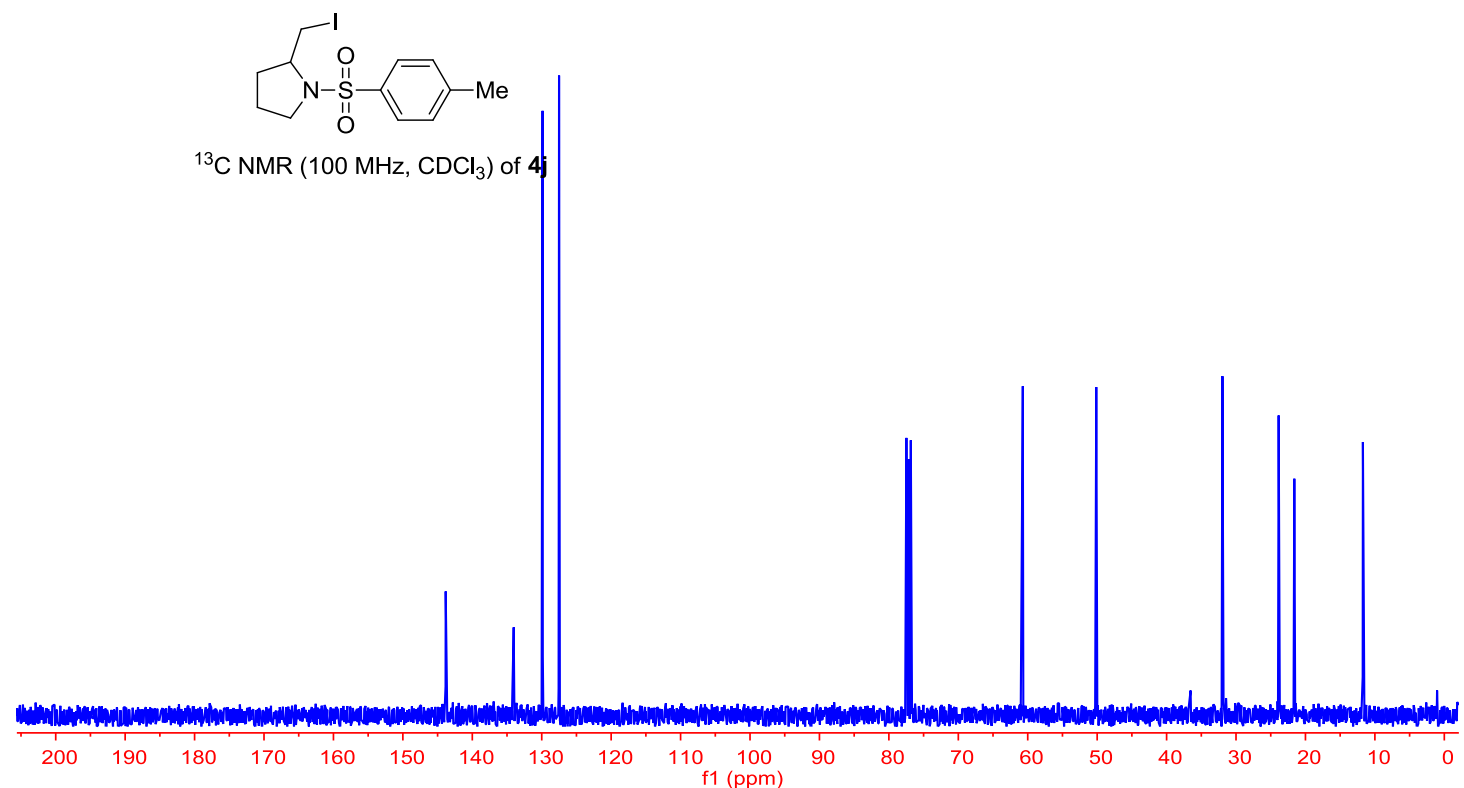




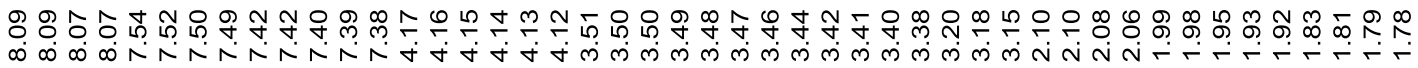

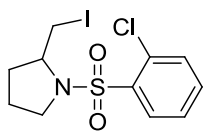

${ }^{1} \mathrm{H} \mathrm{NMR}\left(400 \mathrm{MHz}, \mathrm{CDCl}_{3}\right)$ of $\mathbf{4 k}$

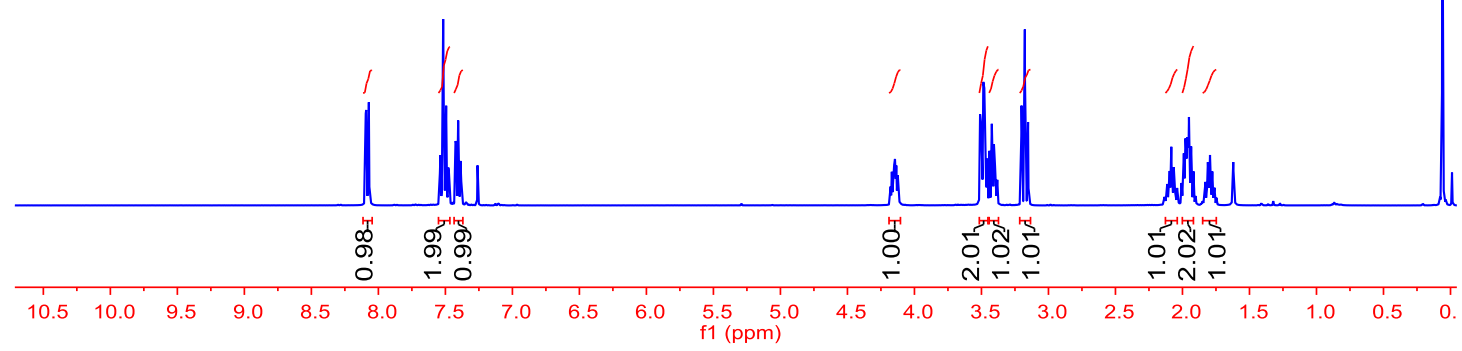

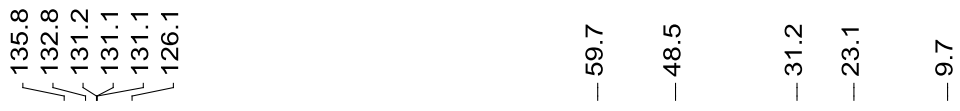

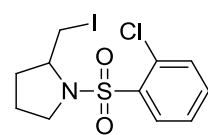

${ }^{13} \mathrm{C} \mathrm{NMR}\left(100 \mathrm{MHz}, \mathrm{CDCl}_{3}\right)$ of $\mathbf{4 k}$

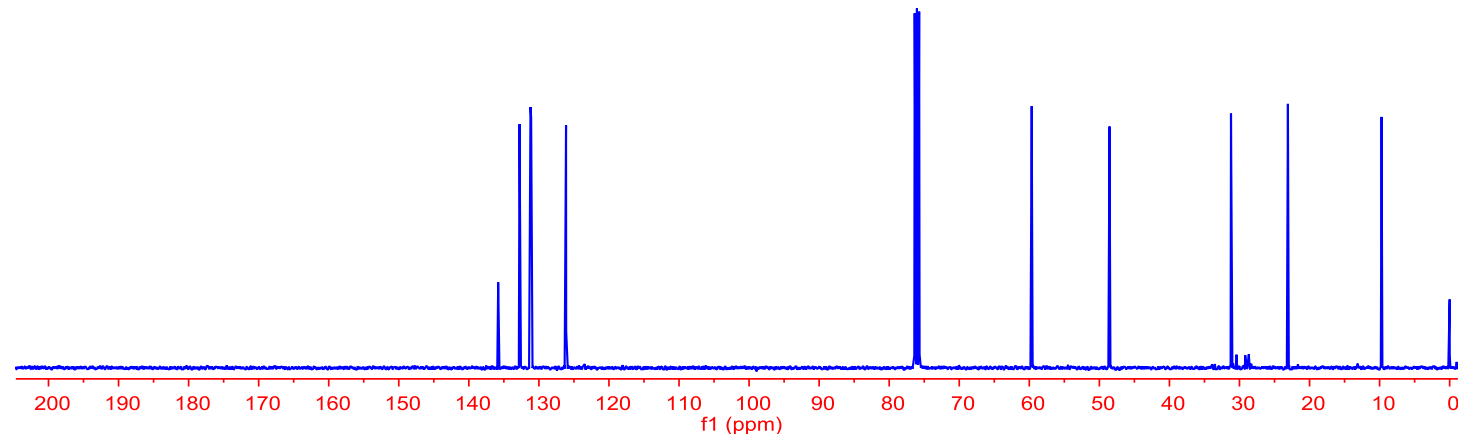




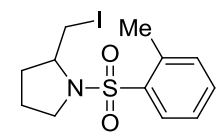

${ }^{1} \mathrm{H}$ NMR $\left(400 \mathrm{MHz}, \mathrm{CDCl}_{3}\right)$ of $4 \mathbf{I}$

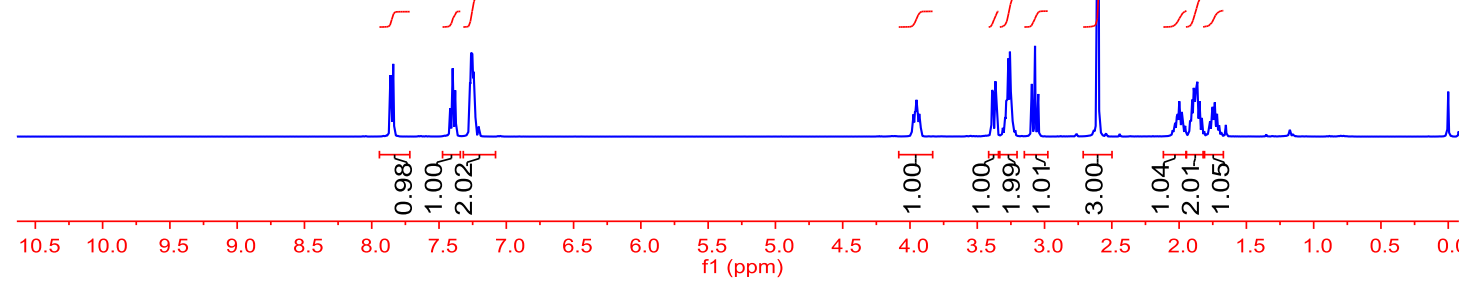

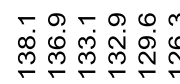

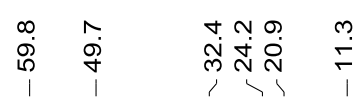

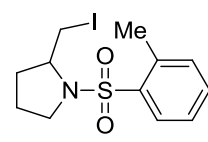

${ }^{13} \mathrm{C} \mathrm{NMR}\left(100 \mathrm{MHz}, \mathrm{CDCl}_{3}\right)$ of $4 \mathrm{I}$

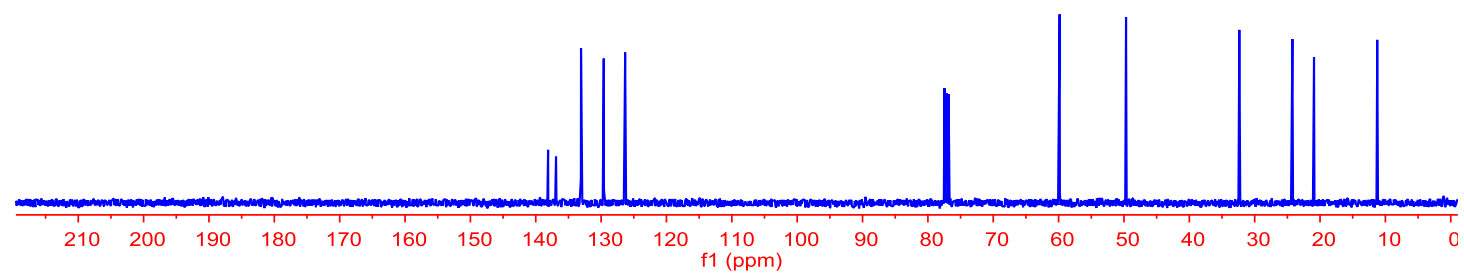




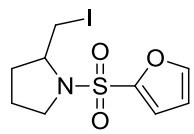

${ }^{1} \mathrm{H} \mathrm{NMR}\left(400 \mathrm{MHz}, \mathrm{CDCl}_{3}\right)$ of $\mathbf{4 m}$

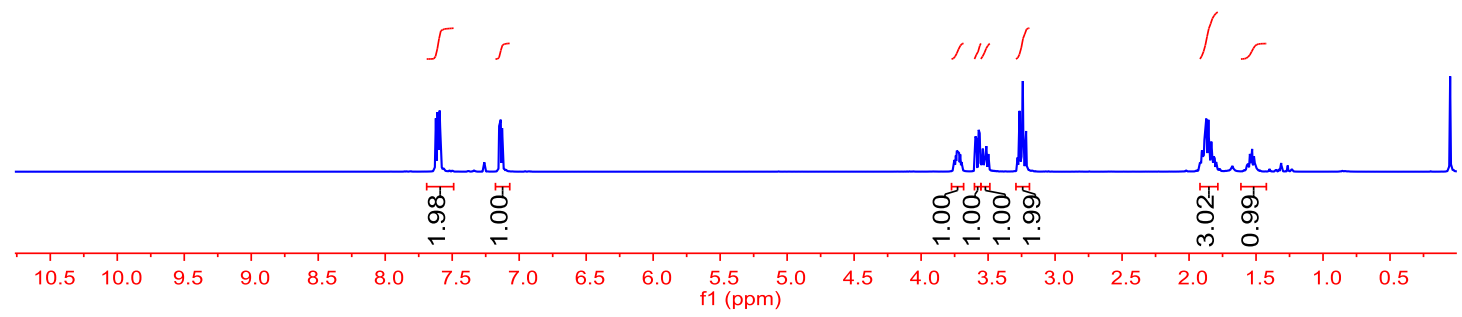

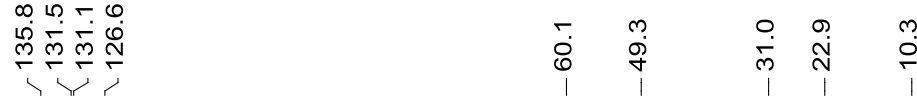

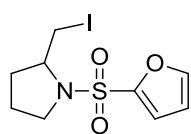

${ }^{13} \mathrm{C}$ NMR $\left(100 \mathrm{MHz}, \mathrm{CDCl}_{3}\right)$ of $\mathbf{4 m}$

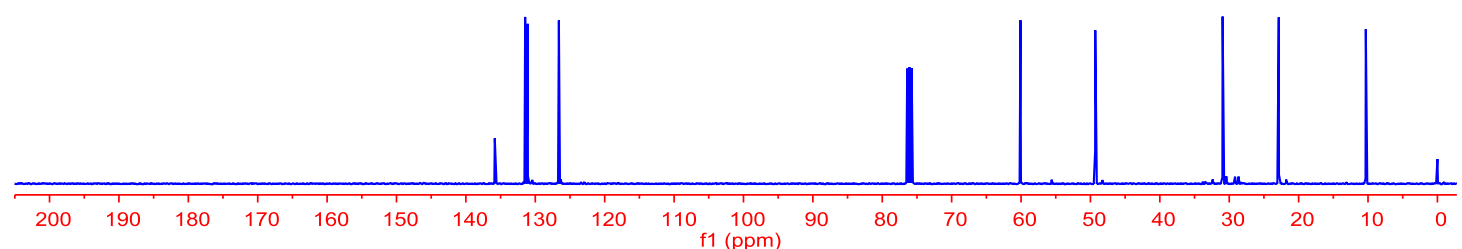




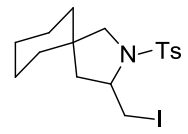

${ }^{1} \mathrm{H} \mathrm{NMR}\left(400 \mathrm{MHz}, \mathrm{CDCl}_{3}\right)$ of $\mathbf{4 n}$

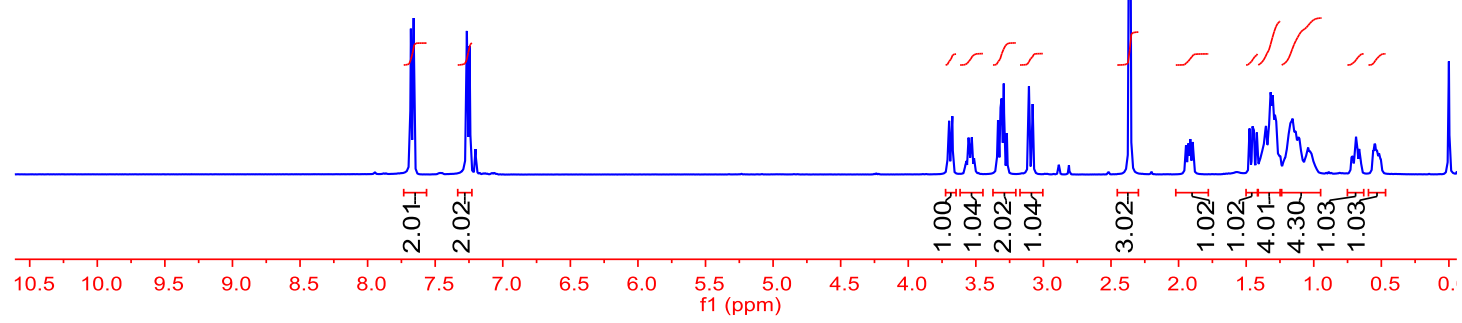

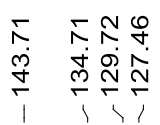

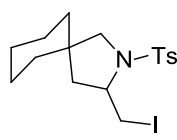

${ }^{13} \mathrm{C} \mathrm{NMR}\left(100 \mathrm{MHz}, \mathrm{CDCl}_{3}\right)$ of $\mathbf{4 n}$

\section{mำ

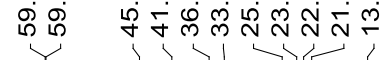

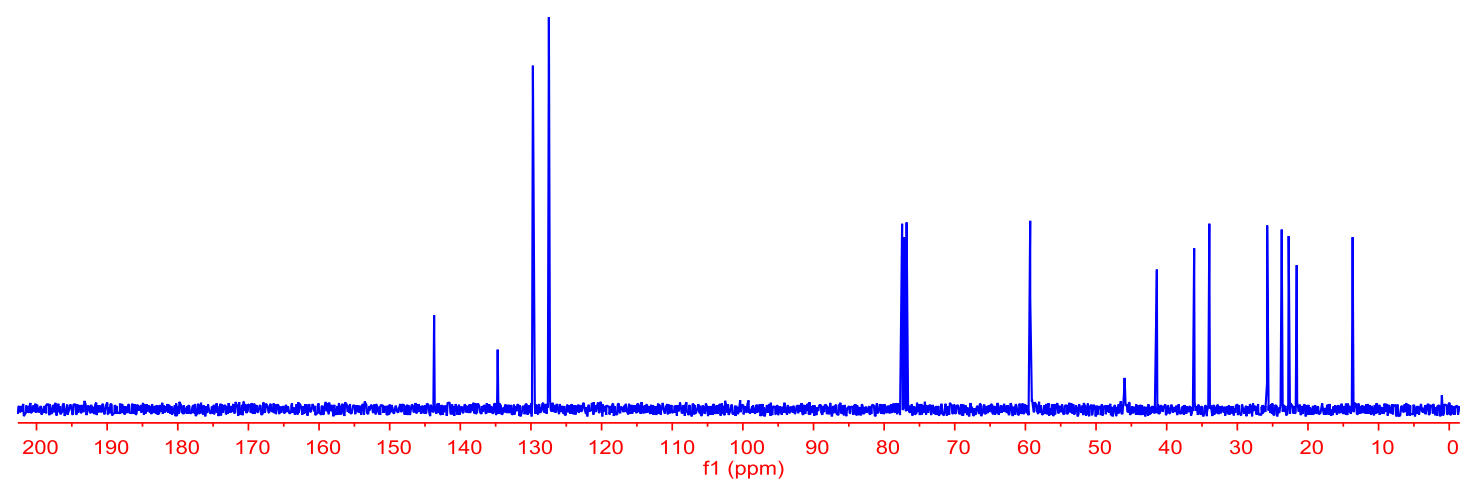




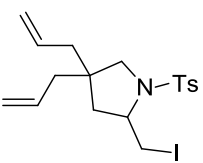

${ }^{1} \mathrm{H} \mathrm{NMR}\left(400 \mathrm{MHz}, \mathrm{CDCl}_{3}\right)$ of 40

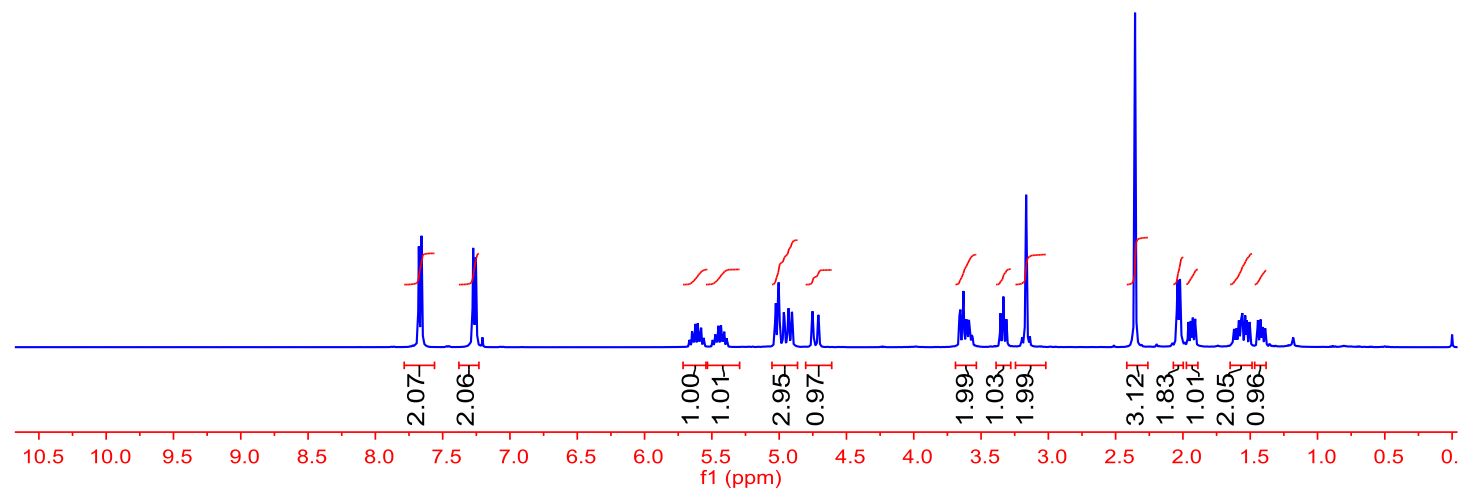

0

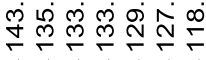

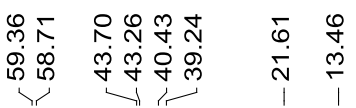

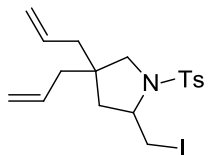

${ }^{13} \mathrm{H} \mathrm{NMR}\left(100 \mathrm{MHz}, \mathrm{CDCl}_{3}\right)$ of 40

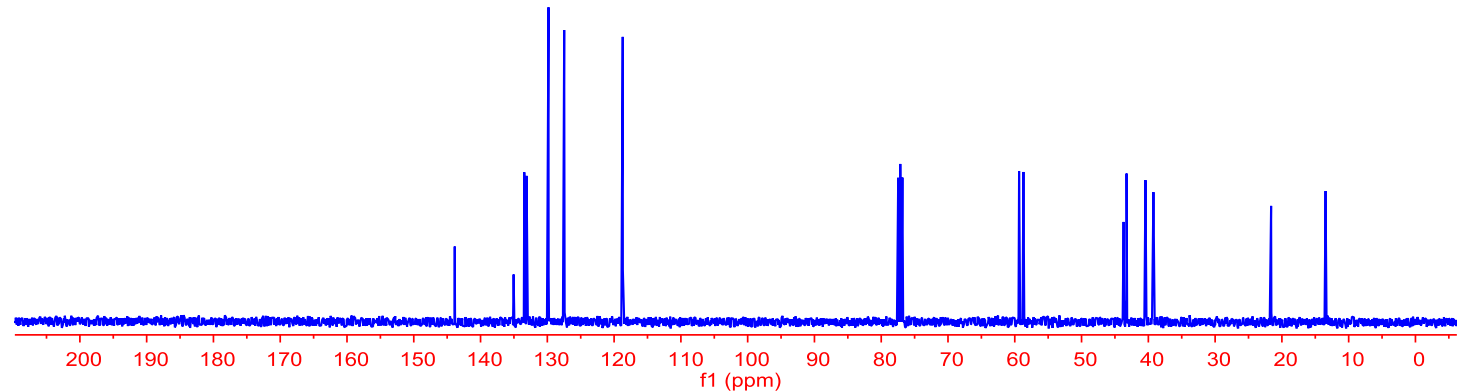




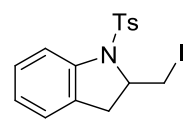

${ }^{1} \mathrm{H}$ NMR $\left(400 \mathrm{MHz}, \mathrm{CDCl}_{3}\right)$ of $4 \mathrm{p}$

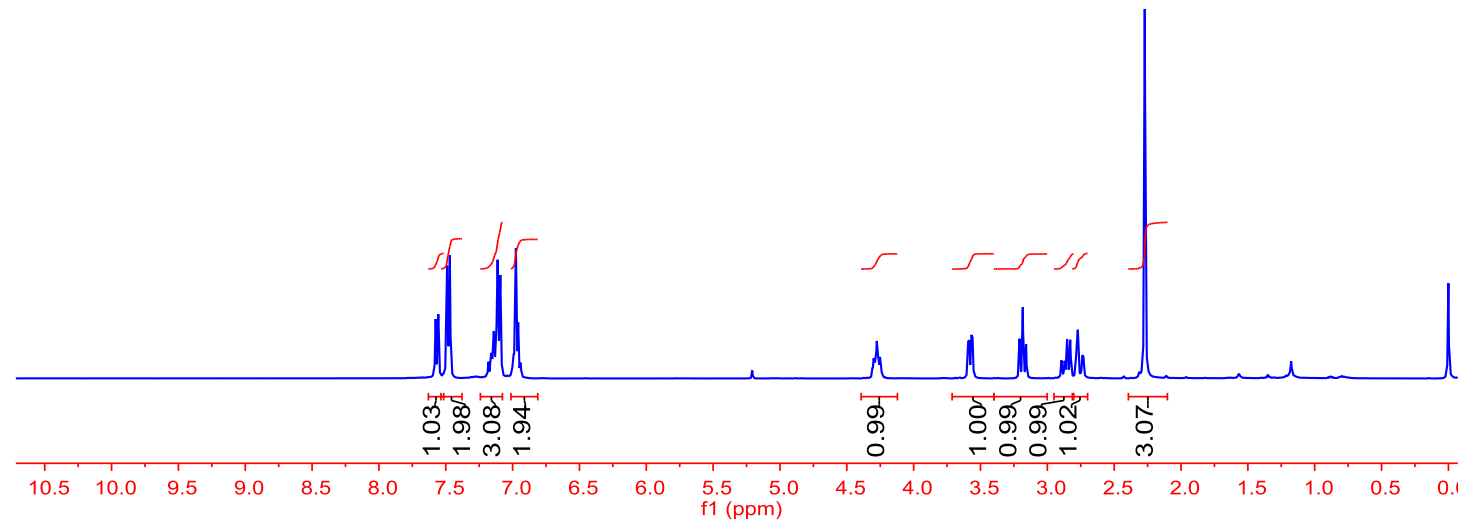

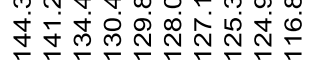

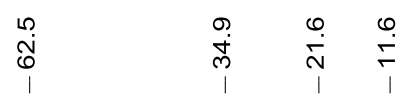

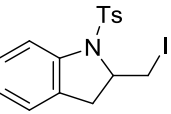

${ }^{13} \mathrm{C}$ NMR $\left(100 \mathrm{MHz}, \mathrm{CDCl}_{3}\right.$ ) of $4 p$

$200 \quad 190 \quad 180 \quad 170 \quad 160$

$\begin{array}{llll}50 & 140 & 130 & 120\end{array}$

$10 \quad 100 \quad 90$

$80 \quad 70$

$60 \quad 50$

40

$30 \quad 20$

10 

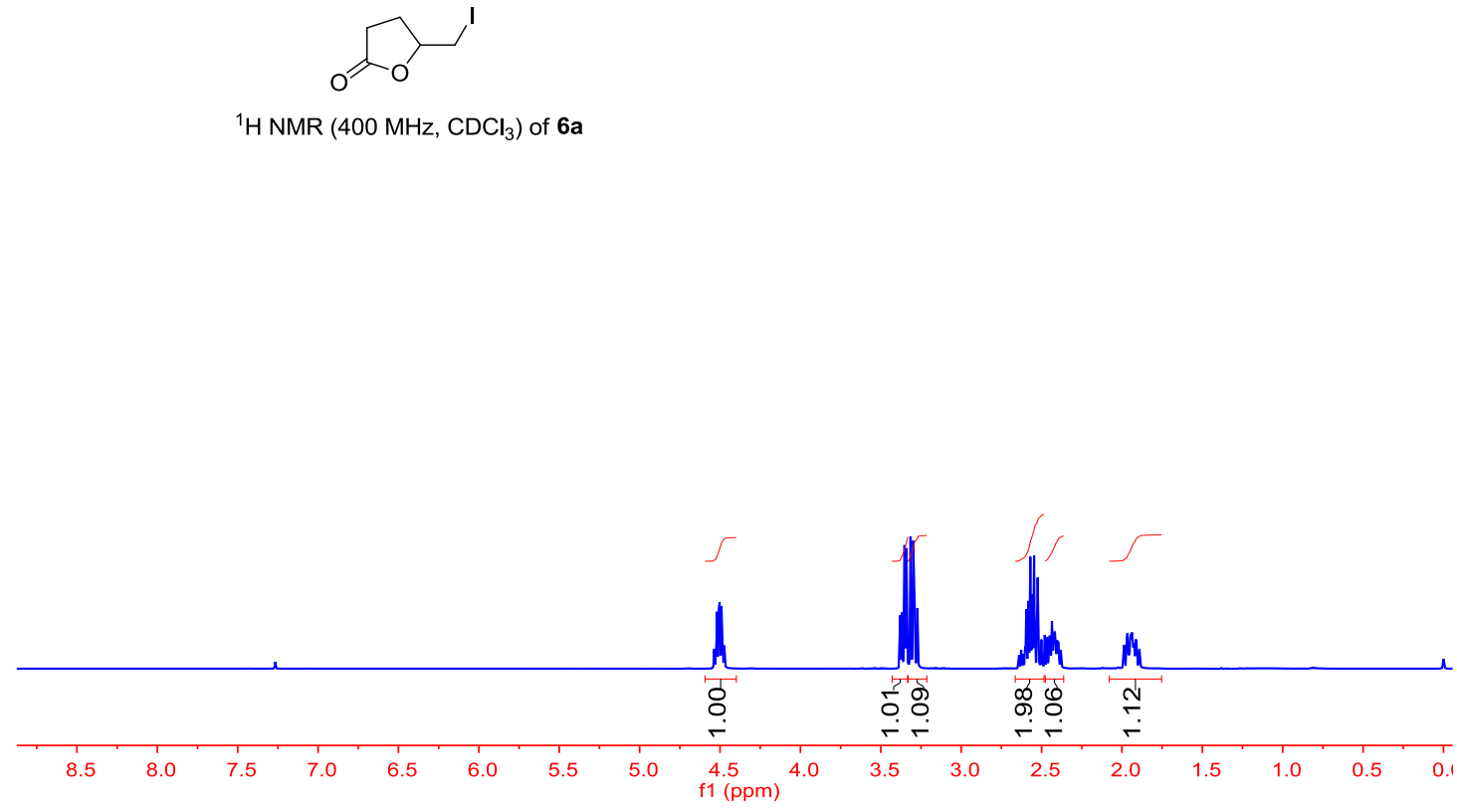

$\stackrel{m}{n}$

$\stackrel{\infty}{\infty}$

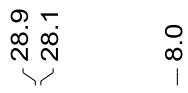

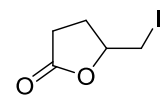

${ }^{13} \mathrm{C} \mathrm{NMR}\left(100 \mathrm{MHz}, \mathrm{CDCl}_{3}\right)$ of $6 \mathbf{a}$

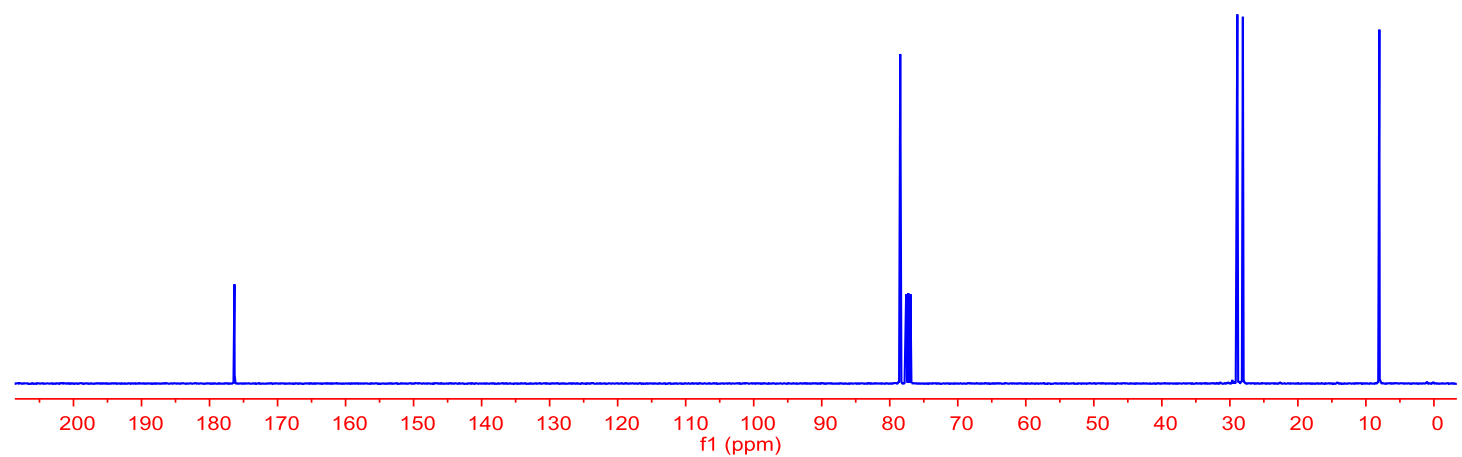




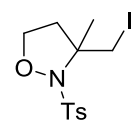

${ }^{1} \mathrm{H} \mathrm{NMR}\left(400 \mathrm{MHz}, \mathrm{CDCl}_{3}\right)$ of $\mathbf{6 b}$

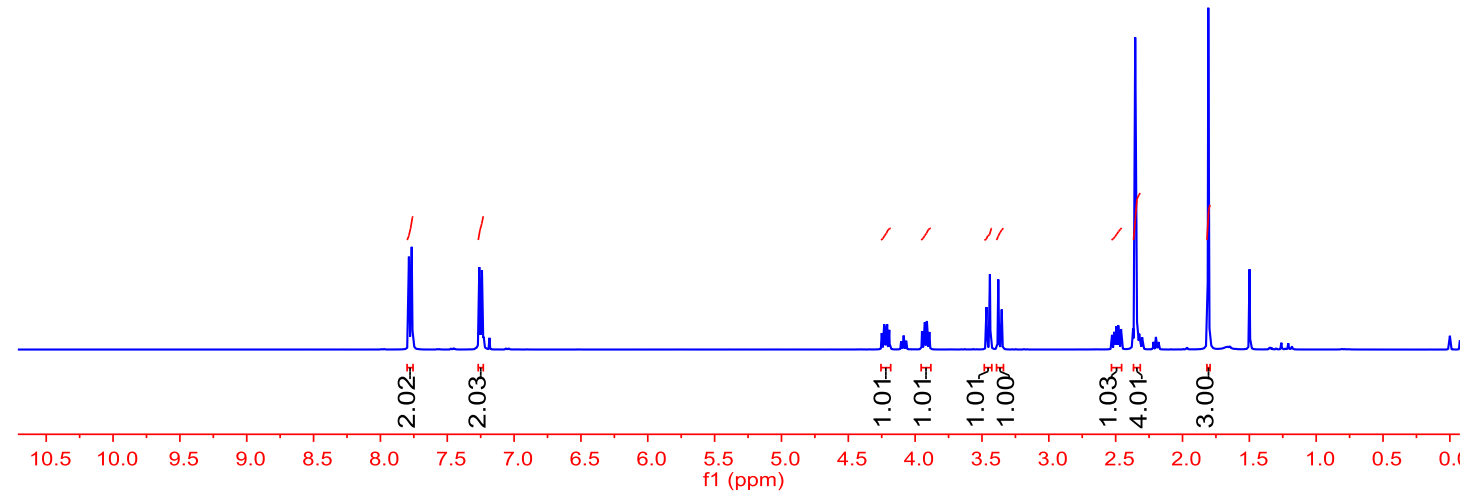

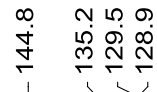

m)

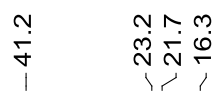

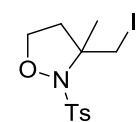

${ }^{13} \mathrm{C} \mathrm{NMR} \mathrm{(100} \mathrm{MHz,} \mathrm{CDCl}_{3}$ ) of $\mathbf{6 b}$

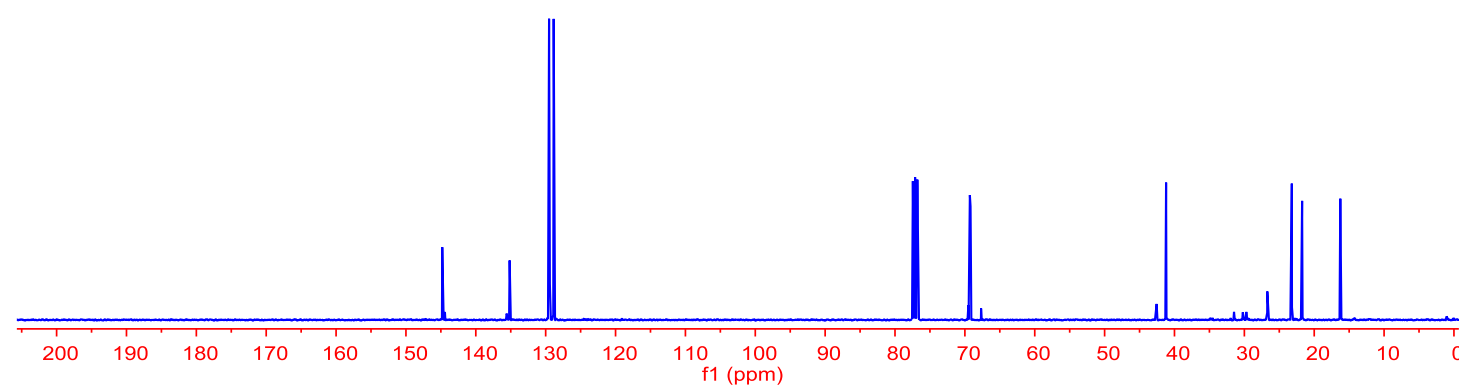




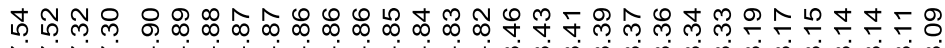

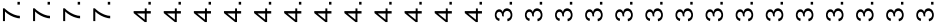

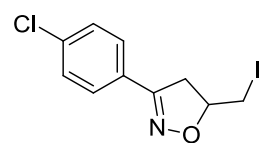

${ }^{1} \mathrm{H}$ NMR $\left(400 \mathrm{MHz}, \mathrm{CDCl}_{3}\right)$ of $6 \mathrm{c}$
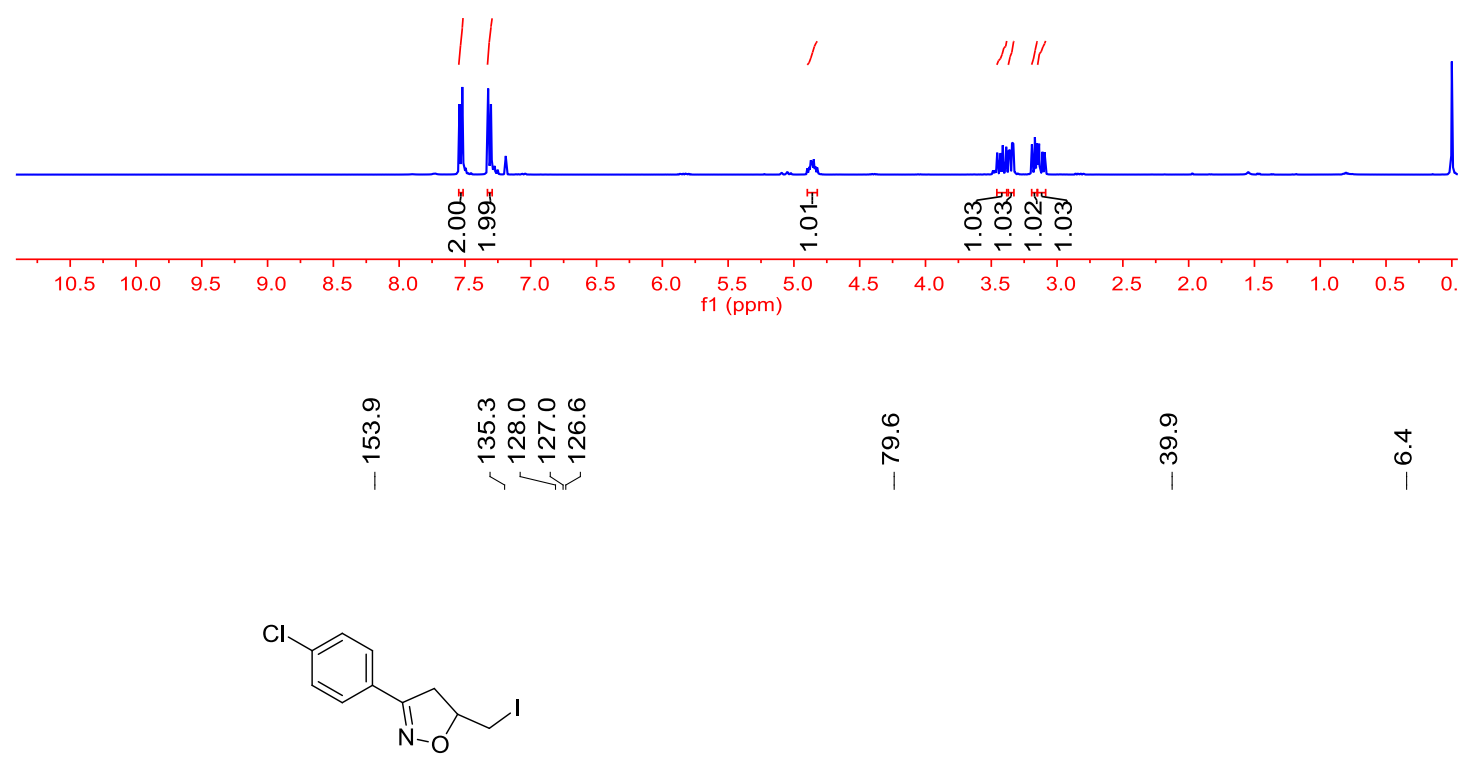

${ }^{13} \mathrm{C}$ NMR (100 MHz, $\mathrm{CDCl}_{3}$ ) of $6 \mathrm{c}$

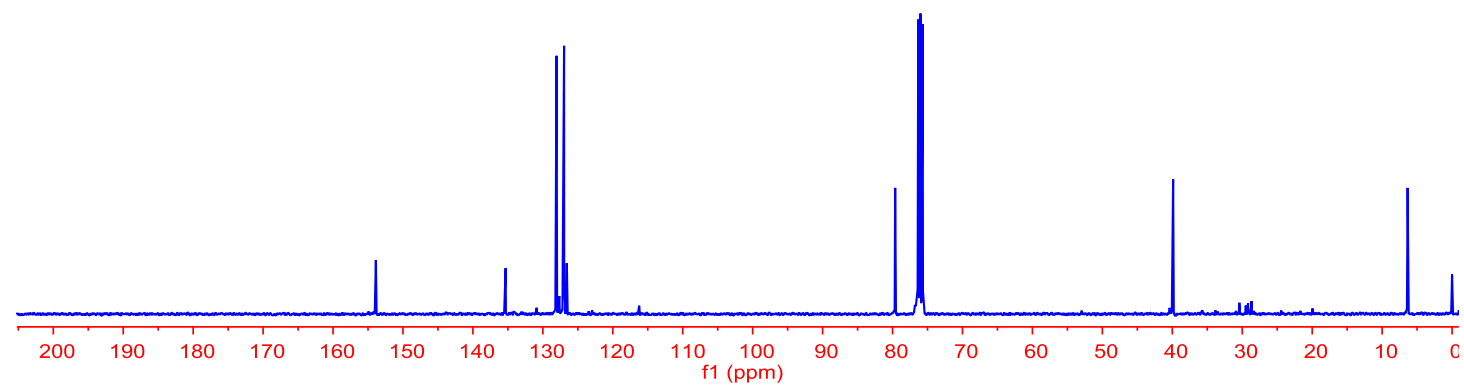




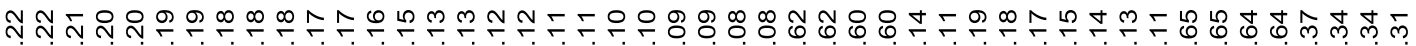

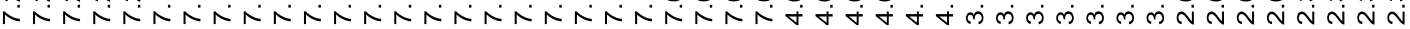

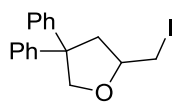

${ }^{1} \mathrm{H}$ NMR $\left(400 \mathrm{MHz}, \mathrm{CDCl}_{3}\right)$ of $\mathbf{6 d}$

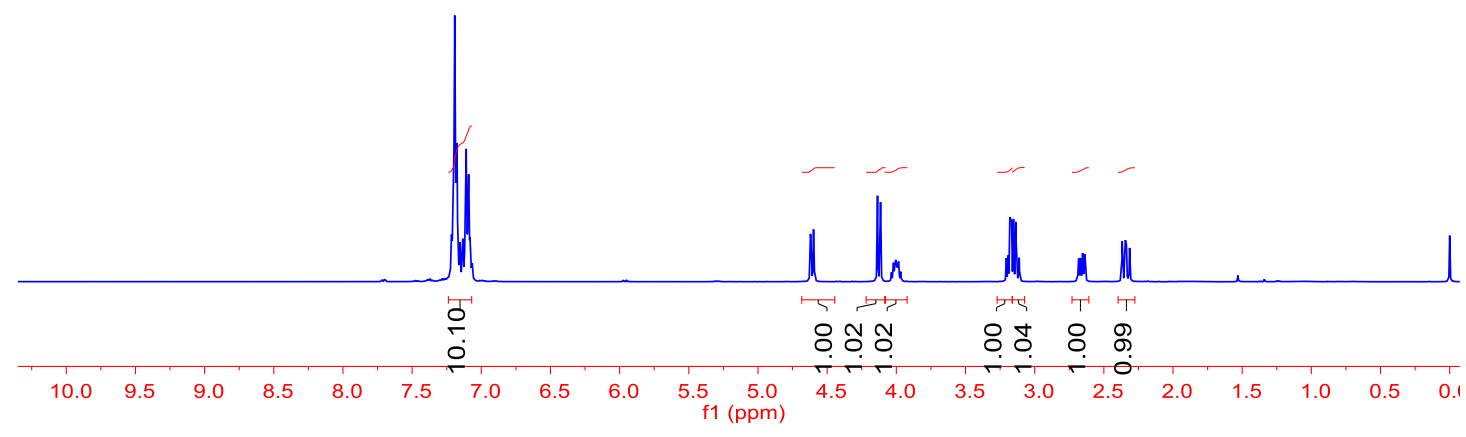

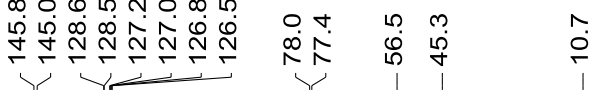

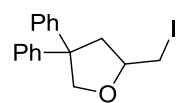

${ }^{13} \mathrm{C}$ NMR $\left(100 \mathrm{MHz}, \mathrm{CDCl}_{3}\right)$ of $\mathbf{6 d}$

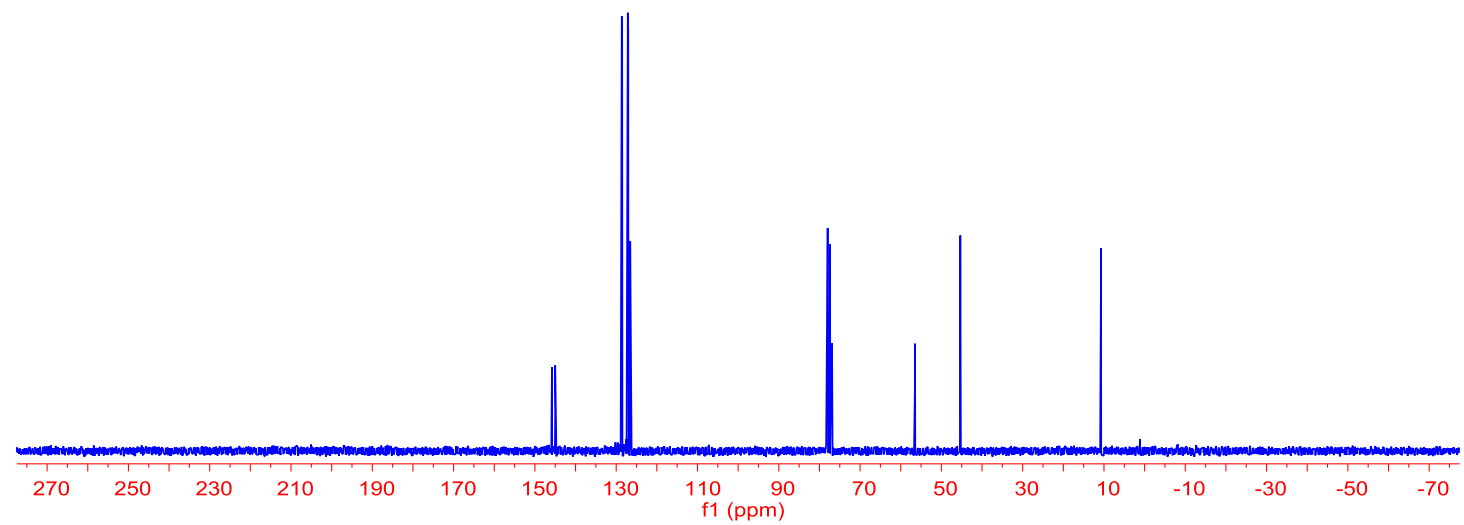




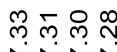



NN

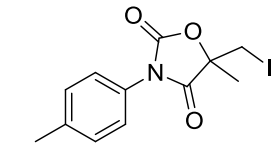

${ }^{1} \mathrm{H}$ NMR $\left(400 \mathrm{MHz}, \mathrm{CDCl}_{3}\right)$ of $6 \mathbf{e}$
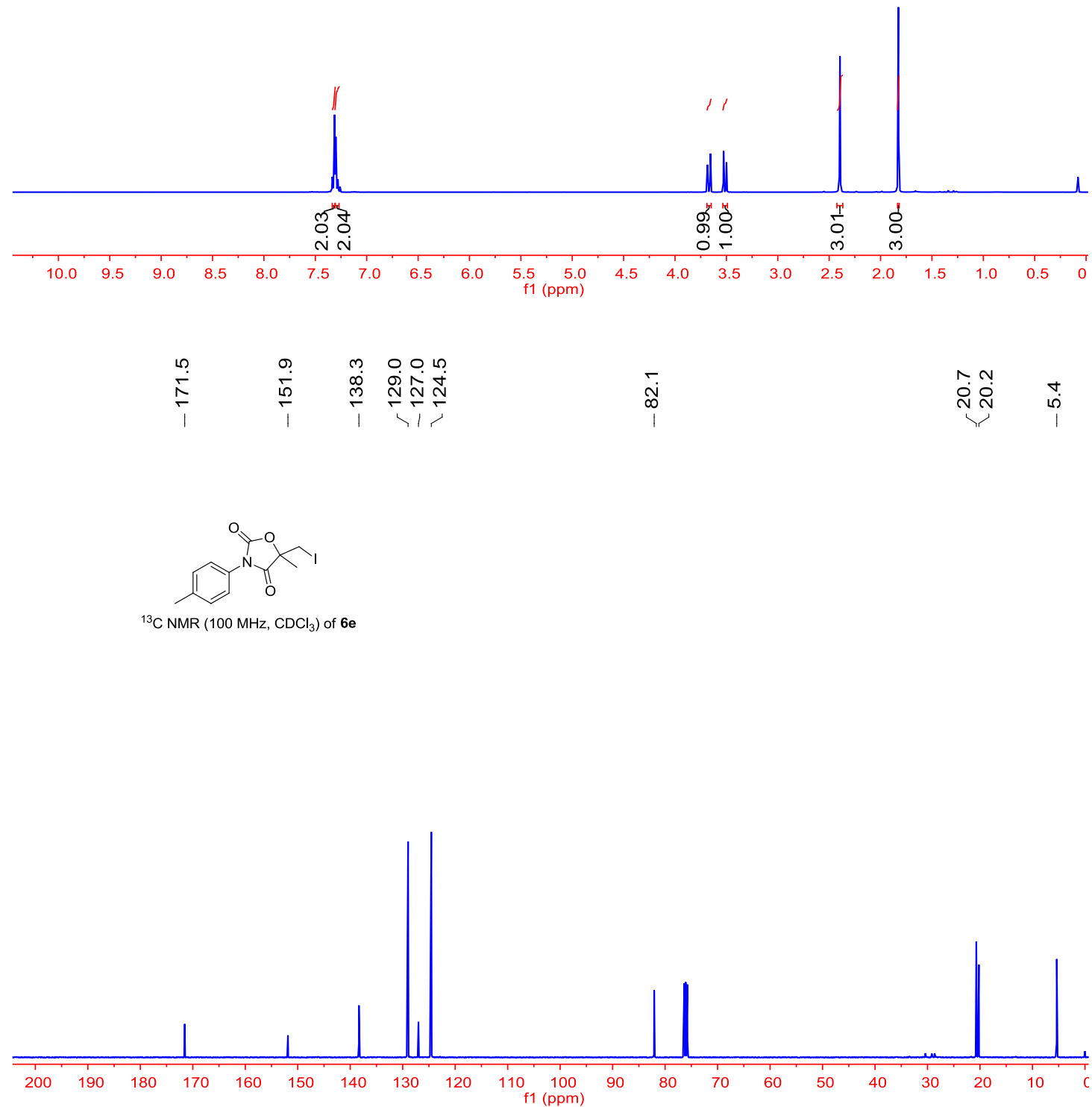


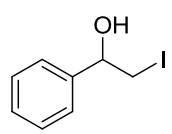

${ }^{1} \mathrm{H}$ NMR $\left(400 \mathrm{MHz}, \mathrm{CDCl}_{3}\right)$ of $8 \mathbf{a}$
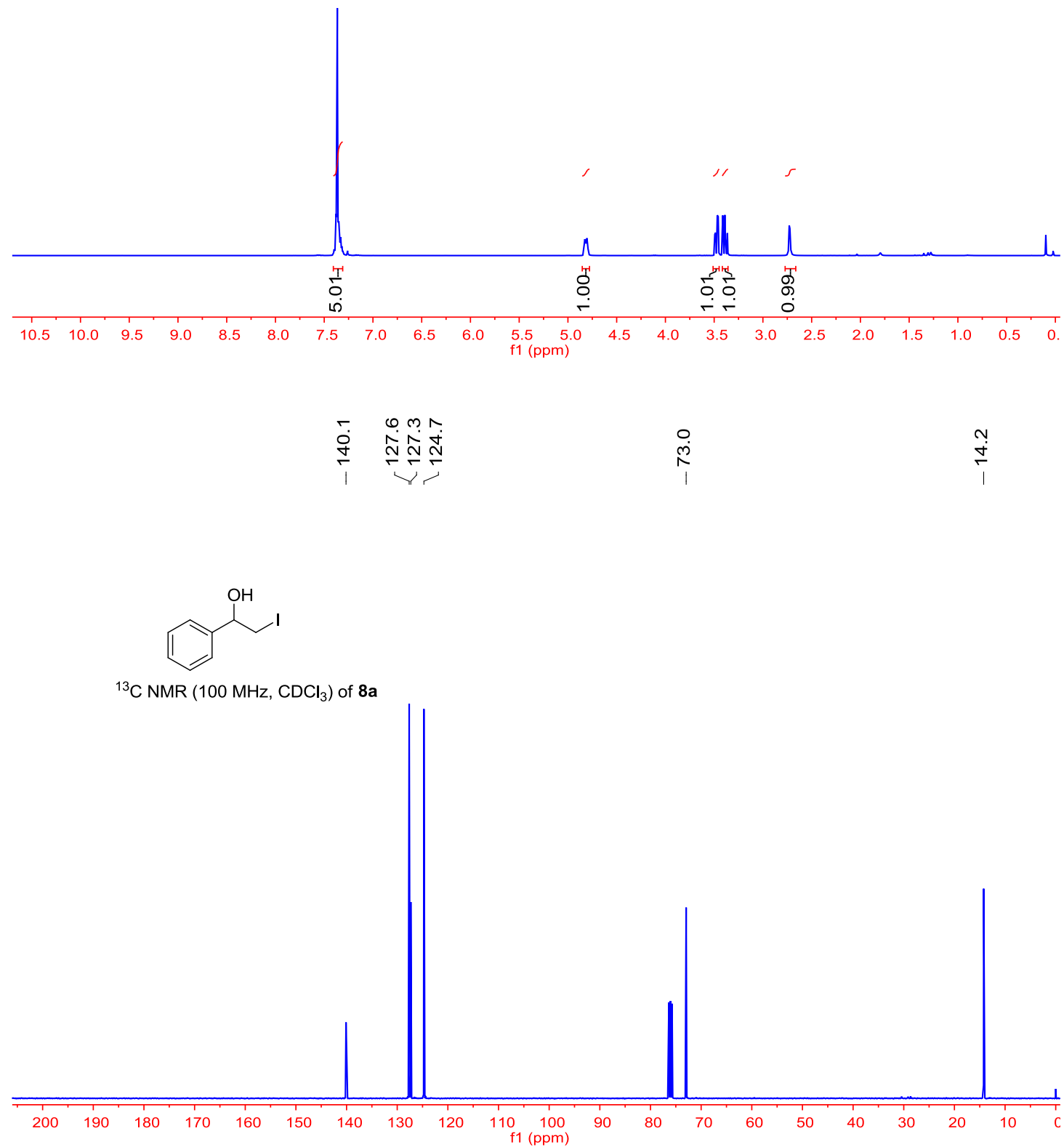


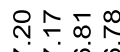

ก

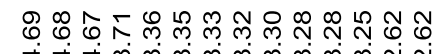

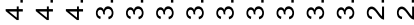

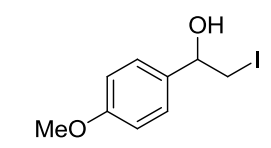

${ }^{1} \mathrm{H} \mathrm{NMR}\left(400 \mathrm{MHz}, \mathrm{CDCl}_{3}\right)$ of $\mathbf{8 b}$

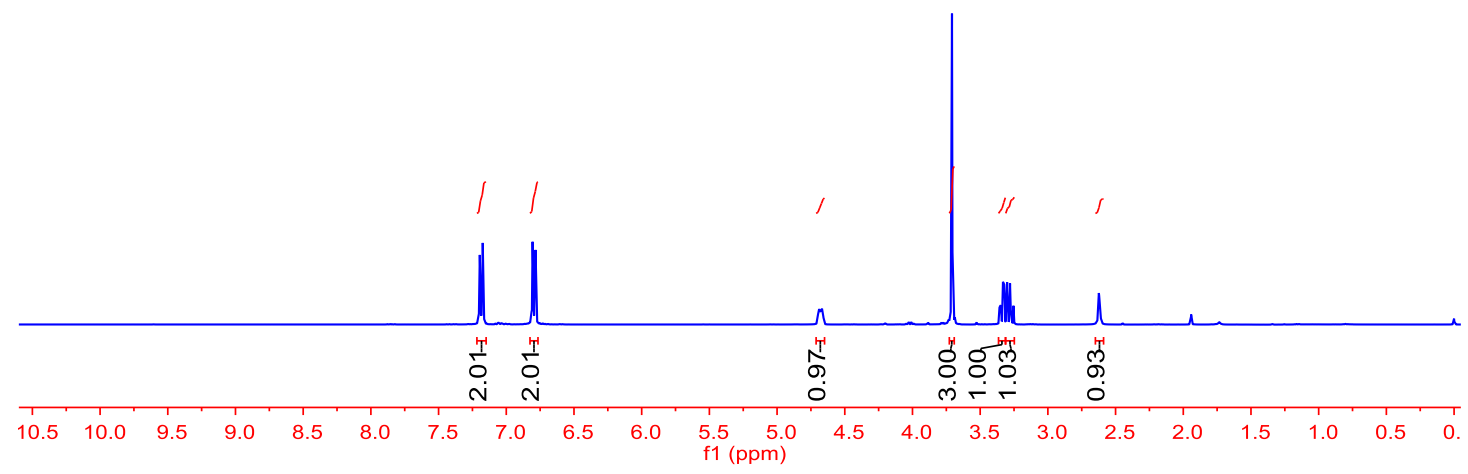

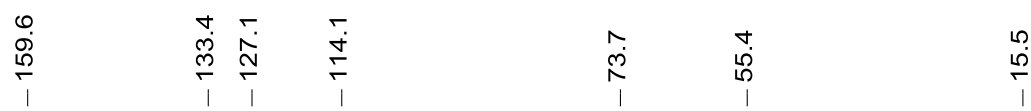

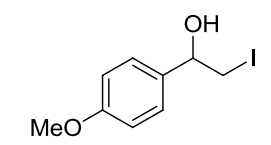

${ }^{13} \mathrm{C} \mathrm{NMR}\left(100 \mathrm{MHz}, \mathrm{CDCl}_{3}\right)$ of $\mathbf{8 b}$

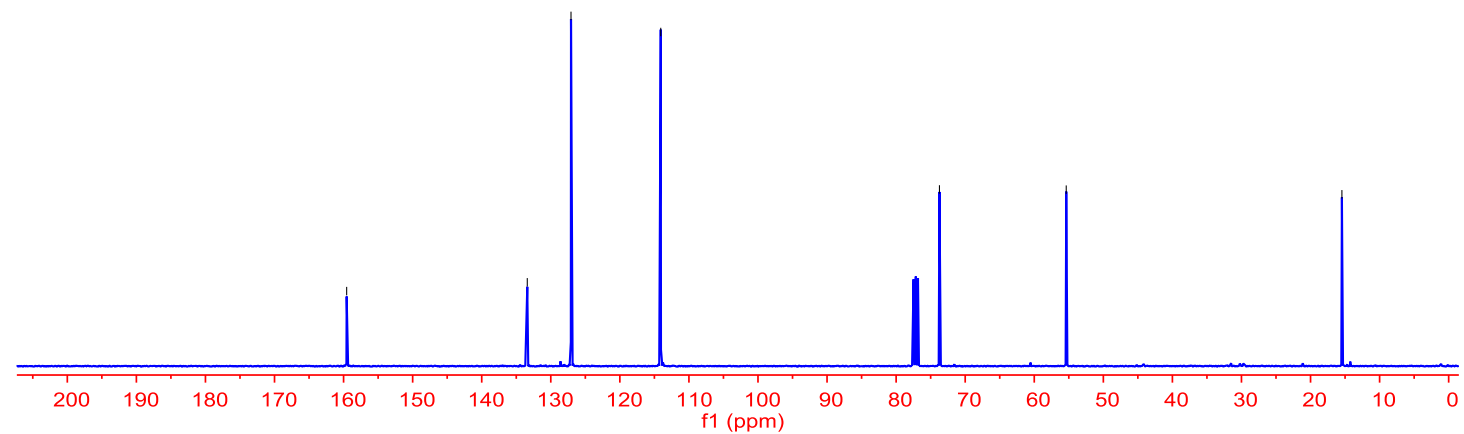




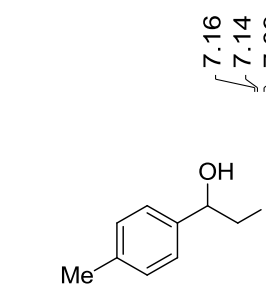

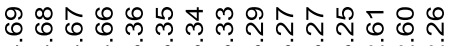

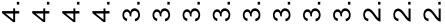

${ }^{1} \mathrm{H}$ NMR $\left(400 \mathrm{MHz}, \mathrm{CDCl}_{3}\right)$ of $8 \mathrm{c}$

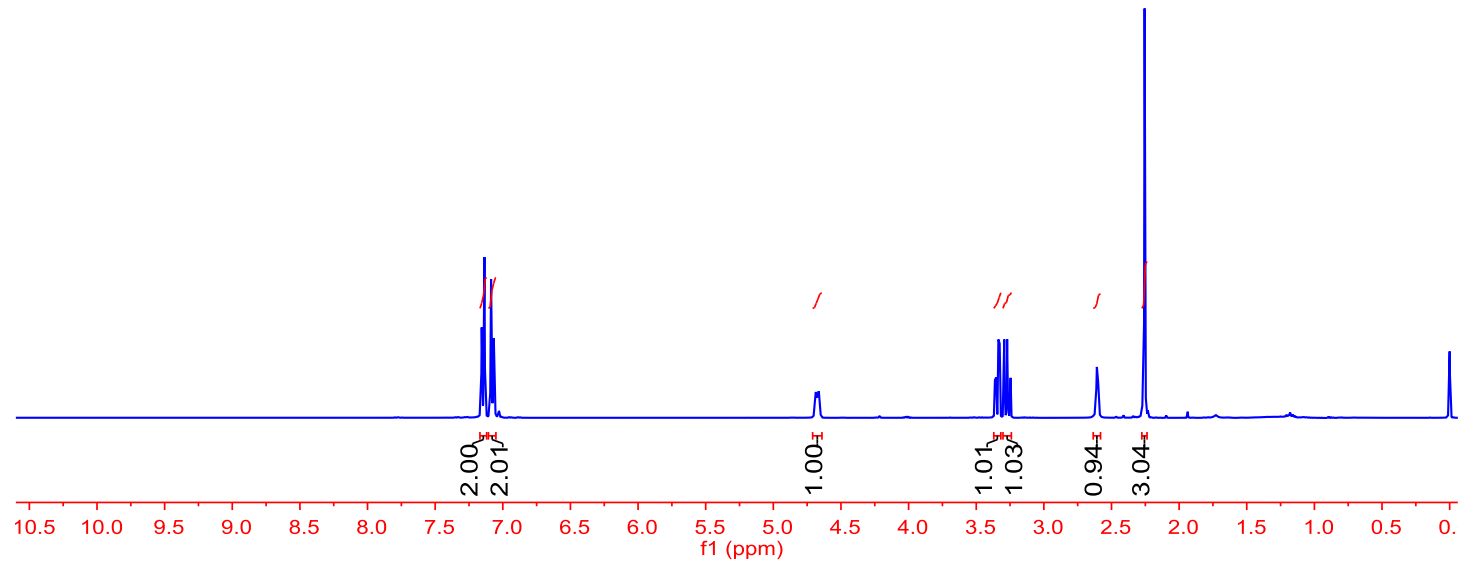

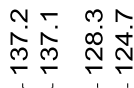

$\stackrel{\infty}{N}$

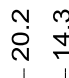

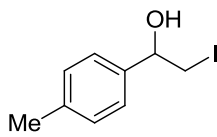

${ }^{13} \mathrm{C} \mathrm{NMR}\left(100 \mathrm{MHz}, \mathrm{CDCl}_{3}\right)$ of $8 \mathrm{c}$

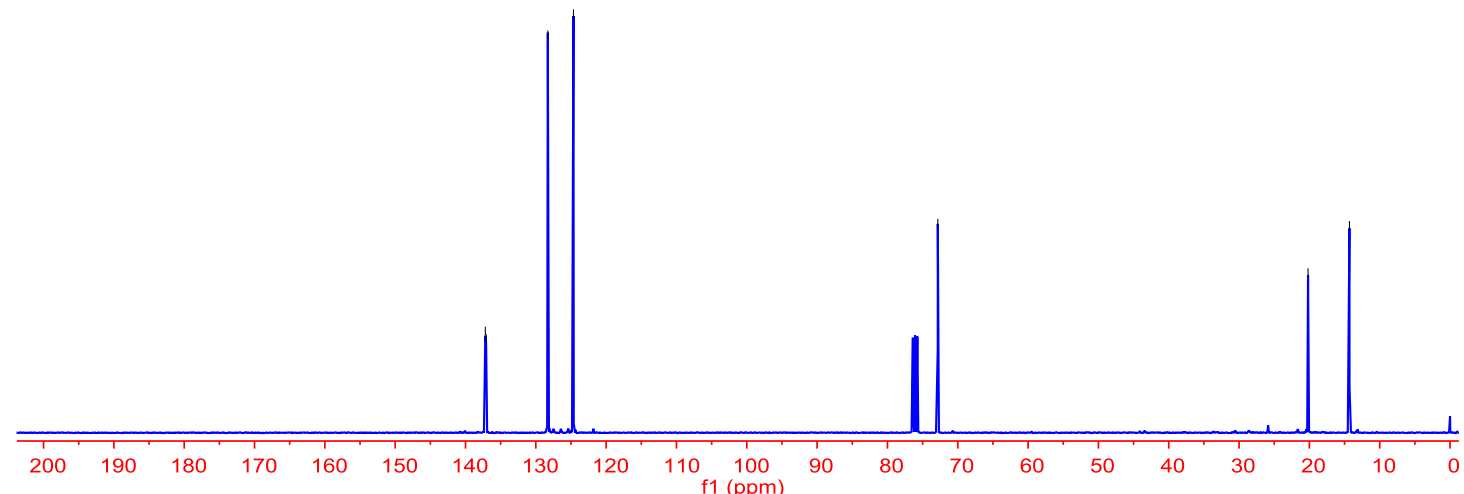




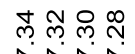

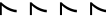

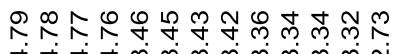

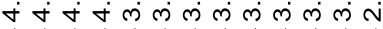

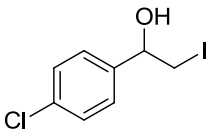

${ }^{1} \mathrm{H}$ NMR $\left(400 \mathrm{MHz}, \mathrm{CDCl}_{3}\right)$ of $\mathbf{8 d}$

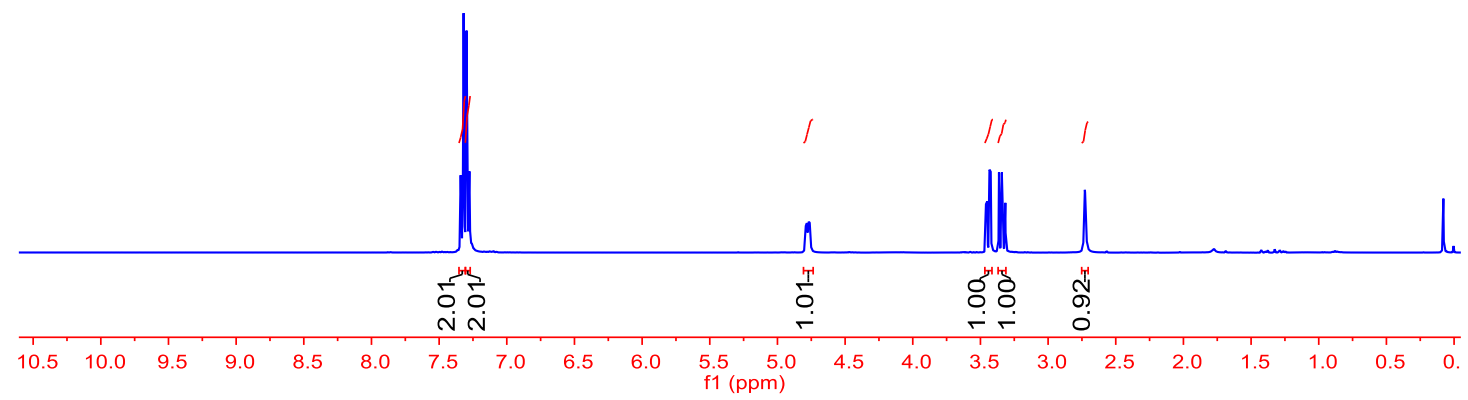

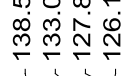

$\stackrel{\substack{1 \\ N}}{\sqrt{n}}$

$\stackrel{\oplus}{\stackrel{\leftrightarrow}{+}}$

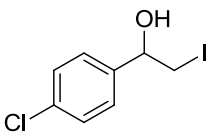

${ }^{13} \mathrm{C} \mathrm{NMR}\left(100 \mathrm{MHz}, \mathrm{CDCl}_{3}\right.$ ) of $\mathbf{8 d}$

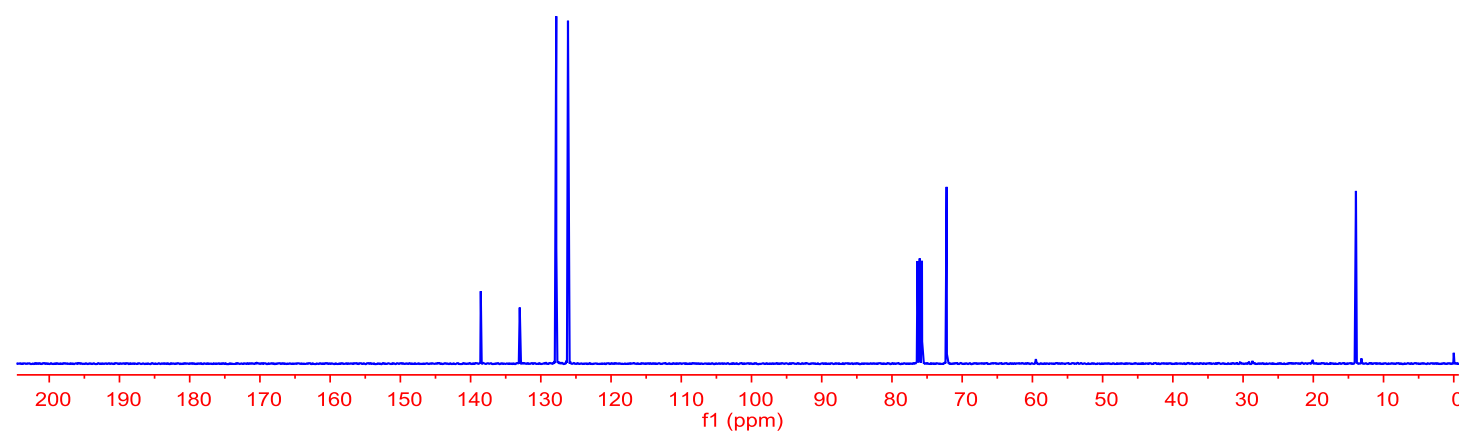




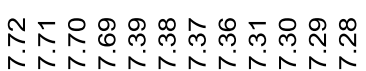

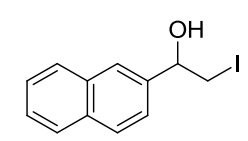

${ }^{1} \mathrm{H}$ NMR $\left(400 \mathrm{MHz}, \mathrm{CDCl}_{3}\right)$ of $8 \mathbf{e}$

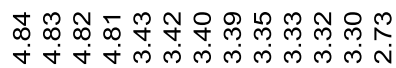
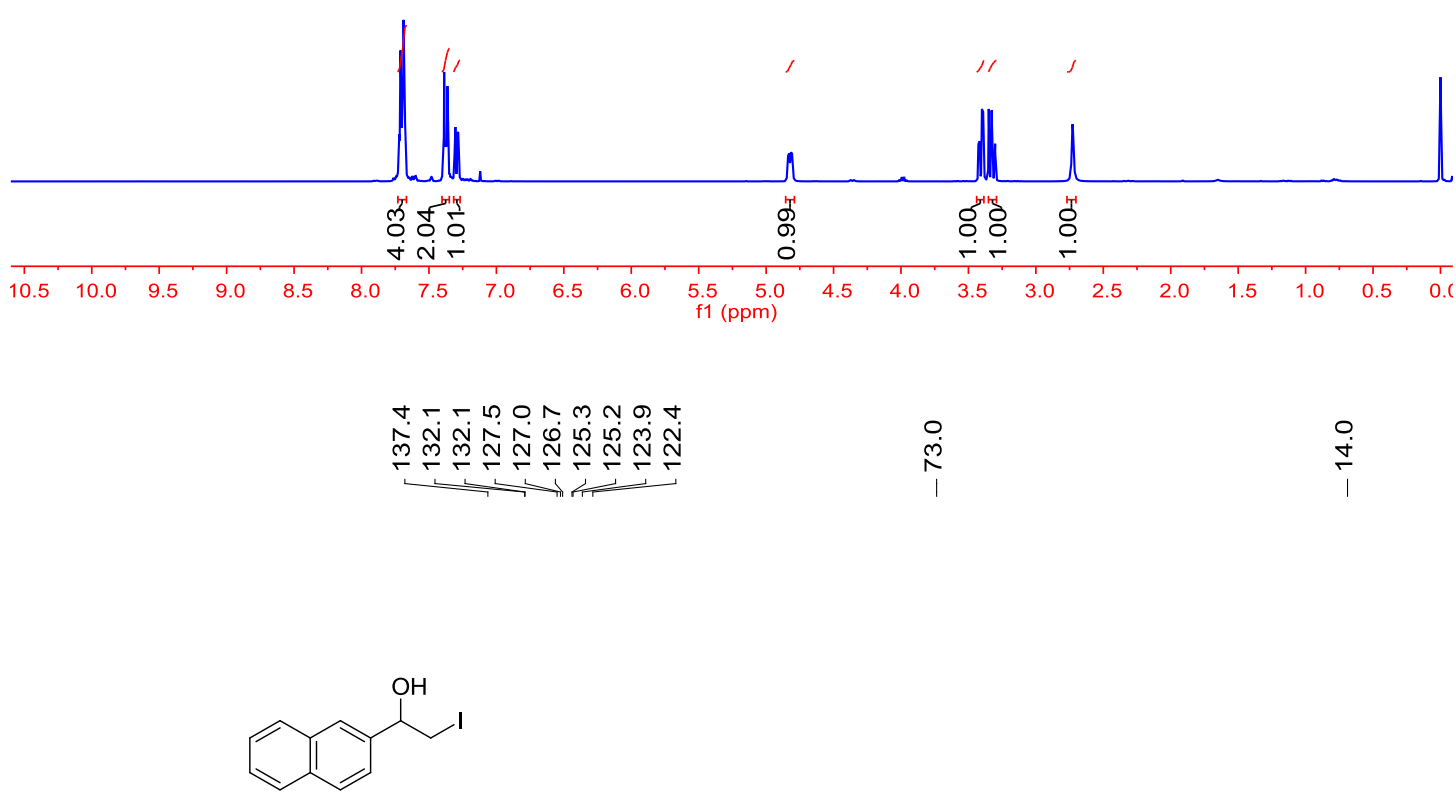

${ }^{13} \mathrm{C} \mathrm{NMR}\left(100 \mathrm{MHz}, \mathrm{CDCl}_{3}\right)$ of $8 \mathbf{e}$

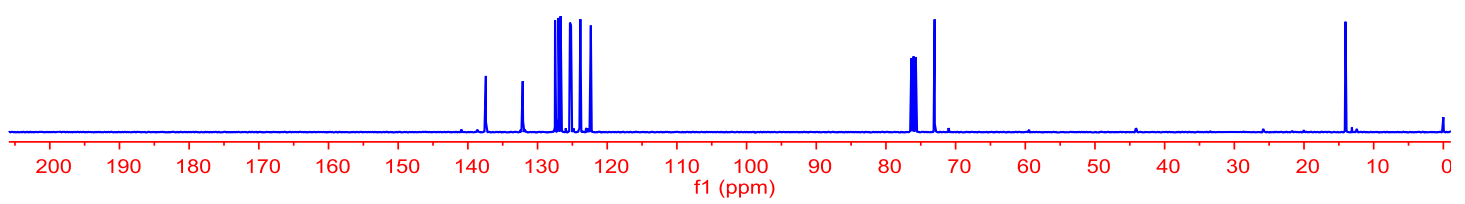

S69 


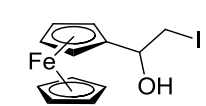

${ }^{1} \mathrm{H} \mathrm{NMR}\left(400 \mathrm{MHz}, \mathrm{CDCl}_{3}\right)$ of $8 \mathrm{f}$

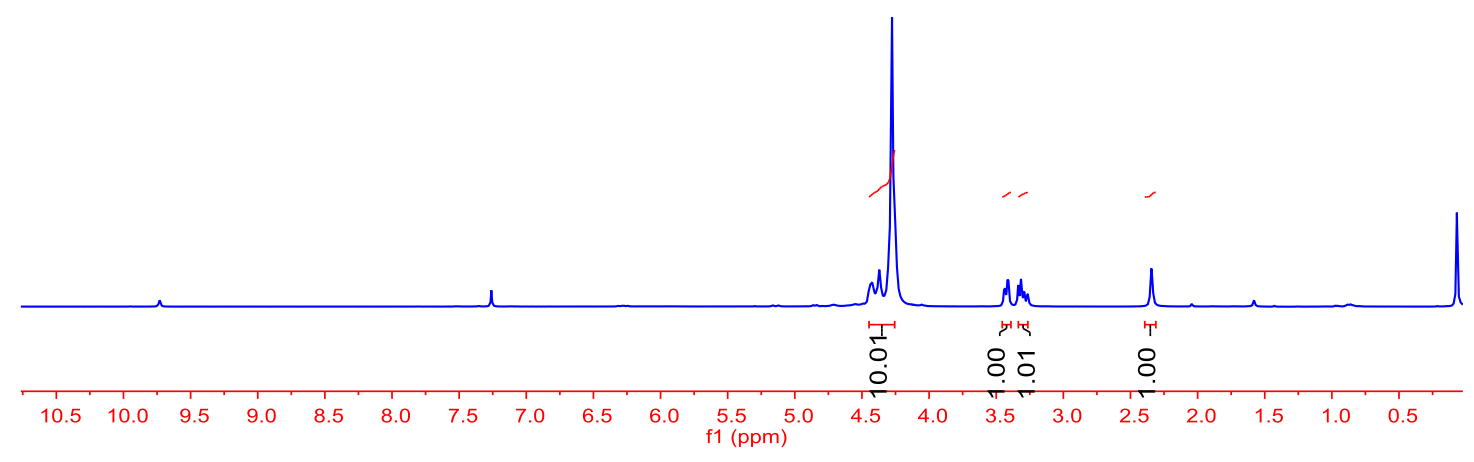

$\sigma \infty N \infty \infty \infty$

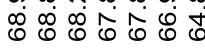

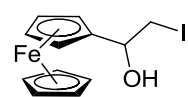

${ }^{13} \mathrm{C} \mathrm{NMR} \mathrm{(100} \mathrm{MHz,} \mathrm{CDCl}_{3}$ ) of 8 f

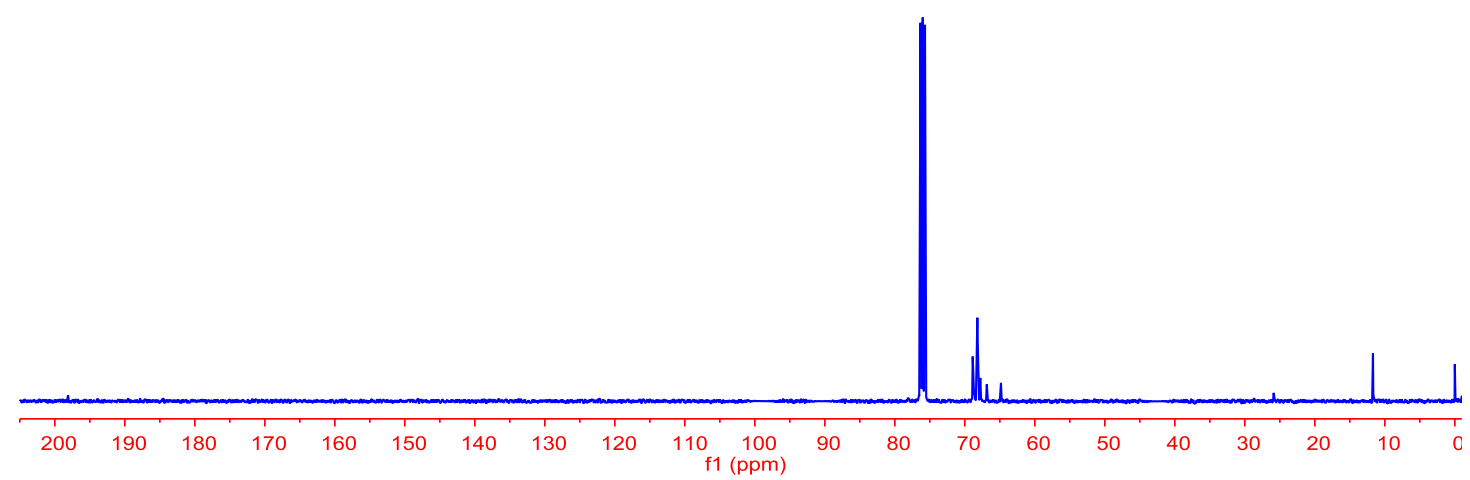




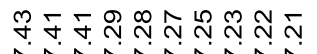

ヘNベNベNN

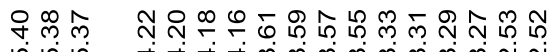

மं மं

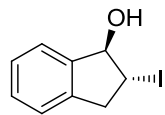

${ }^{1} \mathrm{H} \mathrm{NMR}\left(400 \mathrm{MHz}, \mathrm{CDCl}_{3}\right)$ of $\mathbf{8 g}$

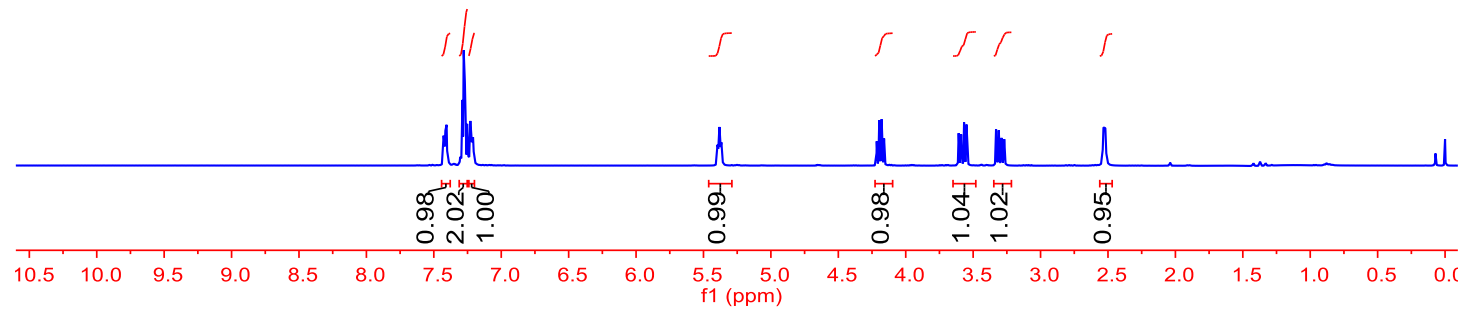

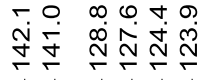

0
$\substack{\infty \\ \infty \\ 1}$

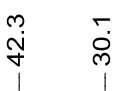

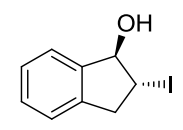

${ }^{13} \mathrm{C} \mathrm{NMR}\left(100 \mathrm{MHz}, \mathrm{CDCl}_{3}\right)$ of $\mathbf{8 g}$

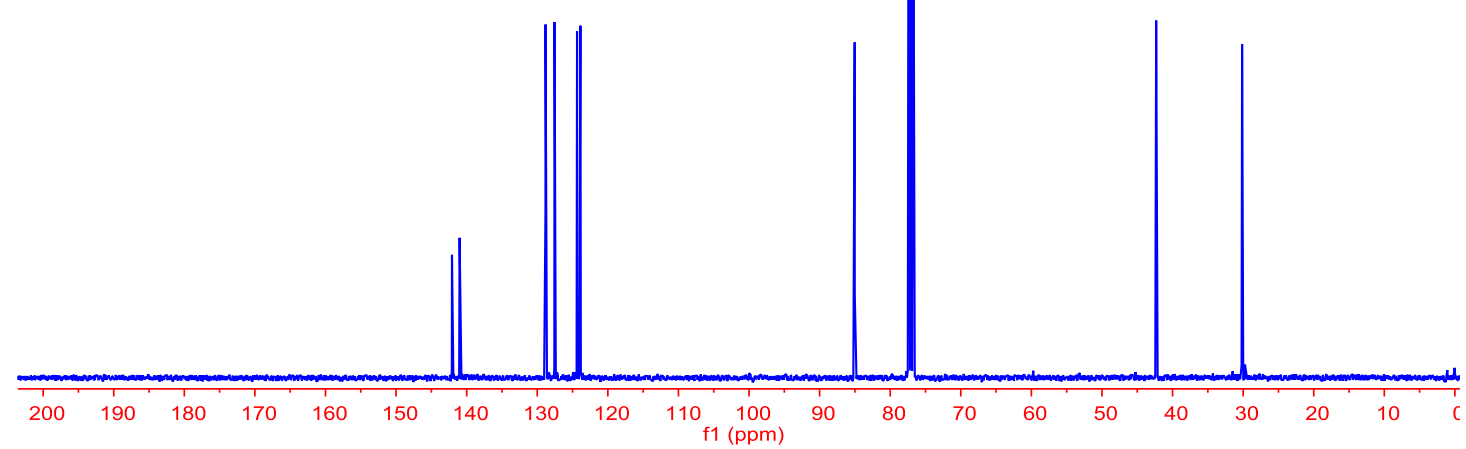




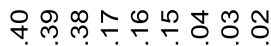

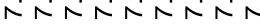

N

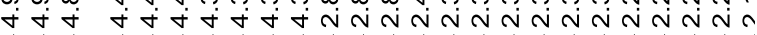

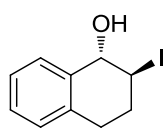

${ }^{1} \mathrm{H}$ NMR $\left(400 \mathrm{MHz}, \mathrm{CDCl}_{3}\right)$ of $\mathbf{8 h}$

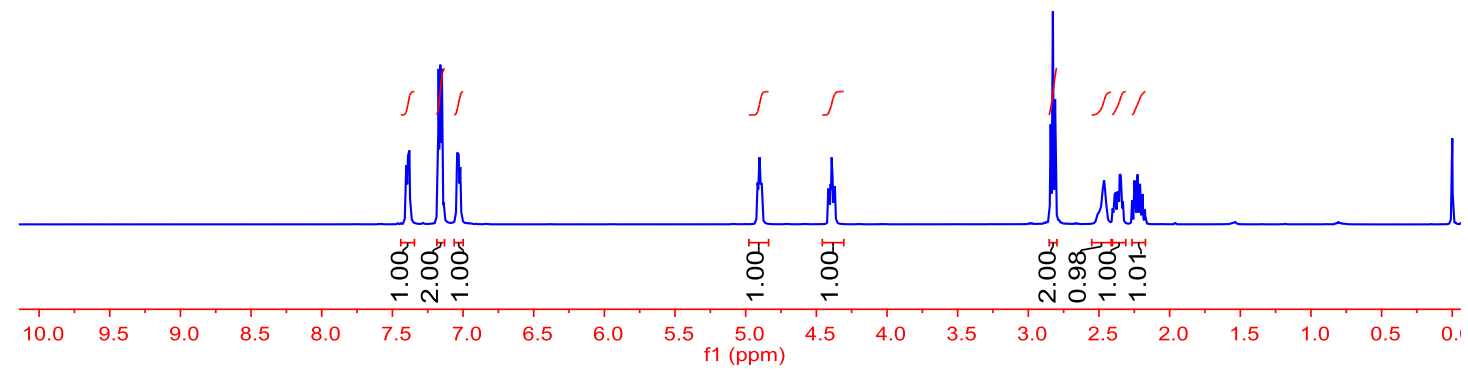

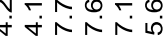

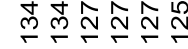
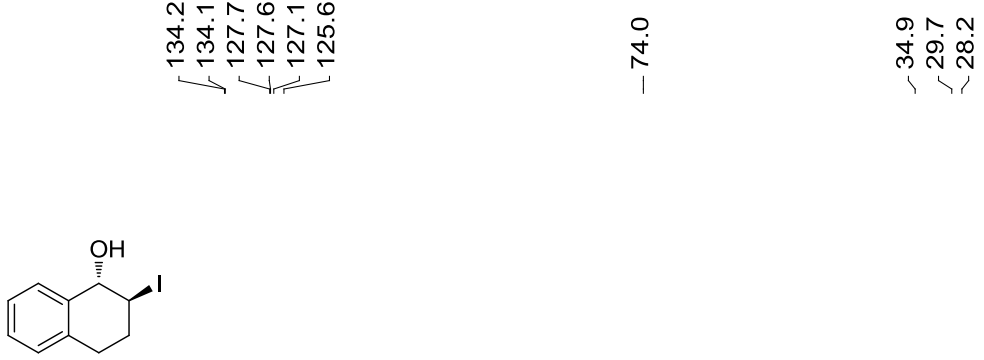

${ }^{13} \mathrm{C} \mathrm{NMR}\left(100 \mathrm{MHz}, \mathrm{CDCl}_{3}\right)$ of $\mathbf{8 h}$

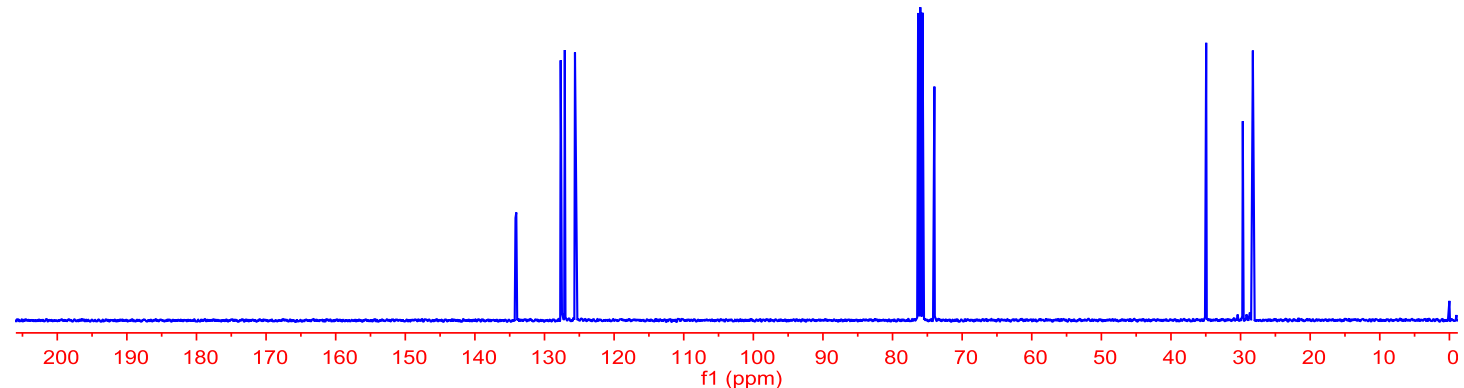




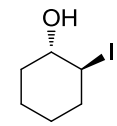

${ }^{1} \mathrm{H} \mathrm{NMR}\left(400 \mathrm{MHz}, \mathrm{CDCl}_{3}\right)$ of $8 \mathbf{i}$

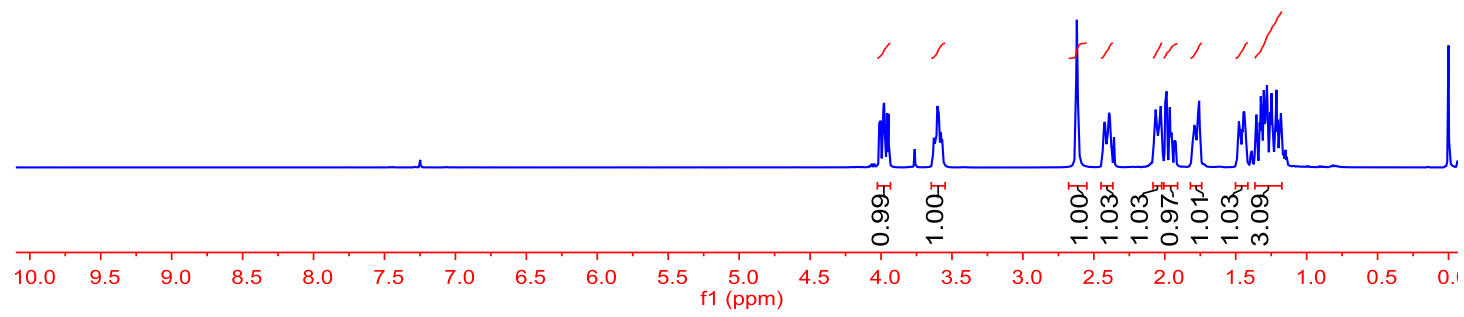

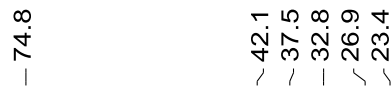

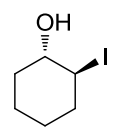

${ }^{13} \mathrm{C} \mathrm{NMR}\left(100 \mathrm{MHz}, \mathrm{CDCl}_{3}\right)$ of $8 \mathbf{i}$

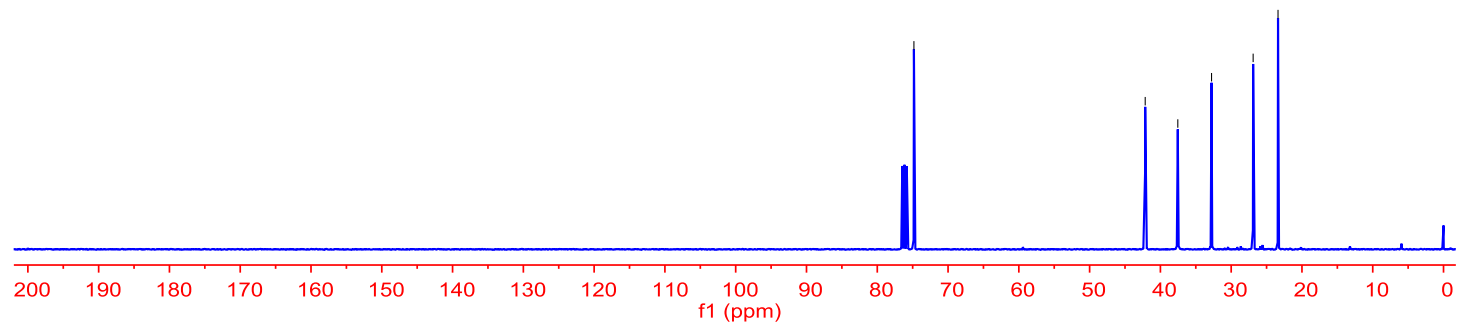




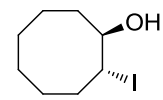

${ }^{1} \mathrm{H} \mathrm{NMR}\left(400 \mathrm{MHz}, \mathrm{CDCl}_{3}\right)$ of $\mathbf{8 j}$
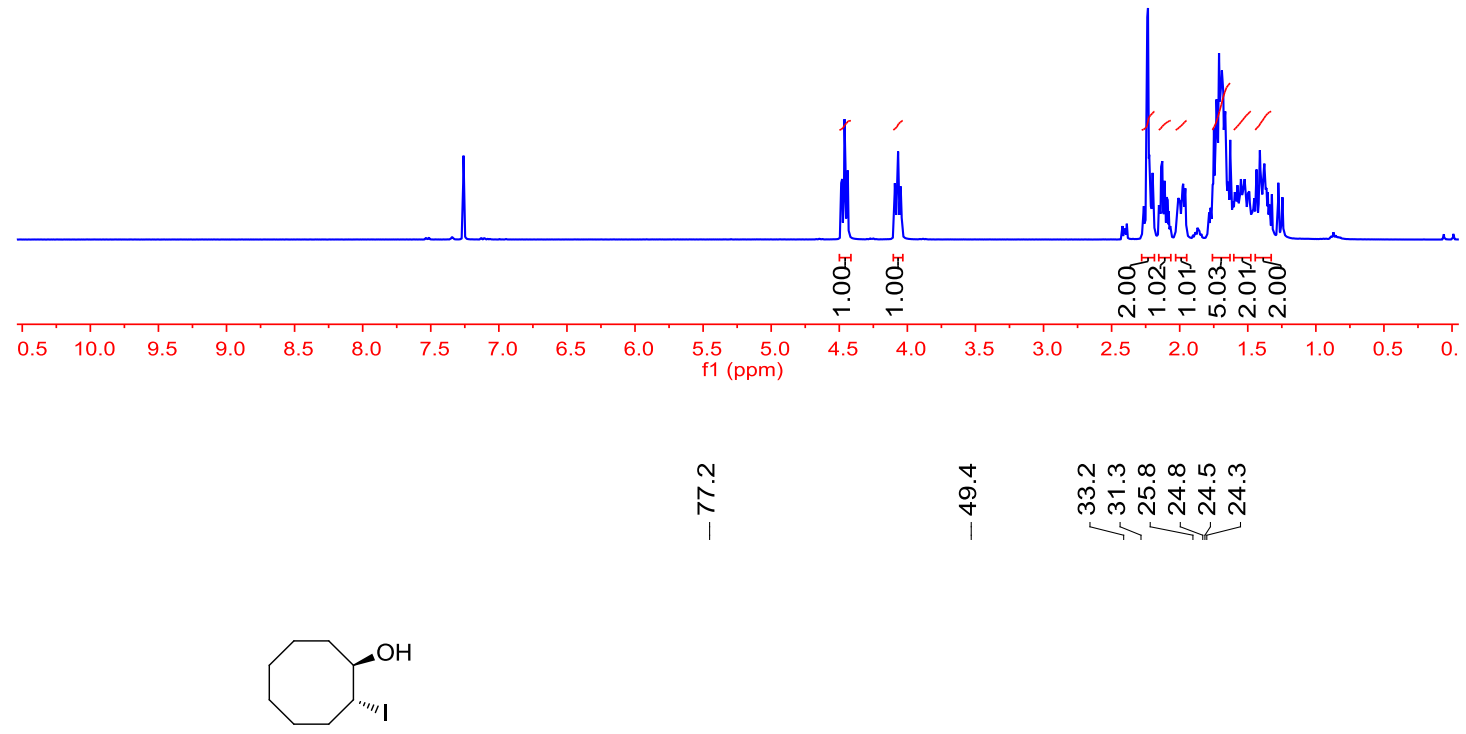

${ }^{13} \mathrm{C} \mathrm{NMR} \mathrm{(100} \mathrm{MHz,} \mathrm{CDCl}_{3}$ ) of $8 \mathbf{j}$

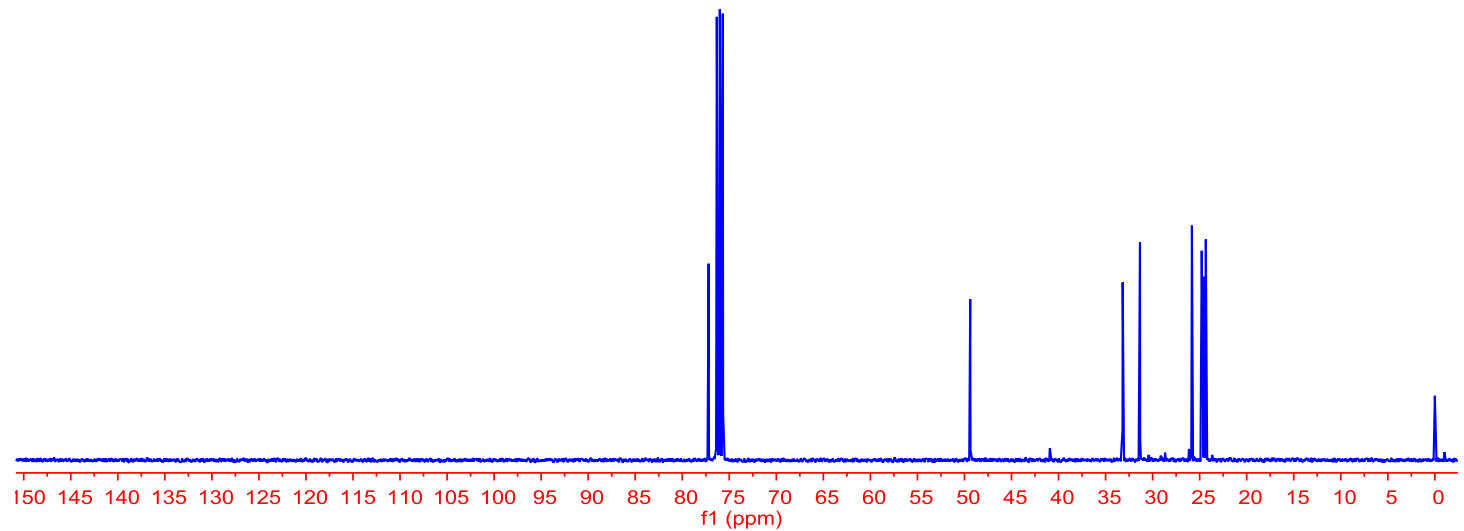




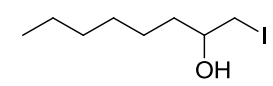

${ }^{1} \mathrm{H}$ NMR $\left(400 \mathrm{MHz}, \mathrm{CDCl}_{3}\right)$ of $\mathbf{8 k}$
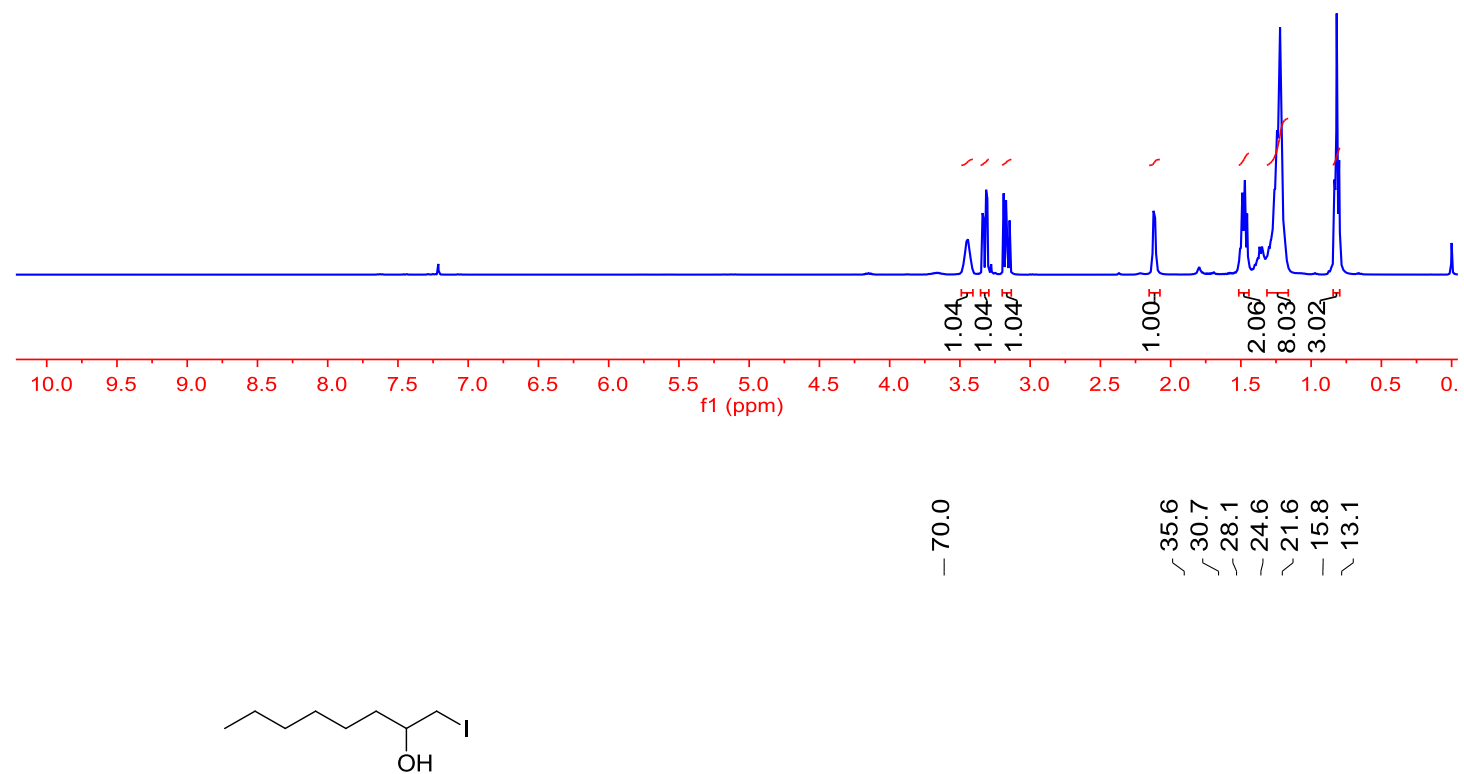

${ }^{13} \mathrm{C}$ NMR $\left(100 \mathrm{MHz}, \mathrm{CDCl}_{3}\right)$ of $\mathbf{8 k}$

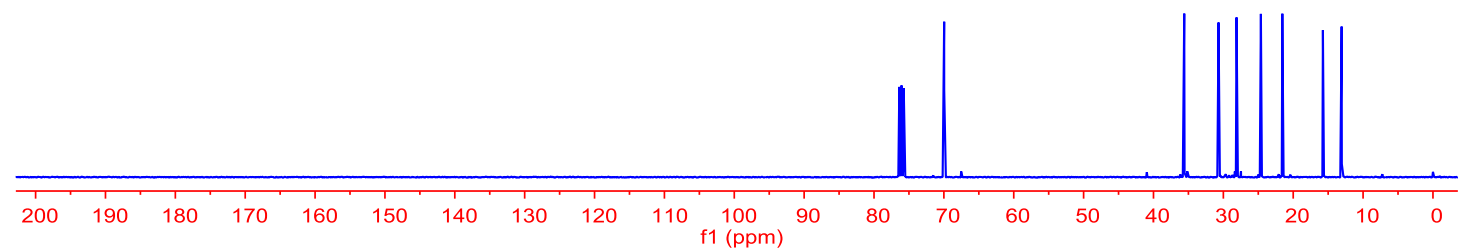




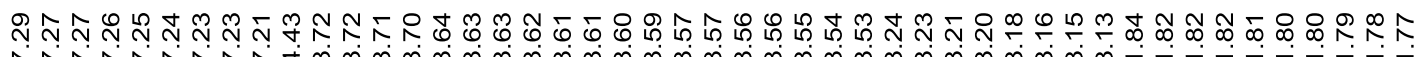

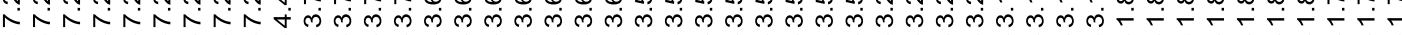<smiles>OC(CI)CCOCc1ccccc1</smiles>

${ }^{1} \mathrm{H}$ NMR $\left(400 \mathrm{MHz}, \mathrm{CDCl}_{3}\right)$ of 81

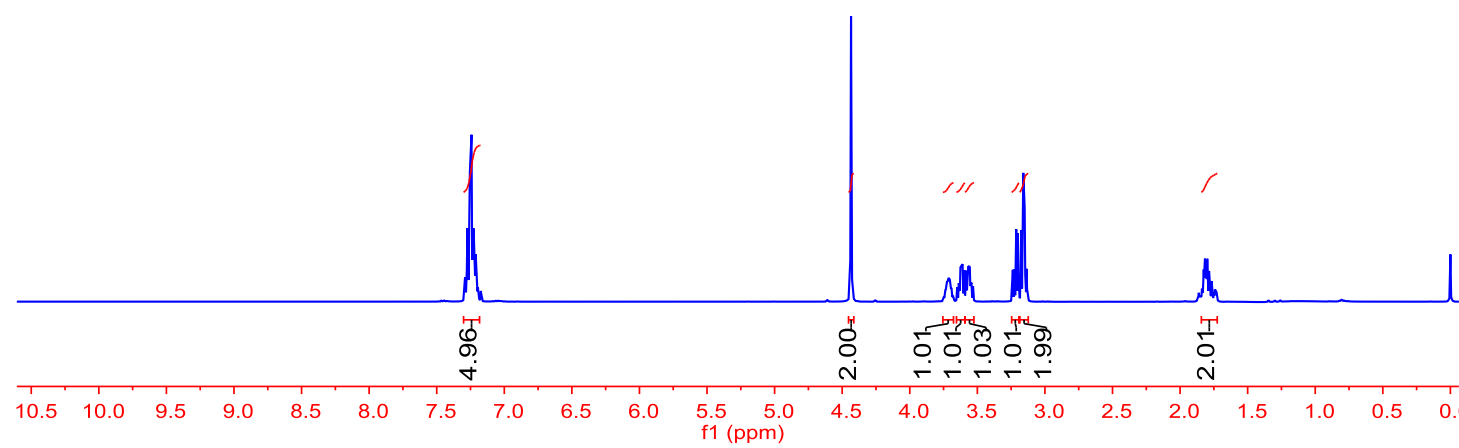

tis

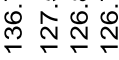

กิ

$\stackrel{\infty}{m} \quad \stackrel{m}{\dot{m}}$

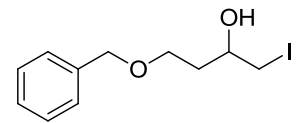

${ }^{1} \mathrm{H}$ NMR $\left(400 \mathrm{MHz}, \mathrm{CDCl}_{3}\right)$ of $\mathbf{8 I}$

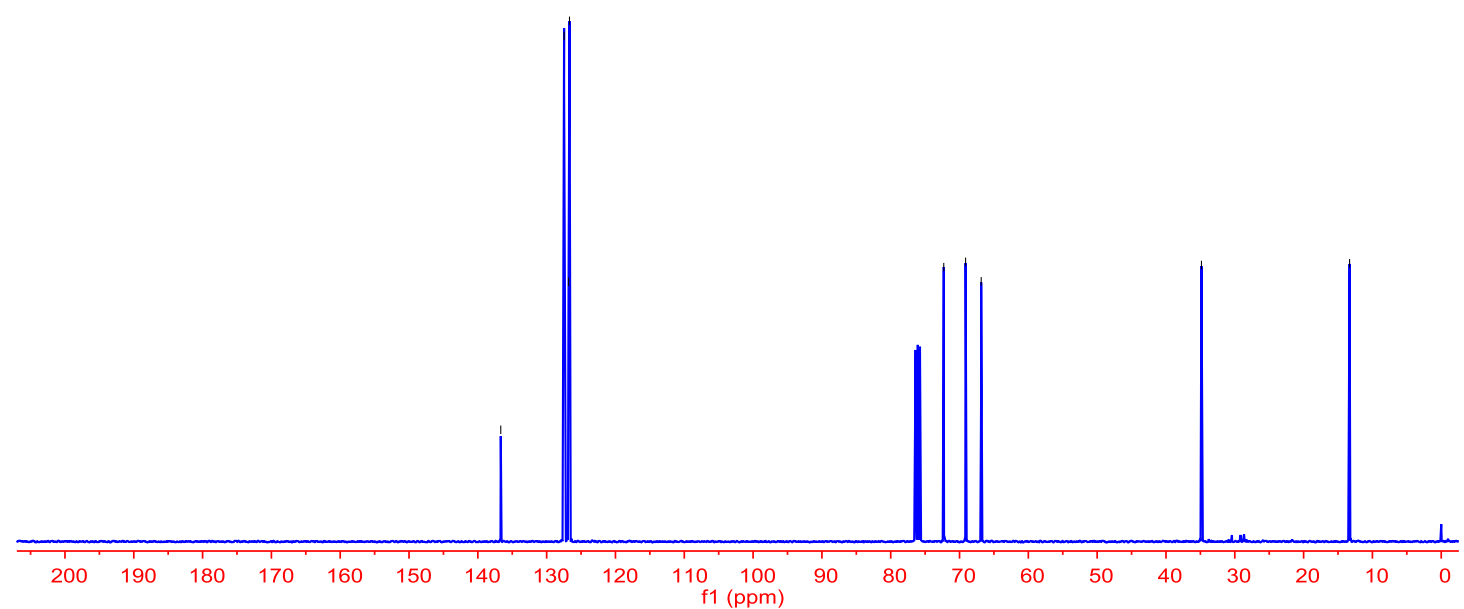




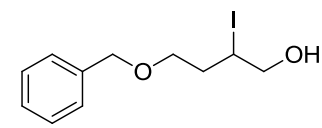

${ }^{1} \mathrm{H}$ NMR $\left(400 \mathrm{MHz}, \mathrm{CDCl}_{3}\right)$ of 8I'

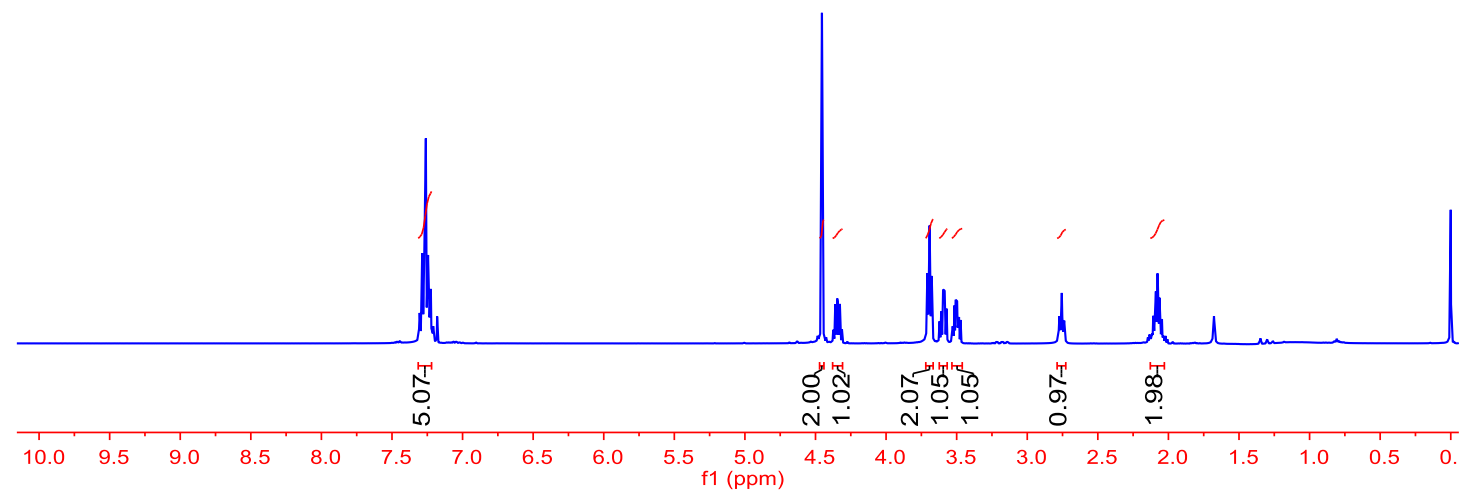

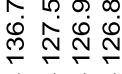
mị t.
N
$\ddot{0}+\dot{0}$
$\backslash>$
ले ले



${ }^{13} \mathrm{C} \mathrm{NMR}\left(100 \mathrm{MHz}, \mathrm{CDCl}_{3}\right)$ of $\mathbf{8} \mathbf{l}^{\prime}$

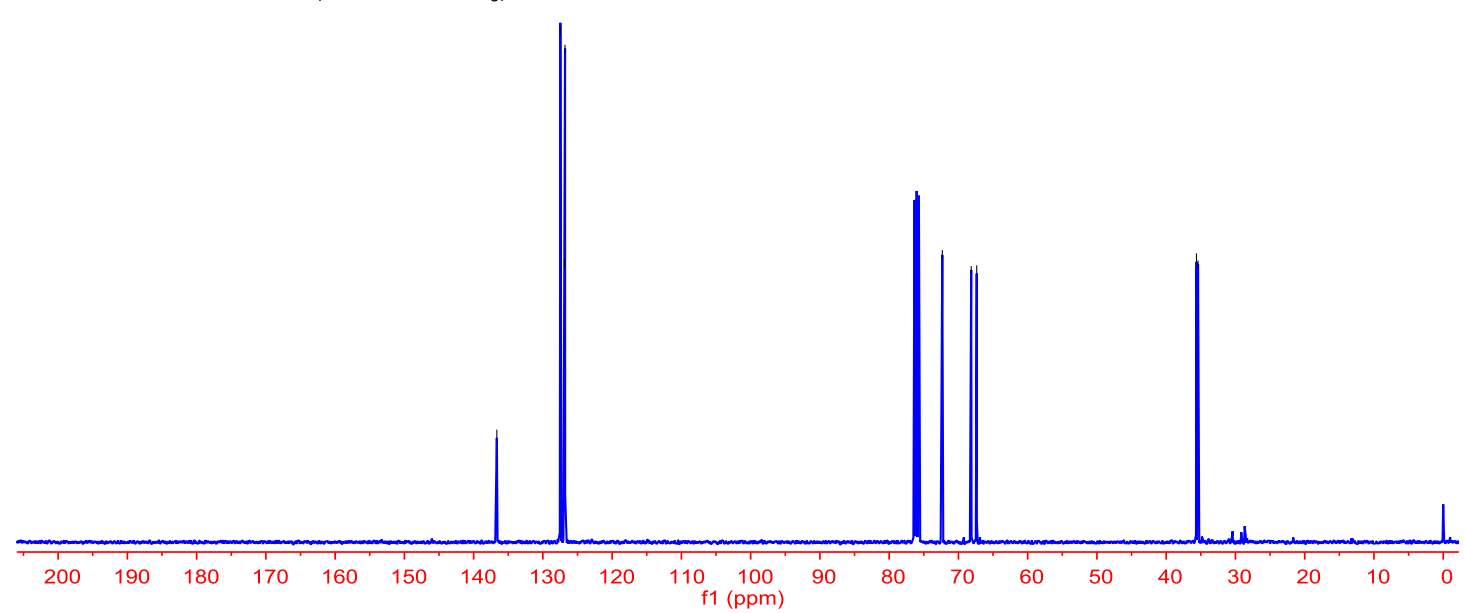




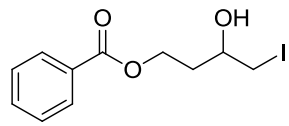

${ }^{1} \mathrm{H} \mathrm{NMR}\left(400 \mathrm{MHz}, \mathrm{CDCl}_{3}\right)$ of $8 \mathrm{~m}$

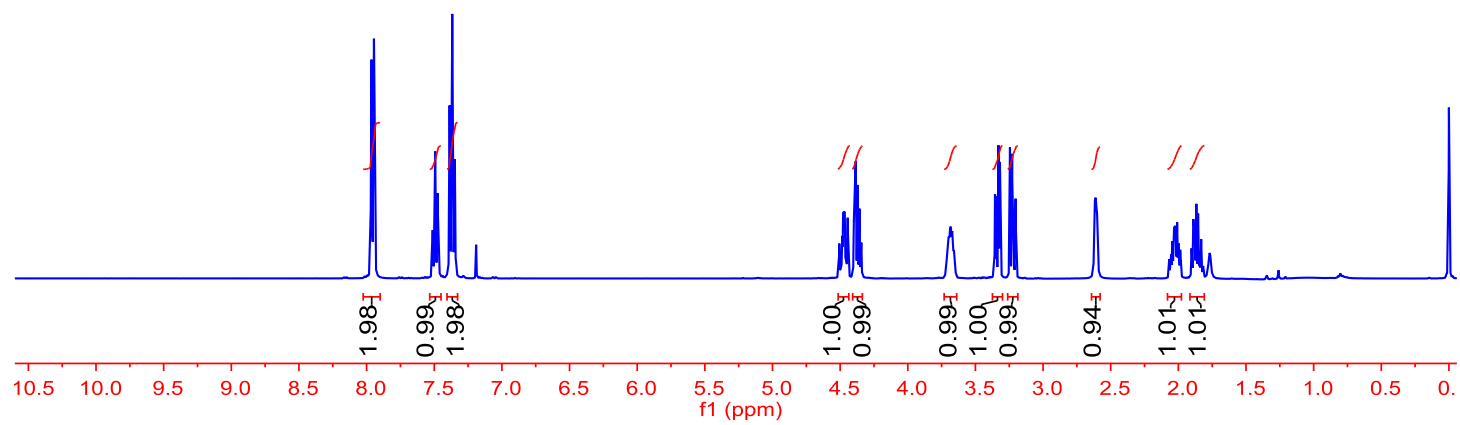

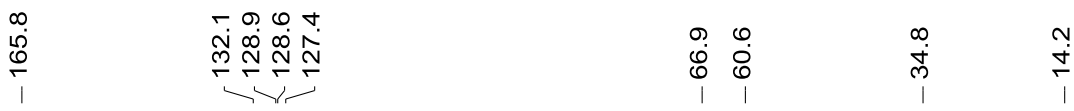

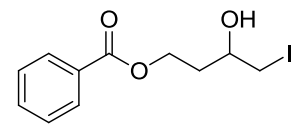

${ }^{1} \mathrm{H} \mathrm{NMR}\left(400 \mathrm{MHz}, \mathrm{CDCl}_{3}\right)$ of $8 \mathrm{~m}$

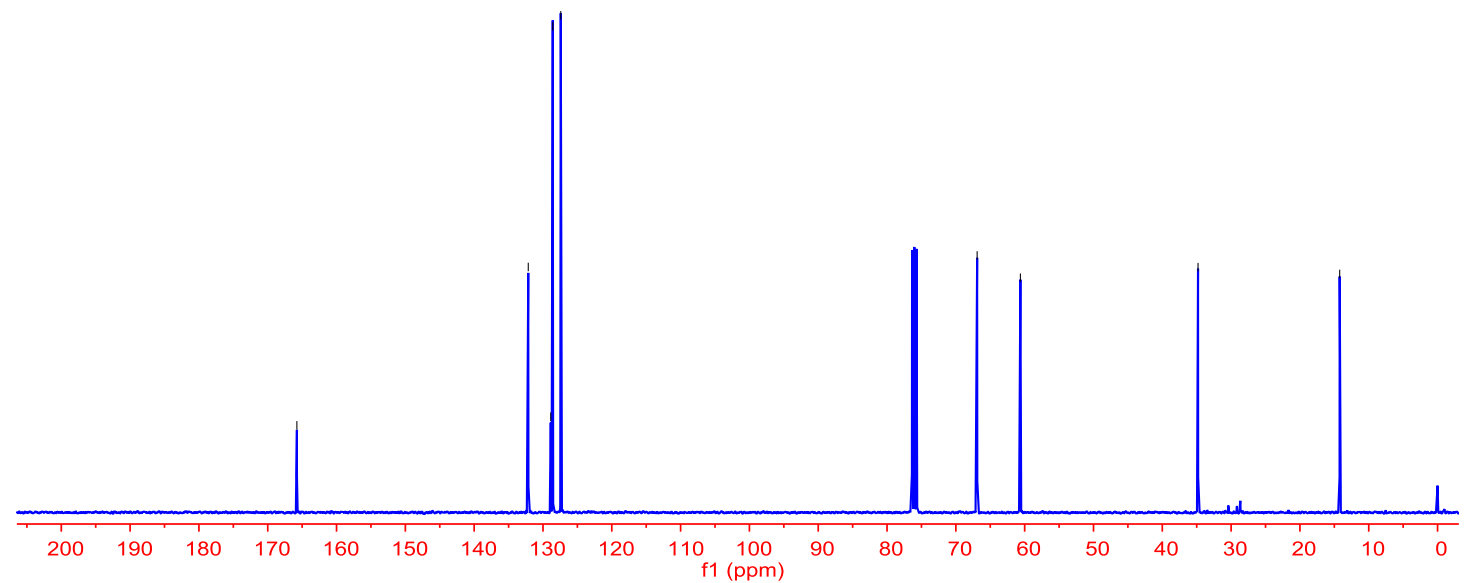




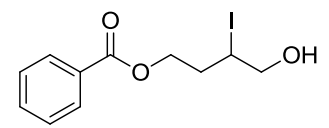

${ }^{1} \mathrm{H} \mathrm{NMR}\left(400 \mathrm{MHz}, \mathrm{CDCl}_{3}\right)$ of $8 \mathrm{~m}$ '
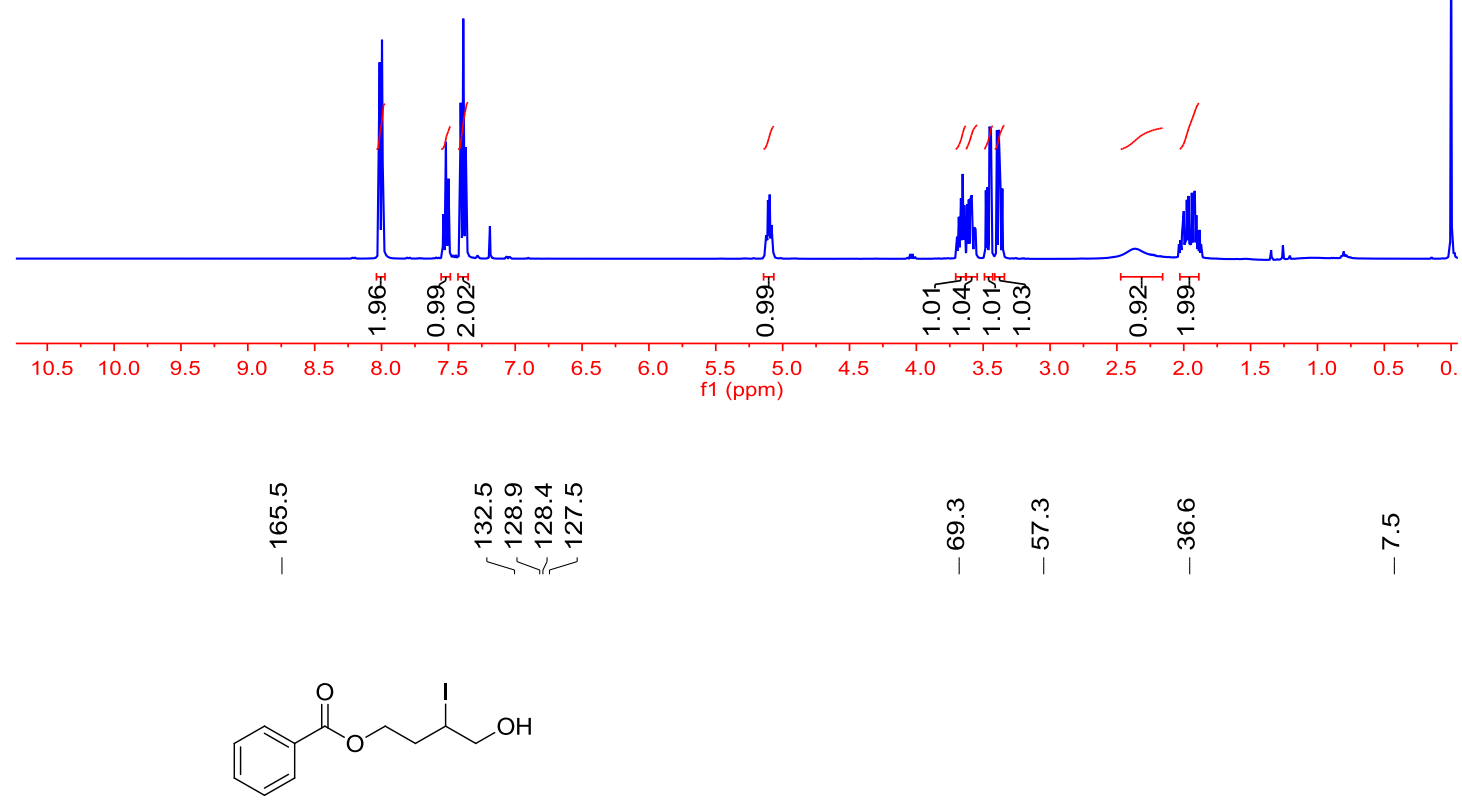

${ }^{13} \mathrm{C}$ NMR $\left(100 \mathrm{MHz}, \mathrm{CDCl}_{3}\right)$ of $\mathbf{8 m}$ '

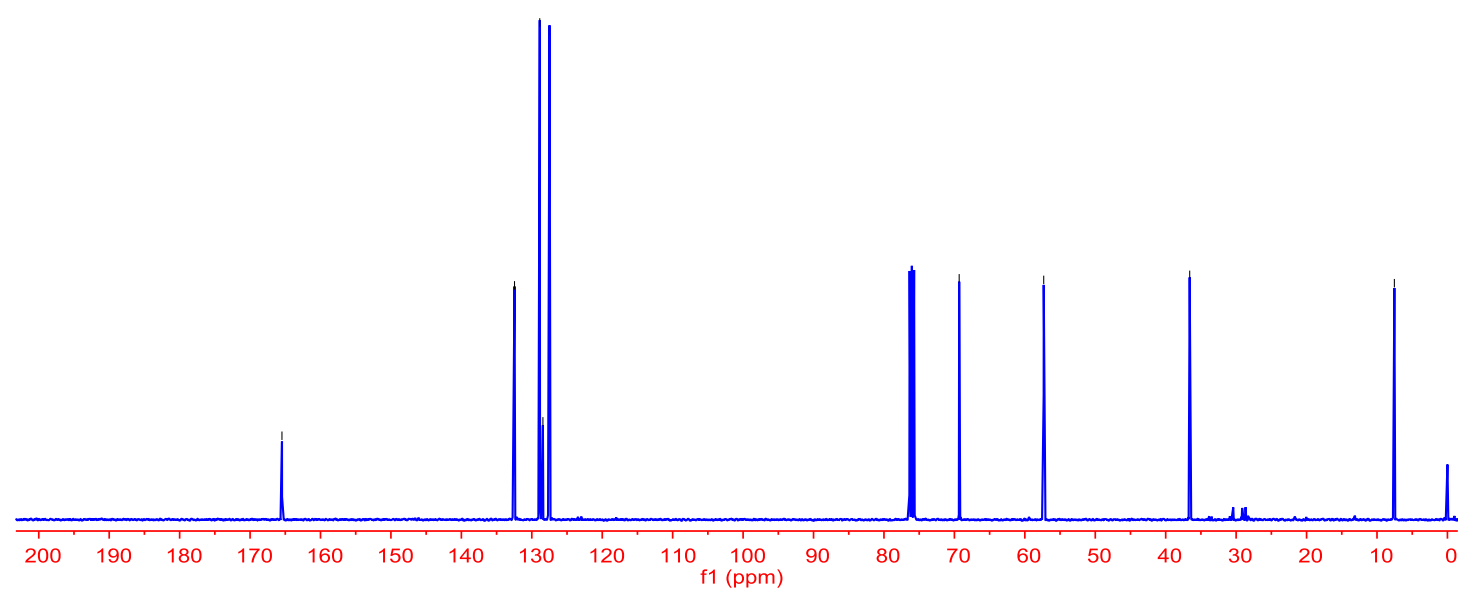



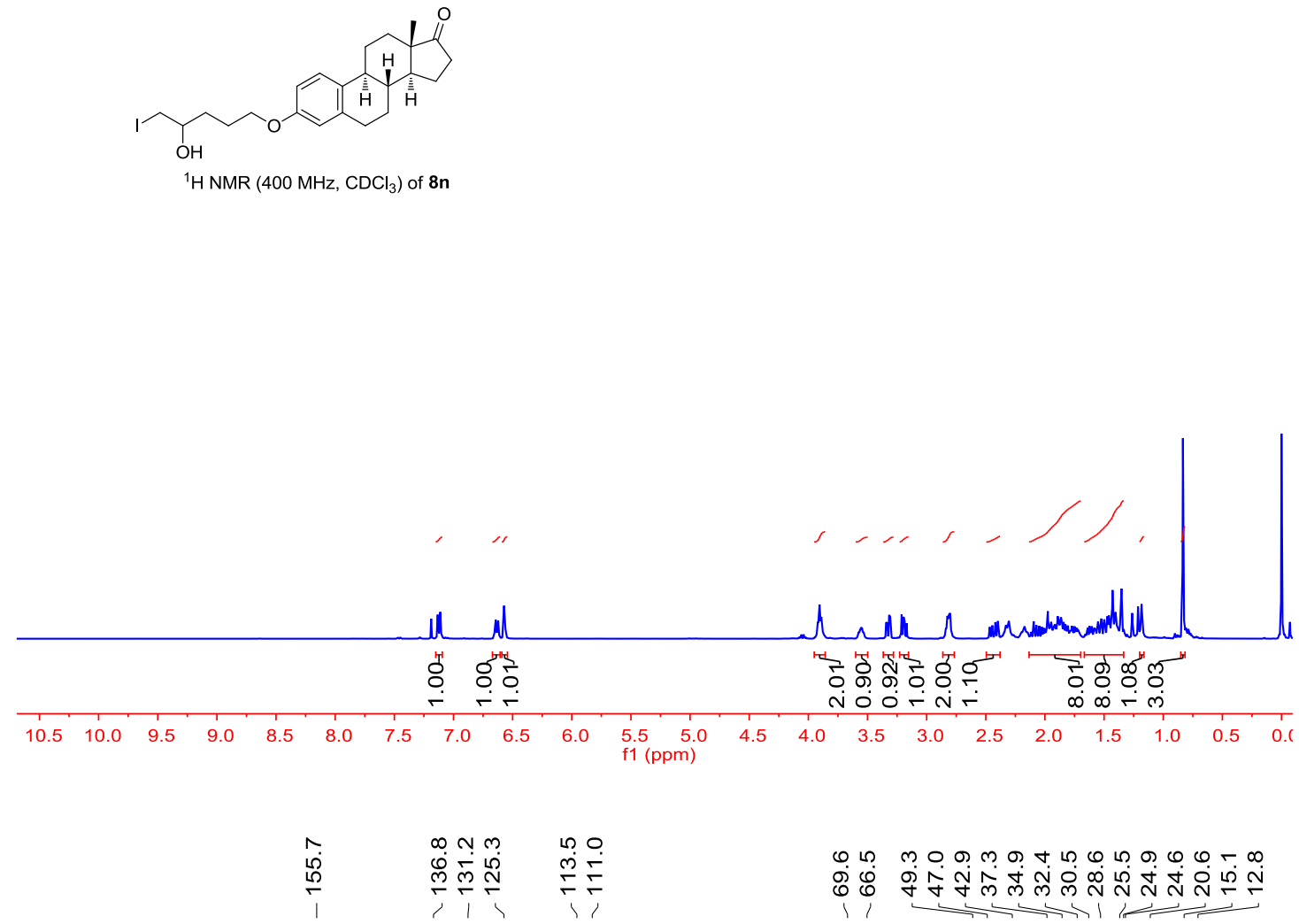

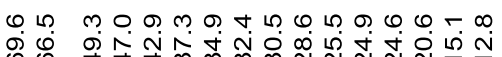

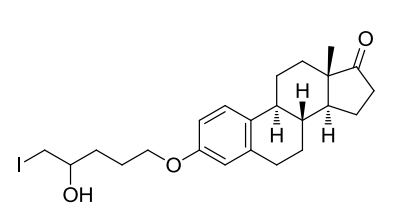

${ }^{13} \mathrm{C} \mathrm{NMR}\left(100 \mathrm{MHz}, \mathrm{CDCl}_{3}\right)$ of $\mathbf{8 n}$

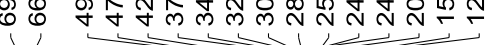

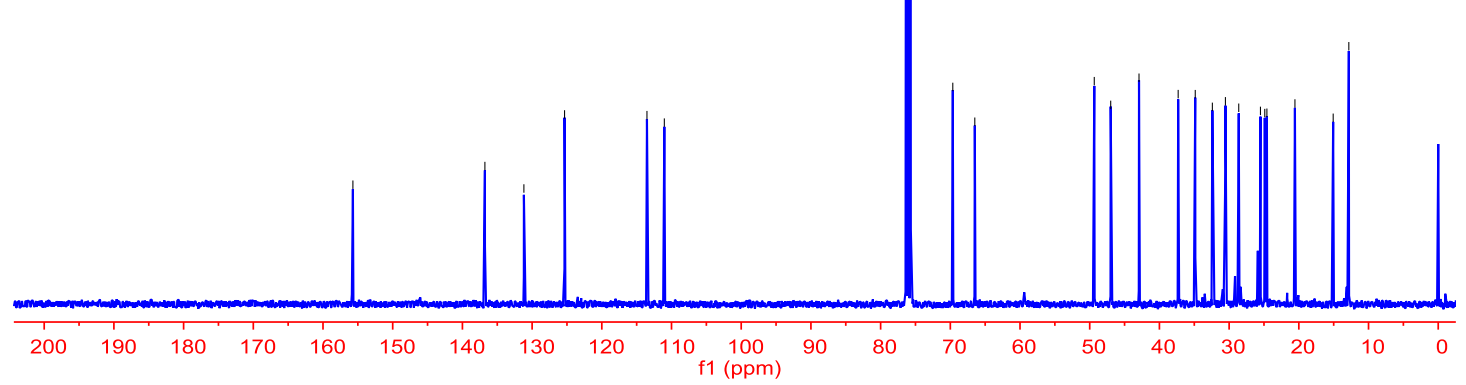




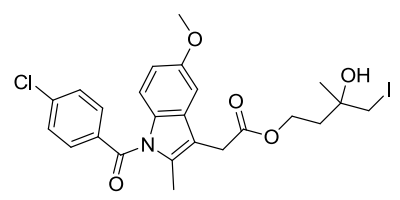

${ }^{1} \mathrm{H} \mathrm{NMR}\left(400 \mathrm{MHz}, \mathrm{CDCl}_{3}\right)$ of 80

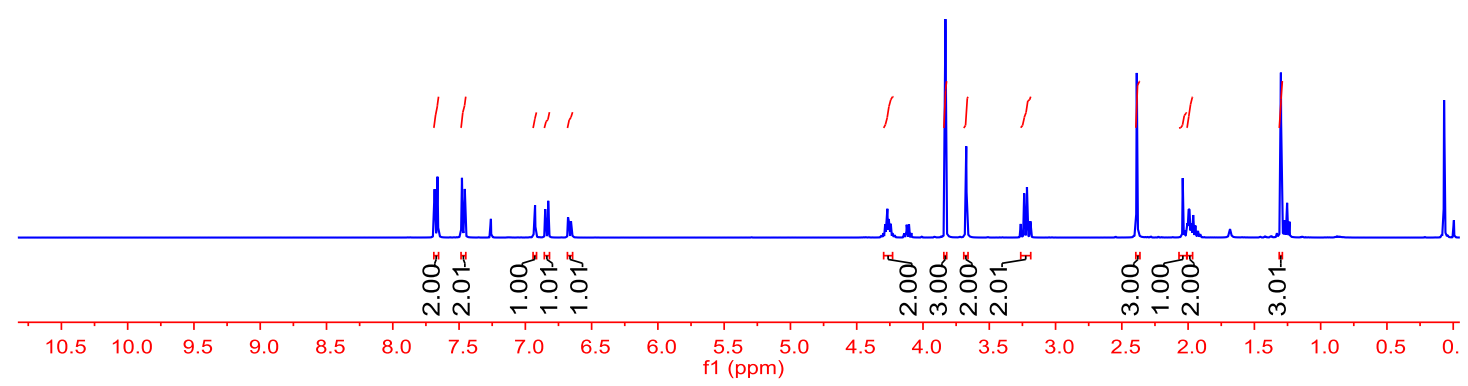

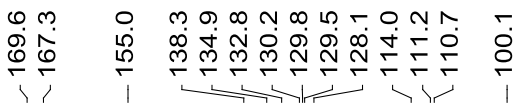

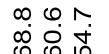

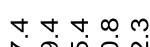

近

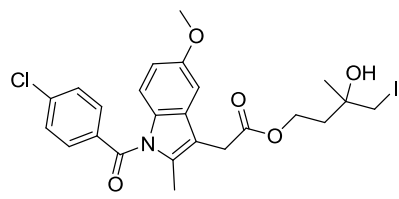

${ }^{13} \mathrm{C} \mathrm{NMR}\left(100 \mathrm{MHz}, \mathrm{CDCl}_{3}\right)$ of 80

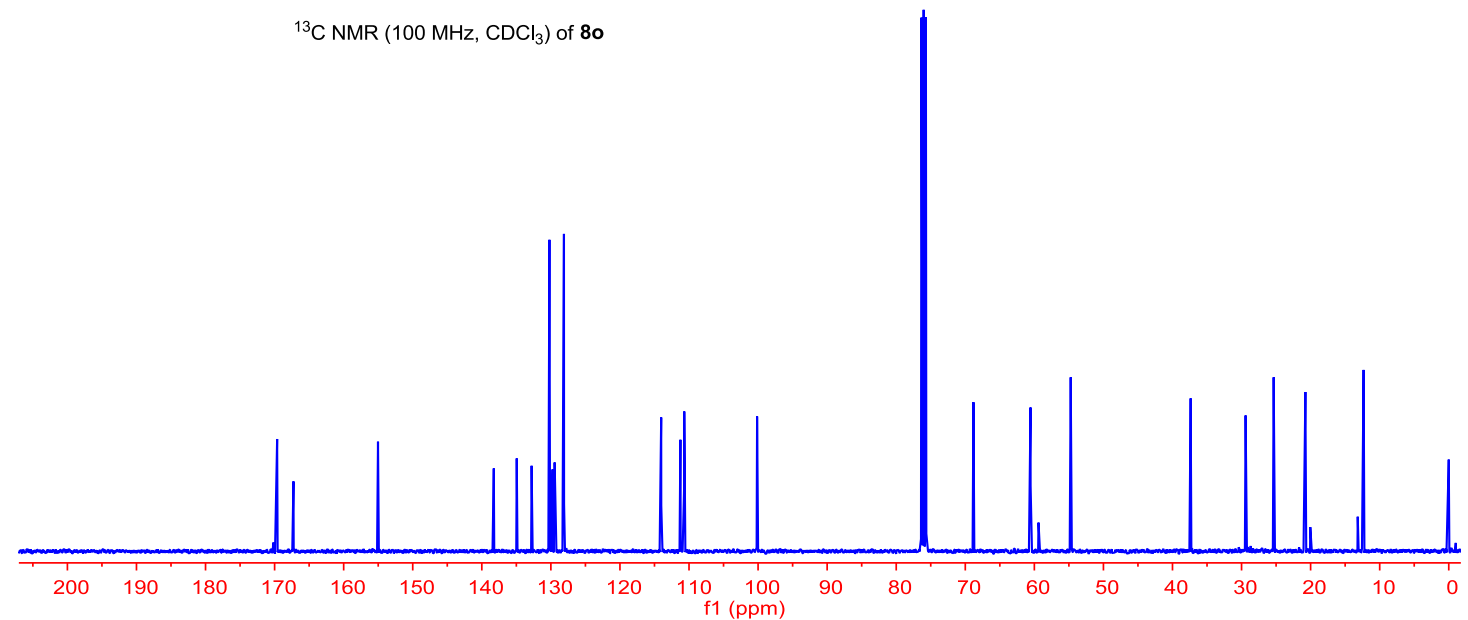




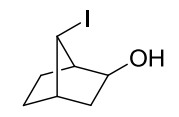

${ }^{1} \mathrm{H}$ NMR $\left(400 \mathrm{MHz}, \mathrm{CDCl}_{3}\right)$ of $\mathbf{8 p}$

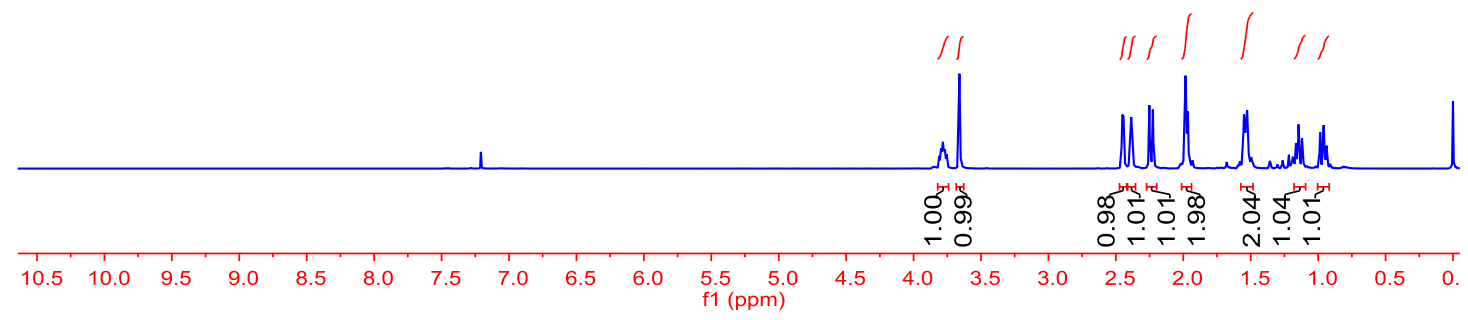

की

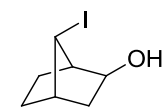

${ }^{13} \mathrm{C}$ NMR (100 MHz, $\mathrm{CDCl}_{3}$ ) of $\mathbf{8 p}$

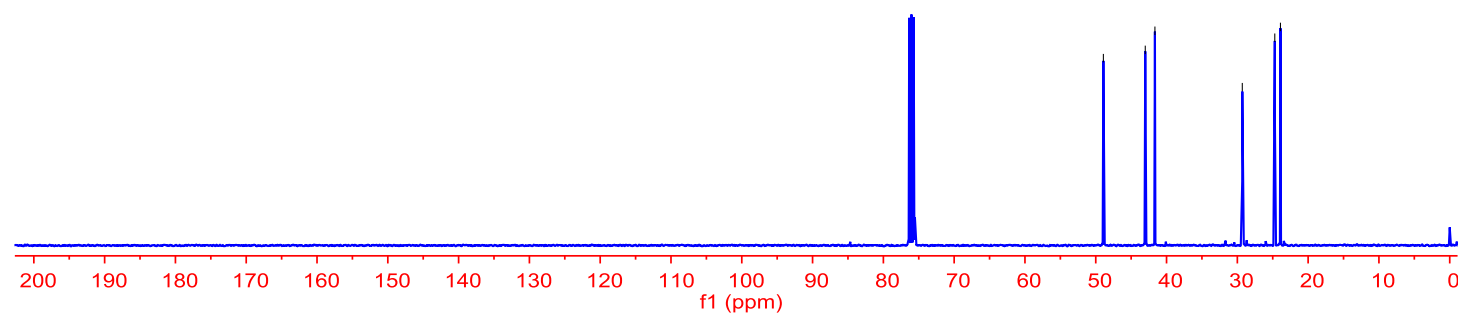




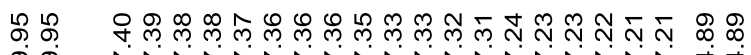

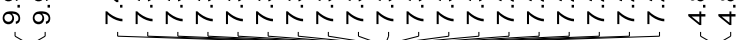

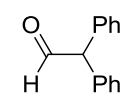

${ }^{1} \mathrm{H}$ NMR $\left(400 \mathrm{MHz}, \mathrm{CDCl}_{3}\right)$ of $\mathbf{8 q}$

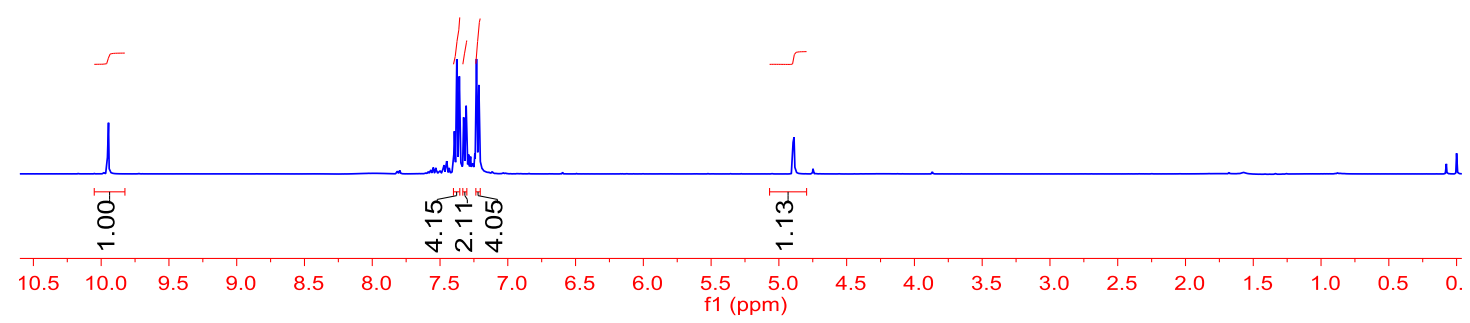

0
$\infty$
$\infty$
$\stackrel{1}{1}$

m No:

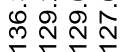

¿

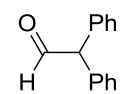

${ }^{13} \mathrm{C} \mathrm{NMR}\left(100 \mathrm{MHz}, \mathrm{CDCl}_{3}\right)$ of $\mathbf{8 q}$

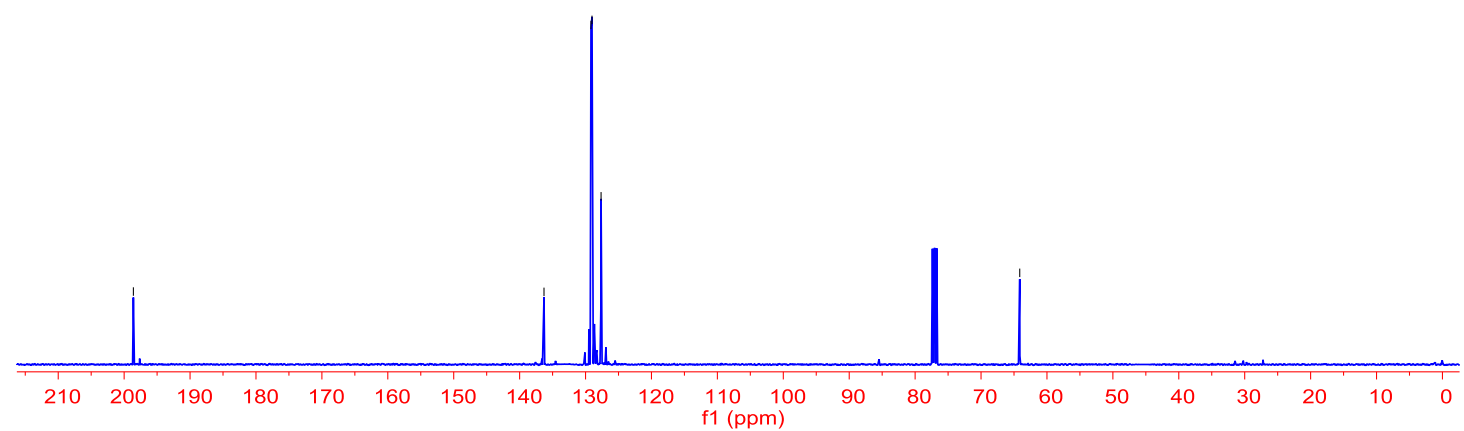




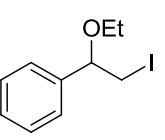

${ }^{1} \mathrm{H}$ NMR $\left(400 \mathrm{MHz}, \mathrm{CDCl}_{3}\right)$ of $9 \mathrm{a}$

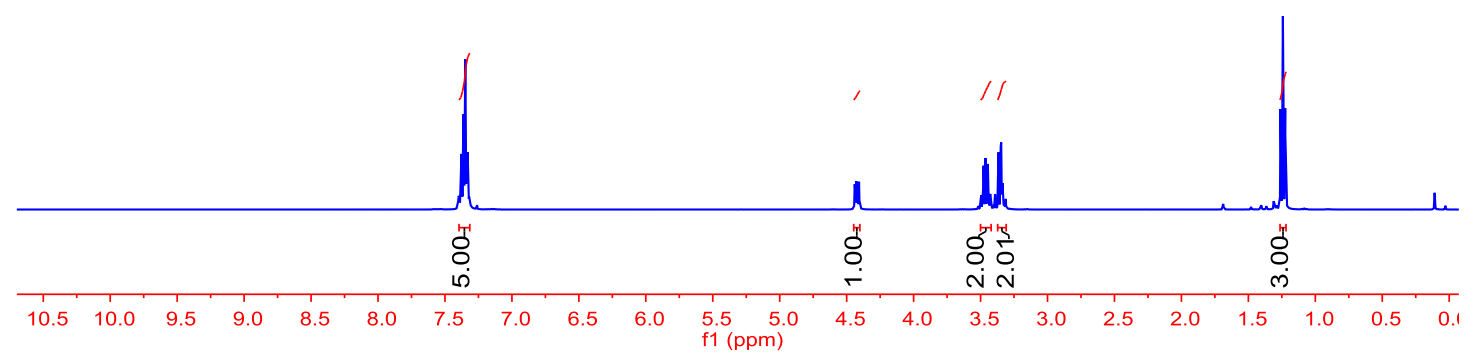

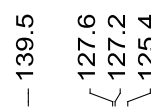

$\begin{array}{ll}\infty & 0 \\ 0 & \dot{0} \\ 1 & 0\end{array}$

$\div \%$

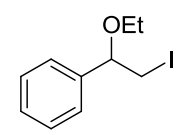

${ }^{13} \mathrm{C}$ NMR $\left(100 \mathrm{MHz}, \mathrm{CDCl}_{3}\right)$ of $\mathbf{9 a}$

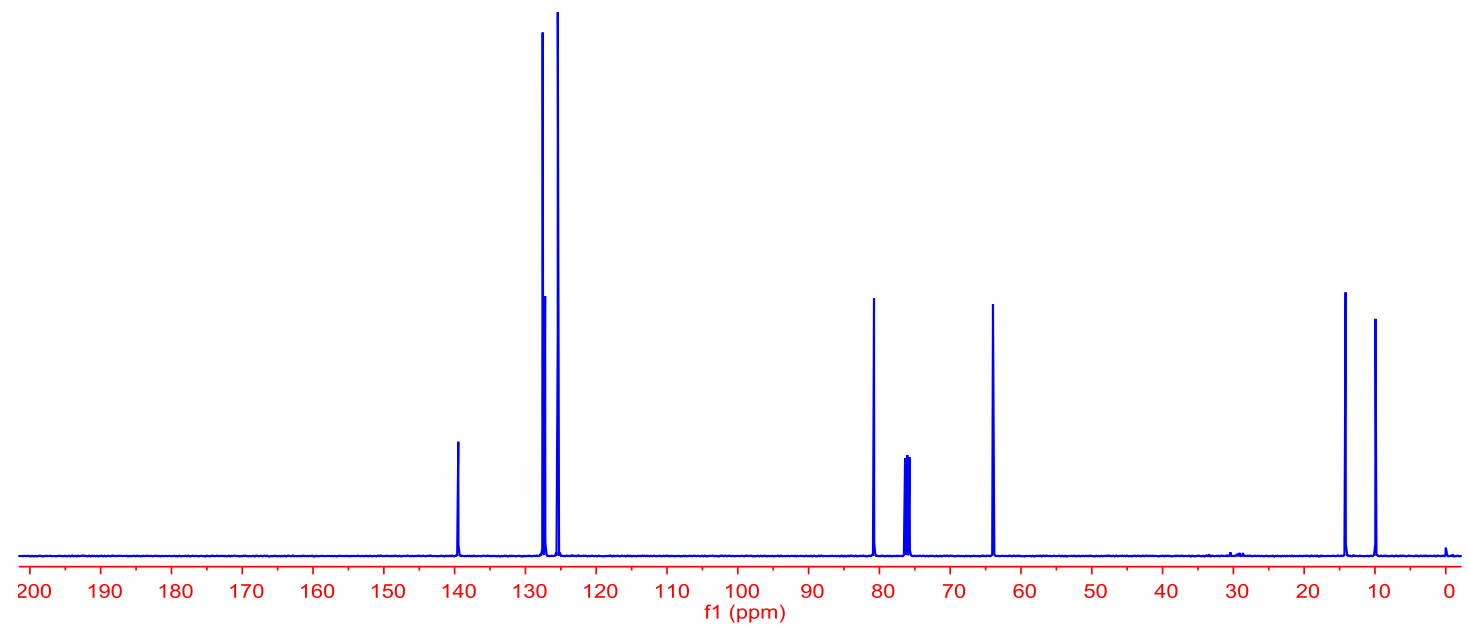


NMR (400 $\mathrm{MHz}, \mathrm{CDCl}_{3}$ ) of $\mathbf{9 b}$

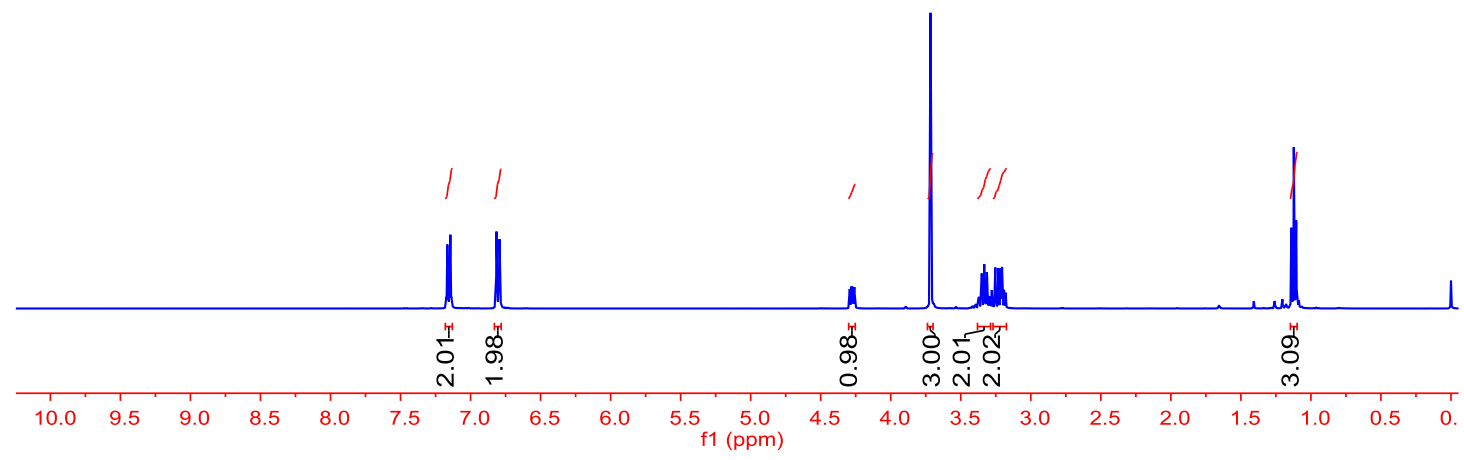

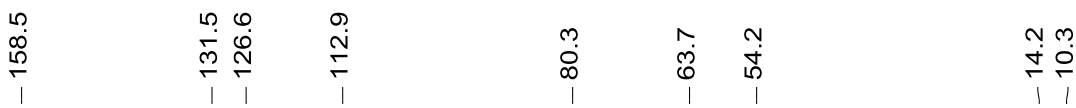

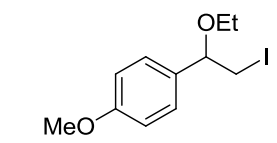

${ }^{13} \mathrm{C}$ NMR $\left(100 \mathrm{MHz}, \mathrm{CDCl}_{3}\right)$ of $\mathbf{9 b}$

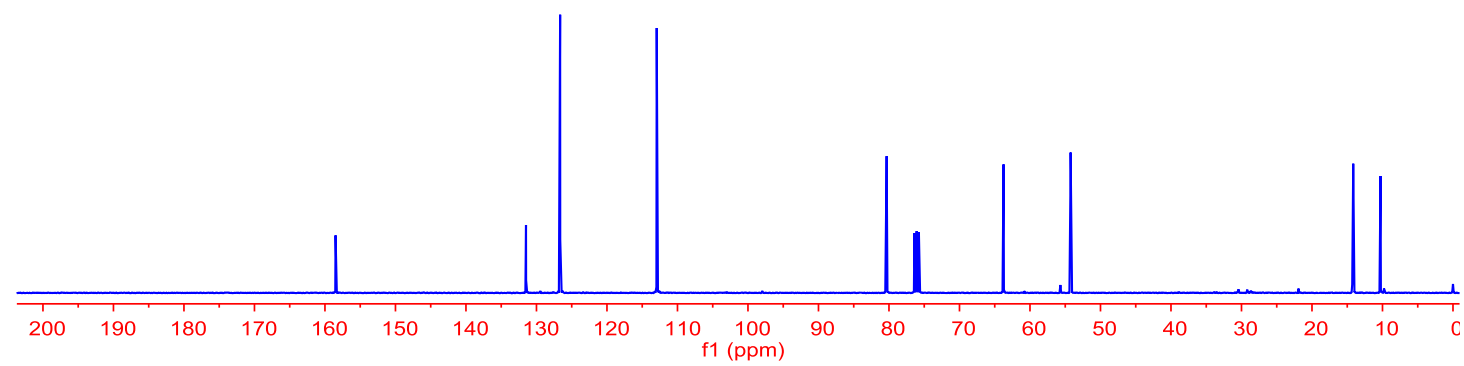


กิ

గ

NNN

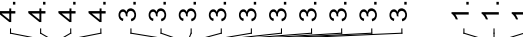

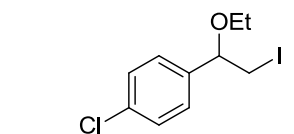

${ }^{1} \mathrm{H} \mathrm{NMR}\left(400 \mathrm{MHz}, \mathrm{CDCl}_{3}\right)$ of $9 \mathrm{c}$

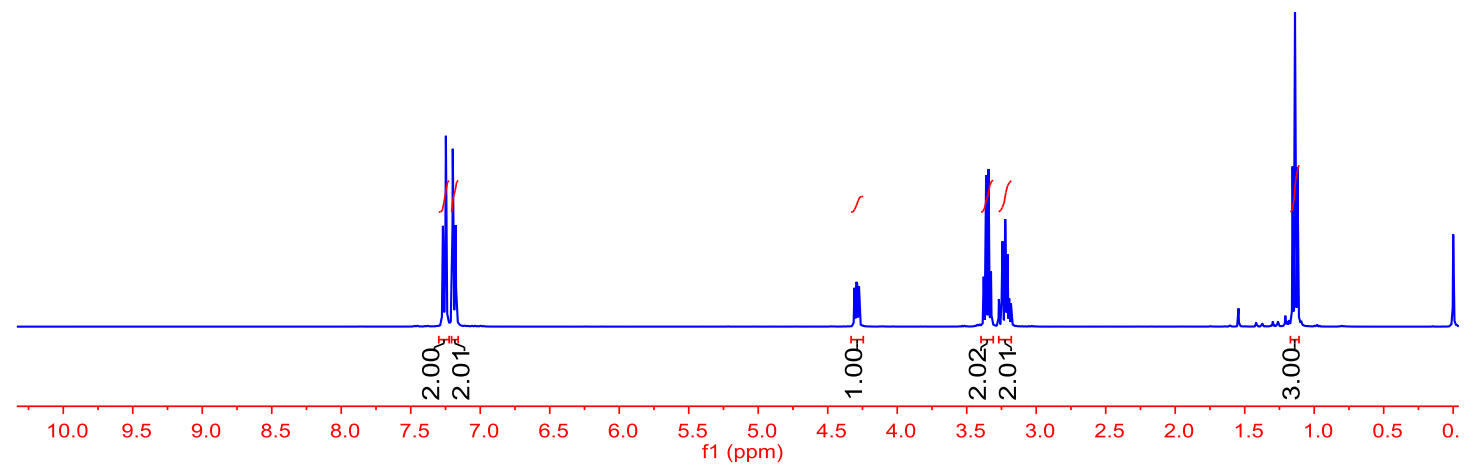

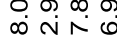

$\stackrel{m}{\sim} \stackrel{\sim}{\sim} \stackrel{\sim}{\sim} \stackrel{0}{N}$

$\begin{array}{ll}\circ & \dot{0} \\ 0 & 0 \\ 1 & 1\end{array}$

$\stackrel{+}{+}$

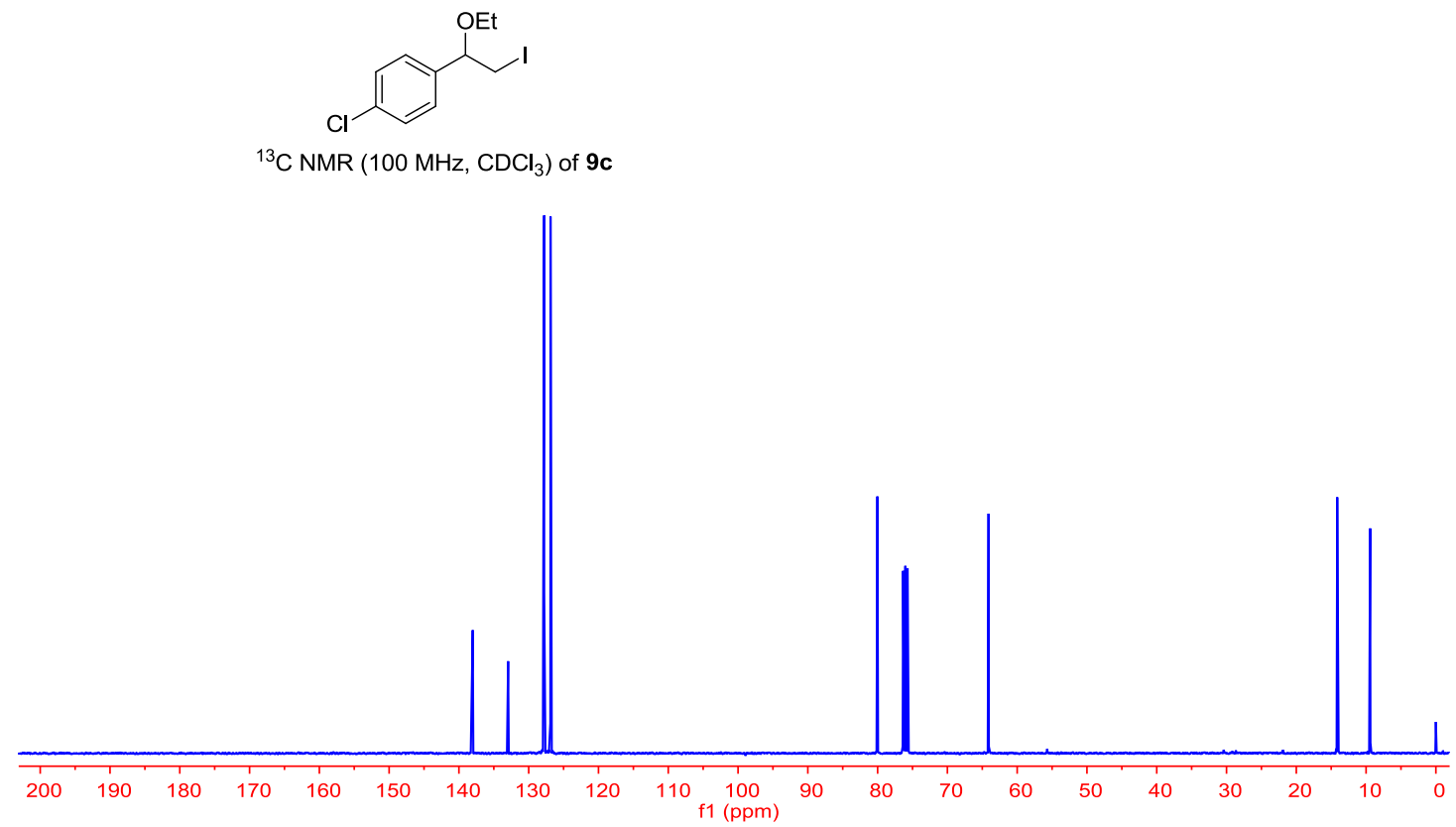




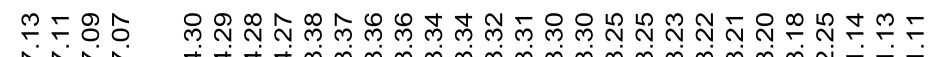

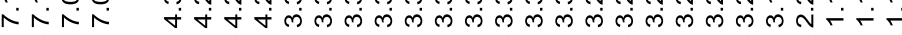

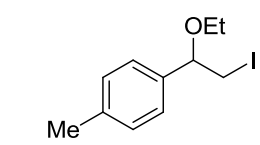

${ }^{1} \mathrm{H} \mathrm{NMR}\left(400 \mathrm{MHz}, \mathrm{CDCl}_{3}\right)$ of $\mathbf{9 d}$

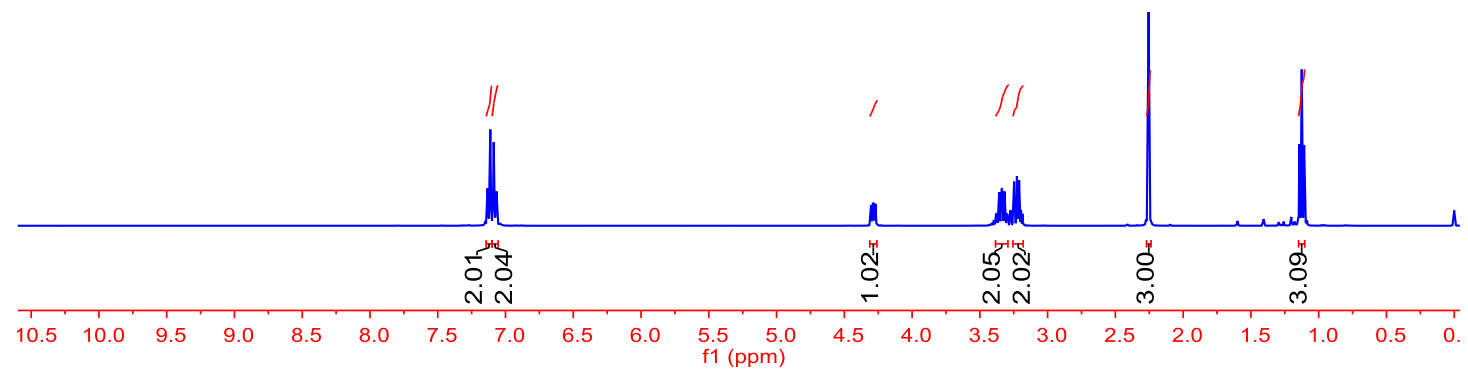

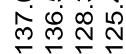

$\begin{array}{cc}0 & \infty \\ 0 & 0 \\ 0 & 0 \\ 1 & 0\end{array}$

ํํำ

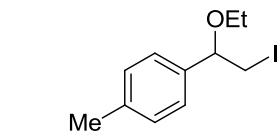

${ }^{13} \mathrm{C} \mathrm{NMR} \mathrm{(100} \mathrm{MHz,} \mathrm{CDCl}_{3}$ ) of $9 d$

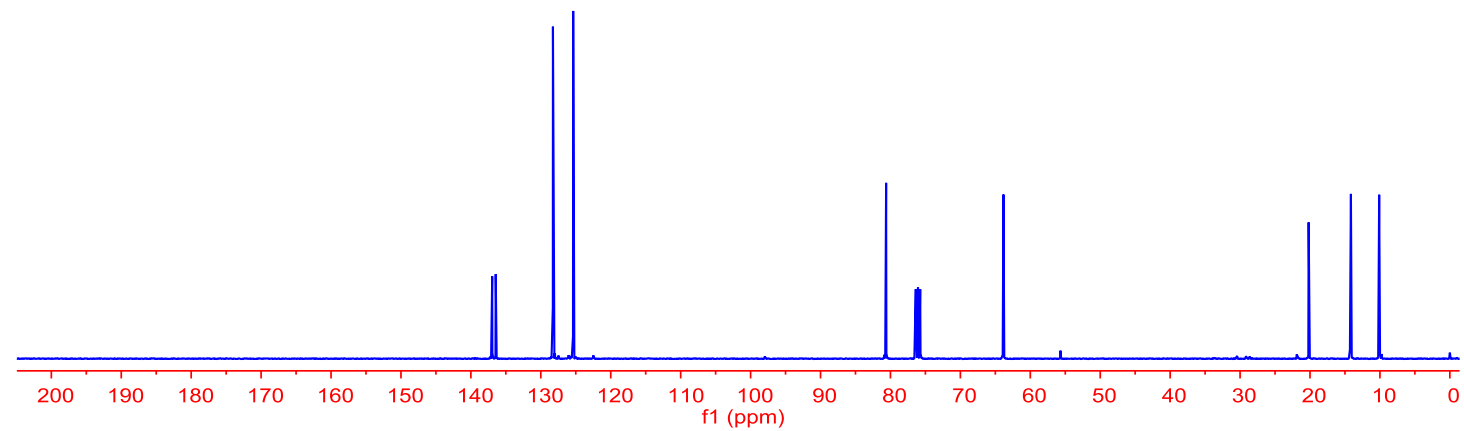




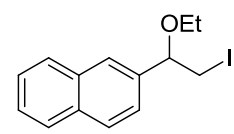

${ }^{1} \mathrm{H} \mathrm{NMR}\left(400 \mathrm{MHz}, \mathrm{CDCl}_{3}\right)$ of $9 e$

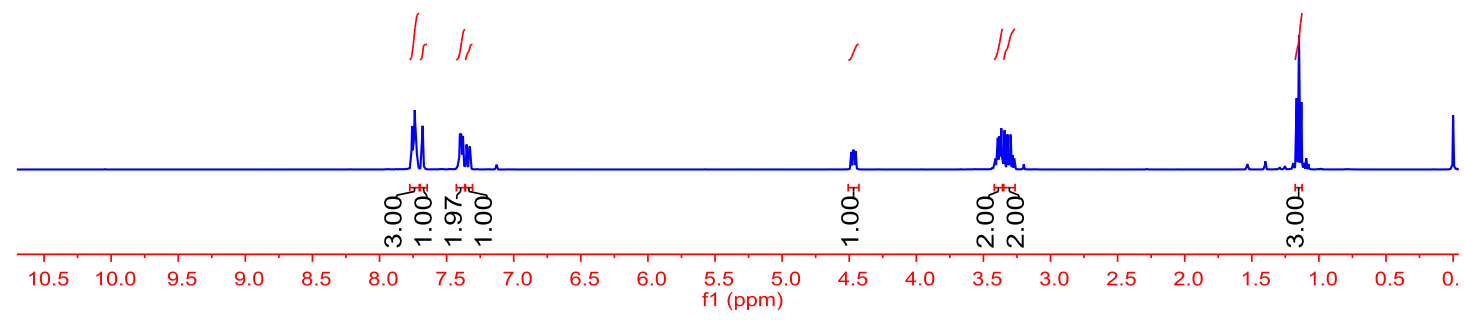

$\infty m-\infty, \pi)$

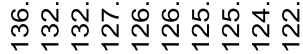

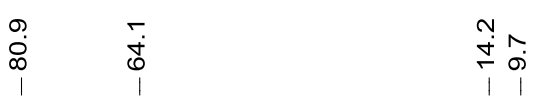

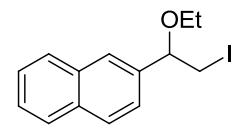

${ }^{13} \mathrm{C} \mathrm{NMR}\left(100 \mathrm{MHz}, \mathrm{CDCl}_{3}\right)$ of $9 \mathbf{e}$

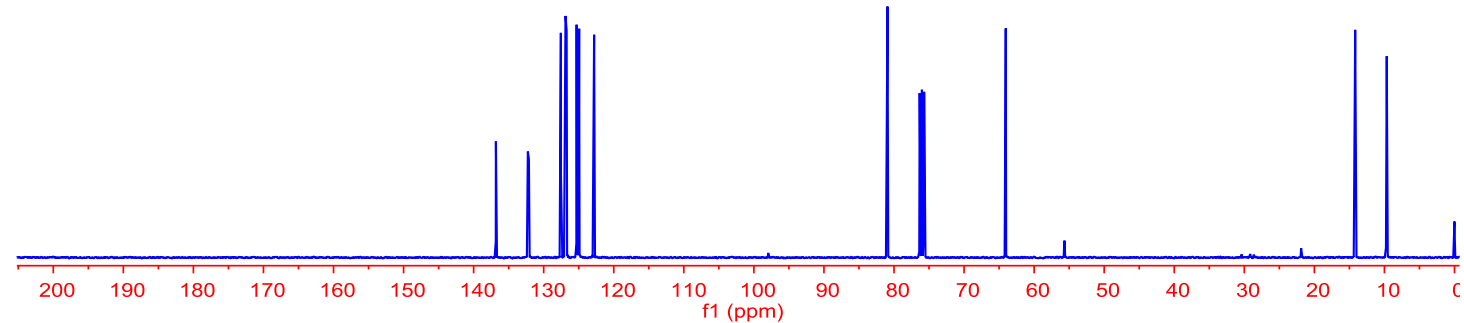




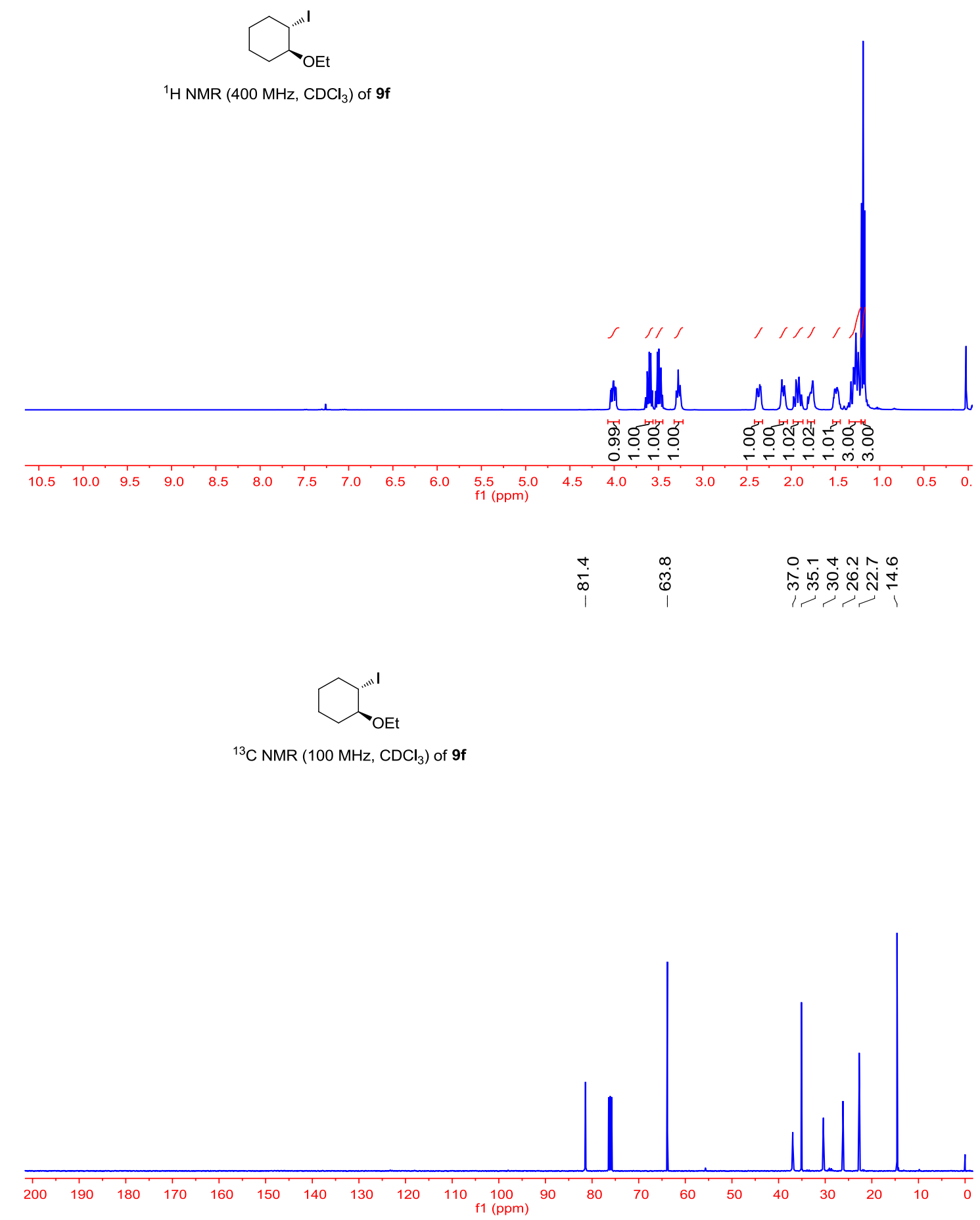




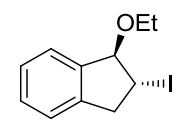

${ }^{1} \mathrm{H} \mathrm{NMR}\left(400 \mathrm{MHz}, \mathrm{CDCl}_{3}\right)$ of $\mathbf{9 g}$

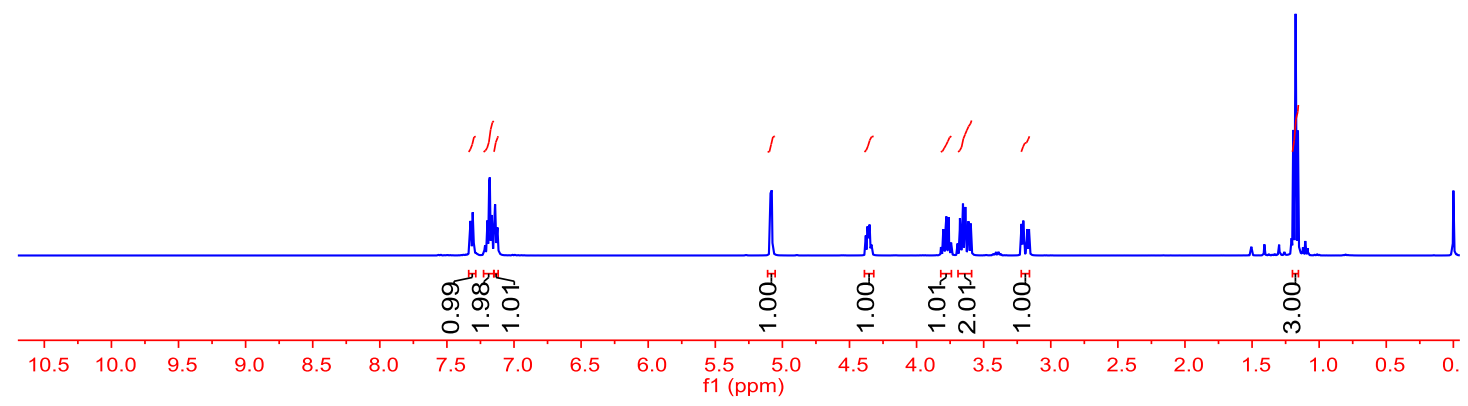

ก

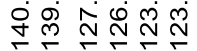

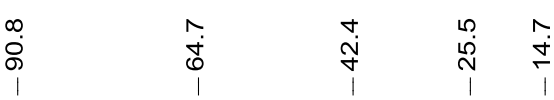

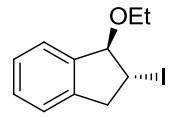

${ }^{13} \mathrm{C} \mathrm{NMR} \mathrm{(100} \mathrm{MHz,} \mathrm{CDCl}_{3}$ ) of $\mathbf{9 g}$

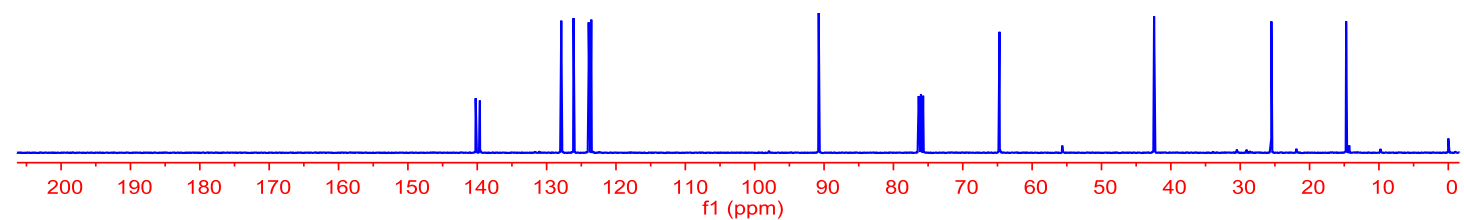




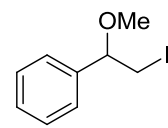

${ }^{1} \mathrm{H} \mathrm{NMR}\left(400 \mathrm{MHz}, \mathrm{CDCl}_{3}\right)$ of $\mathbf{9 h}$

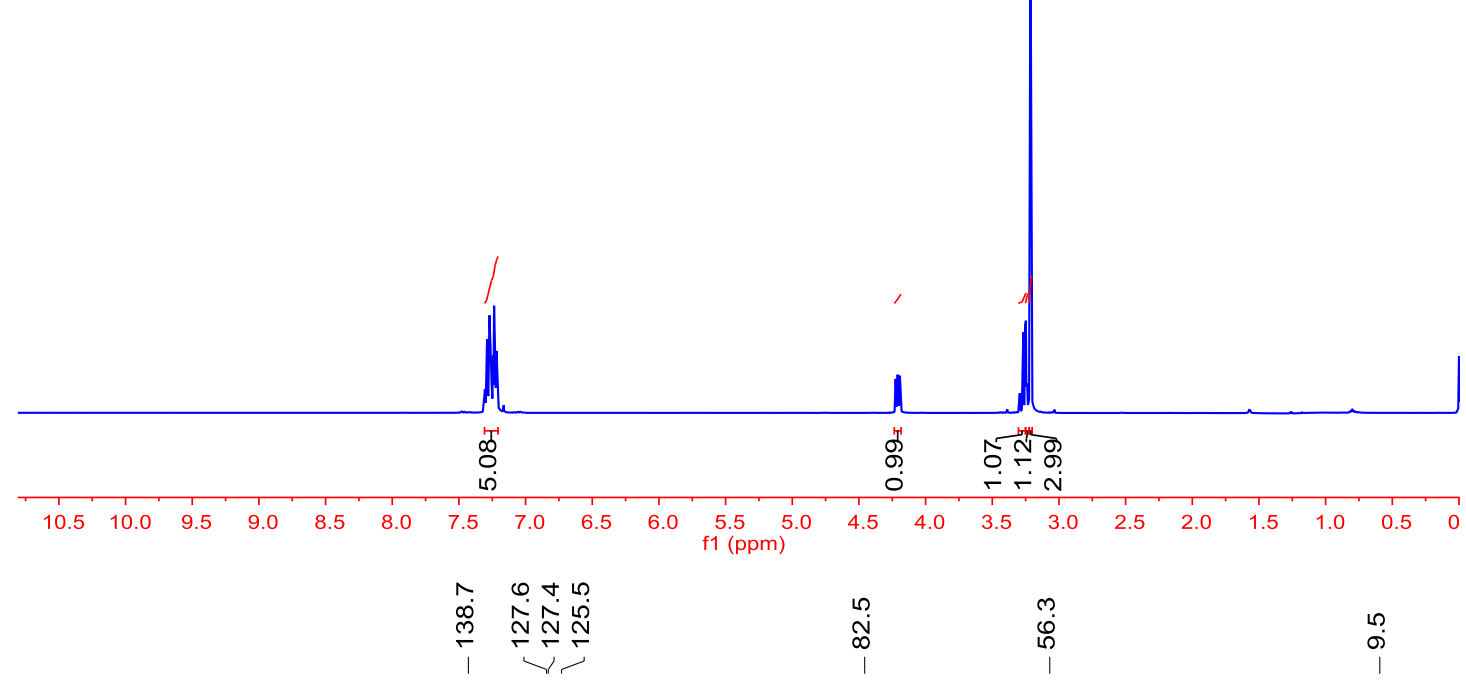

OMe

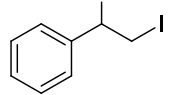

${ }^{13} \mathrm{C} \mathrm{NMR}\left(100 \mathrm{MHz}, \mathrm{CDCl}_{3}\right)$ of $\mathbf{9 h}$

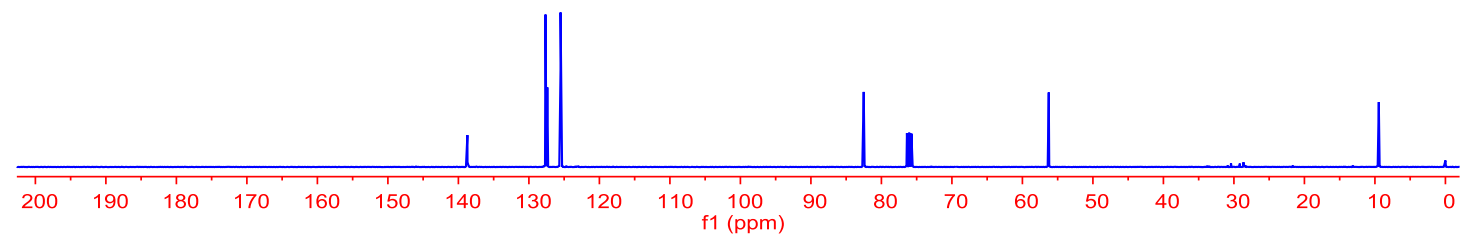




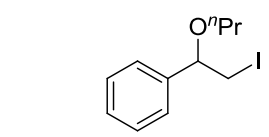

${ }^{1} \mathrm{H} \mathrm{NMR}\left(400 \mathrm{MHz}, \mathrm{CDCl}_{3}\right)$ of $\mathbf{9 i}$

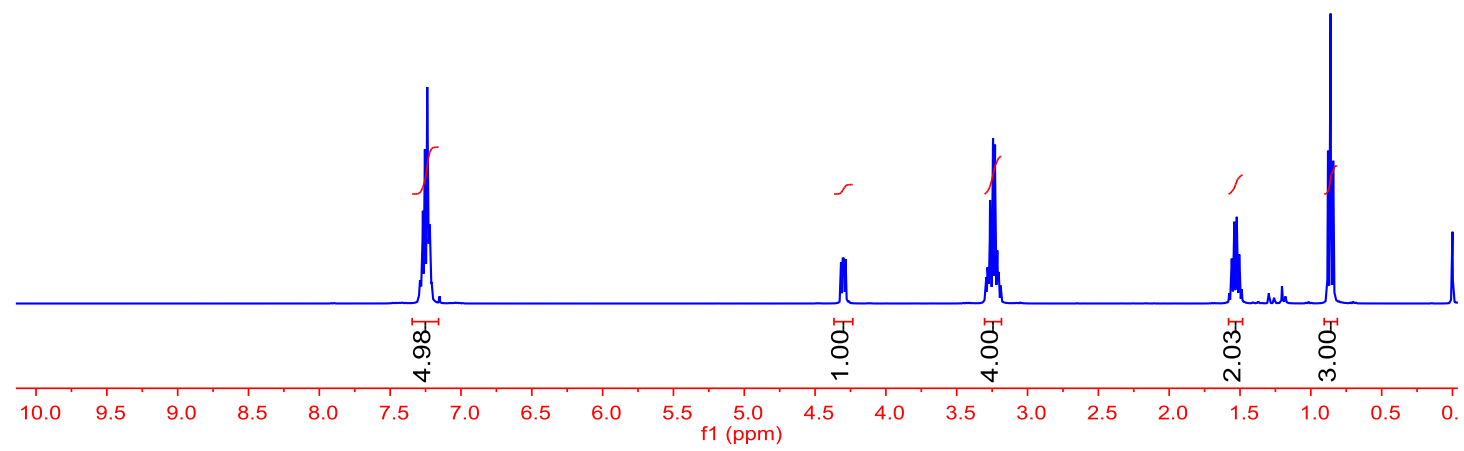

ํํㄹ N

$\begin{array}{ll}9 & m \\ 0 & 0 \\ 0 & 0\end{array}$

$\stackrel{\text { ஸे }}{\text { के⿻日禸 }}$

$\mathrm{O}^{n} \mathrm{Pr}$

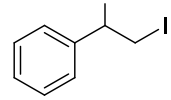

${ }^{13} \mathrm{C} \mathrm{NMR}\left(100 \mathrm{MHz}, \mathrm{CDCl}_{3}\right)$ of 9

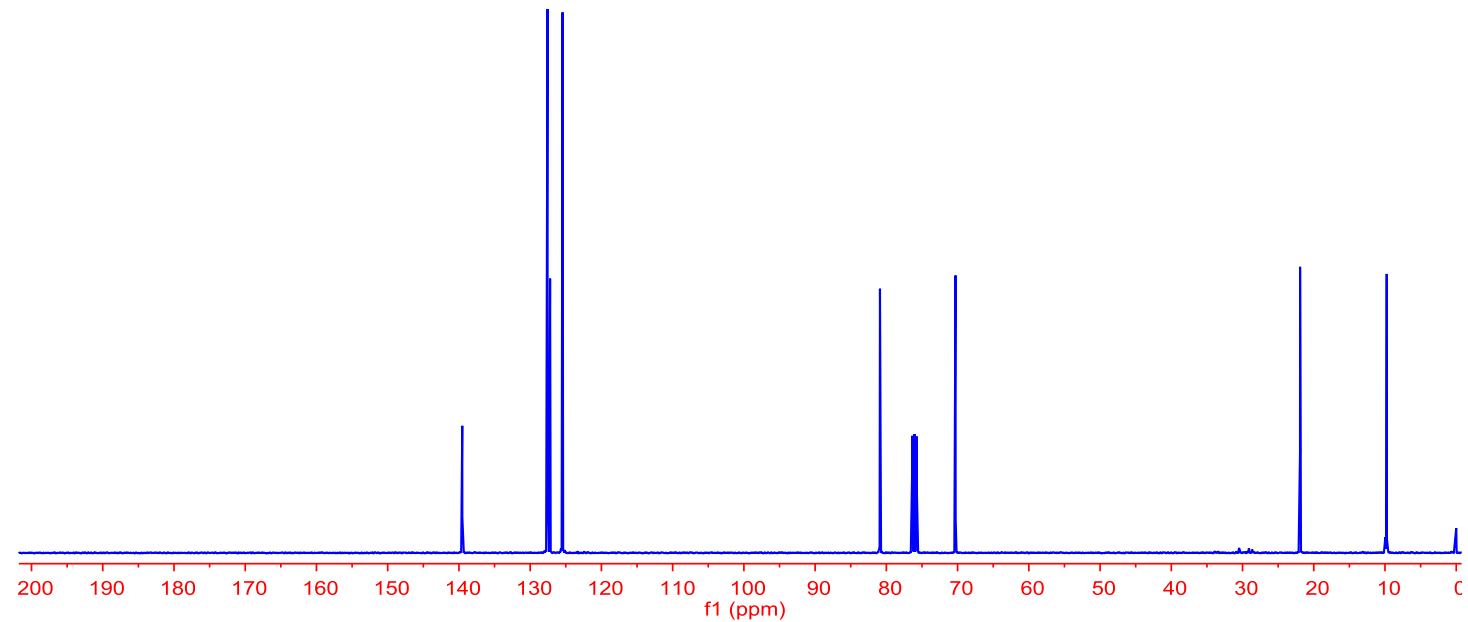




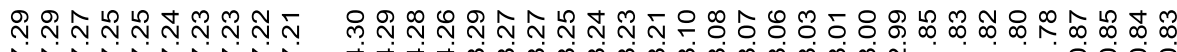

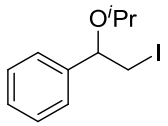

${ }^{1} \mathrm{H}$ NMR $\left(400 \mathrm{MHz}, \mathrm{CDCl}_{3}\right)$ of $\mathbf{9 j}$

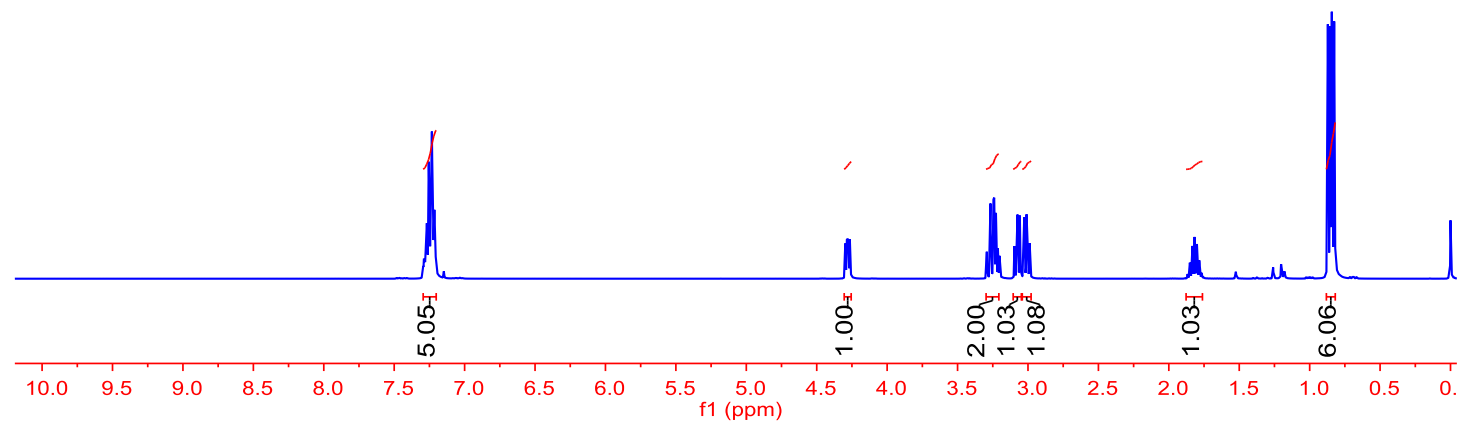

每

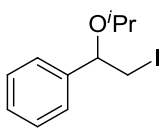

${ }^{13} \mathrm{C} \mathrm{NMR}\left(100 \mathrm{MHz}, \mathrm{CDCl}_{3}\right)$ of $\mathbf{9 j}$

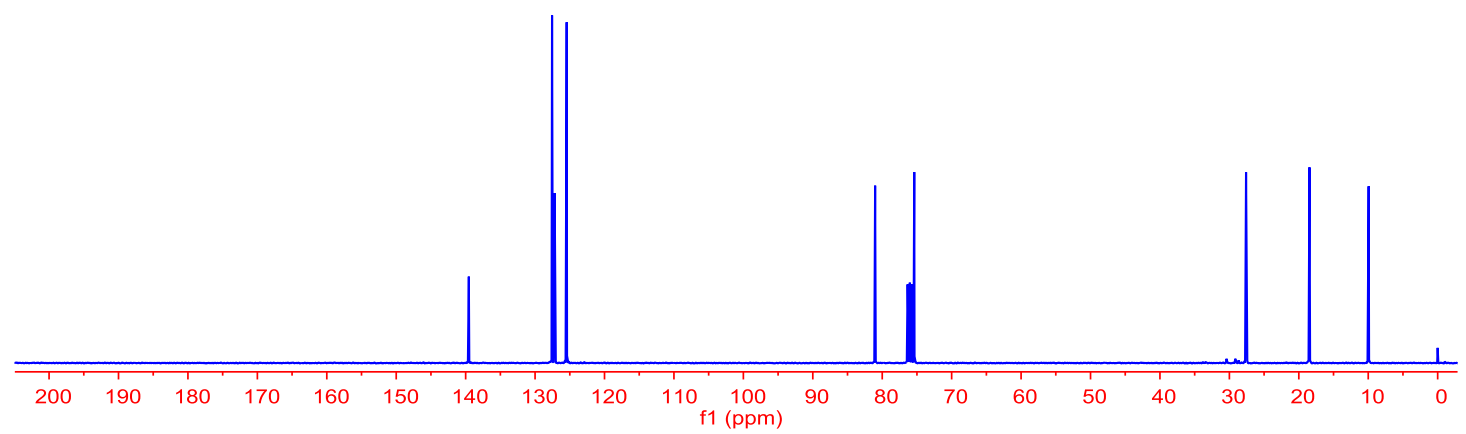




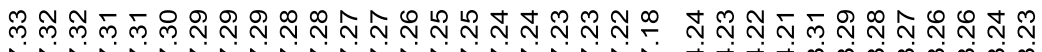

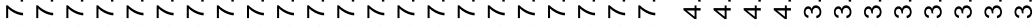<smiles>O=C(O)OC(CI)c1ccccc1</smiles>

${ }^{1} \mathrm{H}$ NMR (400 MHz, $\mathrm{CDCl}_{3}$ ) of $\mathbf{9 k}$
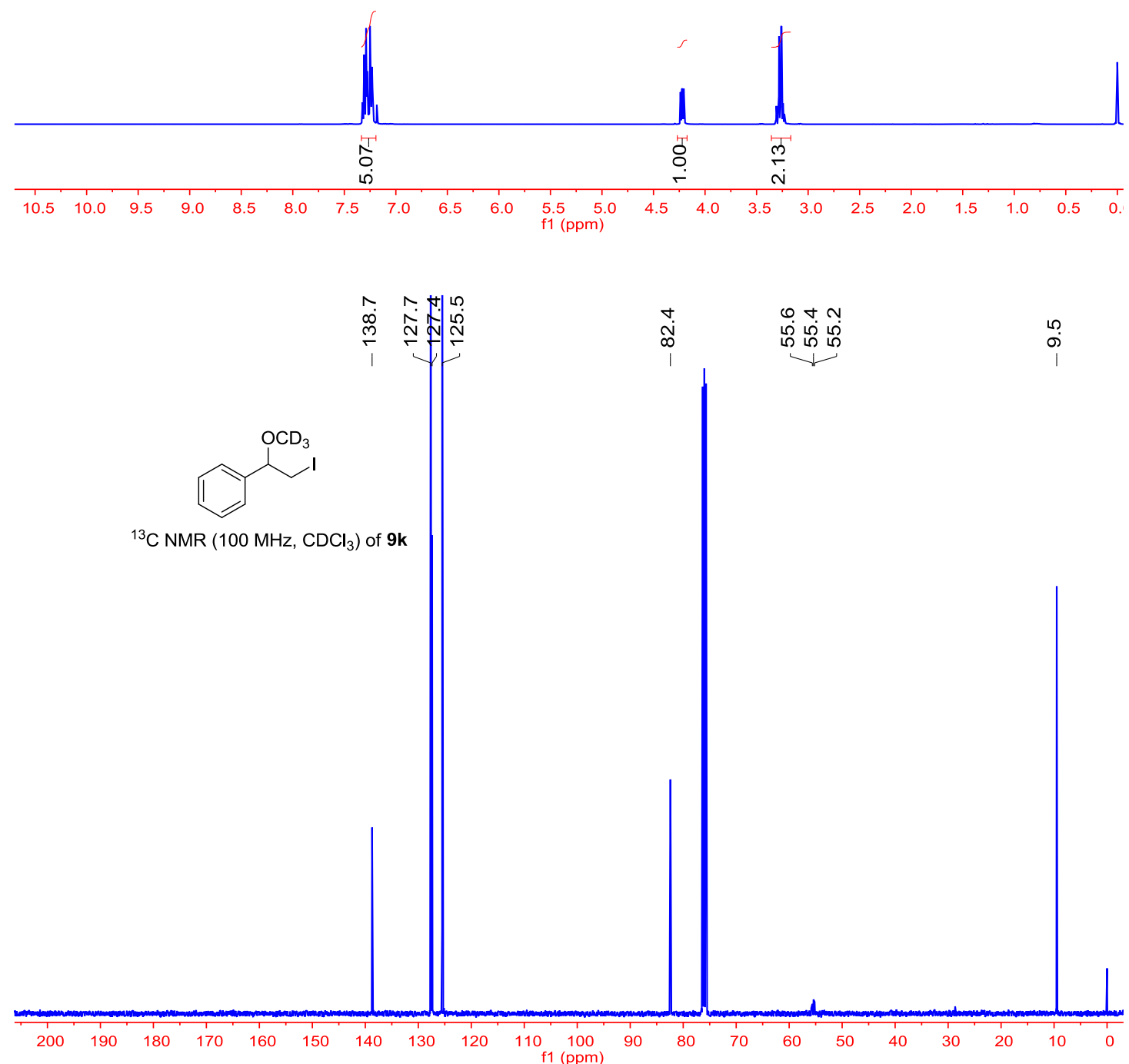


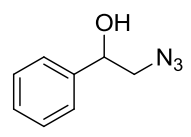

${ }^{1} \mathrm{H} \mathrm{NMR}\left(400 \mathrm{MHz}, \mathrm{CDCl}_{3}\right)$ of $10 \mathrm{a}$
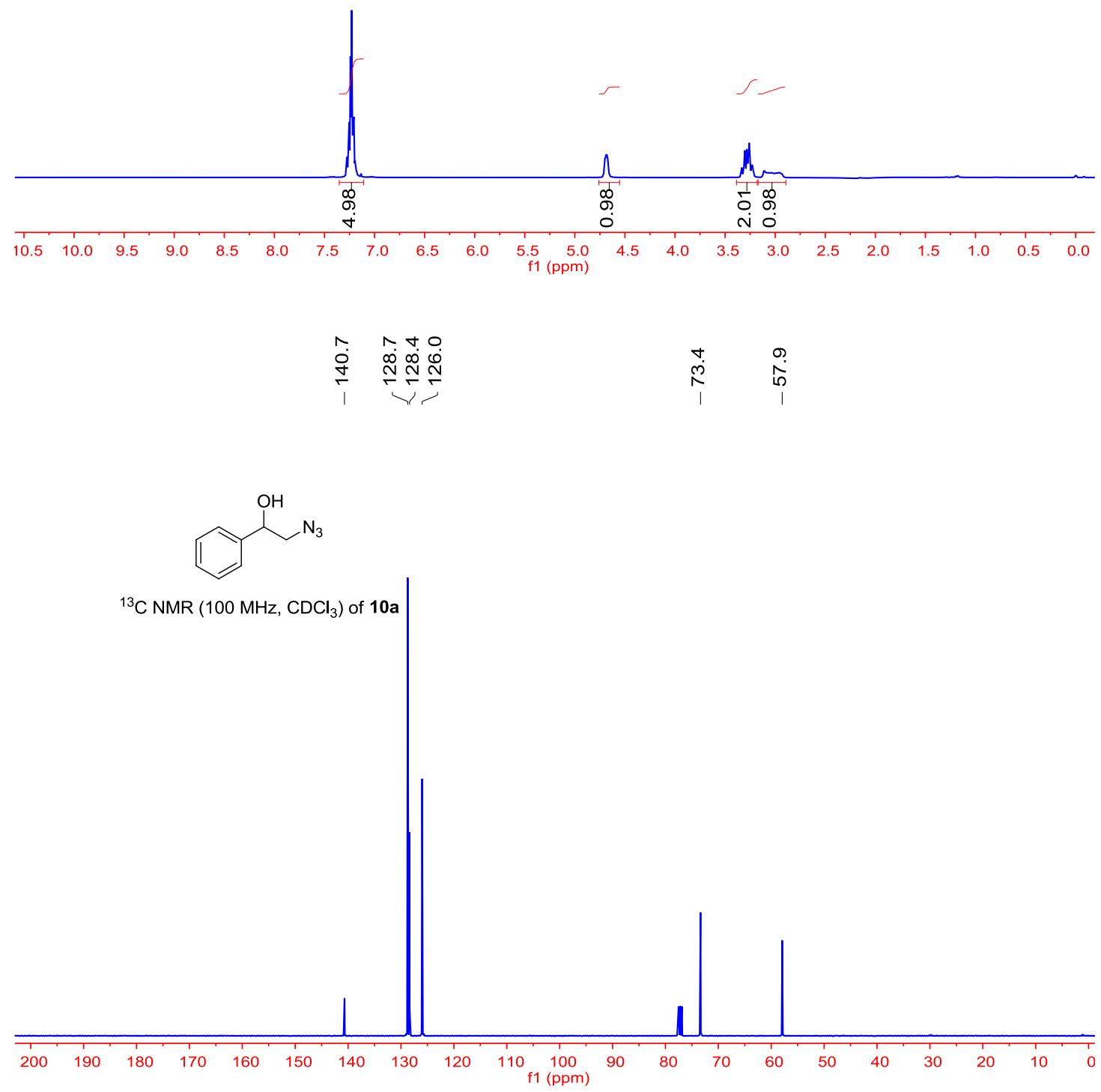


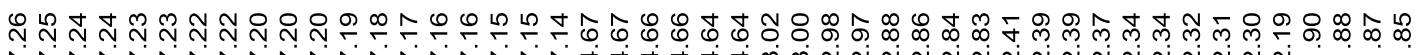

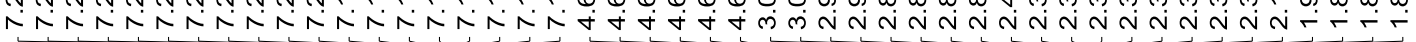

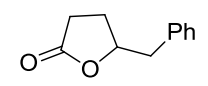

${ }^{1} \mathrm{H} \mathrm{NMR}\left(400 \mathrm{MHz}, \mathrm{CDCl}_{3}\right)$ of $\mathbf{1 0 b}$
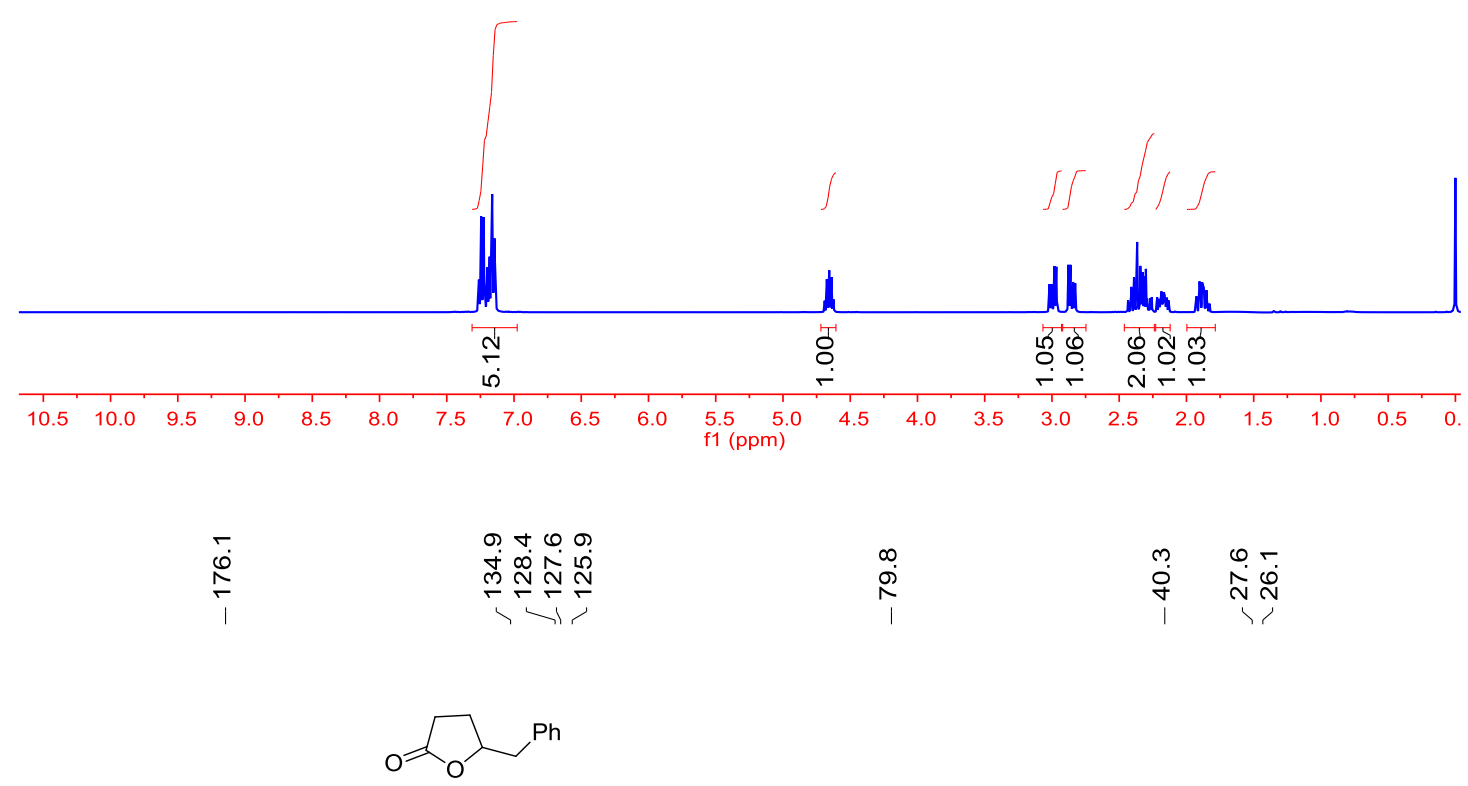

${ }^{13} \mathrm{C} \mathrm{NMR}\left(100 \mathrm{MHz}, \mathrm{CDCl}_{3}\right)$ of $\mathbf{1 0 b}$

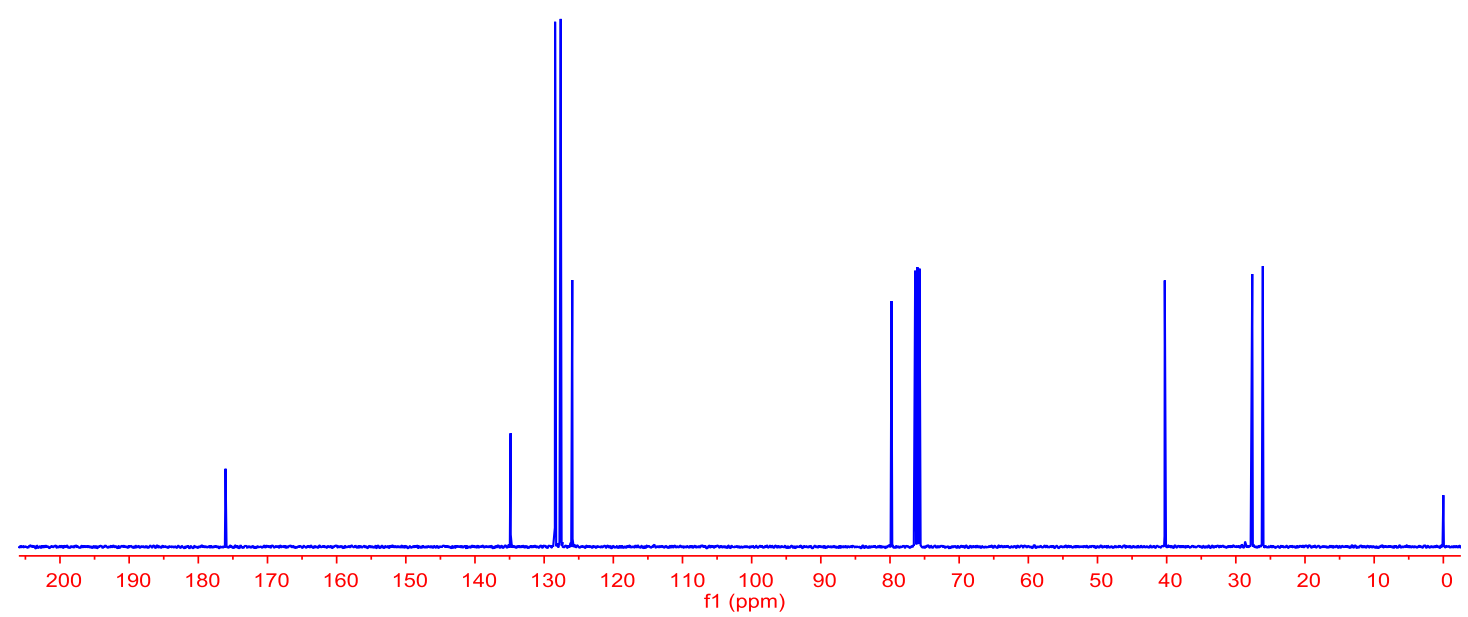

